\title{
Breaking through the clouds
}

Citation for published version (APA):

Thoonen, K. A. H. J. (2021). Breaking through the clouds: towards understanding sunburn, sun exposure and sun protection of children in the Netherlands. [Doctoral Thesis, Maastricht University]. Maastricht University. https://doi.org/10.26481/dis.20210429kt

Document status and date:

Published: 01/01/2021

DOI:

$10.26481 /$ dis.20210429kt

Document Version:

Publisher's PDF, also known as Version of record

\section{Please check the document version of this publication:}

- A submitted manuscript is the version of the article upon submission and before peer-review. There can be important differences between the submitted version and the official published version of record.

People interested in the research are advised to contact the author for the final version of the publication, or visit the DOI to the publisher's website.

- The final author version and the galley proof are versions of the publication after peer review.

- The final published version features the final layout of the paper including the volume, issue and page numbers.

Link to publication

\footnotetext{
General rights rights.

- You may freely distribute the URL identifying the publication in the public portal. please follow below link for the End User Agreement:

www.umlib.nl/taverne-license

Take down policy

If you believe that this document breaches copyright please contact us at:

repository@maastrichtuniversity.nl

providing details and we will investigate your claim.
}

Copyright and moral rights for the publications made accessible in the public portal are retained by the authors and/or other copyright owners and it is a condition of accessing publications that users recognise and abide by the legal requirements associated with these

- Users may download and print one copy of any publication from the public portal for the purpose of private study or research.

- You may not further distribute the material or use it for any profit-making activity or commercial gain

If the publication is distributed under the terms of Article $25 \mathrm{fa}$ of the Dutch Copyright Act, indicated by the "Taverne" license above, 


\section{Breaking through \\ the clouds}

Towards understanding

sunburn, sun exposure and sun protection

of children in the Netherlands

Karlijn Thoonen

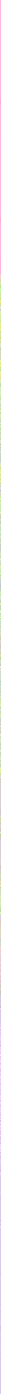





\section{Breaking through the clouds}

Towards understanding

sunburn, sun exposure and sun protection

of children in the Netherlands

There ain't a cloud in the sky or nothing

I see the birds, they fly high on something

I see a rainbow, purple and gold

But it's covered

Cause there's a cloud, she follows me around, wherever

But when these blue shade days are gone

I'm gonna stare into the sun' 
Cover: Stijn Kessels

Layout: Stijn Kessels

Printing: ProefschriftMaken

ISBN: 978-94-6423-189-2

๑ Karlijn Alexandria Henrieke Josephine Thoonen, 2021

The research presented in this thesis was conducted at CAPHRI Care and Public Health Research Institute, Department of Health Promotion, of Maastricht University. CAPHRI participates in the Netherlands School of Public Health and Care Research CaRe.

The studies presented in this thesis were conducted with financial support from the Dutch Cancer Society.

All rights reserved. No part of this publication may be reproduced, stored in a retrieval system or transmitted in any form or by any means, electronic, mechanical, photocopying, recording or otherwise, without prior permission of the author, or the copyright-owning journals for previous published chapters. 


\title{
Breaking through the clouds
}

\author{
Towards understanding \\ sunburn, sun exposure and sun protection \\ of children in the Netherlands
}

\begin{abstract}
Proefschrift
ter verkrijging van de graad van doctor aan de Universiteit Maastricht, op gezag van de Rector Magnificus, Prof. dr. Rianne M. Letschert, volgens het besluit van het College van Decanen, in het openbaar te verdedigen op donderdag 29 april 2021 om 16.00 uur door
\end{abstract}

\section{Karlijn Alexandria Henrieke Josephine Thoonen}

geboren op 28 januari 1991 te Elst 


\section{Promotor}

Prof. dr. H. de Vries

\section{Co-promotores}

Dr. L. van Osch

Dr. F. Schneider

\section{Assessment committee}

Prof. dr. S. Kremers (chair)

Prof. dr. B. Deforche (Ghent University)

Dr. J. Gubbels

Prof. dr. L. Lechner (Open University Heerlen)

Prof. dr. R. Ruiter 
Table of contents

\section{Chapter 1}

General introduction

\section{Chapter 2}

A qualitative exploration of parental perceptions regarding their children's sun exposure, sun protection and sunburn

\section{Chapter 3}

Identification of socio-cognitive determinants of parental sun protection behaviours to optimize intervention development

\section{Chapter 4}

Childhood sun safety at different ages: relations

between parental sun protection behaviour

towards their child and children's own sun

protection behaviour

\section{Chapter 5}

Children's sunburn exposed: Identification of sun exposure and parental sun protection patterns

\section{Chapter 6}

Are environmental interventions targeting skin cancer prevention among children and adolescents effective? A systematic review

\section{Chapter 7}

General discussion

Impact paragraph

References

Summary

Samenvatting

163

Curriculum Vitae

Publication list

Dankwoord 
chapter one

General introduction 
'If we wait for the moment when

everything, absolutely everything

is ready, we shall never begin.

— Ivan Turgenev 
This chapter describes the epidemiological background of skin cancer, opportunities for its (primary) prevention and the urgency for further research regarding skin cancer prevention. The importance of targeting parents and their children in this matter is explained. Moreover, the contemporary situation with regard to skin cancer - and its primary prevention - in the Netherlands will be clarified. The conducted studies for this thesis are formulated at the end of this chapter, after which an outline of this thesis is provided.

\section{Skin cancer explained}

Skin cancer includes non-melanoma (NMSC) and melanoma skin cancer, the latter also known as malignant melanoma. NMSC arises from keratinocyte cells which function as defence mechanism to protect the skin against ultraviolet radiation (UVR), heat or bacteria, whereas melanoma develops from melanocyte cells, involved in skin protection as well as pigmentation of the skin (1-3). NMSC includes basal-cell carcinoma (BCC) and squamous-cell carcinoma (SCC) (4). The anatomical distribution of NMSC is similar and most often detected on sun-exposed parts of the body such as the face, neck, ears, hands and - for BCC - the trunk (5). Melanoma can develop on any location of the body (6). Where BCC has a minimal potential of metastasis, approximately $4 \%$ of SCC and $20 \%$ of melanoma metastasize to other organs respectively $(7$, 8). Patients with NMSC, however, are at two- to threefold risk for developing melanoma (9). Besides skin malignancies, UVR can cause cataract $(10,11)$ and premature skin damage (12).

\section{Epidemiology}

Skin cancer is the most common type of cancer worldwide (13). Global incidence rates are highest for BCC, followed by SCC and melanoma (4) and all three types of malignancies are most common among fairskinned populations $(4,14)$. In the Netherlands, SCC rates are highest among men, whereas melanoma rates are highest among women (8). On a global level, skin cancer has increased dramatically in the last decades, with epidemiologic studies reporting doubled (15-17) to even tripled (18) incidence rates since the late 1980's. Recent estimates indicate that NMSC is the fifth most prevalent cancer among men and women (13), and that melanoma incidence rates have risen more rapidly than any other cancer type in the past 50 years (19). Melanoma is the most fatal and aggressive form of skin cancer with an estimated global mortality rate of nearly 60.000 in 2015 (20). Various reasons are underlying of the major increase in global skin cancer rates, such as ageing populations, changed clothing styles, improved diagnosis and increased exposure to UVR $(14,19,21)$.

\section{Burden of skin cancer}

In the Netherlands, NMSC rates have nearly quadrupled (11) and melanoma incidence nearly tripled in the last three decades (8). The annual age-standardized rate (ASR) for melanoma incidence in the Netherlands was 19.4 per 100.000 in 2012 (19), and 25.7 per 100.000 cases in 2018. As a comparison, Australia and New Zealand have the highest ASR globally (33.3 and 33.6) whereas in Europe, the Netherlands is among the countries with most alarming ASR, with only Denmark and Norway (27.6 and 29.6 respectively) exposing higher rates. Other western European countries such as the United Kingdom and France are depicting notably lower melanoma rates (15.0 and 13.6 respectively) (22). These numbers are likely to be an underestimation of the total skin cancer burden since 1) BCC is often not included in registration (23) and 2) only the first detected malignancies are registered while 30 to $60 \%$ of patients develop at least a second malignancy $(9,24)$. Melanoma incidence is predicted to rise even further $(8,25)$ and it is expected that all skin cancers combined will increase with $5-10 \%$ in the following years (11) in the Netherlands. 
Presently, the Netherlands are among the five countries globally with both highest relative melanoma incidence as well as mortality rates (20). In 2017, 796 people died from melanoma in the Netherlands (8) and in 2018, 60.712 deaths were attributable to melanoma on a global level (26). Melanoma death rates were signalling relative stability across countries, whereas age-standardized mortality rates in the Netherlands increased from 2.2 per 100.000 person-years in 1989 to 3.9 respectively in 2009 (27, 28). Currently, a relative stability in death rates is also indicated in the Netherlands (8), with men showing worse prognosis than women (1.37 excess relative death rate) (29). A recent study comparing melanoma mortality trends across 31 countries signals globally increased death rates among males, versus decreased or stabile death rates among females as well (30).

The burden of increased skin cancer incidence is further reflected in its treatment costs. Skin cancer treatment can exist of a combination of surgical tumour removal, radiotherapy, chemotherapy or immunotherapy and is dependent of the location, stage and progression of the tumour $(31,32)$. In 2015, health care costs were estimated at 4.8 billion dollars for NMSC and 3.3 billion dollars for melanoma in the United States (33), compared to 703 million for NMSC and 201 million for melanoma in Australia (34, 35). In the Netherlands, healthcare costs for SCC and melanoma amounted to nearly 169 million euros in 2017, accounting for $2.9 \%$ of the total treatment costs of newly diagnosed diseases (36). The total healthcare burden of skin cancer treatment, including BCC and cataract, was recently estimated to amount to over 400 million euros per year (11) and numbers revealed that skin cancer is among the four most costly cancers in the Netherlands (37). Moreover, the number of patients in need of skin cancer health care is predicted to increase with $35 \%$ in the next 10 years (8). Additionally, by predicting a significantly increasing dermatological workload, the need for a new skin cancer management strategy was proposed a decade ago (38). Recently, the urgency for optimizing skin cancer care, with a strong focusing on primary prevention efforts, was accentuated (39).

\section{Children as crucial target group}

Over twenty years ago, the International Agency for Research on Cancer (IARC) explicitly emphasized children's sun safety (40). The importance of sun protection during childhood is established in a large amount of studies. Intermittent sun exposure and sunburn acquired in early life particularly contribute to increased melanoma risk in adulthood (41-43). Moreover, received UVR-exposure in the first 18 years of life is associated with an increased risk for NMSC (44-46). Children are more susceptible for sun exposure since their skin produces lower levels of melanin, resulting in deeper infiltration of UV radiation into skin layers (47). A large number of children across countries regularly experience sunburn, with percentages ranging between 30 up to $60 \%$ per year (48-53). In the Netherlands specifically, approximately $20 \%$ of children have at least once experienced sunburn at (the primary) school (54), almost $30 \%$ in the foregoing summer season (55), $42 \%$ in the previous year (56) and over $77 \%$ throughout their lifes (55).

Research has indicated that performance of sun protection behaviours throughout childhood and adolescence can reduce lifetime risk of NMSC by $78 \%(57,58)$. Although precise percentages of reduced risk are not available for melanoma, reducing the amount of UVR-exposure and preventing skin damage in childhood is undoubtedly needed to eventually counteract the global melanoma epidemic $(42,59)$. Furthermore, from a developmental and psychological viewpoint, childhood is a crucial stage for the primary prevention of skin cancer, as health behaviours being established during early life are likely to persist into adulthood $(60,61)$. Thus, the effort that is made during early life provides both short-term health benefits in terms of children's skin being protected, as well as long-term perpetuation of their own acquired sun protection behaviours. 


\section{Primary prevention of skin cancer}

Risk factors for developing skin cancer are, among others, exposure to UVR, a history of sunburns and fair skin complexions (Fitzpatrick skin type 1 or 2) $(59,62,63)$. Additionally, intermittently received doses of UVR are particularly associated with BCC and melanoma whereas cumulative UVR across one's lifespan is related to SCC (64). Ozone layer depletion and an ageing population only partly explain the excessively increased skin cancer incidence in the Netherlands. Changes in sun-seeking behaviour are assumed to be one of the main reasons for rising incidence rates on a global level $(19,65)$, and in the Netherlands, although insight in behavioural patterns is lacking (11). Given the fact that UVR-induced damage is the major risk factor for developing skin cancer, it is considered as one of the most preventable malignancies $(66,67)$. Several precautions to decrease the amount of UVR-exposure and subsequent sunburn risk are advised for the general population and children specifically by cancer- and health organizations throughout the world (68-70). To eventually counteract the alarming skin cancer incidence, primary prevention of skin cancer in the Netherlands - and thus the sun exposure habits of children specifically - is in need of perpetuated improvement.

\section{Parental sun protection strategies}

The family setting is critically important in adopting preventative behaviours and establishing children's sun safety. Parental sun protection behaviours can include both direct (parent-for-child performance of behaviour) and indirect (facilitating a context in which a child can perform behaviours him- or herself) behaviours (71). Direct behaviours include seeking shade, limiting the amount of time outdoors, putting on UV-protective clothing and attributes and applying sunscreen. It is specifically recommended to avoid the sun between 10 AM and 4 PM, putting on long-sleeved t-shirts, long pants - and preferably of tightly woven fabric, wearing sunglasses and a hat and using and frequently reapplying sunscreen with a sun protection factor (SPF) of at least $30(70,72)$. Additionally, the Dutch Cancer Society recommends parents to avoid children's UVR-exposure between 12 and 3 PM, re-apply sunscreen every two hours and to use at least seven tablespoons of sunscreen for the whole body (73).

Execution of parental sun protection behaviours illustrates variation across studies. For example, studies reporting parent-for-child sun protection behaviours demonstrate low $(74,75)$, moderate $(76,77)$ to relatively fair (78) behavioural performance. With regard to children, high correlations between parental and children's behaviour, as well as parental and children's experienced sunburn are reported $(51,79,80)$. Although children show ability to perform sun protection behaviours themselves to some extent, evidence is limited. Furthermore, differentiation in the age from which children take responsibility for their own sun safety is not thoroughly investigated yet $(77,79,81)$. Considering the high prevalence of sunburn among children, there is notable room for improvement in adequate parent-for-child sun protection behaviours (82).

Since children are not able to take full responsibility of their own choices, decisions and behaviours, parents and caretakers play a vital role in children's own sun protection behaviours $(71,83)$. They are significantly involved in teaching their children the importance of health behaviours and in supporting them in the acquisition of skills to perform such behaviours. The family setting is therefore an important contributor to children's health-related beliefs and behaviours, with the parenting style being evidently related to children's own performance of health-related behaviours $(77,84)$. As illustrated in health behaviours like dietary behaviour, physical activity and oral hygiene, parents have shown to play an influential role (85-88). The function parents have in increasing sun safety among their children is likely to be equally important as well, although strong evidence regarding effectiveness of parental-focused interventions, is so far limited $(77,79,82)$. 


\section{Preventative behaviours and its challenges}

Enhancing sun protection for children is considered complex for various reasons. Firstly, sun safety requires a multitude of sun protection behaviours aimed at decreasing sunburn risk $(89,90)$. Therefore, estimating which behaviours are warranted in specific situations to protect children's skin can be demanding (71). Secondly, the extent to which sun protection strategies are needed depends on one's susceptibility for skin damage. For instance, individual characteristics such as children's skin type and genetic predisposition in terms of skin sensitivity or a high incidence of nevi influence melanoma risk and therefore the urgency for sun protection measures (91). Thirdly, situational characteristics such as the duration of activities, the UV-index and the purpose (e.g. swimming or cycling) of these activities ask for different sun protection measures $(70,78)$. Lastly, environmental characteristics play a vital role in the necessity for adequate sun protection behaviours. The strength of the UV index, a measure of UVR level reaching the earth's surface and indicative factor for necessary sun protection strategies $(92,93)$, depends on time of the day, latitude, altitude, weather (cloudiness), the season and reflection of surfaces (e.g. water or snow) $(70,94)$.

The complexity of sun safety is further increased by the fact that UVR-exposure has both proximal and distal health consequences. Although sunburn is a short-term and recognisable health effect, long-term consequences such as an ageing skin, increased nevi and skin cancer are less well-known among general populations (95-100), and among children $(101,102)$. Besides aversive effects, sun exposure is beneficial as well, with positive health- and appearance-related effects of sun exposure further adding to the complexity of skin cancer prevention. First, exposure to UVR generates vitamin D and melatonin and elevates positive mood (103). Since vitamin $D$ is vital for bone and muscle health, there is an ongoing debate whether avoiding sun exposure causes vitamin D deficiency $(104,105)$, specifically among children (106). Second, sun exposure induces the skin to produce melanin as a protective response, which results in tanned skin $(3,96)$. Having a tanned skin is often associated with a more beautiful appearance and cultural ideal in Western societies $(79,107,108)$ and among parents specifically $(51,75,89)$. Altogether, adoption of adequate sun protection behaviours is challenging since it originates through multiple pathways, involving many individual, social and environmental factors.

Besides high frequencies of reported sunburn across countries, the complexity of protecting children specifically is demonstrated when parental performance of sun protection behaviours is explored. The majority of studies report infrequent or suboptimal parent-for-child sun protection behaviours $(48,74,75)$, urging a need for improvement. Involving both direct (seeking shade, putting on clothing and applying sunscreen) and indirect (facilitating children to perform their own sun protection behaviours) parental sun protection behaviours in various sun exposure settings, is an underexposed topic across studies. Additionally, since distinct sun exposure patterns may relate to different skin cancer types (64), gaining insight in children's sun exposure and sun protection behaviours in different settings is vital. Studies investigating children's sun exposure, comprehensive parental protection behaviours, children's own behaviours and their relation with the major proxy for skin cancer - experiencing sunburn, are therefore needed.

\section{Understanding parental and children's sun protection behaviours}

Insight in both execution of comprehensive parental sun protection behaviours and establishment of these behaviours is warranted. One of the major aims in the field of health education and promotion, health communication and health psychology, is understanding why individuals change or not change their behaviours (109). Within the Intervention Mapping approach, understanding what determines a target behaviour is a crucial and preceding step in order to change these determinants. Knowledge of behavioural determinants guides the development and targeting of effective interventions (110). Behaviour change 
theories emphasize the importance of both individual and environmental determinants (110, 111), with socio-cognitive theories providing insight in intentional and deliberate establishment of behaviours (109), and dual-process models accentuating both direct and indirect influences of one's physical and social environment $(112,113)$.

So far, studies focusing on understanding parental determinants of sun protection are scarce, but those that did found high levels of knowledge among parents, yet misconceptions regarding sun protection (114, 115). High levels of knowledge often do not result in execution of parental sun protection behaviours $(116,117)$. Furthermore, although parents generally report positive attitudes regarding sun protection behaviours, they often feel difficulties with performing sun protection measures, which seemingly affects sun protection performance negatively (117-119). While the necessity for comprehensive sun protection measures is certain, a focus on solely sunscreen use is notable within the sparsity of literature $(77,79,120)$.

Insight in at-risk groups and environments is essential to target sun safety interventions and provide suggestions for tailored content (121). Given both the complexity of sun protection behaviours and individual risk factors, it may be important to eventually provide tailored messaging (e.g. regarding one's skin type, lifestyle, environmental characteristics) in interventions $(122,123)$. In order to increase the likelihood that desired parental sun protection behaviours are enhanced through interventions, it is first imperative to understand the complex parental sun protection behaviours by investigating the influence of both individual and environmental factors.

\section{Individual factors}

Social-cognitive models imply that individual behaviour is largely determined by cognitive determinants, stating behaviour originates deliberately (124-126). Some of these models have been tested, demonstrating the predictive value of socio-cognitive determinants (i.e. attitudes, social norms and self-efficacy) in individual sun protection intentions and behaviours (127-129). Yet, research on explaining parent-for-child sun protection behaviours is in a preliminary stage. Recent studies indicate the influential role of sociocognitive determinants in parental sun protection behaviours for very young (2-5 years) children, in which role-related beliefs and anticipated regret (130) and self-efficacy, action planning and habit (131) are associated with parent-for-child sun protection intentions and behaviours. In a Dutch context, previous research has demonstrated the predictive role of risk-perceptions (132) and action planning (133) for parental sunscreen use. Since the influence of social and cultural factors in establishing health behaviours is substantial $(134,135)$, more insight in socio-cognitive determinants underlying comprehensive parentfor-child sun protection behaviours in a Dutch context is warranted in order to develop evidence-based interventions.

\section{Environmental factors}

Since the core risk factor for skin cancer is exposure to UVR, the role of the physical environment is unmistakably important to consider when developing interventions. This is also illustrated by recommendations from the Surgeon General, which include the provision of shaded areas at public venues (6). From a social ecological perspective, behaviour originates through interactions between individuals and their social, physical or political environments (112). The encouraging role of intervening on these various levels of influence was already recognized for parents and children specifically three decades ago (136). In the early 80 's, Australia set the example with the national multi-component SunSmart strategy, by creating environments facilitating sun protection behaviours (137). Findings from three decades of SunSmart activities show encouraging results in which environmental efforts seem promising in enhancing sun protection behaviours $(138,139)$. For example, interventions in the political (e.g. mandatory hat use at primary schools (140)), or economic (e.g. reducing the price of sunscreen (141)) environment can promote sun protection behaviours. By adapting the physical environment and implementing cues such as accessibility of sun protection resources (e.g. shade and sunscreen availability, including the UV-index in weather 
forecasts), sun protection behaviours can be encouraged as well (138). An extensive review conducted in the United States found some indications that environmental adaptations promote sun protection behaviours among various target groups, although a lack of understanding about stand-alone effects and working mechanisms of these interventions was noticed (82). Although evidence is unavailable, findings indicate that environmental cues potentially trigger parental and children's shade-seeking behaviours $(142,143)$. Despite the encouraging effect of these environmental cues, additional research on shade use and its integration as part of extensive skin cancer prevention strategies is needed, as suggested by the authors of a recent review regarding effects of shaded areas on sun protection behaviours (144). Specific knowledge about effective health promoting interventions at various levels of environmental influence on parental as well as children's sun protection behaviours is not available yet.

\section{Aims of this thesis}

Although several efforts have been devoted to raising awareness about the importance of skin cancer prevention, both scientific insights into, as well as practical implications for enhancing sun protection behaviours are warranted. To successfully develop, implement and evaluate sun safety interventions for parents and their children, systematically planning health promoting interventions is essential $(109,145)$. The studies described in this thesis therefore aim to understand and explain children's sunburn, sun exposure and parental sun protection behaviours and evaluate available health promotion interventions. Data from a longitudinal cohort study with four measurements was used for three Chapters in this thesis. The ultimate goals of this thesis were threefold:

\section{Exploring parental socio-cognitive determinants and perceptions}

As previously stated, insight in the onset of parental sun protection behaviours is currently lacking in the Netherlands. Therefore, this thesis aimed to investigate parental socio-cognitive determinants of various direct (i.e. applying sunscreen, wearing clothing, seeking shade), and indirect (supporting children to perform protective measures themselves) sun protection behaviours. A comprehensive qualitative study among parents was conducted in order to explore socio-cognitive determinants for all sun protection behaviours in several sun exposure situations, together with parental perceptions regarding sunburn situations. The findings of this study are described in Chapter 2. In Chapter 3, quantitative determinant analyses investigating the relevance of extensive parental socio-cognitive determinants in predicting comprehensive parental sun protection intentions and behaviours, are outlined.

\section{Unravelling sun protection behaviours}

Exploring parent-for-child sun protection behaviours, together with the extent to which children take control over their own sun safety, is essential. Current insight in behavioural patterns of comprehensive sun protection behaviours among both parents and their children is lacking in the Netherlands. Therefore, execution of parental sun protection behaviours in relation with children's own sun protection strategies was examined. Children's age and sex were considered to investigate the age from which children are able to perform sun protection behaviours themselves and explore differences between boys and girls. These findings are outlined in Chapter 4. Further, as the complex relationship between parental sun protection behaviours and sunburn among children is unclear, Chapter 5 describes children's sunburn and its association with their acquired sun exposure and sun protection behaviours in order to unravel sunburn occurrence and identify children at-risk. 


\section{Investigating available health promoting interventions}

As environmental factors on children's sun safety also play a significant role, an overview of current evidence regarding the effectiveness of health-promoting environmental interventions targeting children and adolescents is described in Chapter 6.

The studies outlined in this thesis are the first to investigate children's sunburn, UVR-exposure and performance of sun protection behaviours in the Netherlands and therefore help to gain insight in current protection levels and at-risk groups. This thesis furthermore provides input for the development and targeting of sun safety interventions for parents and their children, needed to address the ongoing skin cancer epidemic in the Netherlands. 
chapter two

\section{A qualitative exploration of parental perceptions regarding their children's sun exposure, sun protection and sunburn}


'Do the best you can until you know better.

Then when you know better, do better.'

— Maya Angelou 


\section{Background}

Sun protection among children is of utmost importance since sunburn in early life is a major risk factor for skin cancer development. Because parents play a vital role in enhancing sun safety among children, this study explored parental perceptions concerning sun exposure, sun protection behaviours, and sunburn in children. Additionally, the context in which children experience sunburn in order to assist the development, optimization, and targeting of sun safety interventions for parents is revealed.

\section{Methods}

A qualitative study design, using a semi-structured interview guide addressing several themes (e.g. sun exposure, sun protection, and sunburn experiences), was used. Data were collected in the Netherlands in the fall of 2019. Parents were recruited via purposive sampling at schools, youth services centers, and social media. Execution, transcription, and coding of the interviews was done by two researchers, using the qualitative analyzing program Nvivo (interrater reliability of $d=.84$ ).

\section{Results}

In total, 26 interviews were performed ( $n=17$ mothers, $n=17$ daughters, aged between 4 - 11 years). Parental perceptions and recall of their child's lifetime sunburn were frequent, even though all parents reported using at least one sun protection measure during sun exposure situations. Parents were often unaware of their child's sunburn. Regarding sun protection measures, parents demonstrated an overreliance on sunscreen, often failing to adequately protect their children's skin. Water-related activities, a lack of shade, and lack of knowledge regarding UV-index were often related to sunburn. Moreover, unexpected sun exposure or longer exposure duration than initially planned were reported as challenging situations. The majority of parents had positive perceptions regarding tanned skin for both themselves as for children.

\section{Discussion}

This study provides directions for future skin cancer prevention efforts targeted at both parents and their children. Since a lack of knowledge regarding sufficient sun protection measures and sunburn occurrence in various situations was reported, educational efforts are warranted. Additionally, focusing on clothing, shade-seeking, and adequate sunscreen use is recommended to increase children's sun safety. By intervening in the physical environment as well (e.g. providing shady areas), sun protection barriers can be reduced. Lastly, the general positive attitude toward tanned skin evident in this study is certainly worthy of attention in future interventions. 


\section{Introduction}

Melanoma and non-melanoma skin cancers currently represent the most common types of cancer among fair-skinned populations, with exceedingly increasing incidence rates worldwide in recent decades $(4,14$, 146). The lifetime risk of developing melanoma, the most fatal form of skin cancers, was estimated at 1 in 39 for men and 1 in 58 for women in the United States (147) compared to 1 in 16 and 1 in 24 respectively in Australia (148). In Europe, the highest lifetime risk is observed in Nordic countries, ranging from $1.3 \%$ to $1.6 \%$ (149). Despite the relative stability of melanoma mortality rates across the United States, Australia, and Europe (14), management of skin cancers places a considerable and expanding burden on healthcare systems, and this is expected to worsen as skin cancer incidence increases (150). In the United States for example, the average yearly costs for the treatment of skin cancers rose by $126.2 \%$ between 2002 and 2011 (33). In Belgium, the economic burden of skin cancer was recently forecast to triple in the next two decades (151). Exposure to UVR (Ultraviolet radiation) and sunburn are considered major risk factors associated with melanoma development (4), where UVR-exposure and a history of one or more cases of sunburn during childhood are particularly harmful $(41,42,62)$.

Although incidence rates have risen excessively in the past decades, skin cancers are considered to be one of the most preventable malignancies $(6,152)$. By protecting the skin and limiting the amount of unprotected exposure to UVR, skin cancer risk can be decreased. Various sun protection behaviours, such as avoiding the sun during peak UV-hours, wearing protective clothing, and applying a broad-spectrum sunscreen with SPF 15 or higher, are recommended strategies. Additionally, simultaneously practicing multiple sun protection methods is considered essential for adequate UVR-exposure protection, which accentuates the importance of primary prevention efforts $(6,69)$.

Despite the finding that sunburn incidence during early life profoundly increases the risk of melanoma (153), sunburn occurrence among children is highly prevalent, with recent percentages of children having experienced sunburn at least once in the previous year ranging from $28 \%$ to $60 \%(48,49,154)$. Besides the urgency to prevent sunburn during early childhood, establishing sun protection behaviours among children is especially advantageous since formation of health behaviour patterns takes place in this critical period and such behavioural patterns are likely to persist into adulthood $(71,155)$. Parents and caregivers function as influential role models in the acquirement of children's own sun safety behaviours $(51,77,79)$, which is notable throughout childhood. Young children generally depend on the direct protection behaviours that their parents apply $(79,82)$, whereas older children can indirectly learn to perform sun protection behaviours themselves through facilitation from their parents $(156,157)$. Although parents are recognized as crucial agents in teaching children health behaviours, a minority of sun safety interventions are directed at parents and those that are have limited effects on parental sun protection practices $(82,158,159)$. Acquiring adequate sun safety practices among both children and their parents is therefore considered essential if future skin cancer rates are to decrease.

Although recommended strategies to increase sun safety are clear, inadequate sun protection during childhood is common $(48,74,75)$. Suboptimal sun protection among children could be explained by weak intentions to execute sun protection behaviours. For example, poor intentions can be caused by low levels of knowledge (e.g. not knowing what sun protection measure is needed), attitude (e.g. not believing in the importance of sun protection), or self-efficacy (e.g. not feeling able to perform sun protection behaviours) $(127,131)$. Inadequate knowledge concerning the UV index and the necessity of sun protection measures (114, $115,160,161)$, or experienced difficulty with performing sun protection behaviours $(55,117)$, can negatively affect behavioural outcomes. Moreover, studies have shown that parents' positive attitudes toward tanning can result in inadequate sun protection behaviours as well $(75,161)$. However, insufficient sun protection could also be explained by intentions not being translated into actual behavioural performance (128). For instance, perceived barriers or a lack of skills or action plans to perform sun protection can hinder sun protection intentions resulting in behaviour (162). Barriers such as children's refusal of sun protection (118) or sun protection methods being perceived as impractical or unpleasant $(53,163)$ negatively affect sun protection behaviours. This intention-behaviour gap has been previously demonstrated in studies in which 
parental intentions to apply sun safety measures did not result in actual sun protection behaviours (131, 133). Although intentions play a decisive role in establishing behaviour change, it is important to recognize that not all behaviour originates as a result of a reasoned approach but can also be non-deliberate and automatically generated by environmental cues (164) Several characteristics in the physical environment can influence whether or not behaviour is performed (i.e. physical, economic, sociocultural, and economic $(112,165)$ ). Moreover, as the Environmental Research Framework for Weight Gain prevention (EnRG) states, different environmental levels of influence are distinguished as well, such as micro- (i.e. family, school) and macro- (government) settings (165). With regard to sun safety interventions, adaptations in the physical environment (e.g. provision of shady areas) can trigger sun protection behaviour $(166,167)$. Especially since the dosage of received UVR by children is highly dependent on characteristics in the physical environment $(142,168,169)$, insight into the environmental context affecting parental sun protection behaviours is needed.

Specific insight into situations in which children's sun safety is challenging and the risk of sunburn is elevated is lacking. Additionally, insight into contextual and situational factors besides motivational factors burdening children's sun safety is warranted to understand sunburn occurrence more clearly (170). Therefore, comprehending facilitating factors and barriers causing insufficient sun protection practices among parents in detail is needed.

In conclusion, insight into situational as well as environmental barriers hindering parental sun protection behaviour needs elaboration. Moreover, in order to understand the challenges related to children's sun safety in view of those factors more clearly, exploration of parental perceptions regarding sun exposure and protection behaviours is necessary. This study therefore aims to unravel parental perceptions regarding their children's sun exposure, sun protection, and sunburn, in order to assist the development, optimization, and targeting of children's sun safety interventions directed at parents.

\section{Methods}

A qualitative study design with semi-structured individual interviews was used, which enabled various themes to be addressed and a broad input from participants (171). Data collection took place between September and November 2019. The interviews were conducted individually and by telephone, since anonymity was considered highly important (172). Moreover, this approach enabled participants to remain in their private home setting, which was regarded most suitable given the possible sensitivity of some questions. Participants, and in particular parents, may be unwilling to acknowledge behaviours or circumstances that deviate from societal norms (173), especially when a child's well-being is under discussion, and parents can experience guilt or feel they are being blamed (174). Ethical approval for this study was granted by the Research and Ethics committee of the Faculty of Health, Medicine and Life Sciences of Maastricht University, as covered by the general license of the Master Health Education and Promotion. Verbal informed consent of all parents was obtained and recorded. This study is reported in accordance with the COREQ checklist for qualitative research (175).

\section{Participants and recruitment}

Parents with at least one child aged between 4 and 12 and speaking either Dutch or English were eligible for inclusion in this study. A flyer was created in which parents were requested to participate in an interview to share information about their children's sun exposure during the previous summer season. The flyer was disseminated via several recruitment channels; primary schools and a childcare centre were approached, after which social media sites were used. These channels were carefully chosen since they were expected to recruit information-rich cases for this study (176). Firstly, three primary school boards in the east of the Netherlands, and one large day-care centre in the south were approached by e-mail, explaining the purpose of the study and asking them to distribute an online recruitment flyer. Secondly, social media platforms were used for further recruitment. Facebook was used to gain access to various pages and groups specifically 
directed at parents (e.g. pages containing education and information about parenting, health centre pages for children and families, and youth healthcare organizations). Permission for recruiting participants by posting an online flyer was requested by directly contacting the group moderators online. See Figure 1 for a flowchart of the recruitment process.

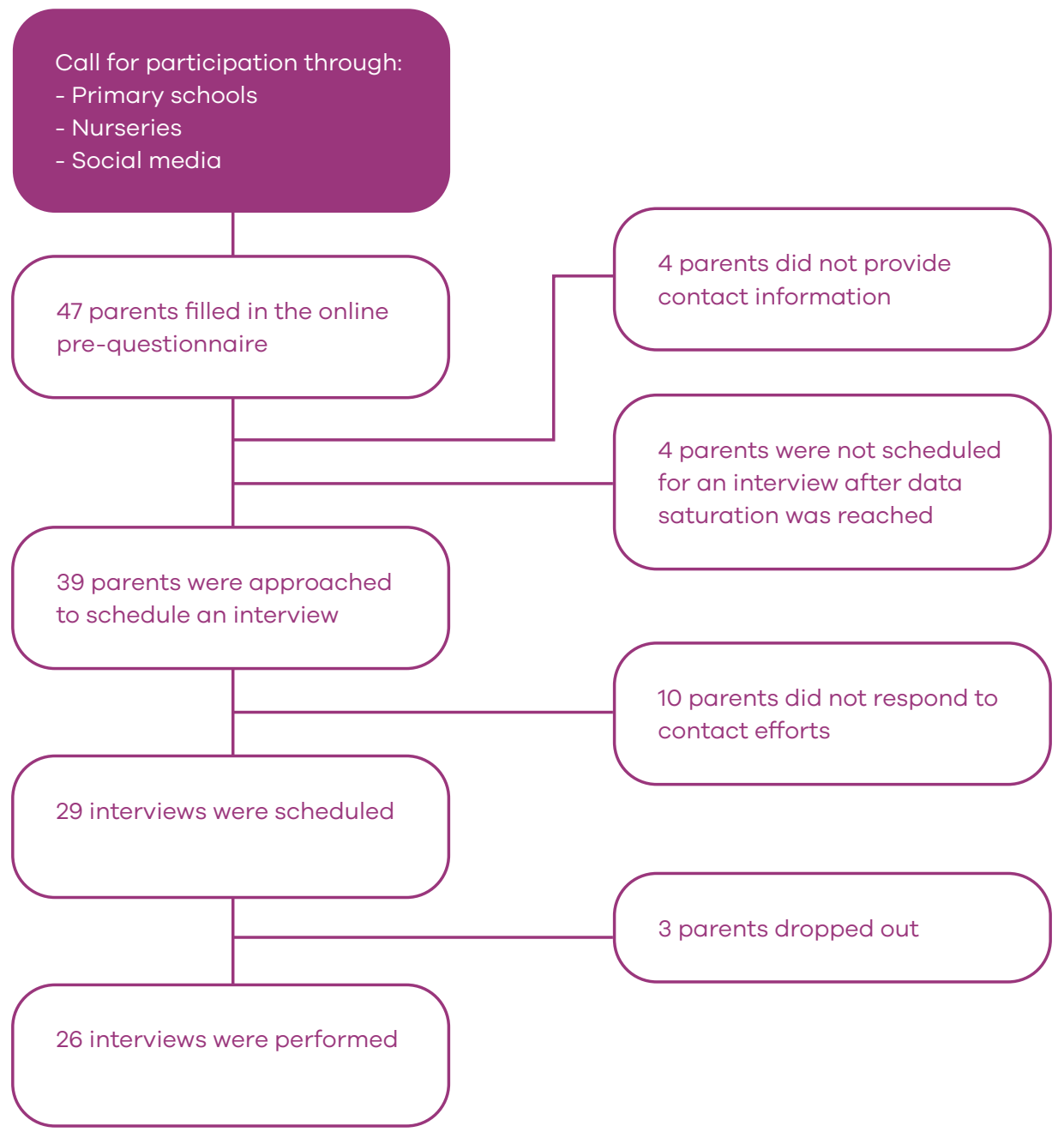

Figure 1. Flowchart of participants' recruitment 


\section{Procedure}

Parents could indicate their interest in participating in this study by clicking on a link provided in the recruitment flyer, after which they were directed to a survey website (Qualtrics, Provo, UT (177)). The website specified the aim of the study, including gaining information concerning children's sun exposure and sun protection during the previous summer. Research members' names and professions were also mentioned. On the website, parents were asked to fill in the age of their children as a check for eligibility criteria. Other demographic questions (i.e. parental sex, educational level, children's sex) were also assessed. Finally, parents were asked to indicate whether they were willing to allow the researchers to approach them via telephone and e-mail to schedule a telephonic interview by providing their contact information. The researchers selected parents using a purposive sampling strategy (178), striving for an equal distribution of demographic characteristics of both parents and their children since differences in terms of demographic variables can affect health-related outcomes (179). An equal as possible distribution of children's ages was the primary goal and a representativeness of children's sex the secondary goal when approaching interested parents for the interviews. With regard to children's age, a representation of children from all three phases of the Dutch primary school system was strived for ( 4 to 6 years, 7 to 9 years and $>10$ year). Children's skin type was unknown before scheduling the interviews. Aside from the demographic characteristics, the researchers were not familiar nor affiliated with the participants in any way. When approaching the parents, further information regarding the study aim was provided and telephonic interviews were scheduled. Parents then received a confirmation e-mail along with a letter containing information about data collection procedures, recordings of the interviews, storage of data, participant's protection of anonymity, and their rights to withdraw from the study. After completing the interviews, participants received an incentive of a 10-euro gift voucher, which was sent to their home address. To increase reliability and validity, member checking was done by paraphrasing and summarizing the information provided throughout the interviews (180). Participants did not receive the verbatim transcripts. After concluding the study, an overview of study results, accompanied by an infographic including sun protection information and tips, was sent to all participants. Both researchers had a professional background in patient communication in a healthcare setting. A semi-structured interview guide was used to structure the conversations. To accomplish both investigator and method triangulation, the interviews were alternately conducted by one of the two researchers, while the other researcher present generated interview notes based on observations (181).

Before commencing the interviews, audiotape devices were switched on and the interviewer asked whether participants had read the study information letter and whether they had any questions regarding the information. If participants had not read the information, the interviewer outlined the most important study aspects as mentioned above and asked whether participants had any questions. The interviewer then read out loud a prepared text in which the study purpose, data collection and data storage methods, and participant's rights from the previously sent information letter were summarized, and verbal informed consent was obtained. Thereafter, parents were asked to answer all questions for one specific child. Before the interview continued, the researcher explained the purpose of the interview and told participants that they were being asked about their personal experience regarding all the themes, and emphasized that there were no right or wrong answers. Each interview lasted approximately 30 minutes. To increase reliability and validity, the participant's information was summarized and paraphrased regularly throughout the interviews (180). As well as interview guides, a field note format was used in which the overall impression of the conversation as well as practical issues, critical reflections of the researcher's performance, and possible biases were observed and reported (182). Interviews were conducted until sufficient information was obtained and data saturation was reached (183), enabling the researchers to proceed with the data analysis process.

\section{Interview guide}

Prior to the interviews, a semi-structured interview guide was developed, consisting of three main themes, namely, perceptions regarding: (1) sun exposure; (2) sun protection behaviours; and (3) sunburns. Additionally, parental and children's skin type were assessed and estimations of children's skin type were 
later formulated by the researchers based on the Fitzpatrick skin type classification (184). Existing literature concerning sun protection behaviours as well as concepts from the environmental EnRG framework (165) were used to frame the three themes. The interview guide was reviewed by the whole research team on language use and content, and was adjusted where necessary. To test the suitability and feasibility of the interview guide, three interviews were piloted. The research team discussed the preliminary results of the interviews before making slight adaptations to the questions and their sequence.

The first two themes of the interview guide consisted of questions regarding negative and positive aspects of sun exposure. These parts were preceded by an open-ended question about perceptions that parents held regarding the sun, after which prompting questions were asked to retrieve in-depth information about three domains, i.e. (1) health-related; (2) well-being (mental health); and (3) appearance-related. The third part of the interview guide entailed questions regarding direct parental sun protection behaviours, children's own sun protection behaviours as well as indirect parental protection behaviours (i.e. facilitating their child). This part was introduced by explaining that different measures can be taken to protect one's skin against the sun. In contrast to the previous parts of the interview, the questions regarding sun protection behaviours were more structured.

When participants were not very forthcoming, the researcher included prompt questions regarding the three main domains (171). In the first part, independent of whether participants mentioned it themselves, the researcher mentioned sunburn occurrence as a possible negative aspect of the sun. Then, the researcher asked whether the child had experienced sunburn in the previous summer season, after which the researcher asked detailed questions about this specific situation. If parents did not report a previous case of sunburn, the researcher asked them whether they could recall details from an earlier sunburn situation in their child's life. When parents stated that no sunburn had ever occurred, parents were asked to imagine a potential sunburn situation. Further in-depth questions regarding the characteristics of experienced or imagined sunburn situations were then posed. In the second part, again independent of participants mentioning it themselves, a tanned skin was introduced. Thereafter, in-depth questions were asked concerning parental perceptions as well as other people's opinions regarding children's tanned skin. The themes from the interview guide with exemplary items are depicted in Table 1.

\section{Table 1. Interview themes and exemplary items}

\begin{tabular}{|l|l|l|}
\hline Theme & Category & Exemplary items \\
\hline Negative aspects of the sun & Open associations & $\begin{array}{l}\text { 'What comes to mind when you think about } \\
\text { disadvantages of being in the sun?' }\end{array}$ \\
\hline & Prompted associations & $\begin{array}{l}\text { 'What possible disadvantages of the sun are } \\
\text { there regarding emotions or how you feel?' }\end{array}$ \\
\hline & Sunburn situation & $\begin{array}{l}\text { 'What was the purpose of the activity?' } \\
\text { 'Was there a lot of cloud coverage?' } \\
\text { 'How severe was the sunburn?' } \\
\text { 'What made sun protection in this situation } \\
\text { particularly difficult?' }\end{array}$ \\
\hline Positive aspects of the sun & Open associations & $\begin{array}{l}\text { 'What comes to mind when you think about } \\
\text { benefits of being in the sun?' }\end{array}$ \\
\hline & Prompted associations & $\begin{array}{l}\text { 'What possible benefits of the sun are there } \\
\text { regarding your health?' }\end{array}$ \\
\hline
\end{tabular}




\begin{tabular}{|l|l|l|}
\hline & A tanned skin & $\begin{array}{l}\text { 'How important is a tanned skin for you? } \\
\text { And how important is a tanned skin for you } \\
\text { when it concerns your child?' } \\
\text { 'How do you feel about your child having a } \\
\text { tanned skin?' } \\
\text { 'What do you think other people around you } \\
\text { feel about your child having a tanned skin?' }\end{array}$ \\
\hline Sun protection behaviour & Parental behaviours & $\begin{array}{l}\text { Direct: 'Which sun protection measures do } \\
\text { you apply when your child is exposed to the } \\
\text { sun?' } \\
\text { Indirect: 'To what extent do you support your } \\
\text { child in executing sun protection behaviours } \\
\text { him- or herself?' }\end{array}$ \\
\hline Children's behaviours & $\begin{array}{l}\text { 'Which sun protection methods does your } \\
\text { child apply him- or herself? } \\
\text { 'What would facilitate you or your child to } \\
\text { perform sun protection measures in the } \\
\text { future?' }\end{array}$ \\
\hline
\end{tabular}

\section{Data analysis}

After recording the interviews, verbatim transcripts were generated and imputed in the qualitative analysing program NVivo, version 12 (185). In accordance with the six phases of thematic analysis (186), first, each researcher individually open-coded one different and randomly selected transcript and developed a codebook. Then, the two codebooks were compared, and a new codebook was composed. The researchers then used this codebook during their independent coding of the same selection of five transcripts. Hereafter, an interrater reliability was calculated in NVivo, based on Cohen's Kappa, resulting in a high level of agreement $(\mathrm{k}=.84)$ between the two coding results $(187,188)$. Lastly, an adjusted version of the codebook was generated by mutual agreement after discussing small disparities by comparing coded passages. The finalized codebook was used to code the remaining transcripts and to formulate main themes and categories to enable the results to be interpreted. A third researcher (FS) observed and reflected on the analysing process to enhance objectivity. Thematic coding took place by clustering codes together in categories, followed by interpretation of these categories and their integration into several main themes. Again, discrepancies were discussed by comparing passages and mutually adjusting the categories.

\section{Results}

\section{Sample characteristics}

Forty-seven participants filled out the online questionnaire, of which forty-three parents submitted their contact information for scheduling an appointment. Thirty-nine parents were approached throughout the study period and an interview was scheduled with twenty-nine parents, based on a previously made selection according to children's and parental age, children's and parental sex, and educational level. Four interested parents were not scheduled for an interview after data saturation was reached. Three parents dropped out before the interview took place, due to being inaccessible $(n=2$, mothers) and health-related problems $(n=1$, father). The dropped-out parents all had a son (aged 6, 9 and 10). In total, twenty-six telephonic interviews with parents ( 17 mothers, 9 fathers) of children ( 17 daughters, 9 sons) aged 4 to 11 years $(M=7$ years, Modi $=4$, 
5 and 7 years) were conducted. The age of the parents ranged from 30 to 50 years $(M=40$ years, Modus $=44)$ and the majority of the parents were highly educated $(n=20 ; 76,9 \%)$. Most children had a very light $(n=6)$ or light $(n=8)$ skin type, and one child had a darker skin type based on the Fitzpatrick skin type classification. Almost all parents $(\mathrm{N}=25 ; 96 \%)$ reported that their child had experienced sunburn at least once in their lifetime. An overview of emerged themes and sub-codes is attached as a Supplementary file.

\section{Perceptions regarding sun exposure}

\subsection{Negative and positive perceptions}

Parents did not immediately mention general negative perceptions regarding sun exposure. Consequently, pro-active prompts by the interviewers regarding disadvantages were necessary. After prompting, negative aspects of the sun in general that were subsequently mentioned were most frequently healthand appearance-related. The risk of sunburn and of developing skin cancer was mentioned most often: "... Yes, too much UV-radiation, you read it everywhere nowadays, that, that it can cause skin cancer, ehm, yes, that is obviously the greatest disadvantage of UV-radiation". Additionally, damaged skin, dehydration, heat stress, and getting sunstroke were indicated. After prompting, by asking about possible appearancerelated disadvantages, parents perceived getting a wrinkled skin, freckles, and (hyper) pigmentation or spots as being general negative aspects of the sun. With regard to negative aspects of the sun on wellbeing, parents indicated the heat from sunrays was sometimes excessive.

On the other hand, parents often mentioned particularly perceiving the sun as yielding positive rather than negative aspects, and extensive lists of benefits were reported throughout all interviews. Positive perceptions mentioned were health-, well-being-, and appearance-related. Health-related perceptions reported were the sun being the reason to be outside more and children being more physically active, fit, living a healthy life, and receiving sufficient vitamin D: "And for me, I really get energy by sitting in the sun". Parents perceived being in the sun as considerably important and part of everyday life. Mental well-being was perceived evidently and positively affected by the sun, as parents observed an improved mood for themselves as well as their children when being in the sun. Several aspects, like feeling happy and joyous, energetic, being more socially active, and enjoying the warmth on the skin, were mentioned: "... it is also just a general feeling of happiness".

\subsection{Perceptions regarding a tanned skin}

One theme that frequently emerged without prompting, was appearance-related. The majority of parents perceived a tanned skin, resulting from sun exposure, as being positive, pretty, and more pleasant and healthier than pale skin, in both adults and children: "In my experience, I think people (with a tanned skin) look more fit, more alive, yes, healthier", and a pale skin was often associated with illness, being unhealthy, or a lack of nutrition. Interestingly, one parent particularly mentioned a pale skin as being prettier than a tanned skin: "I think that it (pale skin) is pretty. Prettier, yes, I really like it better than a tanned and wrinkled $s k i n "$. The positive perceptions regarding a tanned skin were often reinforced by friends or family members complimenting parents or their children on the way it looks, especially after a holiday. Additionally, a tanned skin was not perceived as positive when it was the result of sunbed use, with some parents using the term "sunbed-tanned" to explain their negative perceptions accordingly, along with being unhealthy, tacky, or not attractive. Moreover, parents considered the extent to which a skin is tanned as being important: "It depends on the gradation; if it becomes like a super-tanned skin, I don't think that that's healthy at all". Despite the positive perceptions, parents stated that they did not intentionally strive after a tanned skin for their children and a tan often occurred by chance. However, several statements of parents indicated that their preference for a tanned skin may play a role in performing sun protection measures for their child: "... though I'm probably thinking about it unconsciously, since I kind of like a bit of a tan", and "... deep in my heart I think it looks nice. But then I think oh, uh, I only rub them in with sunscreen at noon and not in the morning for example. It (leaving the child unprotected in the sun before noon) is possible". 


\section{Perceptions regarding sun protection}

All parents perceived sun protection as being important, were aware and well-informed about different sun protection measures, and performed both direct and indirect sun protection behaviours. Furthermore, mothers most often facilitated sun protection measures in advance of a situation (e.g. buying sunscreen, bringing sunglasses), whereas fathers were often initiators of sun protection during sun exposure situations. Nonetheless, all parents in a relationship perceived their parental role in their children's sun protection of equal importance and regarded it as a result of teamwork with their spouse. Motivational factors for parental sun protection performance were most often a light (or lighter) skin type, a history of sunburn, and knowing people who had had, or still had, a form of skin cancer.

\subsection{Direct sun protection behaviours}

Parents applied sunscreen, with SPF50, often as a singular and predominant method, even though they were well aware of the importance of sun avoidance and wearing protective clothing: "Yes, no, it's not that I take clothing into account. No, I mean, it is adequate to use sunscreen if you apply it sufficiently (...). Well, you could think of wearing sunglasses and that sort of thing... But principally it is sunscreen". However parents perceived a large number of barriers concerning sunscreen application, such as ambiguity about the working mechanisms of sunscreen (e.g. protection duration, recommended amount of sunscreen, or whether sunscreen is waterproof): "... I don't know, did I have to reapply (the sunscreen) every 30 minutes instead of every hour? For me, this is very difficult", or "Yes, if I can be absolutely sure that it (water-resistant sunscreen) would work effectively for 2-3 hours, I would definitely buy it".

Shade-seeking behaviour as an additive to sunscreen was the second most practiced behaviour, with parents indicating using trees, parasols, beach tents, or staying inside as protection measures for their children. Although specifying that seeking shade was important, parents often only sought shade when practicing a particular activity, such as eating or resting, or after their children's skin had already turned red. Parents perceived barriers with sun avoidance since shady areas were often not available at venues where their children were playing: "...there is a chance that there is not any shade at all (when being outside). And it's uhm, for hours and hours".

Even though putting on clothes was performed the least, some parents mentioned (buying) UV-protective clothing specifically, without prompting. "I once bought a UV-protective swimsuit or something like that (...), but it remains in the closet with the price tag still on it". Several parents indicated the use of T-shirts on very sunny days, although clothing was considered as too hot and uncomfortable for children, especially when they were playing near the waterfront, and it being a hassle and impractical for parents: "... Maybe that thought is wrong, but yeah, the children also don't like wearing long-sleeved clothing...". However, wearing hats was frequently mentioned among younger children. Although sunglasses were rarely used, some parents mentioned that their child liked wearing sunglasses since "... her mom and dad wear them too...".

\subsection{Indirect sun protection behaviours}

All parents performed indirect sun protection by stimulating or supporting their children to execute sun protection themselves, such as informing children about the importance of sun protection, demonstrating sun protection behaviours (e.g. by roleplaying with dolls), and actively teaching children to perform sun protection. The children's age at which parents started supporting children in practicing sun protection themselves varied widely (e.g. roleplaying at the age of 5 or putting sunscreen in the schoolbag from the age of 10). None of the parents had thought about future directions or plans concerning teaching their children sun protection behaviours: "Oh, well, I haven't made any (future) plans to be honest (laughing)".

Although parents played an important role in children's sun safety, most children were, to a certain extent, able to perform sun protection behaviours themselves as well. The age at which children started executing behaviours themselves varied widely, with e.g. children starting to apply sunscreen or putting on sunglasses themselves ranging between 5 and 12 years old. Where some young children were independently applying sunscreen (7 years: "... meanwhile they do it themselves, and if I forgot it, they would bring sunscreen to me, for example, and then say 'we still have to do this'..."), some older children did not perform sun protection 
themselves (11 years: "No, we usually still do that... He is not very active in that area"). No clear distinction between boys and girls was apparent, although some parents perceived girls as initiating sun protection from a younger age than boys. Overall, parents indicated that most children enjoyed performing sun protection behaviours themselves as it made them feel grown-up, cool, and comfortable: "We put the sunscreen in her backpack and then she really uses it. She is really trying her best and likes doing it". Furthermore, parents preferring to be in control of their children's sun protection often performed all sun protection behaviours until their child was older, whereas parents specifying their children's own responsibility stimulated children's own sun protection from an earlier age: "Well, you can't do much about it, right? So, I hope she learns from us that it is important to use sunscreen and that she thinks of it, and uses it, herself (laughing)."

\section{Perceptions regarding sunburn}

Although all parents mentioned taking sunburn precautions, almost all children (96\%) had experienced at least one case of sunburn: "...the sun shines and then... she comes inside with especially red, really red cheeks, the cheeks are a bit discoloured...". Although not specifically asked for, twelve parents reported sunburn in the previous summer months. A few parents explicitly mentioned having been sunburnt themselves during their childhood and definitely not wanting their child to suffer as well: "I mean, at very short notice it could be really, really painful right? I got really badly sunburnt myself and it's painful". Parents described sunburn situations either explicitly or implicitly, according to their recall of experienced or imagined sunburns. Additionally, the distinction between sun exposure being either anticipated or unanticipated in the occurrence of sunburns was revealed as being important.

\subsection{Explicit and implicit sunburn situations}

Eighteen parents mentioned that they were able to explicitly recall one or more situations in which their child experienced sunburn. All of these parents reported feeling guilty and shocked as soon as they noticed the sunburn: "... But I didn't pay enough attention and therefore she was suffering from it for the next few days and I felt sorry for her." When asked about the severity of children's sunburns, it appeared difficult for parents to define the sunburn: "Hmm, not really burnt. I didn't think she was, that she was suffering from it. No painful sunburn. But I thought she was a bit too red on her calves..." or "Because sometimes shade is not enough to prevent sunburn. Well sunburn, at least deeper tanning..." In some cases, parents initially stated that no sunburn had occurred, whereas after introducing one or two prompts, they did recall a sunburn situation. Parents mentioned water-related situations being a great challenge for various reasons, such as the water rinsing off sunscreen, children playing on the waterfront and refusing appliance or reappliance, or characteristics in the physical environment impeding sun protection (e.g. absence of shade). Several parents mentioned the temperature as an indicator for necessary sun protection measures: "But in the Netherlands I often think: ah well, it's not that bad at 23 degrees (Celsius)...", or: "... and if it's very hot sometimes, applying sunscreen only once may not be sufficient ...". The amount of cloudiness was also mentioned as making it difficult to estimate the UVR-strength: "If it's a bit cloudy for example, you don't feel like 'Oh, let's put on a hat', while the sun can still be very strong. That makes it so difficult".

Seven parents could either not recall their child having been sunburnt, or indicated that their child had never experienced sunburn, after which the researchers asked the parents to imagine a sunburn situation. Nonetheless, these parents were able to provide detailed information and examples regarding several potential sunburn situations and related barriers. The researchers identified these situations as implicit sunburn situations. Moreover, the observation emerged that these parents did not recognize sunburn as such, even though previously provided information proved otherwise, as illustrated by the following examples: "... somewhat reddened skin, but not really burnt with blisters or other distress, so not really burnt, no", and "Because, yes, it was red, but fortunately not burnt". These parents stated that they imagined it would be challenging not being present to have control over sun protection (e.g. their child being at school or at a friend's house), since teachers or other caretakers could be unaware about adequate sun protection measures. Second, situations in which children were engaging in outdoor activities (e.g. playing, biking, or at sports clubs), were considered to be difficult since the focus of the situation simply did not concern sun protection. Third, parents acknowledged several barriers related to the climate or the physical environment, 


\subsection{Anticipated and unanticipated situations}

When parents expected to be out in the sun, the majority took precautions by applying at least one sun protection method to protect their children's skin. Despite this, parents frequently mentioned being surprised by the strength of the sun, resulting in sunburn. Parents described feeling that their child's sunburn occurred suddenly, and that they were shocked by the rapidity of the event: "Yes, it went faster than I thought. Maybe the power of the sun (UV index) was too strong and I think I underestimated that". During this expected sun exposure, parents were not able to intervene earlier since the critical point in time was too abrupt: "...lt took me by surprise. It (sunburn) went faster than I'd expected".

Furthermore, unexpected sun exposure occurred especially when the sun exposure lasted longer than anticipated or prepared for: "Uhm but yes, sometimes they are having so much fun ... and then uh, yes, then you sometimes forget that (initiating sun protection)". In some cases, parents did not know their child was outside at all. When sun exposure was unexpected or not anticipated, parents did not bring sufficient sun protection products with them or their children were not shielded by protective clothing. Moreover, parents mentioned certain situations as not being associated with sun protection, such as when children engage in activities outside parental supervision when going to a friend's house or school: "...and then you're walking to school and you suddenly remember: oops, we forgot to use sunscreen". Parents believed that having sun protection materials ready at all times could have helped them to prevent future cases of sunburn: "Actually, having a backpack ready for sunny days, yes, and also with sunscreen in it, yes. And we should take that bag with us".

\subsection{Facilitators}

Besides barriers affecting adequate children's sun protection, parents also mentioned facilitating factors. With regard to health education, parents mentioned that increasing societal awareness regarding skin cancer risk and sun safety policy at schools and communities (e.g. sports clubs) would facilitate children's sun protection. Moreover, receiving up-to-date alerts regarding the current UV index via (online) weather forecasts, mobile apps, or on signs at recreational or public venues were mentioned. Almost all parents mentioned that they were in need of information about sufficient sun protection methods. Besides information targeted directly at them, parents felt that media campaigns could be helpful as well. Some parents believed that detailed information regarding their children's sun safety would have been helpful when their children were much younger (e.g. at the child health clinic or at day-care centres). Including children themselves in educational efforts was also described as facilitating.

Regarding health promotion, almost all parents mentioned shade availability at public venues as an important facilitator for sun protection behaviour, along with signs reminding them to take sun protection measures. Moreover, since parents were often insecure about sufficient sun protection at school, shady areas in school playgrounds were often mentioned. Some parents also mentioned provision of sunscreen at public venues, such as at restaurants: "... make sure that at restaurants a bottle of, eh, SPF50, is available. So that anyone can use it".

\section{Discussion}

This study provides a comprehensive exploration of parental perceptions regarding sun exposure, sun protection, and sunburn. Additionally, situations in which children experienced sunburn revealed in-depth information, presenting potential directions for future prevention efforts.

Overall, almost all children, of which seven parents did not explicitly report one, had experienced at least one case of sunburn. The high prevalence of children's sunburn occurred although all parents reported their intentions to perform sun protection behaviours, as well as the use of at least one sun protection measure. Moreover, the application of sunscreen was the most frequently reported protection measure. The 
preference for sunscreen in this sample is in accordance with existing literature $(76,156)$, although almost all parents reported difficulties in adequate application and regarded sunscreen use as complex. Other sun protection behaviours (e.g. shade-seeking or wearing clothes) were executed less often, with parents perceiving them as even more challenging, which has also been reported previously $(117,189)$. The most frequently mentioned situations in which sunburn occurred were situations involving water, a lack of shade, cloudiness and low temperatures causing underestimation of the UV index, and being caught by surprise.

There were positive perceptions regarding a tanned skin, for both adults and children, among a majority of the parents, as well as in their social environment. The influence of desiring a tanned skin can affect sun protection choices $(190,191)$, with earlier studies revealing that parents with positive attitudes toward tanning tend to protect their children less adequately $(51,55,161)$. This was not apparent in this study. Nonetheless, further investigation concerning the extent to which these attitudes affect sun protection behaviours is necessary. More importantly, the presence of normative beliefs approving and complimenting a tanned skin should be changed, since social norms have been found to influence parental sun protection behaviours as well $(118,192)$. In any case, modifying parental attitudes toward tanning in order to improve parental sun protection behaviours is highly recommended.

Although parents in this study were knowledgeable about general skin cancer risk, and motivated to perform sun protection behaviours, misconceptions concerning sun safety were still frequently detected. First, parents reported a reliance on sunscreen as sole prevention method, which could potentially explain the high prevalence of sunburn found in this study (193-196). Moreover, parents felt insecure and experienced ambiguity in the correct use of sunscreen. Sun safety recommendations emphasize the use of multiple sun protection methods (96). Since the lack of clear and consistent communication regarding sufficient sunscreen use has been drawn attention to earlier (6), educating parents about the necessity for adequately performing multiple sun protection behaviours is warranted. Second, parents often reported inaccurate estimations of the temperature and UV index, resulting in inadequate preparations for sun exposure situations. The confusion and lack of awareness concerning the relation between UV index, cloud coverage, and the need for sun protection measures among parents has been previously described (115, 161). Specifically, since parents were not always well-prepared for these weather conditions, awareness of the increased risk of sunburn in specific weather conditions is needed. Lastly, with regard to parental selfefficacy to perform sun protection, this study revealed that parents felt insecure, and perceived performing sun protection behaviours correctly as difficult. It is known that experiencing difficulties and low levels of self-efficacy influence parental sun protection behaviours negatively $(55,119,131)$. Further research to identify specific difficulties and barriers regarding sun protection is recommended in order to design sun safety interventions and teach parents adequate (coping) skills (197).

Second, characteristics in the physical environment, such as situations in which limited shade was available, and especially at public venues involving water, were perceived as particularly burdensome in adequately protecting children (77). Since the availability of shady areas increases the likelihood of shadeseeking behaviour $(166,167)$, the importance of shade at recreational areas should be recognized. Moreover, warning signs involving the current UV index, along with specific advice for sun protection measures at public venues, could be beneficial.

Although some parents indicated that their children were, to some extent, able to perform sun protection measures themselves, none of the parents reported future plans for teaching their children sun protection behaviours. Previous studies revealed that children are able to enhance their own sun protection behaviours (156) and that attitudes in favour of sun protection decline when children become older $(79,98)$. Stimulating parents to teach their children to adequately practice sun protection behaviours themselves is crucial in order to increase the establishment of sun protection throughout life $(71,155)$.

While the high number of reported cases of sunburn may demonstrate that parents were willing to talk straightforwardly and honestly about their experiences, most parents had initial difficulties in mentioning sunburn explicitly. For example, at first, several parents indicated that either no sunburn had occurred after which they elaborately mentioned explicit factors causing sunburn. In addition, some seemed to minimize 
the severity of their children's sunburn or had difficulty distinguishing 'deep tanning' from sunburn. On the one hand, this could potentially be explained by a tendency to provide socially desirable responding (52) and therefore attenuating the severity of sunburn. Parents mentioned feeling guilty and shocked when their child experienced sunburn, a finding congruent with a recent study (163). On the other hand, the initial underreporting of sunburn could be explained by parents not recognizing the classification of sunburn as such. The validity and reliability of self-reported sunburn have shown to be a great challenge, as illustrated in earlier work (198), although not specifically among parents. Hence, further exploration concerning this crucial topic is warranted to decide upon future intervention development. Furthermore, parents indicated that previous cases of sunburn among themselves and their children caused them to enhance their sun protection behaviours, which is in line with previously reported findings $(195,199)$. Nevertheless, contrasting findings in which previous cases of sunburn do not serve as potential cues to action for future sun protection behaviour are known as well $(52,55,199)$. Therefore, the influence of actual experience of sunburn in future sun protection behaviour needs further investigation.

\section{Strengths and Limitations}

This study has various strengths. To the best of our knowledge, this study is the first to explore parental perceptions regarding sun exposure, sun protection behaviour, and sunburn among Dutch parents. The study provides an essential exploration that can guide future quantitative research and intervention development. The qualitative origin of this study enabled a broad understanding of parental perceptions regarding various themes. Since semi-structured interview guides were used (200), respondents provided elaborate input. This resulted in comprehensive information regarding extensive themes (e.g. parents positive attitude toward tanning).

Second, efforts were made to enhance heterogeneity in the sample of respondents. Although representativeness and generalization of samples used in qualitative studies is not possible (201), the influence of certain demographic characteristics (i.e. parental sex, age, and educational level, and children's sex and age) on sun protection behaviour was acknowledged by striving for inclusion of parents with different backgrounds $(178,179)$.

Third, to optimize the validity and reliability of this study, various strategies were applied. Multiple researchers worked closely together throughout the process, standardized topic guides and observation notes were used to minimize potential biases (182), and all interviews were critically reflected upon. Moreover, crosschecking of coding and calculating the inter-coder reliability were performed, showing an almost perfect level of agreement (187).

Fourth, the chances of a social desirability bias may have been diminished by performing the interviews by telephone, rather than face to face, especially given the sensitivity of some questions (202).

Although this study has several strengths, a few limitations should be mentioned as well.

First, with regard to participant selection, a response and selection bias could have occurred (178), resulting in a decreased representativeness of the sample. Since parents had to actively express interest in participation, demonstrated high levels of sun safety awareness, and were in general highly educated, sun protection behaviours and sunburn patterns may be different than those of less motivated and lower educated parents, as revealed in a study in which children of lower educated parents were less protected and more frequently sunburnt than those of higher educated parents (203). Moreover, the relatively low retention rate could have induced selection of motivated parents.

Second, although the telephone interviews could have diminished possible social desirability, this bias cannot be ruled out (204). As illustrated in the results of this study, several parents initially indicated that their child had not suffered sunburn, as the interview progressed, the opposite turned out to be the case. The effects of a possible social desirability bias are expected to be minimal, given the high prevalence of 
reported sunburn in this study.

Third, execution of parental sun protection behaviours was assessed using subjective, rather than objective measures. Together with a social desirability bias, the sensitivity of the themes could have induced a parental overestimation of their sun protection behaviours, as is also suggested in earlier work $(78,205)$. To validate a potential over reporting among parents, studies comparing self-reported and objectively measured sun protection could be beneficial (206).

\section{Conclusions}

Educating parents about adequate preparation of sun exposure situations as well as specific weather-and environmental conditions should be considered in future sun safety interventions. Additionally, given its limited use among the parents in this study, a focus on wearing UV-protective clothing and other garments is also worthy of attention. Furthermore, a focus on environmental cues encouraging sun protection behaviours, such as increasing the availability of shady areas or warning signs concerning the UV index in the physical environment is recommended. Moreover, future research is recommended to investigate whether underreporting or underestimation of sunburn that appeared in this study is a result of either parental hesitation to admit to a child's sunburn or misconceptions about classifying sunburn. In any case, educational efforts to raise awareness and acknowledgement concerning the UV index, sunburn occurrence and severity, importance of simultaneously performing multiple sun protection behaviours, and focusing on teaching parents skills to overcome barriers, are warranted to enhance parental and children's sun safety. Additionally, reducing the desire to have a tanned skin among both parents and children needs further consideration. 
Appendix Chapter 2

Table 2. Themes, codes and sub-codes

\begin{tabular}{|c|c|c|}
\hline Themes & Codes & Sub-codes \\
\hline \multicolumn{3}{|l|}{ 1. Sun exposure } \\
\hline \multirow[t]{12}{*}{ Negative perceptions } & Health & Sunburn \\
\hline & & Skin cancer \\
\hline & & Skin damage \\
\hline & & Heat stress/sunstroke \\
\hline & & Dehydration \\
\hline & & Headaches \\
\hline & Well-being & Feels (too) warm on skin \\
\hline & & Feeling unpleasant \\
\hline & Appearance & Wrinkles \\
\hline & & Pigmentation spots \\
\hline & & Dry skin \\
\hline & Other & Mosquitos \\
\hline \multirow[t]{15}{*}{ Positive perceptions } & Health & Vitamin D \\
\hline & & More energy/being more fit \\
\hline & & Beneficial for biorhythm \\
\hline & & Beneficial for muscles \\
\hline & & $\begin{array}{l}\text { Beneficial for skin diseases (e.g. } \\
\text { psoriasis) }\end{array}$ \\
\hline & & Waking up more easily \\
\hline & & Being physically active \\
\hline & Well-being & Feeling good \\
\hline & & Feeling happy \\
\hline & & Feeling of general well-being \\
\hline & & Feeling relaxed \\
\hline & & Experiencing freedom \\
\hline & & Feeling more at ease \\
\hline & & A holiday feeling \\
\hline & & Feeling less depressed \\
\hline
\end{tabular}




\begin{tabular}{|c|c|c|}
\hline & Appearance & A tanned skin \\
\hline & & Wearing pretty clothes \\
\hline & - A tanned skin & \\
\hline & Negative & Degree of colouring \\
\hline & & Sunbed-tanned \\
\hline & Positive & Healthy \\
\hline & & Pretty \\
\hline & & Self-confidence \\
\hline & Neutral & \\
\hline & - Children's tanned skin & \\
\hline & Negative & Degree of coloured skin \\
\hline & Positive & Healthy \\
\hline & & Beautiful \\
\hline & & Fresh \\
\hline & Neutral & \\
\hline & Children's own perception & Funny \\
\hline & - Social norm & \\
\hline & Positive & Healthy \\
\hline & & Beautiful \\
\hline & & Compliments \\
\hline & Intentional tanning & \\
\hline & - Pale skin & \\
\hline & Negative & Unhealthy \\
\hline & & Sick \\
\hline & & Not beautiful \\
\hline & & Spending too little time outdoors \\
\hline & - Other & \\
\hline & Positive & Beautiful \\
\hline & Neutral & Sensitive skin \\
\hline & & Long-lasting days \\
\hline & & Flowers \\
\hline
\end{tabular}




\begin{tabular}{|c|c|c|}
\hline & & Appealing environment \\
\hline & & Friendly and happy people \\
\hline Themes & Codes & Sub-codes \\
\hline \multicolumn{3}{|l|}{ 2. Sun protection } \\
\hline \multicolumn{3}{|l|}{ Parent-for-child } \\
\hline \multirow[t]{8}{*}{ Direct } & Sunscreen application & Prior to sun exposure \\
\hline & & Sun Protection Factor (SPF) \\
\hline & & Routine use \\
\hline & Seeking shade & \\
\hline & Sun avoidance & \\
\hline & Clothing & Hat \\
\hline & & UV-protective clothing \\
\hline & & Sunglasses \\
\hline \multirow[t]{8}{*}{ Indirect } & Supportive behaviour & Providing information \\
\hline & & Helping to remember sun protection \\
\hline & & Performing sun protection together \\
\hline & & Let children do it themselves \\
\hline & & Modelling \\
\hline & & Checking \\
\hline & & Teaching routine \\
\hline & & Providing sun protection materials \\
\hline \multirow[t]{3}{*}{ Partner-for-child } & $\begin{array}{l}\text { Similar to partner's sun } \\
\text { protection }\end{array}$ & Consistency \\
\hline & & Teamwork \\
\hline & $\begin{array}{l}\text { Different from partner's sun } \\
\text { protection }\end{array}$ & Organization \\
\hline \multirow[t]{7}{*}{ Child itself } & Sunscreen application & \\
\hline & Clothing & \\
\hline & Seeking shade & \\
\hline & $\begin{array}{l}\text { Child's perception of own sun } \\
\text { protection behaviour }\end{array}$ & Interesting \\
\hline & & Pleasant \\
\hline & & Cool \\
\hline & & Habit \\
\hline
\end{tabular}




\begin{tabular}{|c|c|c|}
\hline & $\begin{array}{l}\text { Differences between boys and } \\
\text { girls }\end{array}$ & \\
\hline & & Girls: younger age, boys: older age \\
\hline \multirow[t]{20}{*}{ Barriers for sun protection } & $\begin{array}{l}\text { Barriers in the physical } \\
\text { environment }\end{array}$ & Absence of shade \\
\hline & & Waterfront far away from shady area \\
\hline & Cognitive barriers & No priority \\
\hline & & No preparations/no habitual behaviour \\
\hline & & Unaware of UV index and weather \\
\hline & & Lack of knowledge \\
\hline & & Misconceptions \\
\hline & & Focus on other activity (e.g. sports) \\
\hline & Economic barriers & Price of sunscreen or clothing \\
\hline & Climate/weather & Wind \\
\hline & & Water \\
\hline & & Cloudiness \\
\hline & Sunscreen barriers & Type of sunscreen \\
\hline & & Warranted SPF \\
\hline & & Interval of reapplication \\
\hline & & Thickness of sunscreen \\
\hline & & Adverse effects of sunscreen \\
\hline & & Water resistance \\
\hline & & Relying on sunscreen only \\
\hline & Other & $\begin{array}{l}\text { Sun protection measures being } \\
\text { impractical }\end{array}$ \\
\hline \multirow[t]{6}{*}{$\begin{array}{l}\text { Facilitators for sun } \\
\text { protection }\end{array}$} & $\begin{array}{l}\text { Facilitators in the physical } \\
\text { environment }\end{array}$ & Shady areas \\
\hline & & Warning signs \\
\hline & & Information provision \\
\hline & & $\begin{array}{l}\text { Availability of other items (e.g. } \\
\text { sunscreen) }\end{array}$ \\
\hline & Cognitive facilitators & Adequate preparation \\
\hline & Social facilitators & Changed social norm \\
\hline
\end{tabular}




\begin{tabular}{|c|c|c|}
\hline & Political facilitators & Marketing \\
\hline & & $\begin{array}{l}\text { Innovative strategies (e.g. colouring } \\
\text { sunscreen bottle) }\end{array}$ \\
\hline & & $\begin{array}{l}\text { Information provision (e.g. via media, at } \\
\text { schools, day-care centres, sports clubs) }\end{array}$ \\
\hline & Other & Skin type \\
\hline & & History of sunburn \\
\hline & & Skin cancer experience \\
\hline & & Experience from other countries \\
\hline Themes & Codes & Sub-codes \\
\hline \multicolumn{3}{|l|}{ 3. Sunburn } \\
\hline \multirow[t]{21}{*}{ Explicit situations } & Locations with parents & Swimming pool or beach \\
\hline & & While playing \\
\hline & & While biking \\
\hline & & While in the garden \\
\hline & Locations without parents & At school \\
\hline & & At a friend's place \\
\hline & Location of sunburn & Calves \\
\hline & & Legs \\
\hline & & Shoulders \\
\hline & & Arms \\
\hline & & Face \\
\hline & & Back \\
\hline & Severity of sunburn & \\
\hline & Timing & Morning \\
\hline & & Afternoon \\
\hline & & Spring \\
\hline & & Summer \\
\hline & Duration of sun exposure & \\
\hline & Weather conditions & Temperature \\
\hline & & Cloud coverage \\
\hline & & Awareness prior to situation \\
\hline
\end{tabular}




\begin{tabular}{|c|c|c|}
\hline & & Awareness during situation \\
\hline & Adults present & \\
\hline & Parents' feelings/emotions & Shock \\
\hline & & Guilt \\
\hline & Awareness of sunburn & During the situation \\
\hline & & After the situation \\
\hline & Sun protection methods & None \\
\hline & & Sunscreen \\
\hline & & Seeking shade \\
\hline & & Clothing \\
\hline & & Hat and sunglasses \\
\hline & & Colouring skin as indicator \\
\hline Implicit situations & Situations with parents & During holidays \\
\hline & & While playing \\
\hline & & While biking \\
\hline & Situations without parents & While at a sports club \\
\hline & & While playing \\
\hline & & While at school \\
\hline & Timing & Spring \\
\hline & Duration of sun exposure & \\
\hline & Weather conditions & Temperature \\
\hline & & Cloud coverage \\
\hline & & Awareness prior to situation \\
\hline & & Awareness during situation \\
\hline
\end{tabular}


chapter three

\section{Identification of relevant socio-cognitive determinants explaining multiple parental sun protection behaviours}


'A theory that denies that thoughts can regulate actions does not lend itself readily to the explanation of complex human behavior.'

- Albert Bandura 


\section{Background}

Adequate sun safety during childhood is crucial for decreasing skin cancer risk in later life. Although parents are an essential target group in applying sun protection measures for their children, insight into the determinants associated with their sun protection behaviours is limited. This study aims to identify most relevant determinants in predicting multiple parental sun protection intentions and behaviours in different sun exposure situations.

\section{Methods}

A longitudinal survey study with two measurements was conducted among Dutch parents $(\mathrm{N}=670)$ of children (4-12 years old). Twenty-seven socio-cognitive determinants were examined in terms of relevance regarding four parental sun protection behaviours in different sun exposure situations. The CIBER approach was used to visualize room for improvement (sample means) on all determinants and their association strengths (correlations) with sun protection intentions and behaviours.

\section{Results}

Behaviour-specific rather than generic determinants were most relevant in explaining all sun protection behaviours. Of these determinants, attitude, self-efficacy and action planning, and especially parental feelings of difficulty in performing sun protection behaviours, were most relevant. Altogether, the explained variance of all socio-cognitive determinants was highest for shade-seeking behaviour $\left(R^{2}=.41\right.$ and $.43)$ and lowest for supportive behaviour $\left(R^{2}=.19\right.$ and .29) in both planned and incidental sun exposure situations respectively.

\section{Discussion \& Conclusions}

This study provides detailed insight into relevant socio-cognitive determinants of parental sun protection behaviours in various sun exposure situations and directions for composing parental skin cancer prevention interventions. Future sun safety interventions should emphasize on enhancing parental feelings of self-efficacy, especially for shade-seeking and clothing behaviours. 


\section{Introduction}

Skin cancer incidence, and especially melanoma, is rising excessively worldwide (146). The burden of melanoma is highest in Europe, North-America and Oceania, where together 84\% of incidences in 2018 occurred (207). Incidence rates are expected to rise even further over the coming decades in fair skinned populations (208), emphasizing the importance of prevention efforts. Exposure to Ultraviolet Radiation (UVR) and sunburn incidence, particularly during early childhood, are the most important risk factors in the aetiology of melanoma $(41,42,209)$. Globally, one to two thirds of children experience at least one sunburn every year $(48,50-53,154)$. Therefore, it is warranted to reduce the amount of received UVR and sunburn occurrence by performing adequate sun protection behaviours (e.g. using sunscreen, seeking shade) in children specifically.

Parents play a crucial role in directly or indirectly protecting their children from overexposure to UVR and sunburns. Young children generally rely on their parent's sun protection behaviour towards them (156). As they grow older, parents serve as their primary role models by which children learn how to perform these behaviours themselves (210). Moreover, studies investigating the parental role in sun protection reveal that parents are important gatekeepers in encouraging children's own sun protection attitudes and behaviours $(51,80,157,211)$. Parents are therefore essential targets to promote sun protection in children.

Although guidelines exist to adequately protect ones skin against UVR $(6,212,213)$, reported parental adherence varies among studies. For example, studies report that only $17 \%$ of parents perform sun protection among their children correctly (74), or $75 \%$ of parents inadequately apply sunscreen to their child (76), while other studies describe high parental performance of sun protection behaviours $(78,79)$. Sun protection ideally comprises simultaneous application of multiple protective measures (6), with seeking shade and wearing protective clothing increasingly being recommended. However, sunscreen use is often the most preferred, and regarded as the safest precaution taken by the general population $(194,214)$ and among parents $(76,156)$. Nonetheless, an overreliance on sunscreen can occur, resulting in an increased risk of unprotected UVR-exposure and sunburn $(193,215,216)$. This sunscreen paradox is particularly perceived during planned sun exposure (e.g. when going to the beach). However, results may differ for situations in which people are not purposely exposed to sun (e.g. when walking or cycling) (215). Hence, sun protection approaches need to address comprehensive sun protection behaviours in various situations (217).

Understanding why parents engage in sun protection behaviours by examining relevant socio-cognitive determinants is fundamental in order to address these determinants in interventions (197). Various studies have investigated the role of socio-cognitive determinants (e.g. attitude, social norms) in the onset of sun protection behaviours among adults $(129,218,219)$, but comprehensive studies investigating parent-forchild sun protection behaviours are limited. These studies often focus on pre-motivational determinants such as knowledge or risk-perceptions $(116,132)$, or attitude $(75,116,117)$, include parents of very young $(2$ to 6 years old) children $(118,119,130)$, or have sunscreen use as main outcome $(133,220)$. Although preliminary, results of these studies indicate an influential role of socio-cognitive determinants such as anticipated regret, attitude, self-efficacy, and action planning in parental sun protection behaviours. In order to target sun safety interventions for parents, identification of the most relevant determinants foregoing various parental sun protection behaviours is imperative $(110,131)$. Gaining comprehensive insight by investigating the relevance of both generic (e.g. knowledge about skin cancer) and behaviour-specific (e.g. attitude towards clothing behaviour) socio-cognitive determinants is warranted.

Despite the importance of sun safety during childhood and the powerful parental role in initiating sun protection behaviours $(80,156)$, targeted interventions are so far restricted. Moreover, those interventions targeted at parents, report limited effects on parental behaviour, such as clothing or seeking shade (143, $158,159,221,222)$. This is also illustrated by systematic reviews concerning effectiveness of educational interventions, revealing that conclusions about effectiveness regarding parent-for-child sun protection behaviours could not be drawn (82), reported limited efficacy (158) or did not specifically report on parental target groups (143). A recent review discussed the limitation of available evidence among children's 
caregivers when assessing effectiveness of community-wide sun safety interventions (223). Although some positive effects of educational interventions on parental sun protection behaviours are reported, strong conclusions about effectiveness of these parent-for-child interventions remain absent due to lack of data. Hence, development of interventions targeted at comprehensive parental sun protection behaviours is warranted.

This study aims to identify most relevant generic and behaviour-specific socio-cognitive determinants regarding their room for improvement and association with both direct (i.e. sunscreen use, clothing, seeking shade) and indirect (supporting the child in performing sun protection measures) parent-for-child sun protection behaviours. Further, this study distinguishes relevance of socio-cognitive determinants in both planned (e.g. going to the beach) and incidental (e.g. cycling) sun exposure situations.

\section{Methods}

\section{Study design}

Data from a longitudinal cohort study regarding parental sun protection with a total of four measurements was used. For this study specifically, data from the second (October 2016 (T1)) and third (October 2017 (T2)) measurement was analysed, as all determinants (T1) and behaviours (T2) relevant for this study aim were included. This study was exempted from approval by a medical ethics committee, since participants were not exposed to medical procedures or behavioural demands (224). The data collected in this study was pseudonymized, meaning that the research team could not identify specific persons within the dataset (225). STROBE guidelines for observational research were followed to report this study (226).

\section{Participants and recruitment}

The Dutch research organization TNS-KANTAR (227) invited an eligible sample of parents that were members of an existing research panel, representative of the Dutch general population based on education and income. Parents were eligible for participation if they at least one child in the primary school age. Online informed consent was obtained by TNS-KANTAR. The sample of parents received one invitational email and one reminder per measurement. In these invitations, a direct link was provided to the online questionnaires assessing direct (i.e. sunscreen use, clothing and seeking shade) and indirect (supportive) sun protection behaviours and related behavioural determinants. Parents were asked to answer the questions regarding the same child (the youngest in their household) during both measurements. After completion of each questionnaire, parents received a small incentive consisting of gift vouchers.

\section{Measurement}

The online survey assessed the following aspects: 1) demographic characteristics, 2) execution of sun protection behaviours, 3) generic determinants, and 4) behaviour-specific determinants. Section 3 and 4 were based on the pre-motivational, motivational and post-motivational phases of the I-Change model (162), an integrative theoretical framework for understanding health behaviour.

\section{Demographic characteristics}

Age, sex and educational level of parents were assessed, together with age and sex of their child. Educational level of parents was categorized as low (1; e.g. primary education)/medium (2; e.g. secondary vocational education)/high ( 3 ; e.g. university education), conform guidelines of Statistics Netherlands ( 228 , 229). Children's age was classified into three groups ( 4 to 6 years, 7 to 9 years and $>10$ years; cf. Dutch primary school system). 


\section{Direct and indirect sun protection behaviours}

Direct sun protection behaviours consisted of 1) applying sunscreen, 2) providing the child with UVprotective clothing and/or garments and 3) seeking shade. Indirect behaviour consisted of supporting the child in conducting his or her own sun protection behaviours (defined as advising, facilitating sun protection behaviours and checking/monitoring whether the child applied sun protection behaviours). The frequency of self-reported application of these behaviours was assessed regarding the past summer season, using a 5-point Likert scale ranging from 'never' (1) to 'always' (5). A non-applicable answer category was included for indirect behaviour; in case the child was too young to be supported in his or her own sun protection behaviour.

Execution of all sun protection behaviours was assessed for two different types of sun exposure. Firstly, planned sun exposure (PS), consisting of situations during which parents and/or their child expected and intended to be exposed to the sun (e.g. going to the swimming pool or beach) and, secondly, incidental sun exposure (IS) comprised situations of unintentional sun exposure (e.g. cycling or playing outside).

In total, eight outcome measures (three direct behaviours and one indirect behaviour; all in two situations) were assessed. In order to clarify the distinction of behaviours and situations, parents received explanation about the separate sun protection behaviours beforehand, according to guidelines from the Dutch Cancer Society (73) and examples of different sun exposure situations.

\section{Generic socio-cognitive determinants}

Generic socio-cognitive determinants were assessed universally across all four behaviours. Knowledge consisted of 14 true-false statements regarding UVR exposure, sunburns and skin cancer ('correct' (1), 'incorrect' (0), or 'I don't know' (0)). Risk-perception consisted of 12 questions addressing cognitive (4 items) and affective ( 4 items) risk susceptibility, and severity ( 4 items) concerning both sunburns and skin cancer, addressing PS and IS situations. Anticipated regret contained 4 questions regarding regret parents feel when their child would experience a sunburn. Furthermore, the frequency of children's sunburns during both the previous summer season and across their lifetime was assessed as an indicator for cues to action. Lastly, parent's attitude toward the importance of their children's tanned skin was assessed. All items were assessed using a 5-point Likert scale.

\section{Behaviour-specific determinants}

Behaviour-specific socio-cognitive determinants were assessed for each separate sun protection behaviour, in which two questions, regarding PS and IS situations, were assessed per item. For every sun protection behaviour, attitude was measured by 2 items assessing the extent to which parents regarded the sun protection behaviour as important or unimportant, as well as pleasant or unpleasant. Social norm was measured by 2 items per behaviour, distinguishing the perceived norms based on the opinion of partners (if applicable) and important others. Self-efficacy was measured by 2 items per behaviour, which differentiated parental experiences of difficulty and ability of performing sun protection behaviours. Action planning was measured by 1 item per behaviour, assessing whether a specific plan was formulated to perform sun protection. Lastly, intention towards each specific sun protection behaviour was assessed by 1 item. Table 1 provides a set of exemplary items.

\section{Analyses}

Descriptive analyses were performed using IBM SPSS Statistics for Windows, Version 24.0 (230). Not applicable answers to questions were excluded from analyses. A sum score was computed of the number of correctly answered knowledge items (ranging from 0 (low levels of knowledge) to 14 (high levels of knowledge)), which was then recoded into a scale ranging from 1 to 5 to enhance visual comparison between all determinants imputed in further analyses (as they were assessed using 5-point Likert scales). For the analyses, determinants from T1 $(n=28)$ and behavioural outcomes from T2 $(n=8)$ were used. 
CIBER (Confidence Interval-Based Estimation of Relevance) was used to establish relevance of parental socio-cognitive determinants regarding their sun protection behaviours (231). CIBER is a data visualization method integrating descriptive statistics that combine two types of analyses: assessing (1) univariate distribution of each determinant (based on means), and (2) associations with behavioural outcomes (based on correlations). Univariate distributions show the room for improvement regarding each determinant (i.e., how high participants score on the scale). This needs to be combined with the association with behavioural outcomes, as those determinants that are associated with behaviour and where there is room for improvement, are the most relevant candidate variables to intervene upon. For both means and correlations, confidence intervals show the accuracy with which these can be estimated. CIBER visualizes this information to facilitate comparison on spatial dimensions, which is necessary when making selections for intervention development. Furthermore, visualization foregoes the apparent accuracy and objectivity produced by numbers. Given the relative width of most sampling distributions and the subsequent variation that occurs in estimates over samples $(232,233)$, caution in basing decisions on the exact computed numbers seems prudent. CIBER plots were created using the $R$ (234) package behaviorchange (235).

\section{Table 1. Exemplary items of behaviour-specific determinants concerning sunscreen use in incidental situations'}

\begin{tabular}{|c|c|c|c|}
\hline Determinants & & Items & $\begin{array}{l}\text { Answer categories and } \\
\text { coding }\end{array}$ \\
\hline \multicolumn{4}{|l|}{ Attitude } \\
\hline Importance & $\begin{array}{l}\text { Importance } \\
\text { of sunscreen } \\
\text { use }\end{array}$ & $\begin{array}{l}\text { 'When my child is engaging in outdoor } \\
\text { activities (e.g. playing, cycling) on } \\
\text { sunny days, I think that adequately } \\
\text { applying sunscreen to my child is (...)' }\end{array}$ & $\begin{array}{l}\text { (1. Not important }-2 \text {. } \\
\text { Slightly important }-3 \text {. } \\
\text { Moderately important } \\
-4 . \text { Important }-5 . \text { Very } \\
\text { important) }\end{array}$ \\
\hline Pleasantness & $\begin{array}{l}\text { Pleasantness } \\
\text { of sunscreen } \\
\text { use }\end{array}$ & $\begin{array}{l}\text { 'When my child is engaging in outdoor } \\
\text { activities such as playing, exercising, } \\
\text { cycling or walking on a sunny day, I } \\
\text { think adequate sunscreen use for my } \\
\text { child is (...)' }\end{array}$ & $\begin{array}{l}\text { (1. Not pleasant }-2 \text {. } \\
\text { Slightly pleasant }-3 \text {. } \\
\text { Moderately pleasant } \\
\text { - 4. Pleasant }-5 \text {. Very } \\
\text { pleasant) }\end{array}$ \\
\hline \multicolumn{4}{|l|}{ Social norm } \\
\hline Partner & $\begin{array}{l}\text { Partner's } \\
\text { opinion } \\
\text { about } \\
\text { sunscreen } \\
\text { use }\end{array}$ & $\begin{array}{l}\text { 'When my child is engaging in outdoor } \\
\text { activities such as playing, exercising, } \\
\text { cycling or walking on sunny days, my } \\
\text { partner thinks it is important that we } \\
\text { adequately use sunscreen for our child }\end{array}$ & $\begin{array}{l}\text { (1. Totally disagree }-2 \text {. } \\
\text { Disagree }-3 . \text { Neutral }-4 \text {. } \\
\text { Agree }-5 \text {. Totally agree - } \\
\text { 6. Not applicable }(=99))\end{array}$ \\
\hline Important others & $\begin{array}{l}\text { Opinion of } \\
\text { important } \\
\text { others about } \\
\text { sunscreen } \\
\text { use }\end{array}$ & $\begin{array}{l}\text { 'When my child is engaging in outdoor } \\
\text { activities such as playing, exercising, } \\
\text { cycling or walking on sunny days, } \\
\text { important people around me think it } \\
\text { is important that l/we adequately use } \\
\text { sunscreen for my child' }\end{array}$ & $\begin{array}{l}\text { (1. Totally disagree }-2 . \\
\text { Disagree }-3 . \text { Neutral }-4 \text {. } \\
\text { Agree }-5 . \text { Totally agree) }\end{array}$ \\
\hline
\end{tabular}




\begin{tabular}{|c|c|c|c|}
\hline \multicolumn{4}{|c|}{ Self-Efficacy } \\
\hline Difficulty & $\begin{array}{l}\text { Difficulty } \\
\text { to apply } \\
\text { sunscreen }\end{array}$ & $\begin{array}{l}\text { 'When my child is engaging in outdoor } \\
\text { activities (e.g. playing, cycling) on } \\
\text { sunny days, how difficult is it for you } \\
\text { to make sure he/she is adequately } \\
\text { protected with sunscreen?' }\end{array}$ & $\begin{array}{l}\text { (1. Very difficult }-2 \text {. } \\
\text { Difficult }-3 . \text { Neutral - } 4 \text {. } \\
\text { Easy }-5 . \text { Very easy) }\end{array}$ \\
\hline Ability & $\begin{array}{l}\text { Being able } \\
\text { to apply } \\
\text { sunscreen }\end{array}$ & $\begin{array}{l}\text { 'If my child is engaging in outdoor } \\
\text { activities (e.g. playing, cycling) on } \\
\text { sunny days, I am able to make sure } \\
\text { he/she is adequately protected with } \\
\text { sunscreen' }\end{array}$ & $\begin{array}{l}\text { (1. Definitely not }-2 \text {. } \\
\text { Probably not }-3 \text {. } \\
\text { Neutral }-4 \text {. Probably- } 5 \text {. } \\
\text { Definitely) }\end{array}$ \\
\hline \multicolumn{4}{|l|}{ Intention } \\
\hline & $\begin{array}{l}\text { Intention } \\
\text { to apply } \\
\text { sunscreen }\end{array}$ & $\begin{array}{l}\text { 'When your child is engaging in } \\
\text { outdoor activities (e.g. playing, cycling) } \\
\text { on sunny days, do you intend to } \\
\text { adequately apply sunscreen to him/ } \\
\text { her?' }\end{array}$ & $\begin{array}{l}\text { (1. Definitely not }-2 \text {. } \\
\text { Probably not }-3 \text {. Might - } \\
\text { 4. Probably- 5. Definitely) }\end{array}$ \\
\hline \multicolumn{4}{|c|}{ Action planning } \\
\hline & $\begin{array}{l}\text { Formulation } \\
\text { of action } \\
\text { plan(s) } \\
\text { to apply } \\
\text { sunscreen }\end{array}$ & $\begin{array}{l}\text { 'When your child is engaging in } \\
\text { outdoor activities (e.g. playing, cycling) } \\
\text { on sunny days, do you have a specific } \\
\text { plan to adequately use sunscreen for } \\
\text { him/her?' }\end{array}$ & $\begin{array}{l}\text { (1. Definitely not }-2 \text {. } \\
\text { Probably not }-3 \text {. Might } \\
-4 \text {. Probably would }-5 . \\
\text { Definitely would) }\end{array}$ \\
\hline \multicolumn{4}{|c|}{$\begin{array}{l}\text { Sun protection } \\
\text { behaviour }\end{array}$} \\
\hline & $\begin{array}{l}\text { Parent- } \\
\text { for-child } \\
\text { sunscreen } \\
\text { use during } \\
\text { the previous } \\
\text { summer } \\
\text { season }\end{array}$ & $\begin{array}{l}\text { 'When your child was engaging in } \\
\text { outdoor activities such as playing, } \\
\text { exercising, cycling or walking on a } \\
\text { sunny day during the previous summer, } \\
\text { to what extent did you adequately } \\
\text { apply sunscreen to protect your child?' }\end{array}$ & $\begin{array}{l}\text { (1. Never }-2 . \text { Rarely }-3 . \\
\text { Sometimes }-4 . \text { Often }-5 . \\
\text { Very often) }\end{array}$ \\
\hline
\end{tabular}

\section{Results}

\section{Sample characteristics}

At T2, 670 parents remained $(74.1 \%$ response rate; $58.5 \%$ mothers; $54.3 \%$ higher educated; Mean income range: 69.000 - 82.300) and were included in the analyses. Attrition analyses indicated that demographic characteristics were not significantly associated with drop-out on T1 and T2. From these parents, 339 (50.6\%) and $331(49.4 \%)$ answered the questionnaires regarding sun protection of respectively their son and daughter. Children were aged between 4 and 14 years (Modus=6; $M=8.8 ; S D=2.6$ ). Self-reported sunburns occurred at least once among $29.1 \%$ of the children during the previous summer season $(M=1.3 ; S D=.5)$ and among $77.4 \%$ of the children throughout their lives $(M=1.9 ; S D=.6)$. With regard to direct sun protection behaviours, sunscreen was frequently (i.e., 'Often' and 'Always') applied by parents in PS (88.2\%) as well as 


\section{Relevance of behaviour-specific determinants}

\section{Direct behaviours}

Overall, both beliefs assessing attitude demonstrated highest sample means regarding sunscreen use in both PS and IS situations. The belief regarding importance demonstrated higher mean scores and therefore less room for improvement than the belief about pleasantness of the sun protection behaviours. Moreover, for all sun protection behaviours, both attitudinal determinants indicated strong positive associations with both sun protection intentions and behaviours in PS as well as IS situations.

With regard to social norm, sample means were again higher for sunscreen use than for clothing and shadeseeking behaviour. The extent to which partners believe sun protection is important demonstrated highest mean scores for all behaviours, whereas the extent of importance among other people depicted lower mean scores, indicating more room for improvement. For all sun protection behaviours, the importance of a partner's opinion concerning the sun protection behaviour demonstrated positive associations with sun protection intentions and behaviours for all three behaviours, in both PS and IS situations. Compared to other determinants, the importance of sun protection according to other people often indicated the lowest associations.

Self-efficacy demonstrated lowest sample means compared to other determinants, especially for clothing and shade-seeking behaviour. Especially feelings of difficulty depicted lowest scores across almost all behaviours, indicating high perceived difficulty to perform sun protection behaviours and notable room for improvement. Compared to other behaviours, parents indicated highest difficulty for seeking shade. Moreover, being able to perform sun protection behaviours depicted notable room for improvement as well. Both aspects of self-efficacy demonstrated highest positive associations with intentions and performance across all behaviours, with feelings of ability to perform sun protection behaviours showing most positive associations.

Lastly, formation of action plans demonstrated low sample means and therefore opportunity for improvement for all sun protection behaviours in both PS and IS situations, with again seeking shade indicating the lowest scores across behaviours. Following self-efficacy, action planning often depicted the second highest association with intentions and behaviours to perform sun protection behaviours.

In Figure 1 an overview of the relevance of behaviour-specific determinants regarding direct sun protection behaviours is provided for both PS and IS situations. ${ }^{2}$

\footnotetext{
2 All CIBER plots described in this Chapter as well as CIBER plots visualizing relevance of sociocognitive determinants stratified by educational level, parental sex, children's sex and children's age and CIBER plots with sub-behaviours as outcomes can be retrieved from OSF (https://osf.io/vwr2g/)
} 


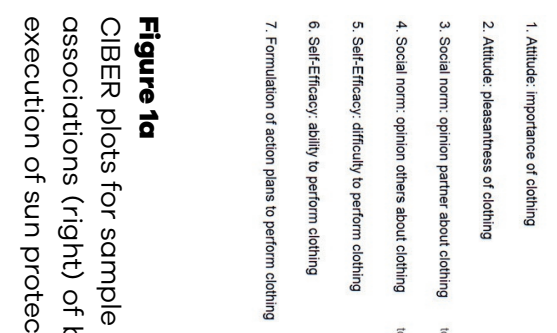

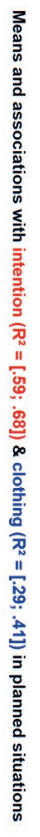

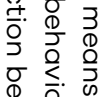

要

‥

足

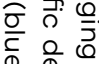

D

논.

要

כ.

方寺

贫

궁

ज.

定

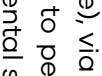

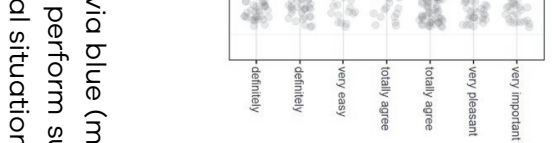

* o s a

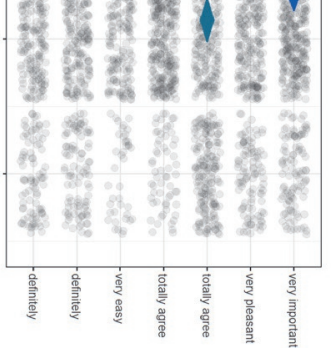

गे
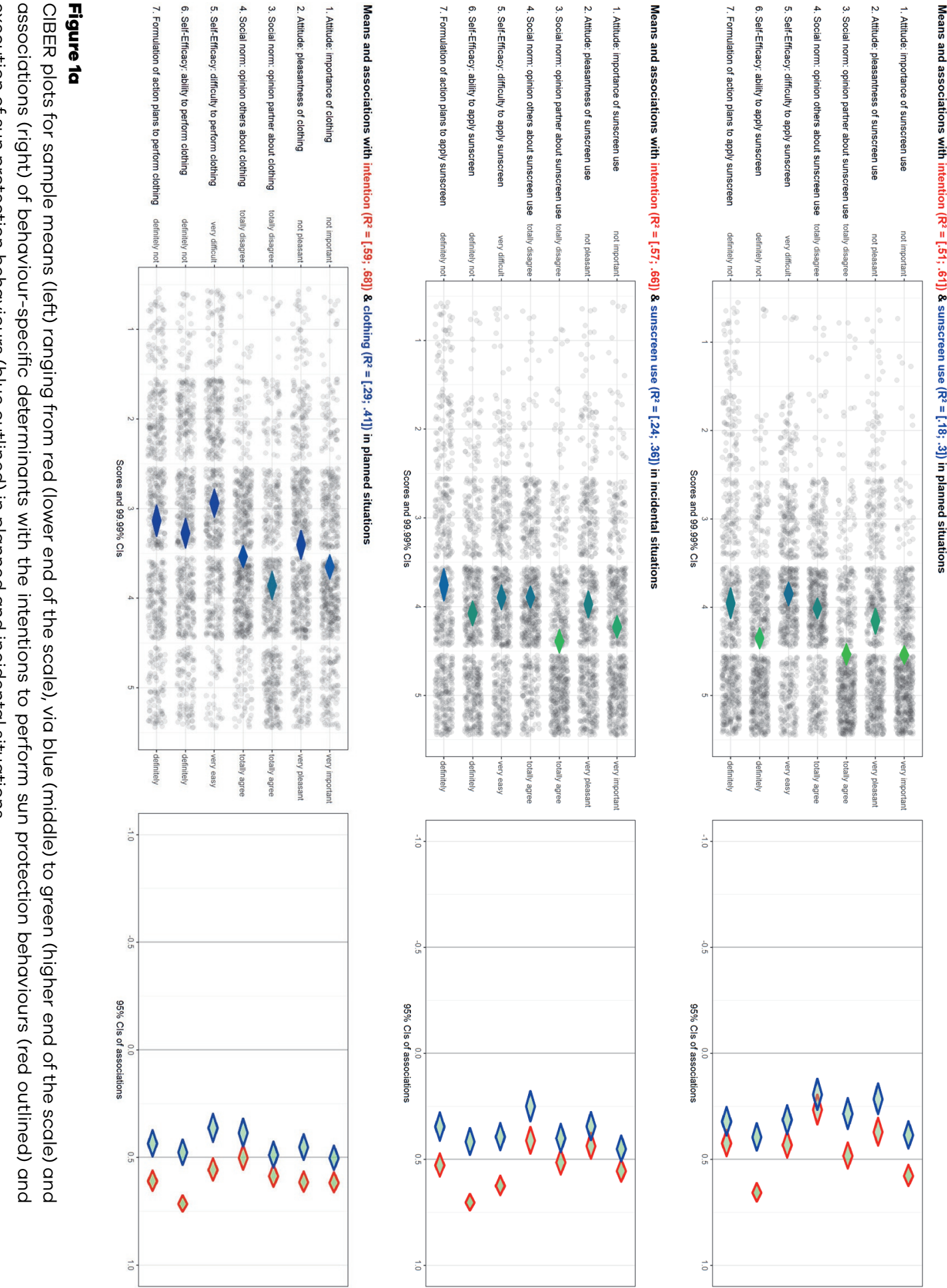

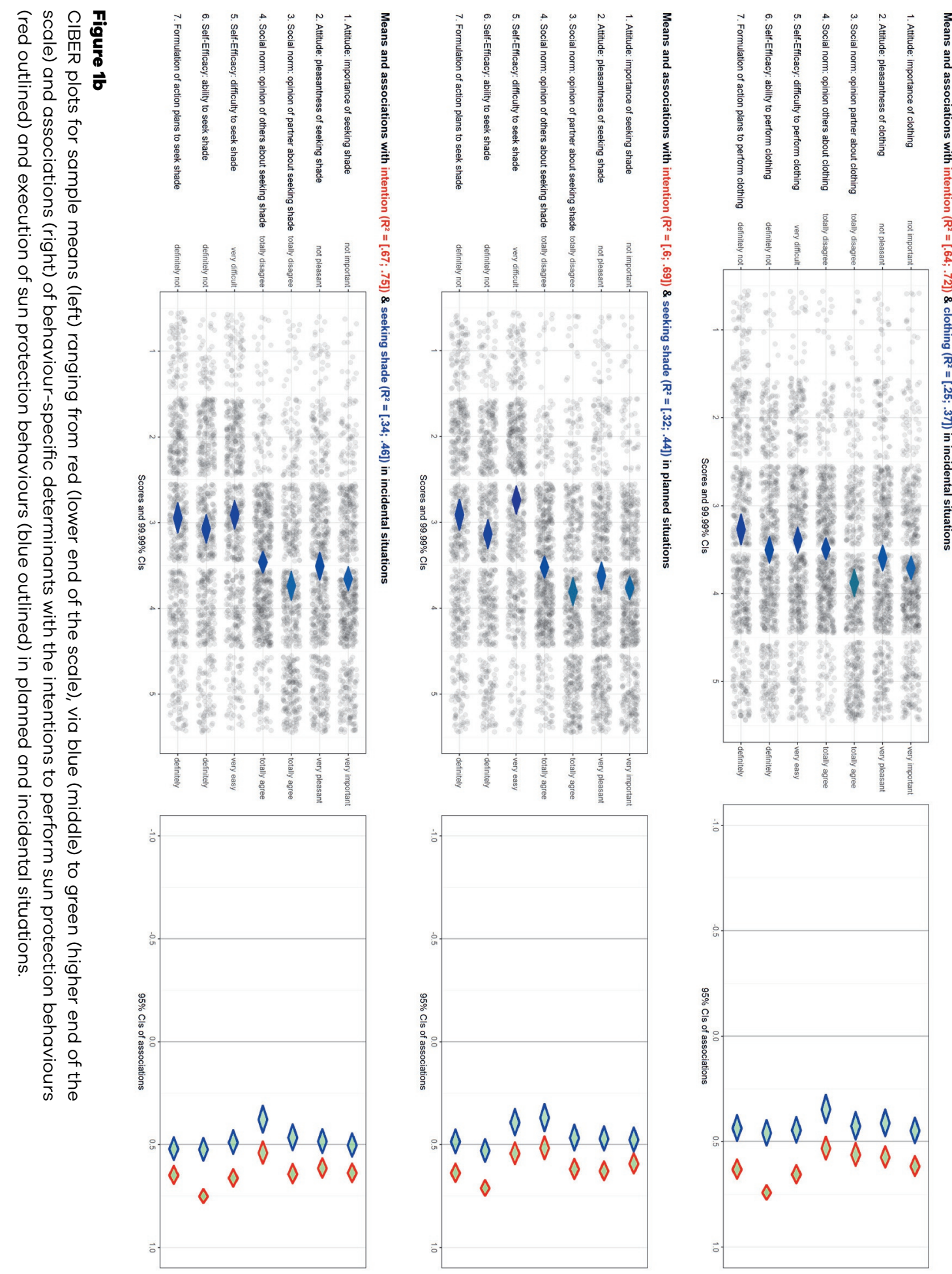

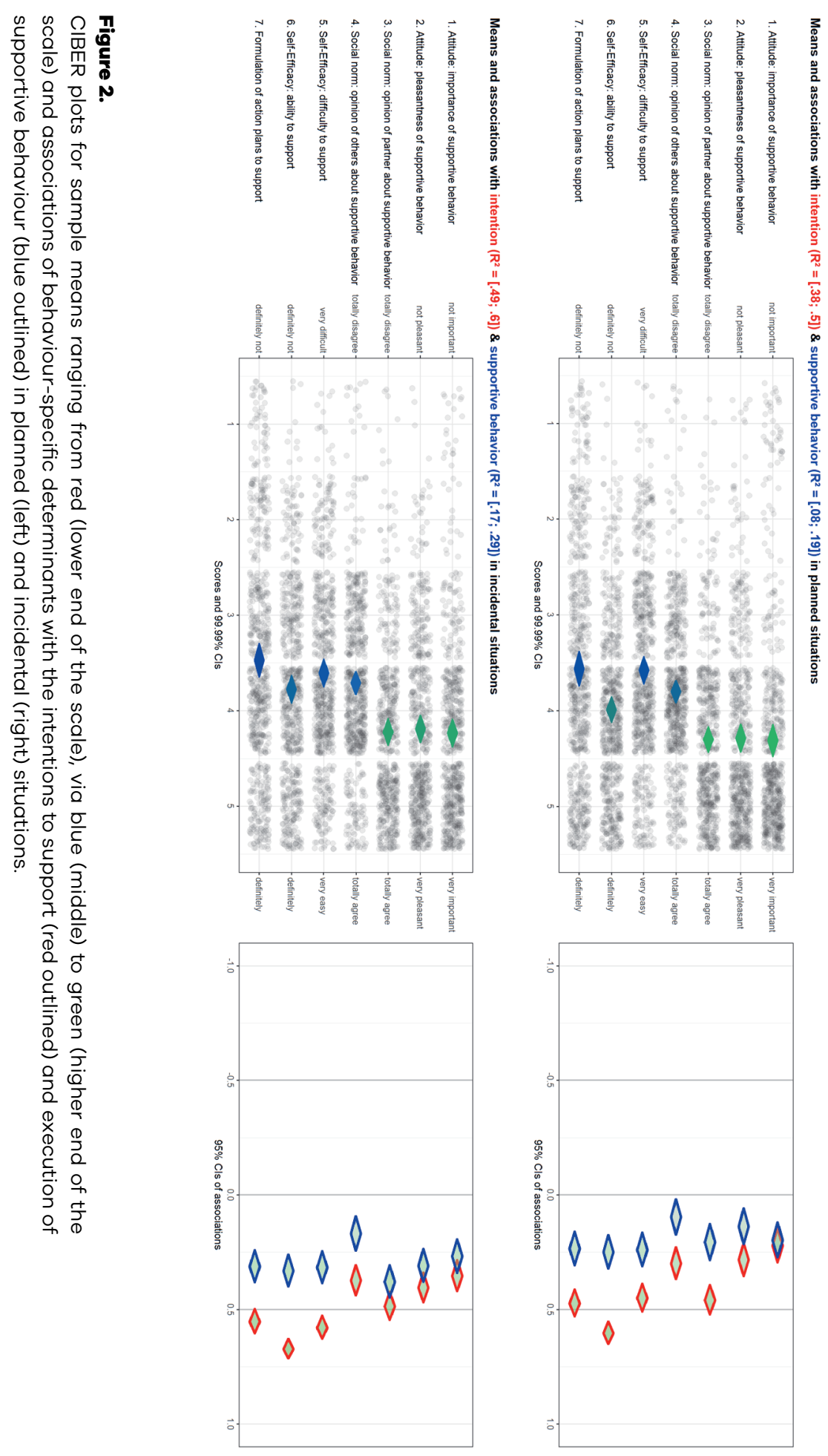


\section{Indirect behaviours}

Overall, behaviour-specific determinants foregoing supportive behaviour depicted comparable sample means in PS and IS situations. Moreover, sample means were highest for attitude and social norm (concerning partner's opinion) and lowest for self-efficacy (difficulty of providing support) and action planning. Associations with intentions and behaviours were most positive for determinants related to selfefficacy and action planning, with the self-efficacy belief about ability having the highest associations (Consider Figure 2). Since mostly older aged children are being encouraged to perform sun protection behaviours themselves, a smaller sample of parents reported execution indirect behaviour ( $n=637 ; 95.1 \%$ ).

\section{Relevance of generic determinants across all behaviours}

Knowledge portrayed high sample mean scores across all behaviours, whereas sample means regarding riskperception depicted lower scores. Particularly, lowest scores were reported for cognitive and affective risk susceptibility concerning skin cancer in IS situations. Moreover, higher sample means were demonstrated for determinants concerning skin cancer severity, with the severity of skin cancer in comparison with other cancer types depicting lowest mean scores. Anticipated regret was moderate to high across all behaviours, in which regret concerning sunburns was notably lower than regret concerning skin cancer development. The group mean for the attitude concerning children's tanned skin was low.

Associations of the generic determinants with intentions ( $r$-range $.07-.37$ ) and behaviours ( $r$-range .07 .34) were low and varied slightly across behaviours. Moreover, previous sunburns and the positive attitude towards children's tanned skin were negatively associated with all sun protection intentions ( $r$-range -.08 - -.30) and behaviours ( $r$-range -.01- -.30). Table 2 provides all sample distributions and associations with sun protection intentions and behaviours.

\section{Explained variance across all behaviours}

On average the full set of socio-cognitive determinants explained $19-41 \%$ of the variance in all sun protection behaviours in PS situations and $28-43 \%$ in IS situations, in which shade-seeking behaviour demonstrated highest and supportive behaviour lowest explained variance. The average explained variance for intentions to perform sun protection behaviours ranged between $46-66 \%$ in PS, and $58-73 \%$ in IS situations.

\section{Discussion}

This study provides detailed insight in relevant socio-cognitive determinants for predicting parental sun protection behaviours in various sun exposure situations. Relevance of determinants was indicated by both room for improvement, as well as their associations with sun protection intentions and behaviours. Overall, associations between generic determinants and sun protection intentions and behaviours were low, whereas behaviour-specific determinants were highly associated with these intentions and behaviours. Moreover, attitude, self-efficacy and action planning were particularly relevant regarding shade-seeking and clothing behaviours in both sun exposure situations. Additionally, determinants altogether showed greater relevance for explaining sun protection intentions and behaviours in incidental rather than planned sun exposure situations as well as for shade-seeking and clothing behaviour rather than for sunscreen use and indirect behaviour. 
Although the findings in this study clearly demonstrated the importance of behaviour-specific determinants, current educational sun safety interventions predominantly target generic (e.g. knowledge and riskperception in general) instead of behaviour-specific determinants, lacking evidence on long-term improved sun protection behaviours when directed at children $(82,136,158)$, or at parents $(117,143,158)$. Increasing knowledge and improving one's health beliefs only is evidently not sufficient for establishing health behaviour change $(109,236)$. With regard to skin cancer prevention specifically, skin cancer knowledge and awareness are not sufficient to establish sustainable sun protection behaviour (159). Focusing on additional behaviour-specific determinants in parental sun safety interventions is therefore highly recommended.

When thoroughly examining the relevance of specific socio-cognitive determinants, a few findings emerged. First, attitudes supportive of sun protection among parents seem important to include in interventions. Although in this study the room for improvement of attitude was lower than for other determinants, associations with all sun protection intentions and behaviours were strong. Parental attitudes are important in predicting various parent-for-child behaviours (237) and appear to strongly influence children's own attitudes with regard to sun protection (79). Second, self-efficacy regarding execution of sun protection behaviours was found to be essential. The positive association between parental self-efficacy and sun protection towards their children has been demonstrated before $(119,237)$. Besides, this study demonstrated the distinction between relevance of feelings of difficulty and ability. Notable room for improvement was especially shown regarding the experienced difficulty in performing sun protection behaviours. Investigating the reasons underlying of parental feelings of difficulty is essential for selecting specific behaviour change methods for intervention development (197). Although the larger project in which this study was conducted indicated difficult situations to perform sun protection (e.g. when it is too hot to wear clothing or when a child rejects to wear sunglasses), strong conclusions cannot be drawn. Moreover, confusion concerning recommended sunscreen application (195) or ambiguity about achieving sufficient vitamin D (163) could increase feelings of difficulty. Moreover, behaviour-specific determinants were more strongly associated with sun protection intentions than behaviours, implying the well-documented intention-behaviour gap (238). The relevance of action planning for all sun protection behaviours reported in this study, has been previously documented for parental sunscreen use (239). After understanding the particular difficulties to perform sun protection behaviours more clearly, directions for formulating specific action and coping plans can be integrated in future interventions to increase the likelihood of behaviour change $(240,241)$.

This study also found negative associations between determinants and intentions and behaviours. Parents reporting on their child having previously experienced sunburn(s), appear to subsequently apply less sun protection behaviours than parents whose child did not experience sunburn(s). Although the association between children's previous sunburns and future sun protection behaviour has not been thoroughly investigated, studies found positive correlations, indicating that sunburns function as a motivating factor for sun protection behaviour $(163,195)$. However, trends in sunburn occurrence remaining high over time have been reported $(49,216)$. Since sunburn experiences were assessed cumulatively in the current study, its negative association with sun protection behaviours could be caused by a behavioural pattern of noncompliance among parents. Furthermore, a negative association between a tan-favouring attitude and sun protection behaviours was apparent. Since the latter is corresponding with results in previous parentalfocused studies $(51,161)$, parental beliefs concerning a tan appearing healthy or pretty should be targeted in interventions to enhance sun protection practices.

Overall, variance in sunscreen use was less explained by determinants than shade-seeking and clothing behaviours. Since a strong parental preference for applying sunscreen among their children is known $(79,156)$, this could imply that sunscreen use originates by recitation and therefore becomes a habitual rather than reasoned or deliberately controlled behaviour (242). Since frequently performed behaviour can increase skill acquisition and reduce the impact of socio-cognitive determinants on intentions and behaviours $(236,243)$, behaviours can be triggered directly by certain cues in a situation in which the behaviour was performed in the past (244). Moreover, parents explicitly mentioned origination of habitual use of sun protection measures in situations where the behaviours were firstly established, such as going to the beach (220). This is also illustrated by the higher explained variance during IS situations in this study, indicating the more deliberately origination of sun protection behaviours in these situations. Understanding 
the role of automatic processes in sun protection behaviour may therefore also need further attention in future research (131). However, other determinants, not assessed in this study, could be relevant in predicting sunscreen intentions and behaviours, such as time perspective (preference of short-term over long-term health behaviour benefits) (245) and feelings of autonomy (246). Nevertheless, targeting sociocognitive determinants regarding shade-seeking and clothing intentions and behaviours in future sun safety interventions seems advantageous. Additionally, since the explained variance regarding indirect behaviours was the lowest across behaviours, and parental support and advice are essential in teaching children sun protection behaviours $(79,247)$, an emphasis on indirect behaviours in parental sun safety interventions is recommended. Ideally, both children and their parents should be included in sun safety interventions, since parental behaviours are closely related to children's own sun protection practices (51, $75,80)$.

There are a few limitations in this study that are worth mentioning. First, only parental determinants and behaviours were assessed. Although the parental perspective is highly relevant in understanding parentfor-child behaviours (237), children are increasingly able to perform health behaviours as they grow older (156) and are therefore important agents in sun safety interventions as well. Future studies could investigate children's behavioural determinants influencing their own sun protection behaviours. Second, the current study relied on parental self-reports. Although previous studies reveal positive correlations between parental self-reported and objectified sun protection behaviours $(248,249)$, future studies could consider objective assessment of behaviours to enhance validity of our findings. Lastly, the CIBER approach did not provide the opportunity to examine interactions between determinants and possible confounding factors. CIBER was however carefully selected since the advantages of combining both room for improvement and association strengths of determinants provide interesting directions for future intervention development.

To our knowledge, this study is the first to identify relevance of an extensive set of socio-cognitive determinants in predicting direct and indirect parental sun protection behaviours in different situations of sun exposure. The necessity of comprehensive sun safety interventions, targeting specific determinants and behaviours, is evident. In particular, a focus on self-efficacy in future interventions is strongly recommended, using behaviour change methods appropriate for this specific determinant (197). Enhancement of parental shade-seeking and clothing behaviours seems beneficial since socio-cognitive determinants illustrate a vital role in the prediction of these behaviours. Since this study demonstrated strong associations between specific parental determinants and their sun protection intentions and behaviours, composition of future sun safety interventions for children should strongly emphasize the parental role and influence within the family setting. 


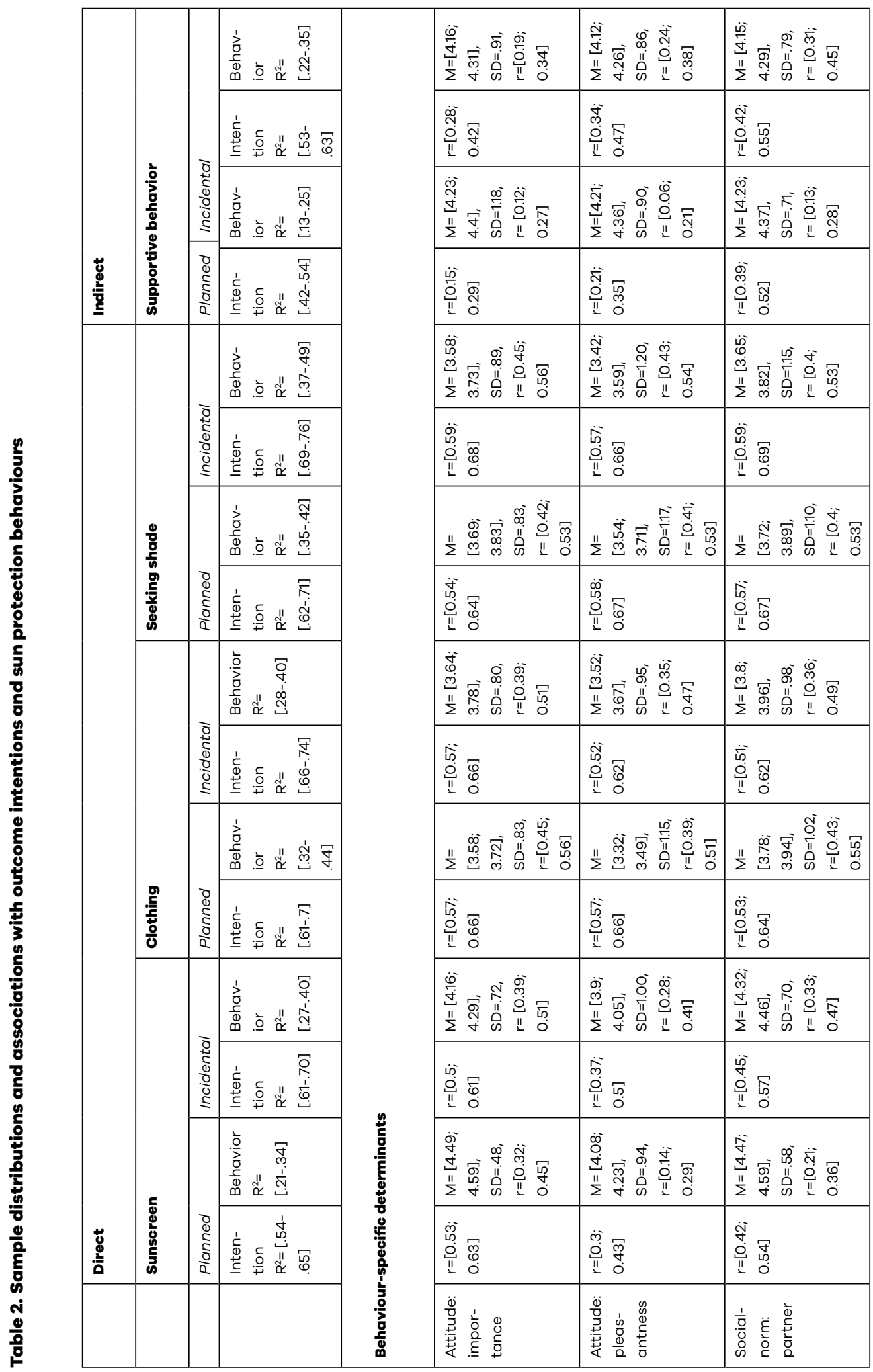




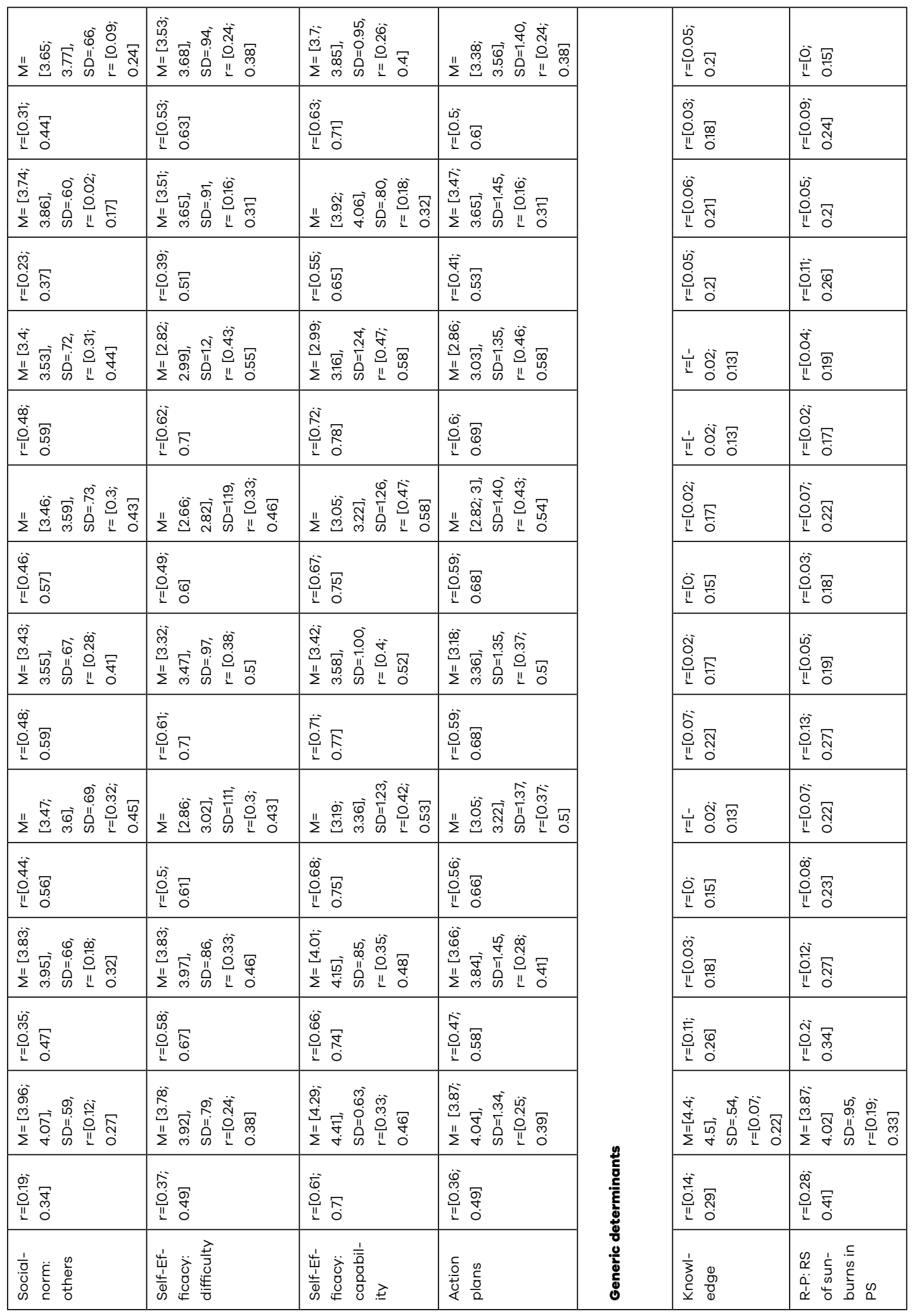




\begin{tabular}{|c|c|c|c|c|c|c|}
\hline 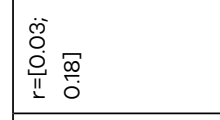 & 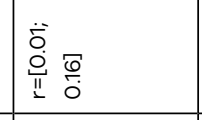 & 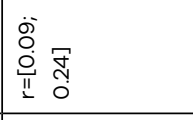 & 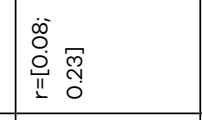 & 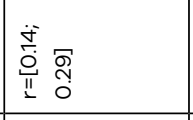 & 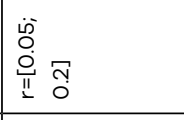 & 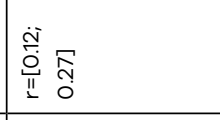 \\
\hline 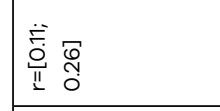 & 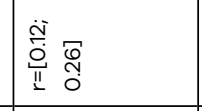 & 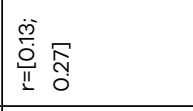 & $\begin{array}{l}\text { 言 品 } \\
\text { 恶 }\end{array}$ & 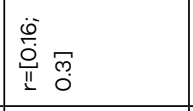 & 言 & 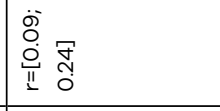 \\
\hline 엠정 & 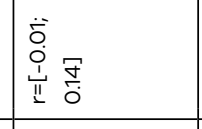 & 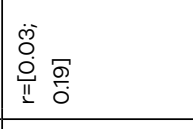 & 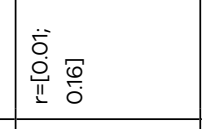 & 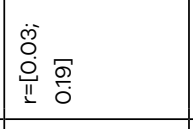 & 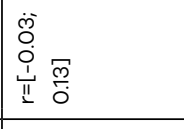 & 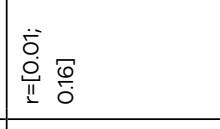 \\
\hline 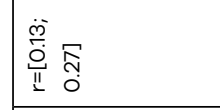 & 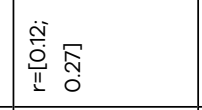 & 戀 & 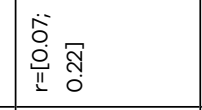 & 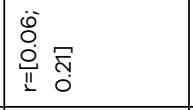 & 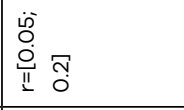 & 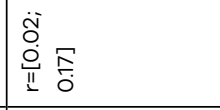 \\
\hline 突 & 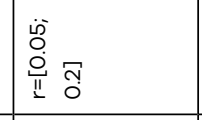 & 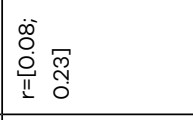 & 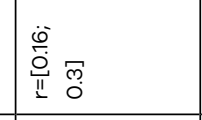 & 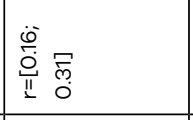 & 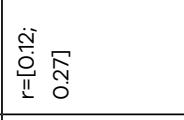 & 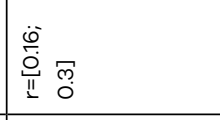 \\
\hline 突 & 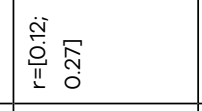 & 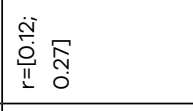 & 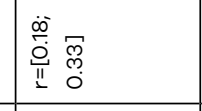 & 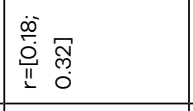 & 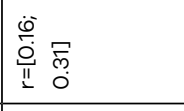 & 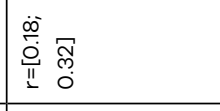 \\
\hline 言 & 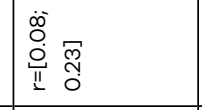 & 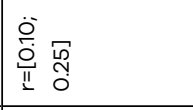 & 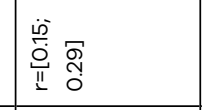 & 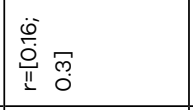 & 言竞 & 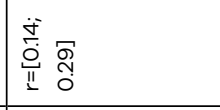 \\
\hline 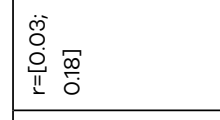 & 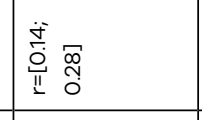 & 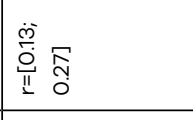 & 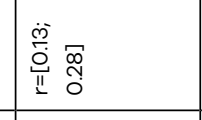 & 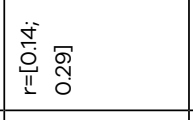 & 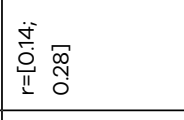 & 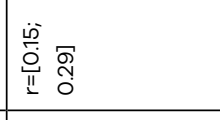 \\
\hline 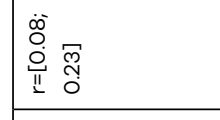 & 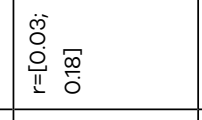 & 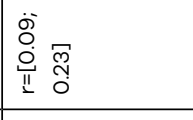 & 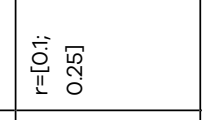 & 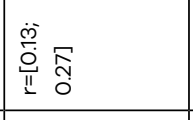 & 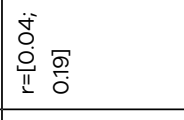 & 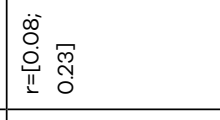 \\
\hline 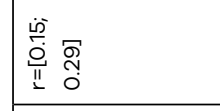 & 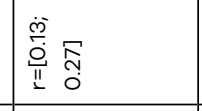 & 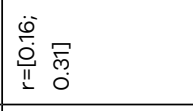 & 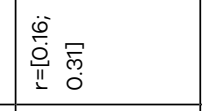 & 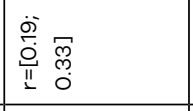 & 言曾 & 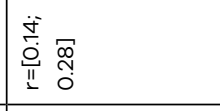 \\
\hline 言总 & 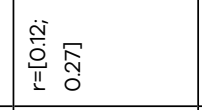 & 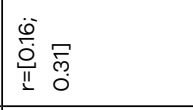 & 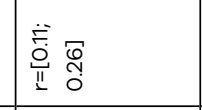 & 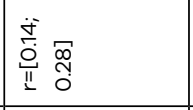 & 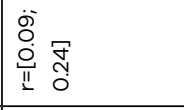 & 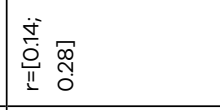 \\
\hline 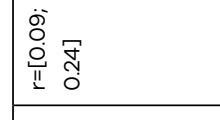 & 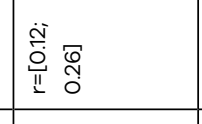 & 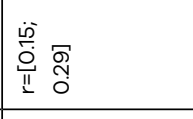 & 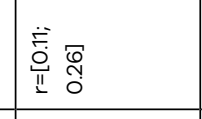 & 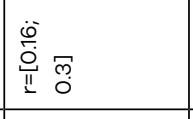 & 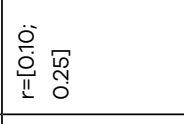 & 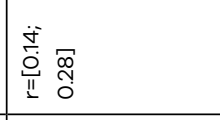 \\
\hline 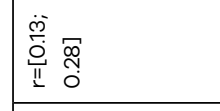 & 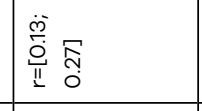 & 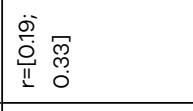 & 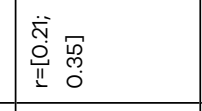 & 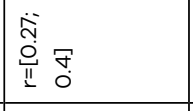 & 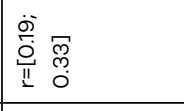 & 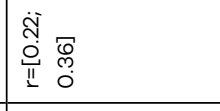 \\
\hline 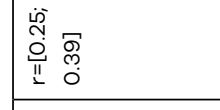 & 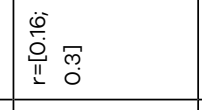 & 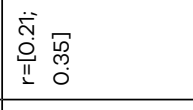 & 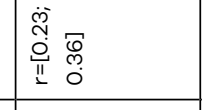 & 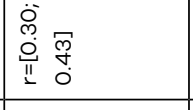 & 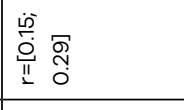 & 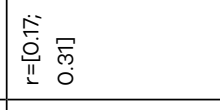 \\
\hline 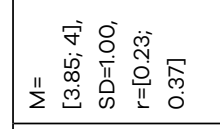 & 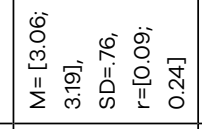 & 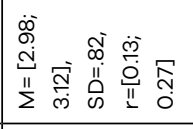 & 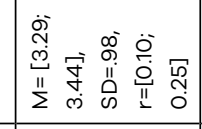 & 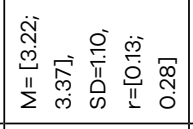 & 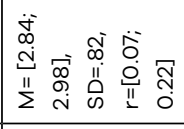 & 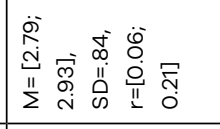 \\
\hline 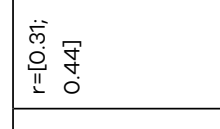 & 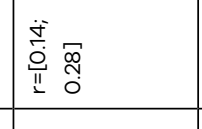 & 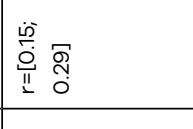 & 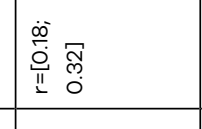 & 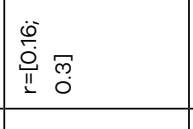 & 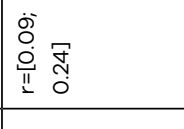 & 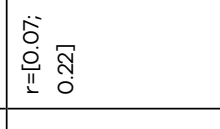 \\
\hline 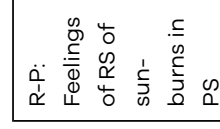 & 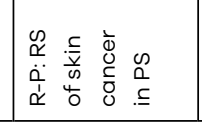 & 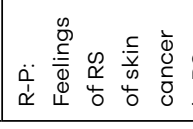 & 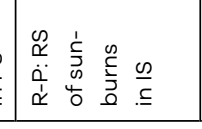 & 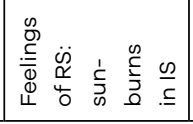 & 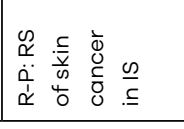 & 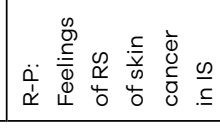 \\
\hline
\end{tabular}




\begin{tabular}{|c|c|c|c|c|c|c|}
\hline 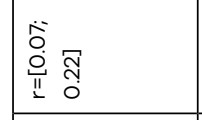 & 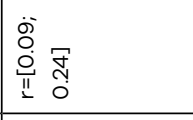 & 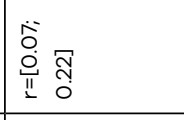 & 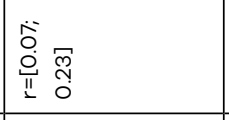 & 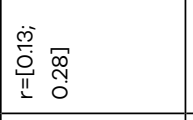 & 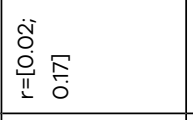 & 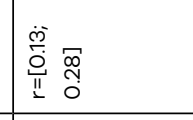 \\
\hline 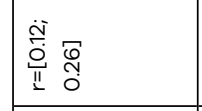 & 言㩊 & 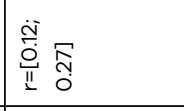 & 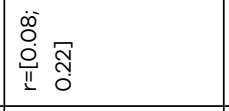 & 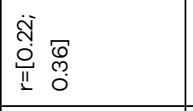 & 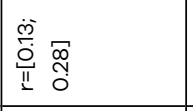 & 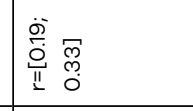 \\
\hline 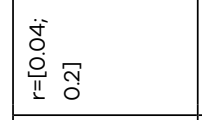 & 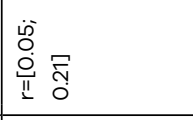 & \begin{tabular}{|l} 
\\
尊 \\
\end{tabular} & 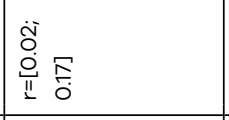 & 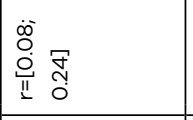 & 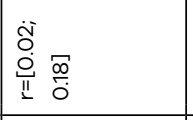 & 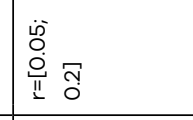 \\
\hline 童哭 & 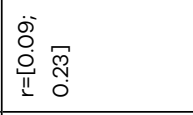 & 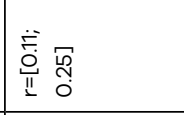 & 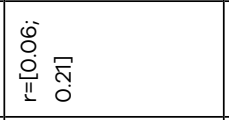 & 言局 & 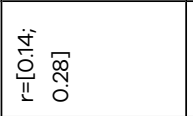 & 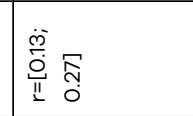 \\
\hline 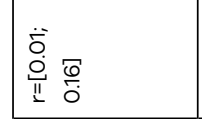 & 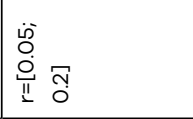 & 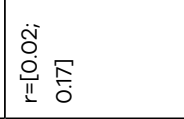 & $\frac{20}{0}$ & 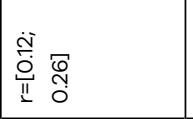 & 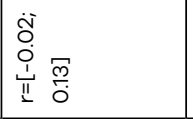 & 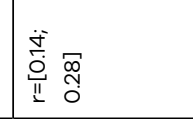 \\
\hline 落 & 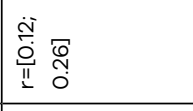 & 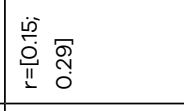 & 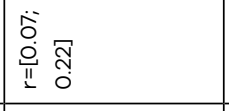 & 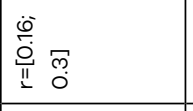 & 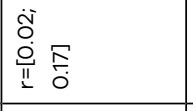 & 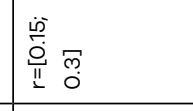 \\
\hline 产产 & 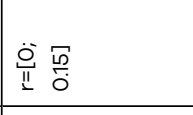 & 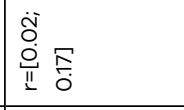 & 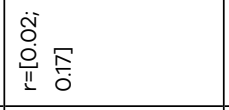 & 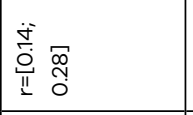 & 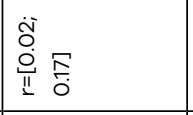 & 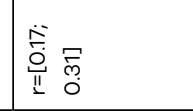 \\
\hline 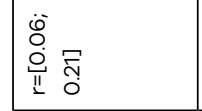 & 产 & 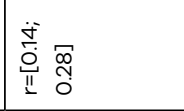 & 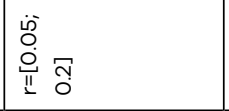 & 离 & 䓂 & 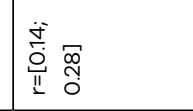 \\
\hline $\begin{array}{l}0 \\
\end{array}$ & 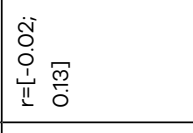 & 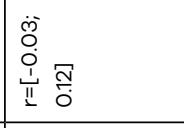 & 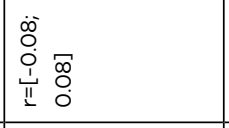 & 袋 & $\frac{20}{11}$ & 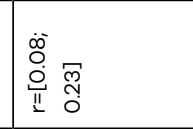 \\
\hline 言 & 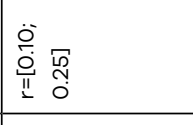 & 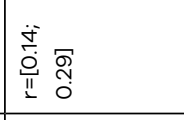 & 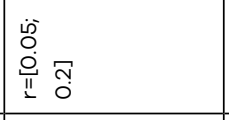 & 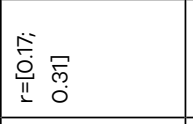 & 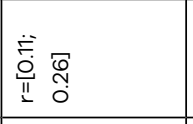 & 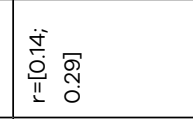 \\
\hline 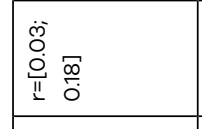 & 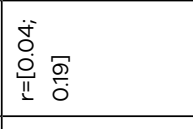 & 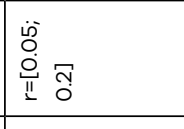 & 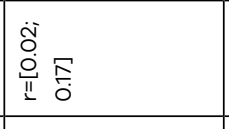 & 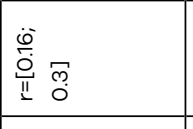 & 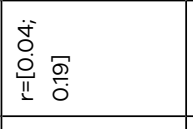 & 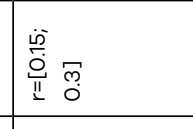 \\
\hline 产 & 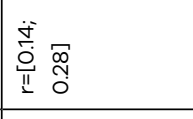 & 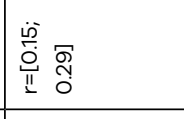 & 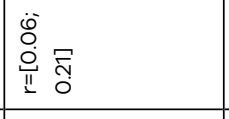 & 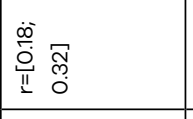 & 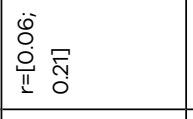 & 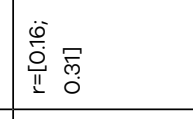 \\
\hline 言 & 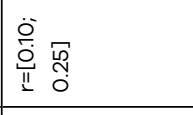 & 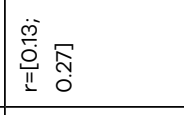 & 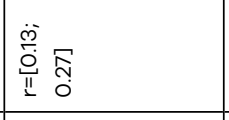 & 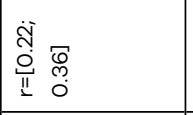 & 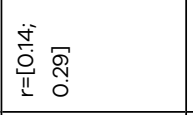 & 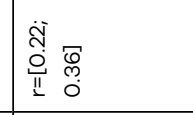 \\
\hline 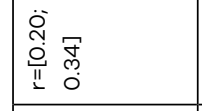 & 言要 & 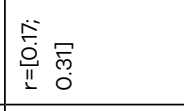 & 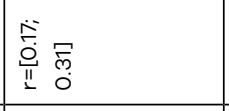 & 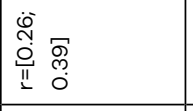 & 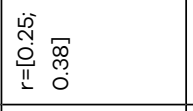 & 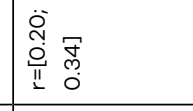 \\
\hline 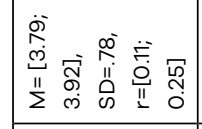 & 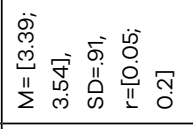 & 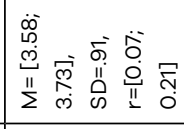 & 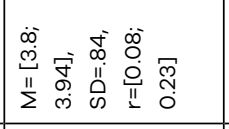 & 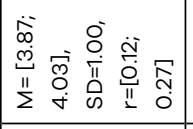 & 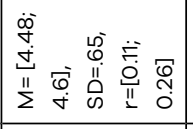 & 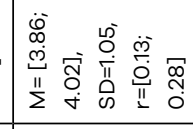 \\
\hline 亲 & 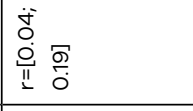 & 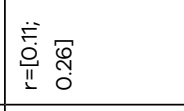 & 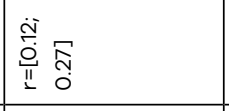 & 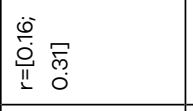 & 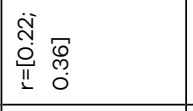 & 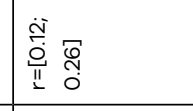 \\
\hline 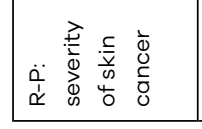 & 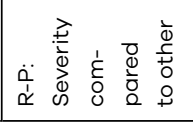 & 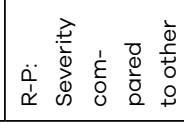 & 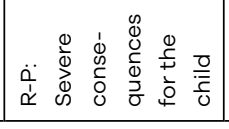 & 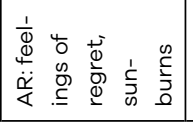 & 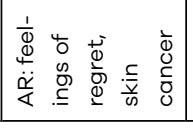 & 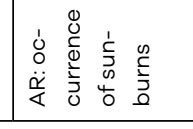 \\
\hline
\end{tabular}




\begin{tabular}{|c|c|c|c|}
\hline 荅 & 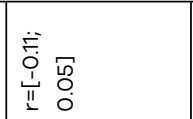 & 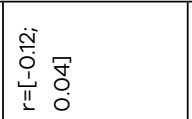 & 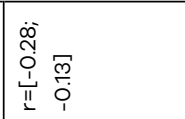 \\
\hline 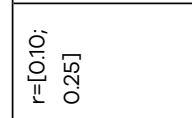 & 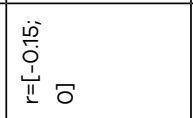 & 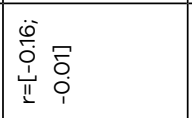 & în \\
\hline 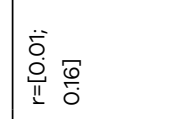 & 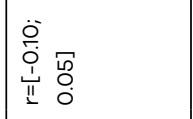 & \begin{tabular}{|ll}
0 & \\
0 \\
0 \\
1 & 0 \\
11 & 0 \\
11 & 0 \\
\end{tabular} & 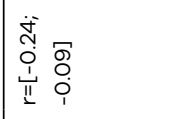 \\
\hline 范 & 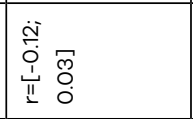 & 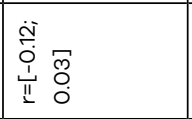 & 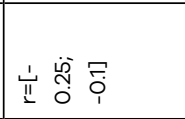 \\
\hline 总 & 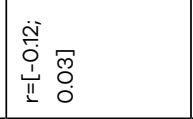 & 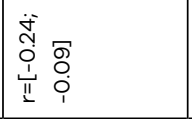 & 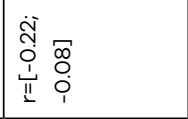 \\
\hline ". & 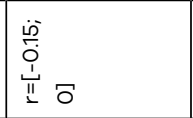 & 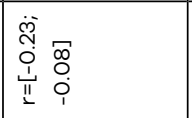 & 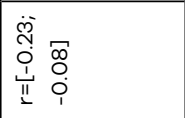 \\
\hline 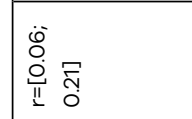 & 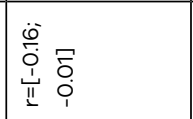 & 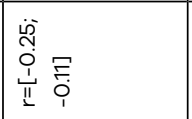 & 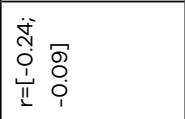 \\
\hline 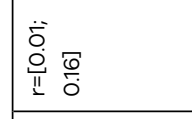 & 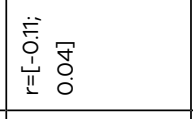 & 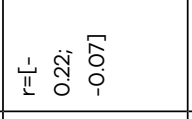 & $\begin{array}{lll} & \stackrel{0}{0} \\
& \stackrel{0}{0} \\
\end{array}$ \\
\hline 弚 & 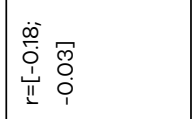 & 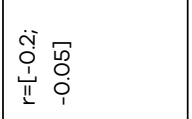 & 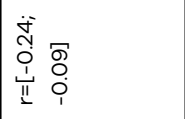 \\
\hline 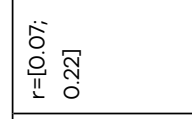 & 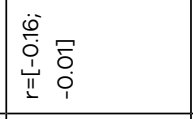 & 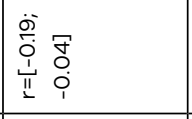 & 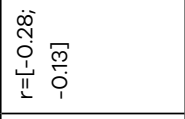 \\
\hline 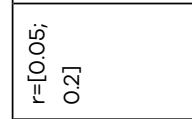 & 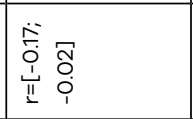 & 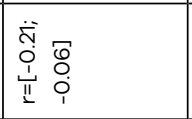 & 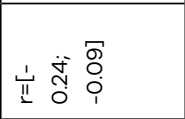 \\
\hline 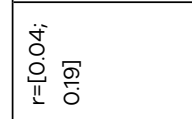 & 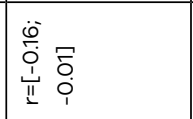 & 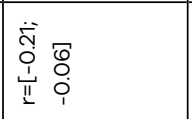 & 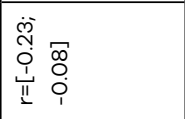 \\
\hline 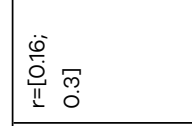 & 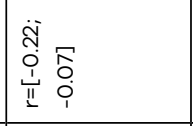 & 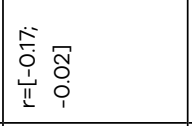 & 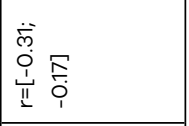 \\
\hline 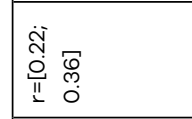 & 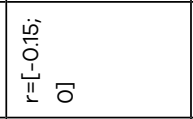 & 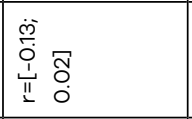 & 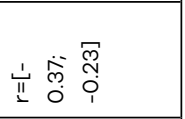 \\
\hline 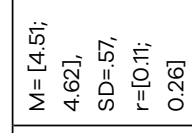 & 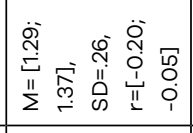 & 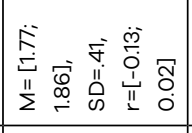 & 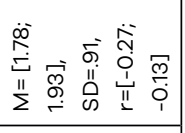 \\
\hline 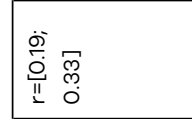 & 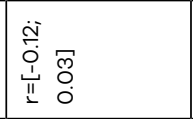 & 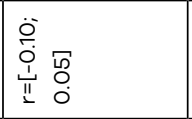 & נI \\
\hline 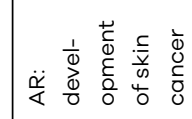 & 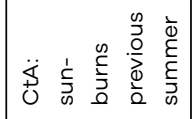 & 䒪 & 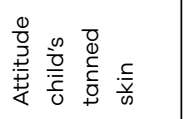 \\
\hline
\end{tabular}

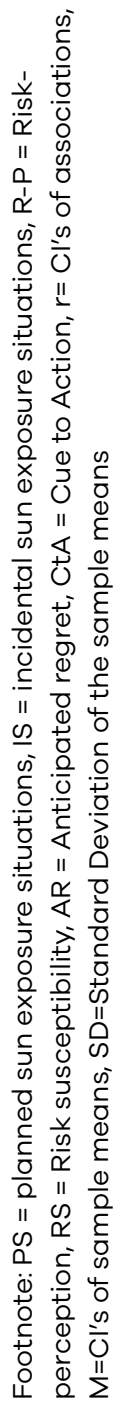


chapter four

\section{Childhood sun safety at different ages: relations between parental sun protection behaviour towards their child and children's own sun protection behaviour}


'I am always doing what I cannot do yet, in order to learn how to do it.'

_ Vincent van Gogh 


\section{Background}

Sunburns during childhood are strongly associated with development of melanoma in later life. While parents play an important role in children's sun protection, insight in possible shifts in behavioural responsibility from parents towards their children and the possible effect of children's sex is important for targeting sun safety interventions throughout childhood and adolescence.

\section{Methods}

This cross-sectional survey study was conducted among a representative sample of Dutch parents ( $N=1053$ ) of children aged between 4 and 13 years old. Questionnaires measured both parental and children's own sun safety behaviour during planned (e.g. going to the beach) and incidental (e.g. cycling) sun exposure situations. Analyses of variance were used to test for age group differences and linear regression models were computed to detect behavioural shifts in executive behaviour.

\section{Results}

Parents applied all sun safety behaviours (i.e. sunscreen use, wearing UV-protective clothing and seeking shade) more often on younger children, except for supportive behaviour (facilitating children's own sun safety behaviour), which remained relatively stable over the years. Older children and girls were more likely to execute sun safety behaviours themselves. A behavioural shift was found in wearing UVprotective clothing during planned situations among 11 year old children. For other behaviours, shifts were predicted after the age of 13 .

\section{Conclusions}

Older children execute sun safety behaviours more often than younger children, although they still largely depend on their parents' protection. Specific attention for boys in the primary school years, and for both boys and girls in the years adjacent to adolescence is warranted in skin cancer prevention interventions. 


\section{Background}

The incidence of both melanoma and non-melanoma skin cancer types among fair-skinned populations is increasing rapidly worldwide $(149,250-252)$. Factors that have been associated with these increased incidence rates are all related to Ultraviolet Radiation (UVR) exposure. Increased sun exposure behaviours during leisure-time activities, the preference for wearing less covered clothing and increased exposure to tanning beds, influenced by changed attitudes in which people are more in favour of getting a tan and enjoying the sun are important factors related to increasing skin cancer types $(96,251)$. Moreover, sun exposure and sunburns during the first 10 to 15 years of life have proven to play an important role in the aetiology of all skin cancer types $(46,62)$ and especially melanoma $(42,153,253,254)$ since children's skin is more sensitive to UVR. Even though sun exposure seems to be distributed equally over a person's lifetime, prevention of excessive sun exposure and sunburns during childhood is ought to start during early childhood (255).

Children are largely dependent of their parental sun protection, which makes them a particular vulnerable group (77). Moreover, childhood is an important phase in which health behaviours such as sun protection should be established (256) to increase the likelihood of habitual behaviour in later life (71). To prevent children from getting sunburnt, various precautions can be taken, in which parents play an important role. Application of sunscreen, wearing UV-protective clothing (including a hat and sunglasses) and seeking shade are the most important and recommended sun safe behaviours $(50,96,195)$.

Although clear guidelines exist with regard to sun protection of children, the prevalence of sunburns is still high, with studies reporting 28 to $52 \%$ of children suffering one or multiple sunburns during the past 12 months $(50,154,257,258)$. Although children in general are at high risk of getting sunburnt, several subgroups need specific attention. Boys in general seem to be more exposed to UVR (92), use less sun safe measures $(247,259)$ and have more reported sunburns during childhood $(260,261)$ than girls. Moreover, older children, primarily from the age of 8 , are often less protected against UVR than younger children, and more sunburns are reported $(50,51,79,80,195,247)$. Possible explanations for the finding that children are less protected as they get older is that older children spend more unsupervised time outside the house and gain self-responsibility and independence, which makes parental influence on children's behaviour less probable $(50,71,220,262)$. Furthermore, positive sun-protective attitudes and behaviours of children seem to weaken when children reach adolescence (79). However, parental sun safe attitudes, beliefs and behaviour are considered of great influence, even when a child grows older $(220,263,264)$. For example, Behrens and colleagues (52) found that parental attitudes favouring a tanned skin accounted for an $85 \%$ increase in sunburn risk of children aged 13 and above, while this effect was not seen among children aged between 0 and 6 years old. Even though parental influences seemingly play a vital role in sun safety behaviour, specific insight in the occurrence of sun safety behaviour of children themselves as they grow older are lacking. Although alterations in children's behavioural responsibility seem to occur within other health behaviours such as medicine use, healthy food intake or tooth brushing (265-268), occurrence of possible behavioural shifts concerning sun safety behaviour have not yet been investigated.

The primary school age is an important period in which children start to adopt and develop self-responsibility and autonomy over their health behaviour, a process that is continued throughout adolescence and is thought to result in the formation of habitual behaviour $(269,270)$. During this phase children learn that the environment expects them to start controlling their own behaviour and that their individual freedom expands $(262,271)$.

Given the fact that sunburn incidence rates are high among older children, gaining insight in possible behavioural shifts from parental executive to children's own executive behaviour is imperative for targeting interventions towards specific age groups and developing tailored content. Furthermore, based on existing differences in UVR exposure and sunburn incidence between boys and girls, examining the role of sex in the occurrence of sun safe behaviours of both parents and children as the child grows older is of great importance and can contribute to developing tailored sun safety interventions. 
This study therefore aims to gain insight in executive sun safe behaviours of parents and their child and investigates: 1. the relations between children's age and sex and the occurrence of extensive sun safe behaviours of both parents towards their child and children themselves in the age range of 4 to 13 years; and 2. the relations between children's age and sex on the one hand and the differences in execution of these behaviours between parents and their child on the other hand (i.e. investigation of when and how a possible behavioural shift takes place).

\section{Methods}

\section{Participants and procedures}

A cross-sectional survey study was conducted among Dutch parents $(n=1053)$ of children aged between 4 to 13 years, in November 2016. The Dutch research organization TNS-KANTAR (http://www.tns-nipo.com/) invited a sample of the Dutch population $(n=1222)$, representative with regard to education and income of the parents and age of the child, to participate via e-mail. Parents were eligible for participation if they had one or more children within the age range of the study (4-12years). Participants were invited to fill in an online questionnaire about sun safety behaviours regarding their youngest child. Respondents received one reminder e-mail. In total, 1053 parents filled in the questionnaire (86\%). TNS-KANTAR works with a permanent panel of respondents, who receive small incentives in terms of vouchers for their participation in studies. The data for this study were collected in November 2016. This study was exempt from review from the medical ethical commission, since respondents were not subjected to procedures, activities or behavioural requests (224). Respondents were not part of a vulnerable group. TNS-KANTAR retrieved online informed consent of all respondents beforehand. Since respondents were part of a survey panel, informed consent had to be given by definition (227). In accordance with the European Union-wide law on data protection (General Data Protection Regulation), the data in this study was not identifiable nor translatable to the respondents (225).

\section{Behavioural measures}

The online questionnaire contained demographic questions concerning, among others, sex and age of the child. Additionally, the frequency of execution of three sun safe behaviours during the previous summer season was asked about, for both the parent towards the child and the child him/herself. Sun safe behaviours consisted of sunscreen use, wearing UV-protective clothing, and seeking shade. Behavioural measures were based on Dutch guidelines regarding sun safety (73) and a previously validated questionnaire (272). Questions about children's executive behaviour were based on parental perceptions regarding their child's performance. Furthermore, supportive behaviour of parents was also asked for, which consisted of advising their child about sun safety, facilitating sun safety measures and checking whether the child applied sun safety behaviours. Explanatory text for all sun safe behaviours was used according to guidelines from the Dutch Cancer Society. A full overview of the outcome variables is provided in Table 1. 
Table 1. Sun safety behaviours of both parents and the child himself or herself

\begin{tabular}{|c|c|c|c|}
\hline & Behaviours & $\mathbf{N}$ of items & Exemplary item \\
\hline \multirow[t]{2}{*}{$\begin{array}{l}\text { Executive } \\
\text { behaviours: } \\
\text { 1. Sunscreen use }\end{array}$} & $\begin{array}{l}\text { Parents towards their child } \\
\text { Primary behaviour } \\
\text { - Sunscreen application in } \\
\text { general } \\
\text { Sub-behaviours } \\
\text { - Using a minimum of SPF } 30 \\
\text { - Applying sunscreen at least } \\
30 \text { minutes before sun exposure } \\
\text { - Reapplying sunscreen every } \\
\text { two hours }\end{array}$ & $\begin{array}{l}\text { ( } 8 \text { items; } 4 \text { in planned } \\
\text { situations, } 4 \text { in } \\
\text { incidental situations) }\end{array}$ & $\begin{array}{l}\text { 'To what extent did you } \\
\text { make sure your child was } \\
\text { sufficiently protected } \\
\text { with sunscreen when he/ } \\
\text { she was at the beach } \\
\text { or swimming pool/ } \\
\text { engaged in other outdoor } \\
\text { activities?' } \\
\text { (1. Never-5. Always) }\end{array}$ \\
\hline & $\begin{array}{l}\text { Parental perception of child's } \\
\text { behaviour } \\
\text { - Applying sufficient sunscreen }\end{array}$ & $\begin{array}{l}\text { ( } 2 \text { items; } 1 \text { in planned } \\
\text { situations, } 1 \text { in } \\
\text { incidental situations) }\end{array}$ & $\begin{array}{l}\text { 'My child applies } \\
\text { sunscreen sufficiently } \\
\text { when he/she goes to the } \\
\text { beach or swimming pool/is } \\
\text { engaging in other outdoor } \\
\text { activities.' } \\
\text { (1. Never - 5. Always, 6. I } \\
\text { don't know) }\end{array}$ \\
\hline \multirow[t]{2}{*}{$\begin{array}{l}\text { 2. Wearing } \\
\text { UV-protective } \\
\text { clothing }\end{array}$} & $\begin{array}{l}\text { Parents towards their child } \\
\text { Primary behaviour } \\
\text { - Wearing UV-protective } \\
\text { clothing } \\
\text { Sub-behaviours } \\
\text { - Wearing a long-sleeved t-shirt } \\
\text { - Wearing a cap or hat } \\
\text { - Wearing sunglasses }\end{array}$ & $\begin{array}{l}\text { (8 items; } 4 \text { in planned } \\
\text { situations, } 4 \text { in } \\
\text { incidental situations) }\end{array}$ & $\begin{array}{l}\text { 'To what extent did you } \\
\text { make sure your child was } \\
\text { wearing UV-protective } \\
\text { clothing when he/she was } \\
\text { at the beach or swimming } \\
\text { pool/engaged in other } \\
\text { outdoor activities?' } \\
\text { (1. Never - 5. Always) }\end{array}$ \\
\hline & $\begin{array}{l}\text { Parental perception of child's } \\
\text { behaviour } \\
\text { - Wearing UV protective } \\
\text { clothing }\end{array}$ & $\begin{array}{l}\text { ( } 2 \text { items; } 1 \text { in planned } \\
\text { situations, } 1 \text { in } \\
\text { incidental situations) }\end{array}$ & $\begin{array}{l}\text { 'My child puts on UV- } \\
\text { protective clothing when } \\
\text { he/she goes to the beach } \\
\text { or swimming pool/is } \\
\text { engaging in other outdoor } \\
\text { activities.' } \\
\text { (1. Never - 5. Always, } 6.1 \\
\text { don't know) }\end{array}$ \\
\hline $\begin{array}{l}\text { 3. Seeking } \\
\text { shade }\end{array}$ & $\begin{array}{l}\text { Parents towards their child } \\
\text { - Staying in the shade between } \\
12 \text { and } 3 \text { PM }\end{array}$ & $\begin{array}{l}\text { ( } 2 \text { items; } 1 \text { in planned } \\
\text { situations, } 1 \text { in } \\
\text { incidental situations) }\end{array}$ & $\begin{array}{l}\text { 'To what extent did you } \\
\text { make sure your child was } \\
\text { in the shade between } 12 \\
\text { and } 3 \text { PM when he/she was } \\
\text { at the beach or swimming } \\
\text { pool/engaged in other } \\
\text { outdoor activities?' } \\
\text { (1. Never - 5. Always) }\end{array}$ \\
\hline
\end{tabular}




\begin{tabular}{|l|l|l|l|}
\hline & $\begin{array}{l}\text { Parental perception of child's } \\
\text { behaviour } \\
\text { - Seeking shade during a sunny } \\
\text { day }\end{array}$ & $\begin{array}{l}\text { (2 items; } 1 \text { in planned } \\
\text { situations, } 1 \text { in } \\
\text { incidental situations) }\end{array}$ & $\begin{array}{l}\text { 'My child seeks shade } \\
\text { when he/she goes to the } \\
\text { beach or swimming pool/is } \\
\text { engaging in other outdoor } \\
\text { activities.' } \\
\text { (1. Never - 5. Always, 6. I } \\
\text { don't know) }\end{array}$ \\
\hline $\begin{array}{l}\text { Supportive } \\
\text { behaviour }\end{array}$ & $\begin{array}{l}\text { Parents towards their child } \\
\text { - Supporting the child's own } \\
\text { executive behaviour }\end{array}$ & $\begin{array}{l}\text { (2 items; 1 in planned } \\
\text { situations, 1 in } \\
\text { incidental situations) }\end{array}$ & $\begin{array}{l}\text { 'To what extent did you } \\
\text { support your child to } \\
\text { make sure he/she could } \\
\text { protect himself or herself } \\
\text { sufficiently?' } \\
\text { (1. Never - 5. Always) }\end{array}$ \\
\hline
\end{tabular}

\section{Sun exposure situations}

Both the three executive sun safety behaviours of both parents and their child, and supportive behaviour of the parents, were assessed in planned (e.g. going to the beach or the swimming pool) and incidental (e.g. being outside for other recreational purposes such as playing, cycling or walking) sun exposure situations (see Table 1).

\section{Statistical analyses}

Descriptive statistics were calculated for sex (\%) and age (M; SD) of the child and performance of sun safety behaviours of both parents and their child (M; SD). The sub-behaviour items within parental sunscreen use and putting on UV-protective clothing were not used in the analyses considering the fact that these behaviours are distinctive and therefore were not suitable for grouping. Missing values were coded when parents were unaware of the executive behaviour of their child. For the first research question, age of the child was categorized according to the Dutch primary school system. The youngest age group consisted of children between 4 and 6 years old (elementary school, grade 1 to 3 ), intermediate-aged children were between 7 and 9 years old (middle school, grade 4 to 6 ) and the oldest children were aged between 10 and 13 years old (senior school, grade 7 to 8 ).

For the first research question, two-way analyses of variance (ANOVA) were first performed to test for effects of interaction between age and sex of the child on the sun safety behaviours. When the null hypothesis of no interaction was rejected $(p<.05)$, one-way analyses of variance (ANOVA) were performed for boys and girls separately. Post hoc Tukey HSD tests were then used to test for differences between age groups. When the null hypothesis of no interaction was accepted, two-way analyses of variance were again performed after eliminating the interaction term. Sidak post hoc comparisons where then used for comparisons of the age groups $(p<.05)$. Possible sex differences in sun safety behaviours were then also investigated using the twoway ANOVA without the interaction term $(p<.05)$.

To examine the second research question relating to the development of the difference in sun safety behaviours of parents towards their child and children themselves, linear regression analyses were conducted. Difference scores involving mean scores of sunscreen use, wearing UV-protective clothing and seeking shade of the parents minus the mean scores of the child were calculated to test whether this difference between parental and child's behaviour decreases linearly as the child's age increases. The difference scores were computed for both planned and incidental sun exposure situations. To test for linearity, nine dummy variables for age, with the youngest age of 4 years as reference category, were formed for all remaining ages. Linear age models were then compared with saturated age models in which the nine 
dummy variables for age were put into the model. Linear age models were accepted when a significance value of $p \leq .05$ was reached. By extrapolating the regression results, possible behavioural shifts at later ages were predicted. To test for possible sex differences in the development of difference scores across age of the child, linear regression analyses were again performed, but then with the interaction between age and sex as additional predictor. Statistical analyses were performed using IBM SPSS Statistics for Windows, Version 21.0 (IBM Corp., 2013).

\section{Results}

\section{Sample characteristics and executive behaviours of parents and their child}

All parents ( $N=1053$ ) answered the questions, for 542 boys $(51.5 \%)$ and 510 girls $(48.5 \%)$. The children's mean age was 7.88 years $(S D=2.59)$. Of the three sun protection behaviours, sunscreen use was the most preferred method in both planned and incidental sun exposure situations by both parents and their child. Among the children, almost all behaviours were more frequently executed by girls than boys. Furthermore, supportive behaviour was also frequently applied by parents in both situations (see Table 2).

\section{Relations between child's age, sex and sunscreen use}

The relation of age and sex of the child on the one hand and parental sunscreen use on the other was examined for both planned and incidental sun exposure situations. An interaction between age and sex was found for both planned $(F(2,1095)=4.309, p=.014)$ and incidental $(F(2,1095)=3.516, p=.030)$ situations Parents applied sunscreen more frequently on youngest and intermediate-aged boys compared to older boys in both situations. Subsequently, parents applied sunscreen more often on younger aged girls than older ones, but only during incidental situations (Table 2).

There was no interaction between age and sex as regards the child's own sunscreen use. Differences between the three age groups however occurred in both planned $(F(2,1087)=89.300, p<.001)$ and incidental $(F(2,1091)=55.621, p<.001)$ situations. In both sun exposure situations, older children applied sunscreen more often than the youngest and intermediate-aged children, and intermediate-aged children performed sunscreen use more often than the youngest children. A full overview of results is provided in Table 2. Moreover, girls more frequently applied sunscreen than boys in both planned $(F(1,1087)=36.218, p<.001)$ and incidental $(F(1,1091)=27.351, p<.001)$ situations. 


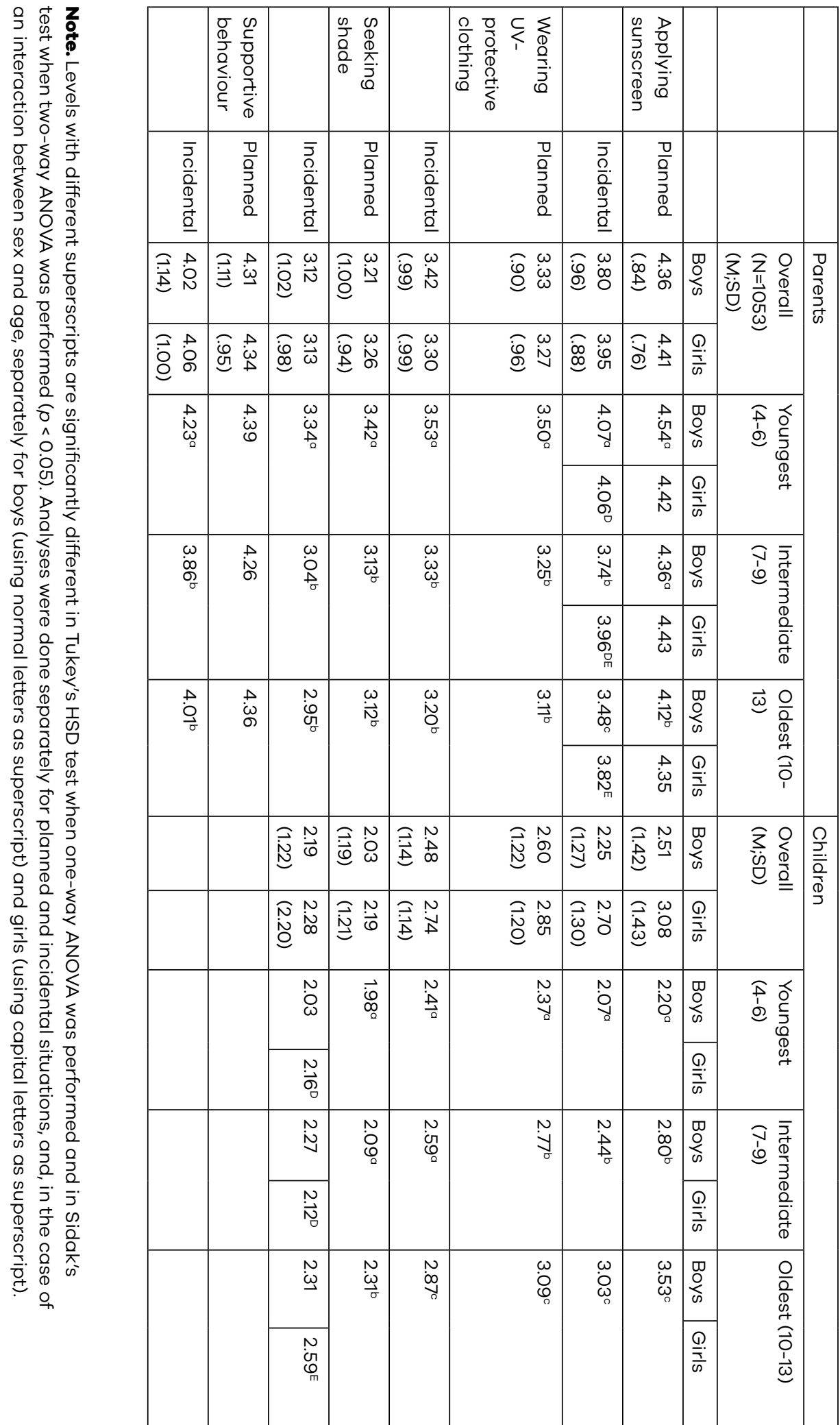




\section{Relations between child's age, sex and difference in child's and parental sunscreen use}

After performing regression analyses, the assumption of linear age effects was accepted for the relationship between the difference in sunscreen use of parents and their child with the age of the child, in both planned $(\beta=-.26, t(-14.25), p<.001)$ and incidental $(\beta=-.22, t(-13.46), p<.001)$ situations. This analysis shows that difference in sunscreen use between parents and their child decreases for older children, which might be caused by decreased application of sunscreen by parents and increased sunscreen use by children themselves as they grow older (see Fig. 1 depicting the trend across the child's age of both parental and children's sunscreen use at planned and incidental sun exposure situations). Across the age range studied, no shifts in sunscreen use, indicated by the child taking primary responsibility for sunscreen use (i.e. by applying sunscreen more often than the parents), were observed in either planned or incidental situations. However, based on extrapolation of the regression results, a shift in sunscreen use can be predicted approximately at the age of 14 years.

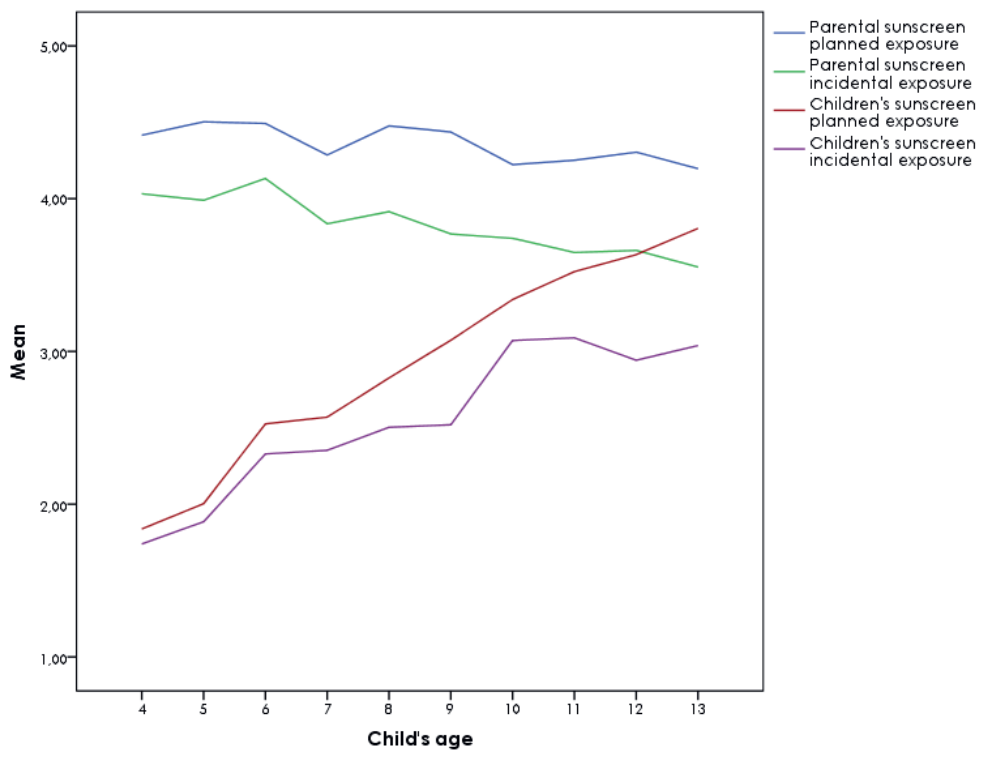

\section{Figure 1.}

Sunscreen use of parents towards their children and sunscreen use of the children themselves during planned and incidental sun exposure situations.

\section{Relations between child's age, sex and clothing behaviour}

No interaction of age and sex on the application of UV-protective clothing of parents towards their child was found. After eliminating the interaction term, however, age group differences were found in both planned $(F(2,1097)=15.195, p<.001)$ and incidental $(F(2,1097)=10.112, p<.001)$ situations. Parents put on UV-protective clothing more often on the youngest compared to intermediate-aged and oldest children in both situations (Table 2). Parents did not differ in putting on UV-protective clothing for boys and girls.

Again, no interaction between age and sex as regards child's own clothing behaviour was found. However, age group differences were also found in both planned $(F(2,1067)=46.394, p<.001)$ and incidental $(F(2$, 
$1082)=15.811, p<.001)$ situations. In planned situations, older children wore UV-protective clothes more often than all younger aged children, and intermediate-aged children executed the behaviour more often than the youngest children. In incidental situations, older children performed clothing behaviour most often compared to all younger children. See Table 2 for more detailed results. Moreover, girls put on protective clothing more often than boys in both planned $(F(1,1067)=9.380, p=.002)$ and incidental $(F(1,1082)=12.137$, $p=.001$ ) situations.

\section{Relations between child's age, sex and difference in child's and parental clothing behaviour}

The assumption of linear age effects was also accepted for the relationship between differences in wearing UV-protective clothing of parents and their child with the age of the child (see Fig. 2). In planned situations $(\beta=-18, t(-12.18), p=.000)$, we detected a behavioural shift at the age of 11 , after which the child executes clothing behaviour more often than the parents. In incidental situations ( $\beta=-.14, t(-9.46), p<.001)$, a shift is predicted by extrapolating the regression results, approximately at the age of 14 years.

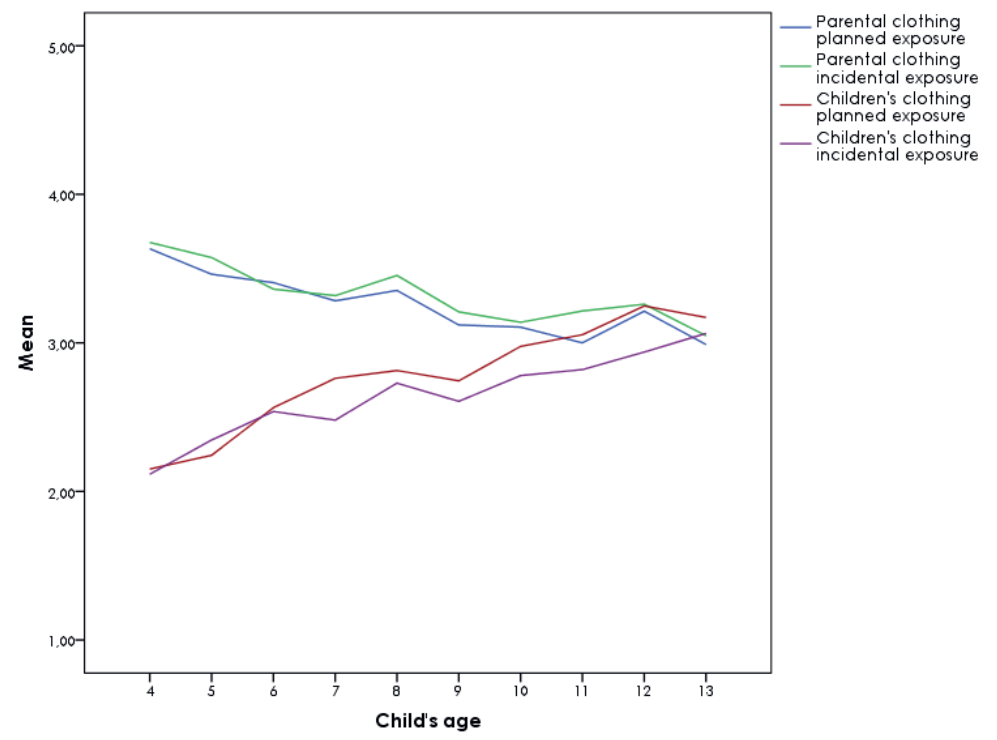

\section{Figure 2.}

Putting on UV-protective clothing of parents to their child and wearing UV-protective clothing of the child himself or herself in planned and incidental sun exposure situations.

\section{Relations between child's age, sex and seeking shade}

No interaction effects between age and sex were found for parental shade seeking. Age group differences concerning seeking shade were found in both planned $(F(2,1097)=11.284, p<.001)$ and incidental $(F$ $(2,1097)=15.907, p<.001)$ situations; parents keep their younger children more often in the shade than intermediate aged and oldest children. There were no differences observed in seeking shade for girls or boys. 
For children themselves, an interaction between age and sex was found only for seeking shade in incidental situations $(F(2,1087)=3.012, p=.05)$. Older girls sought shade more often than younger and intermediateaged girls. Furthermore, age group differences were found in planned situations $(F(2,1083)=7.211, p=.001)$, in which older children sought shade more often than all younger aged children (see Table 2 for details).

\section{Relations between child's age, sex and difference in child's and parental shade-seeking behaviour}

There was again a linear relation of age with the difference in shade-seeking behaviour in both planned $(\beta=-.09, t(-5.5), p<.001)$ and incidental $(B=-.12, t(-8.0), p<.001)$ situations (see Fig. 3$)$, suggesting that the difference in shade-seeking behaviour between parents and their child might diminish as the child grows older. However, before the age of 13 , children did not appear to seek shade more often than their parents. No shift in this behaviour is predicted until the age of 14 , meaning that the difference in shade-seeking behaviour of parents and children decreases slowly.

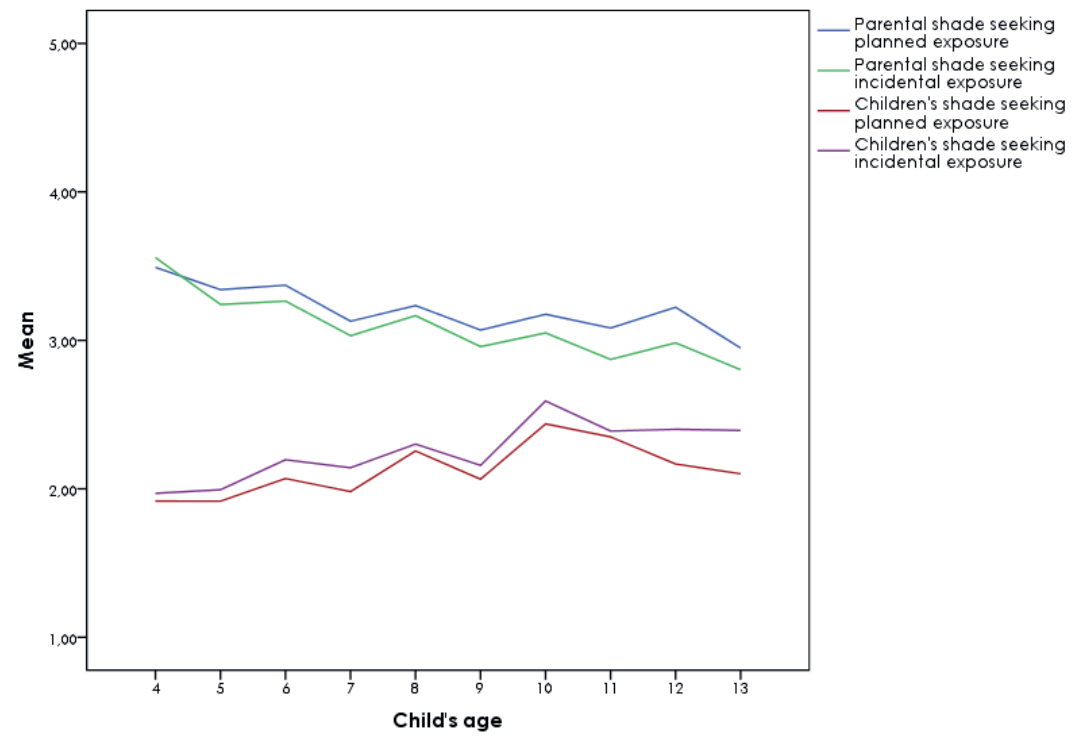

\section{Figure 3.}

Parents seeking shade for their child and shade-seeking behaviour of the child himself or herself in planned and incidental sun exposure situations.

\section{Sex and the relation of age with differences in sun safety behaviours between parents and their child}

For all sun safety behaviours, no significant differences between boys and girls concerning the relation between age and the difference in sun safety behaviours between parents and their child were observed. This indicates that children's sex does not play a role in the prediction of the difference between execution of sun safe behaviour of parents and their child as the child grows older. 


\section{Supportive behaviour of parents}

Finally, no interaction between age and sex was present for supportive behaviour of parents in both situations. Differences were observed between the three age groups $(F(2,1097)=10.553, p<.001)$ only in incidental sun exposure situations, which showed that parents more frequently perform supportive behaviour among the youngest compared to all older children. See Table 2 for detailed results. Furthermore, parents did not apply supportive behaviour more often among boys or girls.

\section{Discussion}

This study examined the differences between parents and their child concerning the execution of sun safe behaviours in the context of an increasing age of the child. Furthermore, effects of sex and age of the child on the development of these behaviours were investigated. When comparing sun safety behaviours, for both parents and children sunscreen use appeared the most frequently applied, followed by wearing UVprotective clothing and seeking shade, which corresponds with previous studies $(75,117,120)$. In addition, parents with young children applied sun safety measures significantly more often than parents with older children, which also corresponds with earlier work $(195,247)$. Additionally, older children were more likely to execute sun safe behaviours themselves. Interestingly, these results contrast with previous work, which state that sun protection of children themselves declines as children grow older $(77,79,247)$. Weakened parental encouragement towards the children as they grew older was mentioned as an important cause for this decline. In our study, supportive behaviour of parents remained relatively stable throughout the age range studied, which could have stimulated older children's sense of their own behavioural responsibility.

Furthermore, a possible effect of children's sex on sun safety behaviours was examined. Parents seem to apply sunscreen less frequently on older boys than younger ones in both planned and incidental situations, while age group differences for girls were only observed in incidental situations. Moreover, girls themselves executed all sun safety behaviours more often than boys in both situations. For shade-seeking behaviour, older girls more often stayed in the shade than younger girls, which is interesting since literature concerning sun safety behaviour among adolescents reveals that especially girls gain a desire to tan and are more likely to use tanning beds than boys, occurring around the age of 13 to 16 (120, 257, 273). Based on these results, stimulating sun safety behaviour for boys during early childhood deserves specific attention.

The overall results concerning the development of parental and child's sun safe behaviour indicate that during the age of 4 to 13 years, children increasingly apply sun safety behaviours, but largely depend on their parents' protection. The fact that children's sun safety is strongly related to adequate parental sun safety practices during early childhood is in line with previous studies $(80,195)$. Additionally, we found a shift in which children put on UV-protective clothing more often than parents do for them before the age of 13 in planned sun exposure situations. Shifts in other sun safety behaviours were subsequently mostly predicted after the age of 13 , when children transition into adolescence (274). Intervening on enhancing sun safety behaviours during this stage and some time before may be imperative for establishing sun safety behaviours in later life. This is accentuated by the fact that, at the onset of adolescence, increased levels of self-consciousness and internalization of norms and values develop (275), children start to form their own personal identity, and start to differentiate from their families (276). Moreover, before children reach adolescence, they are still prone to adopting their parents' values, which makes this specific age even more important to take into account (52). Nonetheless, children in the age of 4 to 12 years frequently get sunburnt, spend more time outdoors than adolescents and, from the age of 8, gain understanding in the influence they have on their own behaviour $(256,262)$, which makes them an important target group as well. Additionally, from a behavioural development perspective, it is important that children learn how to execute sun protective measures at an early age, rather than get familiar with these behaviours in later life, since unhealthy habits then may already have been established $(71,155)$. During the primary school years, stimulating an internal locus of control concerning health behaviours can enhance self-responsibility $(277,278)$. Sun safety interventions should therefore target both parents and children during the primary 
school age with a specific focus on boys. Parental influence is significant and life-long habits start to form, and children start gaining insight in their own health behaviours and can therefore be made aware about importance of sun safety. Additionally, when children transition into adolescence, interventions are of equal relevance, with a strong emphasis on self-responsibility.

There are a few limitations to this study that should be mentioned. First, children's own sun safety behaviour in this study consisted of parental perceptions of their execution, which may lower the validity of the outcome measure, even though parental perceptions of executive behaviours of their children is the most commonly used method in measuring sun safety behaviour (79). Moreover, frequency of sun safe behaviours was measured using retrospective self-report questions, which may limit the accuracy of behavioural outcomes because of social desirability and the possibility of recall bias $(248,263)$. To enhance objectification of sun safety behaviours, future studies should include personal dosimetry measures, preferably together with behavioural diaries (279). Moreover, combining objective measures with self-reported data allows for detecting risk situations regarding UVR exposure. Nevertheless, self-report measures remain a commonly used method for sun safety behaviours, with correlations among actual sun safe behaviours ranging from low to moderately positive $(279,280)$. A further limitation is that children's age in this study was limited to 13 years, while important behavioural shifts seemed to occur after this age. Even though statistical analyses allowed for prediction of these shifts, extrapolation based on cross-sectional data is less valid compared to time-series data. For future research, a within-subjects design using longitudinal data is essential to investigate behavioural changes of both parents and their child over time. Since findings about sun protection behaviour are weather dependent (272) and questions were asked regarding the past summer season, future studies are necessary to allow for seasonality. Additionally, elaborate data about objective UVR exposure of both parents and children in the Netherlands over time, is needed to target childhood sun safety interventions more accurately. Moreover, since children can be influenced by their caretaker's health behaviours and tend to imitate what they see $(51,77,281)$, future studies might also include questions regarding parental modelling and its effects on children's own executive behaviour.

The findings in this study concerning sun safe behaviours of children suggest that parental behaviour declines whereas children's own executive behaviour increases as a child grows older. Moreover, girls seem to protect themselves better than boys and parents apply sunscreen less on older boys than younger ones. However, children are not yet taking main responsibility for their sun safe behaviour during the assessed time interval (4-13 years), given the fact that a behavioural shift was only apparent in wearing UV-protective clothing during planned situations. These results lead to the recommendation that sun safety interventions during the primary school years should be focusing on both parents and their children, in which specific stimulation of sun protection among boys is warranted. In addition, since we predicted behavioural shifts after the age of 13, the age from which children reach adolescence suggests that this is an important target group as well for emphasizing that children take self-responsibility by providing them with advice and suggestions. However, follow-up data is imperative to consolidate the findings from this study and examine possible short-term developments.

\section{Conclusions}

In conclusion, the results from this study emphasize the importance of targeting sun safety interventions on both parents and their children during the primary school phase. Moreover, this study confirms that primary school-aged boys are an important target group for sun safety interventions as they are less protected than girls, while also highlighting the relevance of targeting interventions around the pre-adolescence phase for both boys and girls. Follow-up data on sun protection behaviour is however recommended to allow for weather dependent behaviour and to confirm these findings. 
chapter five

\section{Children's sunburn exposed: Identification of sun exposure and parental sun protection patterns}


'Life can only be understood backwards.

But it must be lived forwards.'

__ Søren Kierkegaard 


\section{Background}

Preventing sunburn in childhood is imperative in the light of skin cancer prevention. To provide directions for targeted interventions, a better understanding of children's sunburn and associated parental behaviours is necessary.

\section{Objective}

To explore sun exposure and parent-for-child sun protection patterns and their relation with experienced sunburn in children.

\section{Materials \& Methods}

An online survey study was conducted among parents ( $n=1299)$ of children (4 to 12 years). Latent Class Analysis (LCA) was performed to identify parental subgroups in children's sunburn, sun exposure and several sun protection behaviours (i.e. applying sunscreen, clothing, seeking shade) in two sun exposure settings (i.e. planned versus incidental). LCA results were validated by assessing predictions of class membership through several socio-demographic characteristics.

\section{Results}

Reported sunburn in the previous year was frequent $(>40 \%)$. Four latent classes of sunburn-exposure-protection were identified. Overall, the majority of parents reported fair sun protection behaviours. While lower protection behaviours were not strongly reflected in lower sunburn rates among the classes, high amounts of planned exposure (e.g. going to the beach) seemed to correspond with higher sunburn risk. Parents of younger children and those with more sensitive skin reported sun protection measures more frequently. Older children and those with more sensitive skin experienced more sunburn.

\section{Conclusions}

This study contributes to current insight in children's sunburn, based on parentproxy reports. Although a clear differentiation in sunburn risk was not found, several groups relevant for future interventions were indicated. By revealing the complexity of children's sunburn, suggestions for further research are provided. 


\section{Introduction}

Melanoma and non-melanoma skin cancers (NMSC) are some of the most rapidly increasing malignancies worldwide, affecting especially fair-skinned populations (4). Since the late 1980s, skin cancer incidence has tripled in the United States (20) and nearly quadrupled in the Netherlands (11, 27). Age-standardized incidence rates were estimated at 12.7 for the United States, compared to 25.7 for the Netherlands, 27.6 for Denmark, and 33.6 for Australia per 100,000 cases respectively in 2018 (22). Globally, melanoma represents the most fatal form of skin cancer and its incidence has risen more rapidly than any other malignancy in the last 50 years (20).

Overexposure to Ultraviolet radiation (UVR) and sunburn are major risk factors for developing melanoma, particularly when acquired during childhood (59). A meta-analysis revealed that having experienced sunburn at least once during childhood almost doubles the risk of developing melanoma (282). Additionally, although UVR-exposure throughout life increases melanoma risk, children's skin is more susceptible to damage, and the harmful effects can latently persist until adulthood (59). Children's skin has lower levels of protective melanin, resulting in a deeper penetration of UVR and greater damage to the skin (47). Limiting the amount of UVR and reducing sunburn incidence during childhood is therefore especially important. However, sunburn prevalence among children is still high, with reported percentages of children having experienced sunburn at least once during the previous year ranging between $29 \%$ to over $60 \%$ in the Netherlands, United States, Denmark and Switzerland $(48,50,52,55)$. Factors associated with increased sunburn risk among children, such as older age, sun-sensitive skin $(50,189)$, and past sunburn of parents or children $(51,52,189)$ were previously reported.

Preventing sunburn among children is of utmost importance. To protect the skin and prevent sunburn in children, several precautions can be taken, such as seeking shade, wearing UV- protective clothing and applying sunscreen. Performing these behaviours simultaneously is recommended, and should start in early life (6). Sunscreen undoubtedly remains the measure applied most frequently $(77,79,156)$ and is often used as sole prevention method among parents $(195,283)$. Seeking shade and wearing protective clothing are also considered highly important in order to enhance sun safety (194), but sunscreen is still advised to be applied as an adjunct to other forms of sun protection (6).

Multiple approaches are possible in order to promote children's sun safety. Targeting children directly via schools or day care centres show some promising results $(77,82,158)$. However, since parents and caregivers play a vital role in establishing children's sun safety and subsequent prevention of sunburn experiences, this indirect approach is considered very important as well $(51,71,77)$. Parents are primarily responsible for the application of sun protection measures for their children (79) and are most important in teaching children to perform their own sun protection behaviours (51). The large amount of influence of parents as active agents and role models is well reflected in strong correlations between parents' own sun protection behaviour and their children's sun safety $(52,80,203,247)$. Parents are therefore recommended to strictly adhere to sun protection guidelines and to teach their children adequate sun protection behaviours $(40$, 82), rendering them an essential target group for communications and interventions aimed at preventing children's sunburn.

Despite various studies reporting on parent-for-child sun protection behaviours, the association between the amount of children's UVR-exposure and performance of parent-for-child sun protection behaviours is underexplored. Equally unknown is how this exposure-protection connection affects children's sunburn experiences. Previous studies have focused on very young children (74, 119, 203), or did not report sun exposure patterns $(257,284)$. The link between sun exposure, sun protection behaviours and sunburn is regarded as complex and needs further clarification. Hence, in order to gain insight in children's sunburn, identification of risk-patterns of UVR-exposure and parent-for-child sun protection behaviours in various situations is necessary. 
The extent to which sun protection behaviours are necessary depends on the activities in which sun exposure is acquired. For example, when not actively focused on spending time outdoors, preparation efforts to protect ones' skin may be lower than when people intentionally expose themselves to the sun (285, 286). Consequently, the risk of sunburn increases in these unintentional sun exposure situations (e.g. playing outdoors) due to limited shade availability, prolonged duration of the exposure, or reliance on sunscreen use only, as shown by a recent study among adolescents and adults in New Zealand (287), and in Denmark (288). Two German studies among parents of very young children ( 3 to 6 years) revealed different parent-for-child sun protection behaviours: shade-seeking behaviour and wearing hats and sunglasses were applied more often in a beach setting than during everyday outdoor activities $(78,289)$. Moreover, parental sun protection behaviours among older children vary across sun exposure situations $(156,290)$, and their relationship with sunburn is largely undocumented. There is evidence that the more intense and intermittently received UVR, e.g. during recreational activities, the greater the melanoma risk $(59,153)$. Therefore, shedding light on the association of different types of sun exposure, sun protection strategies and their relation to children's sunburn is expected to generate a better understanding of sunburn risk in different exposure settings. This knowledge would facilitate the development and implementation of targeted policy and interventions to counteract the increasing melanoma incidence rates.

This study aimed to explore the heterogeneity of inter-relations between: (1) children's sun exposure; (2) parental sun protection behaviours; and (3) children's sunburn frequency. To achieve this goal, we applied latent class analysis (LCA), a model-based clustering technique (291), to empirically derive typologies capturing distinct patterns of inter-connections across the three domains. Further, links between the identified classes and parental and children's socio-demographic characteristics were investigated to explore possible subgroups that deserve specific attention in future interventions.

\section{Materials and methods}

\section{Procedure and participants}

This study was part of a longitudinal survey study whose data from the baseline measurement (June 2016 (TO)) was analysed. The study was exempt from medical ethical evaluation since participants were not exposed to medical procedures (224). The collected data was not traceable to specific participants in the dataset (225).

Eligible participants, caring for at least one child of primary school age ( 4 to 12 years), were retrieved from an existing research panel and received one invitational and one reminder email. A sample of 2449 parents, representative of the Dutch general population according to education level and income, were approached by research organisation TNS-KANTAR. Informed consent was acquired online (227). In total, 1299 Dutch parents filled in the online questionnaire. Parents obtained gift vouchers after completing the survey.

\section{Measurement}

The online questionnaire assessed, amongst others, 1) demographic characteristics; 2) children's sunburn experiences; 3 ) the amount of children's sun exposure; and 4) parental sun protection behaviours (i.e. sunscreen use, clothing, and seeking shade) in both planned and incidental sun exposure situations. Planned situations concerned sun exposure that is generally anticipated by the parent and/or the child (e.g. going to the beach or swimming pool), whereas incidental situations consisted of less intentional sun exposure situations (e.g. when walking, cycling or playing outdoors). Children's sunburn, sun exposure and sun protection behaviours during the previous summer season were assessed for the youngest child in the parents' household. The full questionnaire can be retrieved from Open Science Framework (OSF): https:// osf.io/zncwq/. This study is reported in accordance with STROBE guidelines for observational research (226). 


\section{Socio-demographic characteristics}

Parental and children's age, sex, skin type and parental educational level and province of residence were assessed. Parental age was categorized in three groups (18-34 years, $35-44$ years, and $\geq 45$ years), as was children's age (4 to 6 years, 7 to 9 years, and $\geq 10$ years), the latter in agreement with the Dutch primary school system. Age categories were referred to as young, middle-aged and older. Parental and children's skin type was categorized in: I) burns very quickly, tans never or rarely; II) burns quickly, tans slowly; III) burns rarely, tans easily; and IV) burns almost never, tans very easily. Educational level was classified in lower (1), intermediate (2) and higher (3) education, cf. guidelines of Statistics Netherlands (228). The twelve Dutch provinces of residence were subdivided into North (1), East (2), West (3) and South (4), cf. classification of Dutch Ministry of the Interior and Kingdom Relations (292).

\section{Children's sunburn}

The frequency of children's sunburn was assessed using one item, in which parents were asked how often their child had experienced sunburn in the past 12 months. A 5-point Likert scale was used (never (1), 1-2 times (2), 3-4 times (3), 4-5 times (4) and > 5 times (5)).

\section{Children's sun exposure}

The amount of received sun exposure was assessed by asking parents the frequency with which their child was outside on sunny days during the previous 12 months, using a 5-point Likert scale (never (1), rarely (2), sometimes (3), often (4), as often as possible (5)). The questions regarding sun exposure were asked using two items; one regarding planned situations and one for incidental situations. Planned sun exposure involved intentional sun-seeking behaviour (e.g. going to the beach or swimming pool) and incidental sun exposure consisted of all other situations in which children were sun-exposed (e.g. playing outdoors, cycling). Parents received an explanatory text regarding how to distinguish between these sun exposure situations.

\section{Parental sun protection behaviours}

Three parent-for-child sun protection behaviours were assessed:1) sunscreen use;2) clothing; and 3) seeking shade. The execution of these behaviours was asked about regarding the two (planned and incidental) sun exposure situations, resulting in six items. A 5-point Likert scale was again used to assess the frequency of sun protection behaviours (never (1), rarely (2), sometimes (3), often (4) and always (5)). Sufficient sunscreen application encompassed using an SPF $\geq 30$, applying sunscreen 30 minutes prior to sun exposure, and reapplying sunscreen every two hours. For clothing, wearing a shoulder-covering T-shirt, wearing a hat and wearing sunglasses was regarded as sufficient. Lastly, seeking shade between UV-peak hours, 12 and $3 \mathrm{PM}$, was considered adequate. Explanatory text regarding the sufficient performance of sun protection behaviours was provided in the questionnaire.

\section{Variables operationalisation}

Data preparation for statistical analyses took place by recoding the categorical variables. An inspection of the answers' frequency distribution showed very low count in some response categories. For this reason, all categorical variables were collapsed to avoid sparsity problems. Children's sunburn frequency was recoded as never (1), 1-2 times (2), $\geq 3$ times (3), while children's sun exposure in both planned and incidental situations was recoded as never (1), rarely and sometimes (2), often and as often as possible (3), and parental execution of sun protection behaviours in both sun exposure situations as never (1), rarely and sometimes (2), often and always (3). Based on this recoding, three answer categories were obtained across all items. First, the least protective answer category included the responses of $\geq 3$ sunburn experiences, highest reported levels of sun exposure and lowest reported levels of sun protection behaviours. Second, the intermediate answer category contained responses of 1-2 sunburn experiences, being rarely and sometimes sun-exposed, and having rarely and sometimes performed sun protection behaviours. Lastly, the most optimal answer category consisted of the responses of having never experienced sunburn, lowest reported level of sun exposure and highest levels of reported sun protection behaviours. 


\section{Statistical methods}

Descriptive statistics (means, standard deviations and percentages) were computed to examine the distribution of participants' characteristics, children's sunburn frequency, children's sun exposure and the frequency of parental sun protection behaviours. McNemar-Bowker and Chi-square tests were conducted to explore differences in sample protection behaviours in planned vs. incidental sun exposure situations and for sunburn frequency, respectively. Significance level was set at 5\%. IBM SPSS Statistics for Windows, version 25.0, was used (IBM Corp., 2017).

\section{Analysis plan}

\section{Latent Class Analysis - model fitting}

Latent Class Analysis (LCA), a model-based clustering technique, was conducted to explore the heterogeneity of children's sunburn and behavioural patterns (both children's sun exposure and parental sun protection behaviours) $(291,293)$. The non-directly observable data-driven classes were revealed by grouping participants with similar responses to items related to sunburn, sun exposure, and sun protection behaviours, but different from other groups. Class-enumeration, i.e. determining the number of latent classes, was guided by the model fit Akaike Information Criterion (AIC) and adjusted Bayesian Information Criterion (BIC) (294), while visually assessing whether extracted classes were qualitatively distinct from each other. After settling for the final number of classes, post-hoc posterior probabilities of class membership was computed for each subject (291), who was then assigned to the class with the highest posterior probability. The index of entropy, though not used to guide class-enumeration, was also reported to indicate the quality of classification uncertainty (entropy values above 0.80 are considered of very good classification performance). LCA was executed using SAS software version 9.4 (295).

\section{Classes' profiling}

Chi-square tests were performed to explore unadjusted differences among extracted classes in sociodemographic characteristics (parental age, sex, skin type, province of residence and educational level, and children's age, sex and skin type). A multinomial linear regression model was fitted to establish which of these characteristics remained independently linked to class membership after mutual adjustment. Estimated odds ratio (OR) and 95\% Confidence Intervals (Cls) are displayed in forest plots. To avoid multicollinearity in the multinomial logistic regression, age and skin type of the child rather than of the parents were included in the model.

\section{Results}

\section{Socio-demographic characteristics}

Of the included parents, 773 (59.5\%) were mothers and the questionnaire was answered for $656(50.5 \%)$ boys. Parents' age was normally distributed (range: 18 to 69 years) and most often between 40 and 44 years old (32.6\%). Children's age was not evenly distributed, resulting in children most often being between 4 and 6 years old ( $n=595 ; 45.8 \%)$. Most children had skin type III (burns rarely) $(49.7 \%)$, followed by skin type II (burns quickly) (35.9\%). Most parents received higher education ( $n=625 ; 48.3 \%$ ).

\section{Sunburn, sun exposure and sun protection behaviours}

Overall, during the previous year, 550 children (42.4\%) had experienced at least one case of sunburn. Older children $\left(X^{2}(4)=12.80, p=.012\right)$ and children with more sensitive skin $\left(X^{2}(6)=111.52, p<.001\right)$ experienced more sunburn. Parents of younger children and children with more sensitive skin reported more frequent execution of almost all sun protection behaviours than parents of younger children and with less sensitive skin types. Sunscreen use and seeking shade were more often performed in planned situations, and 
clothing in incidental situations. Table 1 provides an overall summary of the whole sample, and as such, only a general overview of the marginal distributions. A more refined glimpse into the data is provided by the LCA findings.

Table 1. Parent-for-child sun protection behaviours $(\mathrm{N}=1299)$

\begin{tabular}{|c|c|c|c|c|c|}
\hline \multicolumn{4}{|c|}{ Comparisons between planned and incidental exposure } & \multirow{2}{*}{\begin{tabular}{|l}
$\begin{array}{l}\text { McNemar- } \\
\text { Bowker test }\end{array}$ \\
$X^{2}(3)=330.91$ \\
\end{tabular}} & \multirow{2}{*}{\begin{tabular}{|l|} 
p-value \\
$<.001$ \\
\end{tabular}} \\
\hline Sunscreen & & Planned & Incidental & & \\
\hline & Never & $6(.5)$ & $16(1.2)$ & & \\
\hline & Rarely/Sometimes & $98(7.5)$ & $431(33.2)$ & & \\
\hline & Often/Always & $1195(92.0)$ & $852(56.6)$ & & \\
\hline \multirow[t]{4}{*}{ Clothing } & & Planned & Incidental & $X^{2}(3)=28.01$ & $<.001$ \\
\hline & Never & $23(1.8)$ & $24(1.8)$ & & \\
\hline & Rarely/Sometimes & $643(49.5)$ & $551(42.4)$ & & \\
\hline & Often/Always & $633(48.7)$ & $724(55.7)$ & & \\
\hline \multirow[t]{4}{*}{ Seeking shade } & & Planned & $\underline{\text { Incidental }}$ & $X^{2}(3)=18.32$ & $<.001$ \\
\hline & Never & $53(4.1)$ & $59(4.5)$ & & \\
\hline & Rarely/Sometimes & $731(56.3)$ & $782(60.2)$ & & \\
\hline & Often/Always & $515(39.6)$ & $458(35.3)$ & & \\
\hline \multicolumn{6}{|c|}{ Parent-for-child sun protection behaviours stratified by child's age and skin type (Chi-square) } \\
\hline & & Child's age & p-value & Child's skin type & p-value \\
\hline \multirow[t]{2}{*}{ Sunscreen } & Planned & $X^{2}(4)=6.57$ & .16 & $X^{2}(6)=16.55$ & .01 \\
\hline & Incidental & $X^{2}(4)=15.32$ & .004 & $X^{2}(6)=28.56$ & $<.001$ \\
\hline \multirow[t]{2}{*}{ Clothing } & Planned & $X^{2}(4)=13.21$ & .01 & $x^{2}(6)=36.35$ & $<.001$ \\
\hline & Incidental & $X^{2}(4)=26.66$ & $<.001$ & $X^{2}(6)=24.20$ & $<.001$ \\
\hline \multirow[t]{2}{*}{ Seeking shade } & Planned & $X^{2}(4)=17.72$ & .001 & $X^{2}(6)=11.18$ & .08 \\
\hline & Incidental & $x^{2}(4)=23.63$ & $<.001$ & $x^{2}(6)=16.12$ & .013 \\
\hline
\end{tabular}




\section{Identifying subgroups (Latent Class Analyses)}

Models with three to six latent classes were fitted. Table 2 shows model fit assessment criteria that were used to guide class-enumeration. They were not in full agreement, but visual inspection of the distinctiveness of the classes together with the BIC indicated that a solution with four classes was a good fit to the data.

Table 2. Model fit evaluation of Latent Classes

\begin{tabular}{|l|l|l|l|}
\hline Number of Latent Class & AIC & Adjusted BIC & Entropy \\
\hline 3 & 931.18 & 1042.78 & .88 \\
\hline $\mathbf{4}$ & $\mathbf{8 9 1 . 2 1}$ & $\mathbf{1 0 4 0 . 6 7}$ & $\mathbf{. 8 2}$ \\
\hline 5 & 862.12 & 1049.45 & .84 \\
\hline 6 & 845.25 & 1070.44 & .85 \\
\hline
\end{tabular}

Figure 1 illustrates the distinct response patterns to sunburn, and exposure and protective behaviours questions of the four extracted classes. The plot shows the probability of class members of reporting high-risk, intermediate-risk and low-risk answers to sunburn, sun exposure and sun protection items. For instance, the $y$-axis of subplot $1 \mathrm{~A}$ shows the probability for selecting the high-risk answer category, i.e. highest frequency of sunburn ( $\geq 3$ sunburn), highest reported sun exposure (often and as often as possible) and lowest reported levels of sun protection behaviours (never performed), while the $x$-axis indicates whether the situation was planned or incidental. Similarly, figures $1 \mathrm{~B}$ and $1 \mathrm{C}$ show the probabilities of intermediate- and low-risk answer patterns.

Sunburn probability was relatively high (between $\sim 40$ and $45 \%$ for 1 or 2 cases of sunburn; $50 \%$ of no sunburn) for three out of the four classes (1B), showing a low discriminatory power between these classes for this item. Class 1 demonstrated a lower sunburn risk ( $30 \%$ of cases of 1 or 2 sunburn; $70 \%$ of no sunburn). The probability of having children with $\geq 3$ reported sunburns was very low among all classes (1A).

Sun exposure patterns showed a larger discriminatory power between classes. Class 1 and 2 , which made up the majority of parents $(>70 \%)$ reported highest levels of exposure in incidental situations and the remaining classes ( 3 and 4 ) reported lower incidental exposure than planned exposure (1A). Of note, class 1 shows almost complete withholding of planned sun exposure.

Sun protection patterns are again distinct between the classes. Class 1 and 2 reported relatively high levels of sun protection behaviours in both situations, whereas the remaining two classes ( 3 and 4 ) showed more variability, with protective behaviours being more frequent in planned rather than incidental situations. The latter classes had a clear preference for sunscreen use, followed by clothing and seeking shade, respectively. Of note, these classes differed in degree of sun protection behaviours, with class 3 showing the least sun protection behaviours (Figure 1C). 

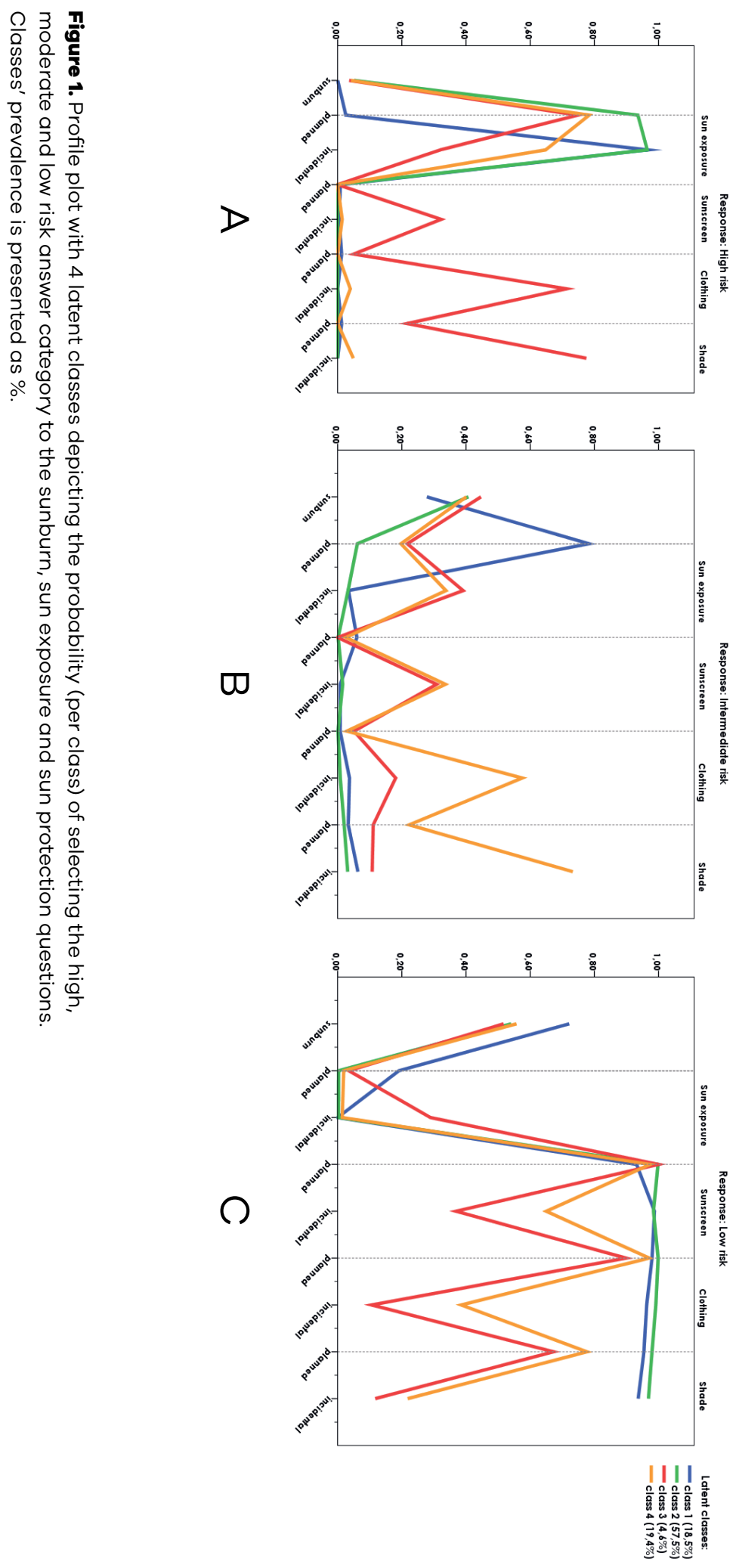
The four classes were named primarily reflecting parental sun protection behaviours:

1. Class 1 with adequate sun protection behaviour ( $n=288 ; 30.6 \% \geq 1$ sunburn) is considered the best performing class as reflected in: a) lowest sunburn probability; b) consistent reporting of high levels of sun protection behaviour; and c) no exposure in planned situations and high exposure in incidental situations.

2. Class 2 with adequate sun protection behaviour ( $n=726 ; 46.3 \% \geq 1$ sunburn), is defined as the second-best performing class as reflected in: a) moderate sunburn probability; b) consistent reporting of high levels of sun protection behaviour; and c) high levels of sun exposure in planned and incidental situations, indicating unfavourable exposure patterns.

3. Class 3 with inadequate sun protection behaviour ( $n=53 ; 51 \% \geq 1$ sunburn), is considered the poorest performing class as reflected in: a) moderate sunburn probability; b) low levels of sun protection behaviours in particular incidental situations; and c) lower levels of sun exposure in planned and especially incidental situations, indicating a more favourable exposure pattern.

4. Class 4 with inadequate sun protection behaviour ( $n=232 ; 42.7 \% \geq 1$ sunburn) is defined as the intermediate performing class as reflected in: a) moderate sunburn probability; b) lower levels of sun protection behaviours; and c) lower levels of sun exposure in planned and especially incidental situations.

\section{Socio-demographic class-profiling}

Next, we compared whether parents' and children's characteristics differed across the four classes. In general, the majority of parents were middle-aged, had quickly burning (II) or rarely burning (III) skin complexions, were highly educated and lived in the Western part of the Netherlands. The majority of children also had skin type II or III. Differences in both parental and children's age and skin type (Table 3) distributions were observed across classes, with the best and second-best class including younger children with more sensitive skin types.

Table 3. Socio-demographic characteristics and class-profiling

\begin{tabular}{|c|c|c|c|c|c|}
\hline & $\begin{array}{l}\text { Best class } \\
(n=288 ; 18.5 \%)\end{array}$ & $\begin{array}{l}\text { Second-best } \\
\text { class } \\
(n=726 ; 57.5 \%)\end{array}$ & $\begin{array}{l}\text { Poorest } \\
\text { class } \\
(n=53 ; 4.6 \%)\end{array}$ & $\begin{array}{l}\text { Intermediate class } \\
(n=232 ; 19.4 \%)\end{array}$ & \\
\hline & $n(\%)$ & $n(\%)$ & $n(\%)$ & $n(\%)$ & $\begin{array}{l}\text { p-value } \\
\text { (Chi- } \\
\text { Square) }\end{array}$ \\
\hline $\begin{array}{l}\text { Parents' } \\
\text { characteristics }\end{array}$ & & & & & \\
\hline Age & & & & & .003 \\
\hline - $\quad$ Young & $53(18.4)$ & $102(14.0)$ & $6(11.3)$ & $21(9.1)$ & \\
\hline - $\quad$ Middle-aged & $182(63.2)$ & $449(61.8)$ & $27(50.9)$ & $143(61.6)$ & \\
\hline - $\quad$ Older & $53(18.4)$ & $175(24.1)$ & $20(37.7)$ & $68(29.3)$ & \\
\hline Sex & & & & & .220 \\
\hline - Male & $110(38.2)$ & $286(39.4)$ & $26(49.1)$ & $104(44.8)$ & \\
\hline - Female & $178(61.8)$ & $440(60.6)$ & 27 (50.9) & $128(55.2)$ & \\
\hline
\end{tabular}




\begin{tabular}{|c|c|c|c|c|c|}
\hline Skin type & & & & & $<.001$ \\
\hline $\begin{array}{l}\text { - } \quad \text { I: burns very } \\
\text { quickly }\end{array}$ & $31(10.8)$ & $77(10.6)$ & $2(3.8)$ & 9 (3.9) & \\
\hline $\begin{array}{l}\text { - } \quad \text { Il: burns } \\
\text { quickly }\end{array}$ & $124(43.1)$ & $284(39.1)$ & $12(22.6)$ & $96(41.4)$ & \\
\hline $\begin{array}{l}\text { - III: burns } \\
\text { rarely }\end{array}$ & 114 (39.6) & $322(44.4)$ & $30(56.6)$ & $101(43.5)$ & \\
\hline $\begin{array}{ll}\text { - } & \text { IV: burns } \\
& \text { almost never }\end{array}$ & $19(6.6)$ & $43(5.9)$ & $9(17.0)$ & $26(11.2)$ & \\
\hline Education & & & & & .118 \\
\hline - Lower & $36(12.5)$ & $102(14.0)$ & $11(20.8)$ & $43(18.5)$ & \\
\hline - Intermediate & $111(38.5)$ & 278 (38.5) & 20 (37.7) & 69 (29.7) & \\
\hline - Higher & $141(49.0)$ & $343(47.2)$ & $21(39.6)$ & $120(51.7)$ & \\
\hline Province & & & & & .135 \\
\hline - North & $19(6.6)$ & 68 (9.4) & $2(3.8)$ & $19(8.2)$ & \\
\hline - $\quad$ East & $74(25.7)$ & $162(22.3)$ & $8(15.1)$ & $61(26.3)$ & \\
\hline - $\quad$ South & $132(45.8)$ & $308(42.4)$ & 33 (62.3) & $100(43.1)$ & \\
\hline - West & $63(21.9)$ & $187(25.8)$ & 10 (18.9) & $52(22.4)$ & \\
\hline \multicolumn{6}{|l|}{$\begin{array}{l}\text { Children's } \\
\text { characteristics }\end{array}$} \\
\hline Age & & & & & $<.001$ \\
\hline - $\quad$ Young & 172 (59.7) & $326(44.9)$ & 15 (28.3) & 82 (35.3) & \\
\hline - $\quad$ Middle-aged & $71(24.7)$ & $221(30.4)$ & $22(41.5)$ & $71(30.6)$ & \\
\hline - $\quad$ Older & $45(15.6)$ & $179(24.7)$ & $16(30.2)$ & 79 (34.1) & \\
\hline Sex & & & & & .945 \\
\hline - Boy & $145(50.3)$ & $366(50.4)$ & $25(47.2)$ & $120(51.7)$ & \\
\hline - Girl & $143(49.7)$ & 360 (49.6) & $28(52.8)$ & $112(48.3)$ & \\
\hline Skin type & & & & & .001 \\
\hline $\begin{array}{l}\text { - } \quad \text { I: burns very } \\
\text { quickly }\end{array}$ & 24 (8.3) & $51(7.0)$ & $1(1.9)$ & $5(2.2)$ & \\
\hline $\begin{array}{l}\text { - } \quad \text { Il: burns } \\
\text { quickly }\end{array}$ & 112 (38.9) & $264(36.4)$ & $14(26.4)$ & $76(32.8)$ & \\
\hline $\begin{array}{l}\text { - III: burns } \\
\text { rarely }\end{array}$ & $131(45.5)$ & $363(50.0)$ & $29(54.7)$ & 123 (53.0) & \\
\hline $\begin{array}{ll}\text { - } & \text { IV: burns } \\
& \text { almost never }\end{array}$ & $21(7.3)$ & $48(6.6)$ & $9(17.0)$ & $28(12.1)$ & \\
\hline
\end{tabular}

These differences were further confirmed after mutual adjustment (Figure 2). Results revealed that parents assigned to the best two classes had the youngest children with most sensitive skin complexions, while the best group (Class 1) included the youngest children. The two remaining classes included parents of older children, with less sensitive skin types. The total model was adjusted for parental age, sex, skin type, province of residence, educational level and children's age, sex and skin type. All model parameter estimates can be retrieved from Appendix I. 


\section{Second-best class}

Skin burns very quickly (I)

Skin burns quickly (II)

Skin burns rarely (III)

Youngest child

Middle-aged child

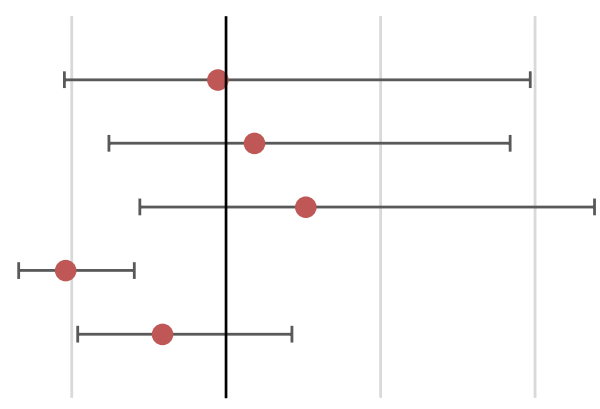

0

0,5

1,5

2

\section{Poorest class}

Skin burns very quickly (I)

Skin burns quickly (II)

Skin burns rarely (III)

Youngest child

Middle-aged child

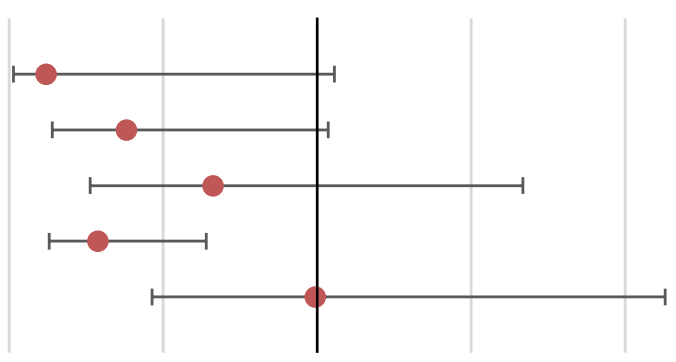
0
0,5
1,5
2

\section{Intermediate class}

Skin burns very quickly (I)

Skin burns quickly (II)

Skin burns rarely (III)

Youngest child

Middle-aged child

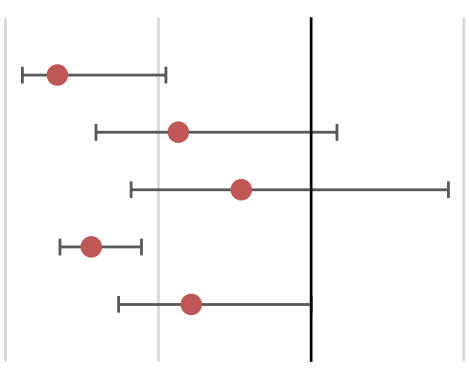

0

0,5

1,5

2

Figure 2. Forest plots with odds ratios (ORs) and associated Cl's (95\%) of statistically significant results of the multinomial (multivariable) logistic regression, demonstrating socio-demographic characteristics linked to class membership - OR are provided with respect to the best class (1). Reference group for skin type is 'Skin never burns (IV)'; Reference group for children's age is 'oldest child'. 


\section{Discussion}

This study exposed differences in parental sun protection, children's sun exposure patterns and children's sunburn. Overall, sunburn frequency was high $(>40 \%)$ among all children, and parents reported fair sun protection behaviours. Latent Class analysis revealed four sun (burn-exposure-protection) typologies, which, while differing in frequency and type of sun-protective strategies, could not be firmly distinguished in terms of sunburn frequency for three out of the four classes. Parents engaged in more protective measures during planned rather than incidental sun exposure. Avoiding sun exposure in these planned situations (e.g. going to the beach or swimming pool) seemed to be associated with a lower sunburn risk. These findings seem to confirm the complexity of the relationship between children's sunburn, sun exposure and parental sun protection behaviours: although the majority of parents were reporting relatively high levels of sun protection measures (Class 1 and 2), this resulted in a lower sunburn probability among only a small group of children (Class 1). This latter group included parents of young children and children with more sensitive skin types, who were inclined to limit sun exposure for their children in planned situations.

Substantial numbers of children in all four classes experienced at least one case of sunburn in the previous year, which is comparable to findings in other countries $(48,50,52)$. Parents in the best class with lowest yet notable sunburn prevalence $(>30 \%)$ were engaging in all sun protection behaviours while limiting their children's sun exposure in planned situations. They seemed to have been highly aware of the importance of sun protection behaviours and sun avoidance, but their children's sun exposure in incidental situations was still high. This may indicate, in line with previous research, that parents underestimate the risk of UVRexposure during everyday activities $(114,289)$, or that they are not well prepared for sun protection in these settings (296). Parental awareness about sun exposure risk in multiple settings is therefore highly important to minimize sunburn risk, even among parents reporting high levels of sun protection behaviours.

High levels of sun protection behaviours were observed, with the majority of parents frequently reporting shade-seeking and clothing behaviours, in line with sun safety recommendations. This finding contrasts with earlier studies using comparable measures $(48,76,156)$. The observed discrepancy between high reported sun protection behaviours and substantial sunburn risk is noteworthy. This may indicate that, while parents may believe they are adhering to sun safety recommendations, the actual execution is suboptimal. Several studies reported on the incompleteness of sun protection measures for children. For example, sunscreen efficacy depends mainly on correct application patterns (297). However, sufficient sunscreen application seems particularly difficult for parents (296), as indicated by their failure to apply sunscreen 30 minutes before going outdoors (76), by skipping sensitive body parts (195), applying insufficient amounts of sunscreen (298) or not reapplying every two hours (50). Parents' erroneous perception that they have sufficiently protected their child with sunscreen may lead them to prolong their time spent in the sun, resulting in sunburn (50, 193). Further, studies reported that children are less likely to be protected by sunglasses and a hat than with a T-shirt $(280,299)$, while concurrent use is recommended (6). Hence, it is possible that the parents in the current study overestimated their adherence to specific sun protection recommendations, despite the information provided regarding sufficient sun protection in the questionnaire. Another explanation for this finding could be that parents were unaware of their children's skin sensitivity or that they underestimated previous sunburn experiences $(119,206,296)$.

Although research on sun exposure patterns in children is scarce, recent studies from Germany reveal that the amount of time that very young children (aged 3 to 6 ) spend outside during peak UV hours in various settings was high (78), and that older children receive high doses of UVR in playgrounds (168). Our findings also suggest high levels of exposure, in particular among young children, in both sun exposure situations. The parents of young children reported adequate sun protection behaviours when their children were highly exposed in planned situations, but this was not echoed in low sunburn risk. A lower sunburn risk was however found among young children whose parents limited their sun exposure in planned situations. These findings emphasize the difficulty of optimal sun protection in planned sun exposure situations and may lead to the question of whether sunburn prevention is entirely possible or realistic in such situations. Protecting children sufficiently on the beach or by the swimming pool is particularly challenging, since 
availability of shaded areas is often lacking, sunscreen has to be reapplied more frequent clothing is perceived as burdening $(166,296)$.

Parents assigned to the best-performing class had the youngest children with more sensitive skin complexions. The higher protection for younger children is in agreement with previous research $(78,156$, 189). Since sun protection attitudes and behaviours decrease during adolescence and sunburn prevalence increases, establishing sun safety for older children is considered highly important $(48,79,98,257)$. Increased parental vigilance and sun protection behaviours among children with more sensitive skin types has also been described in earlier work $(119,284,289,296)$. Similarly, parents with less sensitive skin complexions may assume that their skin phenotype protects them from UVR (74), resulting in them providing less sufficient sun protection for their children (76). The association of children's sun-sensitivity with better sun protection and thus lower sunburn rates might seem obvious, but is often not apparent $(48,50,247)$. In the present study, a comparable relation between more sensitive skin types and high parental sun protection behaviours was found. Despite this, these children still seemed at high-risk for sunburn. The association between children's skin sensitivity, sun protection behaviours and their sunburn risk however deserves closer examination in order to compose future sun safety interventions.

\section{Strengths and limitations}

This study has strengths that are worth mentioning. Firstly, the assessment of comprehensive sun protection behaviours in distinctive sun-exposing situations provides a novel and insightful approach compared to existing parental sun protection research. Secondly, by using a large sample size and including more than five indicators, the validity of the latent class fitting was optimized.

The task of exploring associations between sunburn, sun exposure and sun protection behaviours is not straightforward and is charged with some methodological challenges. Firstly, although sunburn rates in our study sample were comparable or even higher than reported sunburn in the literature, performance of parental sun protection behaviours was high. A social desirability bias could have been at play, urging parents to report more frequent protection behaviours. Further, since all questions were asked regarding the previous year, recall bias may have limited the accuracy of the self-reported behaviours (300). Despite our efforts to reduce these biases by stressing confidentiality and non-disclosure of the study purpose, these biases should be considered when interpreting the present findings. Secondly, sun exposure was broadly defined in this study, and details such as weather conditions, exact time of day and duration of activities were not taken into account. Self-reported sun protection has been shown to be a valid measure when contextual factors as time and activities are specifically assessed (301), or when the retrospective timeframe is as short as possible (272). Future assessment of sun exposure should ideally include tailored questions regarding specified sun-exposing situations. Lastly, the findings in this study may not readily be generalised to Dutch parents since the majority of parents received a high level of education and most children were aged between 4 and 6 years.

\section{Implications}

The majority of parents seemed to perform recommended sun protection strategies frequently, but a variability in sun protection behaviours and sun exposure patterns were also observed. Further, specific individual characteristics such as skin type and children's age explained such variability in part. Despite the emergence of a positive picture of sun protection behaviours, a high sunburn risk was still prevalent among the majority of children. These exploratory findings hint at directions for further research in order to investigate children's sunburn in more detail.

First, all indicators were assessed subjectively. For future efforts, we recommend the application of more objective measurements of sunburn and received UVR-exposure as well as performance of sun protection behaviours. For example, some parents have difficulty recognising sunburn as such, e.g. being unaware that 
reddening of the skin already signifies sunburn, leading to an underestimation of sunburn prevalence (296). Objectively assessing sunburn and its severity would be worthwhile to improve risk-profiling (e.g. through biometric measures of melanin and erythema on the skin (302)), since sunburn and particularly severe and painful sunburn are highly correlated with melanoma development in later life (303). Further, although validation studies show fair to strong correlations between self-reported and observed exposure $(272,304)$, collecting objective data about children's UVR-exposure has been strongly advocated (93). Environmental effects such as shade coverage, altitude or humidity cannot be measured in self-reported questionnaires (89). Because detailed information on actual received UVR-dosage is lacking among children, the formulation of specific policy and guidelines, weighted for specific environmental characteristics (e.g. latitude, cloudiness, seasonality), cannot be formulated. As stated, parents may feel obliged to report desirable sun protection behaviours, or may overestimate their behaviours, which can both affect the validity of the findings. Investigating the execution of specific sun protection measures (e.g. using $\geq S P F 30$, reapplication patterns (297) and wearing sunglasses as well as a hat) could provide further insight in the execution of parental sun protection behaviours.

Second, since the findings imply that particularly planned exposure (e.g. beach or swimming pool) may increase the risk of sunburn, implementing health-promoting environmental cues in these settings are advisable $(166,305)$. For instance, by providing shade, promotion of UV-protective clothing and freely available sunscreen, both parents and children can be encouraged to perform sun protection behaviours more effectively.

Third, similar to earlier studies $(80,156,306)$, the present study revealed that older children seem to receive less protective behaviours from their parents, and experience more sunburn than younger children. Future interventions should target older children, especially before entering the adolescent phase $(120,247)$.

\section{Conclusions}

This study emphasizes the complexity of the association between children's sunburn, their sun exposure and parental sun protection behaviours while indicating directions for further investigation. The findings indicate that despite fair to good reported sun protection behaviours, sunburn occurrence was still high among children. Furthermore, highest sun protection was reported for younger children with more sensitive skin types, which was reflected in lower sunburn risk among a small group of children. We were unable to fully unravel the underlying mechanisms of the associations between sun exposure, sun protection behaviours and sunburn. Therefore, recommendations for increasing sun safety in children are provided with a cautionary note. Further research is required to clarify these complex relations in order to improve or develop tailored interventions. 
Table 4. All model parameter estimates of Multinomial Linear Regressions

\begin{tabular}{|c|c|c|c|c|c|c|}
\hline & Effect & Estimate & SE & Exp(B) & Wald & p value \\
\hline \multirow[t]{18}{*}{ Class 2} & Intercept & -1.02 & .46 & & 5.00 & .025 \\
\hline & Province (North) & -.40 & .39 & .67 & 1.08 & .30 \\
\hline & Province (East) & -.11 & .26 & .89 & .18 & .67 \\
\hline & Province (West) & -.05 & .24 & .95 & .04 & .84 \\
\hline & Education (Low) & -.18 & .27 & .84 & .44 & .51 \\
\hline & $\begin{array}{l}\text { Education } \\
\text { (Intermediate) }\end{array}$ & .41 & .21 & 1.51 & 4.02 & .052 \\
\hline & Parental age (Young) & .42 & .36 & 1.52 & 1.39 & .24 \\
\hline & $\begin{array}{l}\text { Parental age (Middle- } \\
\text { aged) }\end{array}$ & .05 & .24 & 1.05 & 0.46 & .83 \\
\hline & Parental sex (Male) & -.28 & .19 & .76 & 2.13 & .14 \\
\hline & Parental skin type (I) & 1.05 & .55 & 2.87 & 3.71 & .054 \\
\hline & Parental skin type (II) & .26 & .40 & 1.30 & .43 & .51 \\
\hline & Parental skin type (III) & .24 & .39 & 1.27 & .37 & .54 \\
\hline & Child's age (Young) & 1.18 & .26 & 3.24 & 20.47 & .00 \\
\hline & $\begin{array}{l}\text { Child's age (Middle- } \\
\text { aged) }\end{array}$ & .44 & .26 & 1.55 & 2.76 & .10 \\
\hline & Child's sex (Boy) & -.11 & .18 & .90 & .35 & .55 \\
\hline & Children's skin type (I) & 1.35 & .63 & 3.86 & 4.67 & .03 \\
\hline & Children's skin type (II) & .46 & .39 & 1.58 & 1.41 & .24 \\
\hline & Children's skin type (III) & .19 & .37 & 1.21 & .26 & .61 \\
\hline \multirow[t]{16}{*}{ Class 3} & Intercept & .27 & .37 & & .57 & .45 \\
\hline & Province (North) & -.14 & .31 & .87 & .19 & .66 \\
\hline & Province (East) & -.42 & .22 & .66 & 3.46 & .06 \\
\hline & Province (West) & -.24 & .20 & .79 & 1.47 & .23 \\
\hline & Education (Low) & -.11 & .22 & .89 & .26 & .61 \\
\hline & $\begin{array}{l}\text { Education } \\
\text { (Intermediate) }\end{array}$ & .38 & .18 & 1.46 & 4.62 & .32 \\
\hline & Parental age (Young) & .28 & .31 & 1.32 & .78 & .38 \\
\hline & $\begin{array}{l}\text { Parental age (Middle- } \\
\text { aged) }\end{array}$ & -.00 & .19 & 1.00 & .00 & .99 \\
\hline & Parental sex (Male) & -.24 & .16 & .79 & 2.26 & .13 \\
\hline & Parental skin type (I) & 1.17 & .47 & 3.23 & 6.18 & .01 \\
\hline & Parental skin type (II) & .28 & .32 & 1.32 & .74 & .39 \\
\hline & Parental skin type (III) & .44 & .31 & 1.55 & 1.96 & .16 \\
\hline & Child's age (Young) & .48 & .21 & 1.6 & 5.20 & .02 \\
\hline & $\begin{array}{l}\text { Child's age (Middle- } \\
\text { aged) }\end{array}$ & .24 & .20 & 1.27 & 1.34 & .25 \\
\hline & Child's sex (Boy) & -.10 & .15 & .91 & .41 & .52 \\
\hline & Children's skin type (I) & 1.32 & .56 & 3.74 & 5.47 & .02 \\
\hline
\end{tabular}




\begin{tabular}{|c|c|c|c|c|c|c|}
\hline & Children's skin type (II) & .56 & .32 & 1.75 & 3.10 & .08 \\
\hline & Children's skin type (III) & .36 & .30 & 1.44 & 1.47 & .23 \\
\hline \multirow{18}{*}{ Class 4} & Intercept & -1.51 & .68 & & 5.00 & .03 \\
\hline & Province (North) & -.60 & .83 & .55 & .53 & .47 \\
\hline & Province (East) & -.36 & .52 & .70 & .48 & .49 \\
\hline & Province (West) & .59 & .41 & 1.80 & 2.06 & .15 \\
\hline & Education (Low) & .47 & .43 & 1.60 & 1.20 & .27 \\
\hline & $\begin{array}{l}\text { Education } \\
\text { (Intermediate) }\end{array}$ & .51 & .36 & 1.67 & 2.10 & .15 \\
\hline & Parental age (Young) & -.36 & .62 & .70 & .33 & .56 \\
\hline & $\begin{array}{l}\text { Parental age (Middle- } \\
\text { aged) }\end{array}$ & -.61 & .36 & .55 & 2.81 & .09 \\
\hline & Parental sex (Male) & .07 & .32 & 1.08 & .05 & .82 \\
\hline & Parental skin type (I) & -.40 & .95 & .67 & .18 & .67 \\
\hline & Parental skin type (II) & -.96 & .60 & .38 & 2.57 & .11 \\
\hline & Parental skin type (III) & -.04 & .53 & .96 & .01 & .94 \\
\hline & Child's age (Young) & .21 & .45 & 1.24 & .23 & .63 \\
\hline & $\begin{array}{l}\text { Child's age (Middle- } \\
\text { aged) }\end{array}$ & .69 & .40 & 2.00 & 3.01 & .08 \\
\hline & Child's sex (Boy) & -.18 & .31 & .84 & .33 & .57 \\
\hline & Children's skin type (I) & .02 & 1.23 & 1.02 & .00 & .99 \\
\hline & Children's skin type (II) & .05 & .59 & 1.05 & .01 & .94 \\
\hline & Children's skin type (III) & -.03 & .53 & .97 & .00 & .95 \\
\hline
\end{tabular}


chapter six

\section{Are environmental interventions targeting skin cancer prevention among children and adolescents effective? A systematic review}



Skin cancer, which is increasing exceedingly worldwide, is substantially preventable by reducing unprotected exposure to ultraviolet radiation (UVR). Several comprehensive interventions targeting sun protection behaviours among children and adolescents in various outdoor settings have been developed; however, there is a lack of insight on stand-alone effectiveness of environmental elements. To compose future skin cancer prevention interventions optimally, identification of effective environmental components is necessary. Hence, an extensive systematic literature search was conducted, using four scientific databases and one academic search engine. Seven relevant studies were evaluated based on stand-alone effects of various types of environmental sun safety interventions on socio-cognitive determinants, sun protection behaviours, UVR exposure, and incidence of sunburns and nevi. Free provision of sunscreen was most often the environmental component of interest, however showing inconsistent results in terms of effectiveness. Evidence regarding shade provision on shade-seeking behaviour was most apparent. Even though more research is necessary to consolidate the findings, this review accentuates the promising role of environmental components in skin cancer prevention interventions and provides directions for future multi-component sun safety interventions targeted at children and adolescents in various outdoor settings.

\section{Introduction}

Melanoma and non-melanoma skin cancers (NMSC) are two of the most rapidly increasing cancer types among white populations (14). Since the early 1980s, melanoma incidence rates have risen twofold in the United States to even threefold in Europe $(15,28,149,208)$. On a global level, more than 55,000 people died from melanoma in 2012, with the greatest burden in Europe, the United States, Australia, and New Zealand $(209,307)$. Continuation of the rising incidence rates of melanoma is predicted until 2022 at least in the United States and among European countries (208), also implying increased health care costs and need for a new skin cancer disease management strategy $(38,150)$. Even though exposure to ultraviolet radiation (UVR) is important for production of vitamin D (308), sun exposure and sunburns during early childhood are the most important risk factors for developing melanoma in later life $(42,45,59,309,310)$ and should therefore be limited $(96,255)$. Objective data about the overall time children are exposed to UVR are inconsistent and vary per age group, latitude, and country of origin (169, 311-313). Even though insight in specific settings where children spend their outside time nowadays is scarce, children seem to often engage in outdoor activities at (pre)school $(43,46)$, around the house and around the beach or swimming pools $(80,314)$, when playing in outdoor playgrounds $(315,316)$ or in public parks $(317)$. Compared to adolescents, younger children spend time outside after school and during the weekend to a greater extent $(43,255)$.

The Surgeon General and World Health Organization (WHO) have documented five guidelines to enhance sun protection in a call to action addressing the rapidly increasing skin cancer incidence rates. These behaviours consist of wearing protective clothing, wearing sunglasses and a hat, seeking shade, avoiding peak sunlight hours, and applying sunscreen (6). Over the years, several types of interventions have been developed to encourage various sun protection behaviours among parents and children. Educational interventions for example, can be individually directed and primarily focus on changing intentional decision-making processes (109), by increasing one's knowledge, improving socio-cognitive determinants (e.g., attitudes, self-efficacy) and learning skills to perform a certain behaviour. Positive effects of such interventions have been shown for different sun protection behaviours $(82,159)$ and on knowledge, attitudes, intentions, and behaviour in various settings $(158,159)$ for children and adolescents $(318,319)$. 
Although educational interventions have demonstrated positive effects, it is important to acknowledge that behaviour can also be automatically triggered by environmental characteristics (164). This is illustrated by dual process models, which state that behaviour can both consciously and automatically be influenced by one's physical environment, such as by physical adaptations, policy or both (112). One's environment can be characterized by both different levels of influence (e.g., micro/family setting or macro/community level) as by different types of the environment (e.g., political, economic, social, or physical). Hence, adapting the environment where children and adolescents are highly exposed to UVR can affect sun protection behaviour (112).

Overall evidence for effectiveness of multi-component sun safety interventions, integrating both behavioural and environmental strategies, among children and adolescents in outdoor settings, is restricted (143). Moreover, insight in effects of autonomous elements of these interventions is lacking and additional research is necessary. Since childhood is an important phase where consolidation of health behaviour takes place (320) and life-long sun protection habits can be established (71), effectiveness of these interventions needs further examination.

In conclusion, a comprehensive approach in skin cancer prevention strategies among children, targeting both behavioural and environmental factors, is needed. Yet, in order to compose a mix of effective strategies targeting both types of factors and therefore design future interventions optimally, identifying the effects of separate components targeting these factors is necessary. Hence, the aim of this review was to systematically investigate available literature concerning stand-alone effectiveness of environmental interventions targeting sun protection behaviours among children and adolescents in various outdoor settings.

\section{Methods}

\section{Search strategy}

The PRISMA guidelines for systematic reviews were followed to enhance reproducibility of this study (321). The formulated research question included study characteristics according to the PICOS tool, affirmed by the Cochrane Collaboration (322). Development of the search strategies was accomplished with help of a scientific information specialist (author SJ). Since the setting where an intervention took place was specifically important, 'setting' characteristics replaced the standardized 'comparison' element out of the standardized PICOS in the search strings. An extensive literature search regarding environmental sun safety interventions was conducted using four databases (i.e., PubMed, PsycInfo, Cochrane, Web of Science) and one academic search engine (i.e., Google Scholar) applying systematic formulated search strings. The search strings contained five index terms: (1) population, (2) intervention, (3) setting, (4) outcomes, and (5) study design. These databases together covered a broad range of health, behavioural, and social science subjects as well as the ability to search for scholarly literature. Examples of two search strings are depicted in Appendix A.

\section{Eligibility criteria}

Prior formulated inclusion criteria were for studies looking at: (1) stand-alone effects on socio-cognitive determinants, sun safety behaviours, UVR exposure, and sunburn and nevi incidence; (2) physical environmental, policy, economic, and/or socio-cultural interventions (37); (3) outdoor and school settings; (4) among children aged between 0 and 18 years; (5) including intervention designs with at least one comparison group, were eligible for inclusion. Stand-alone effectiveness of environmental interventions was interpreted as such that the sun safety intervention, whether it represented a single or multi-component intervention, should consist of one separate intervention arm in which the environmental component was 
exclusively tested. Inclusion and exclusion criteria are depicted in Table 1. Moreover, studies that were conducted before 1990 and were written in non-English were omitted. No filters were used in the databases and the academic search engine since the search strings were optimally and carefully designed and inclusion and exclusion criteria were clear.

Table 1. Index terms (PICOS), inclusion- and exclusion criteria

\begin{tabular}{|c|c|c|c|c|c|}
\hline & Population & Intervention & Comparison & Outcomes & Study design \\
\hline $\begin{array}{l}\text { Inclusion } \\
\text { criteria }\end{array}$ & $\begin{array}{l}\text { Infants, toddlers, } \\
\text { preschool } \\
\text { children, } \\
\text { children, and } \\
\text { adolescents }\end{array}$ & $\begin{array}{l}\text { Environmental } \\
\text { adaptations } \\
\text { targeted on } \\
\text { sun safety } \\
\text { behaviours } \\
\text { and skin } \\
\text { cancer } \\
\text { prevention }\end{array}$ & $\begin{array}{l}\text { Interventions } \\
\text { that enable } \\
\text { assessment of } \\
\text { stand-alone } \\
\text { effects of } \\
\text { environmental } \\
\text { adaptations, } \\
\text { using a control } \\
\text { group }\end{array}$ & $\begin{array}{l}\text { Effectiveness of } \\
\text { environmental } \\
\text { adaptations on } \\
\text { socio-cognitive } \\
\text { determinants, } \\
\text { sun safety } \\
\text { behaviours, } \\
\text { UVR exposure, } \\
\text { sunburn } \\
\text { incidence and } \\
\text { nevi }\end{array}$ & $\begin{array}{l}\text { Randomized } \\
\text { Controlled } \\
\text { Trials and } \\
\text { (quasi-) } \\
\text { experimental } \\
\text { designs to } \\
\text { objectify } \\
\text { effects of } \\
\text { interventions }\end{array}$ \\
\hline $\begin{array}{l}\text { Exclusion } \\
\text { criteria }\end{array}$ & $\begin{array}{l}\text { A target } \\
\text { population of } \\
\text { adults or elderly } \\
\text { with an age of } \\
18 \text { or above, a } \\
\text { population in } \\
\text { which children } \\
\text { could not be } \\
\text { differentiated, } \\
\text { children with } \\
\text { skin diseases, } \\
\text { hospitalized } \\
\text { children and } \\
\text { childhood } \\
\text { cancer survivors }\end{array}$ & $\begin{array}{l}\text { Interventions } \\
\text { without } \\
\text { environmental } \\
\text { components } \\
\text { and/ or } \\
\text { educational } \\
\text { interventions } \\
\text { only }\end{array}$ & $\begin{array}{l}\text { Interventions } \\
\text { without a control } \\
\text { group and/ } \\
\text { or combined } \\
\text { interventions } \\
\text { without } \\
\text { exclusively } \\
\text { investigating } \\
\text { effects of } \\
\text { environmental } \\
\text { adaptations }\end{array}$ & $\begin{array}{l}\text { Outcome } \\
\text { variables not } \\
\text { related to } \\
\text { socio-cognitive } \\
\text { determinants, } \\
\text { sun safety } \\
\text { behaviours, } \\
\text { UVR exposure, } \\
\text { sunburn } \\
\text { incidence and } \\
\text { nevi }\end{array}$ & $\begin{array}{l}\text { Study } \\
\text { designs } \\
\text { without a } \\
\text { comparison } \\
\text { group } \\
\text { and study } \\
\text { protocols }\end{array}$ \\
\hline
\end{tabular}

\section{Study selection}

The systematic search took place from the 17 August to the 4 October 2018. An updated search to ensure inclusion of all available recent studies was performed on the 17 September 2019. After eliminating duplicate studies according to the method described by Bramer and colleagues (323), title selection of the studies took place by the first (KT) and last (FS) author in the first round. Consensus between KT and FS about selected studies based on titles needed to be reached before continuing to the next stage. In the second round, eligible abstracts were selected. In the third round, full-text articles were selected. When discrepancies between the two researchers were observed based on the title or abstract, the paper was taken to the second or third round in the study selection process. Both KT and FS determined upon final selection of relevant articles that were used for data abstraction and disagreements were discussed. Furthermore, when no consensus about eligibility could be reached, a third researcher (author LO) was consulted. 


\section{Data abstraction}

Study characteristics and study outcomes were extracted from the selected studies and quality assessments to estimate the risk of bias were performed. Descriptive information about detailed study characteristics, study outcomes and data regarding quality of studies were reviewed independently before abstracting relevant data.

\section{Study characteristics}

A standardized data abstraction form (324) was critically examined and altered regarding specific characteristics of the studies that were selected, in consensus with the study objectives. Characteristics of the selected studies that were abstracted were predominantly formulated based on the PICOS framework and can be found in Table 2. After entirely reading the first included study (325), study characteristics were further specified according to elaborate data that was present in this study.

\section{Study outcomes}

Study outcomes were regarded important for extraction based on previous systematic reviews $(82,143)$ and were related to socio-cognitive determinants, sun safety behaviours, UVR exposure, and health-related outcomes (i.e., reported sunburns and/or melanocytic nevi (i.e., moles)). The behavioural outcomes were based on recommended sun safety guidelines $(6,68)$. Since occurrence of sunburn and melanocytic nevi are both objectifications of (over)exposure to UVR, these were included as outcome measures (82). Finally, information about statistical analyses that were conducted, statistical results, reported stand-alone effects of the intervention and if applicable, reported effect sizes were also abstracted in order to gain an overview of relevant study data.

\section{Study quality and risk of bias}

The quality of included studies was assessed by using the Quality Assessment Tool for Quantitative Studies from the Effective Public Health Practice Project (EPHPP) (326), which is a validated tool to use in the assessment of quality of studies $(327,328)$. With regard to assessing the quality of studies included in the review, methodological characteristics were rated as 'strong', 'moderate', or 'weak'.

\section{Results}

In total, 1085 articles were found in the five databases and were screened for eligibility. After the deduplication process, 753 titles remained. After screening of titles and abstracts, 45 articles were eligible for full-text screening. In the full-text phase, three authors were approached to retrieve more information upon deciding whether these articles were eligible for data abstraction, since follow-up data was not present (329-331). The first authors of these studies were contacted to investigate whether data were available. In one case, a study protocol described the methods for conducting a study in which effects of shaded areas in public parks was investigated from 2013 to 2016 (330). The first author of this article mentioned that the data would be submitted on a short notice and that follow-up results were presented at two conferences. The follow-up data of this study was therefore included in the analysis as a conference abstract. Another study (331) only described baseline data. After contacting the first author, it was mentioned that follow-up data was retrieved and presented at a conference in 2010, however publication was not expected to be established anytime soon. Therefore, the data from this study were included as a conference abstract too (332). Finally, the follow-up data of one study (329) was eventually excluded in the analysis after contacting the authors, since stand-alone effects could not be distinguished for children or adolescents specifically. 
Ultimately, from the 45 articles, eight studies that described the stand-alone effects of environmental interventions were qualified for data-abstraction (see Appendix B for the flowchart). After excluding the last article (329), this resulted in a total of seven studies that were used in the data-extraction process. A full overview of study outcomes and results is provided in Table 3.

\section{Study results}

\section{Types of environmental components}

In most of the studies, an economic intervention (333) was included in an intervention-arm. More specifically, proactive provision of free sunscreen was the most applied environmental component. In three studies free sunscreen was the exclusive addition in the environmental intervention-arm, while in one study sunscreen provision was one of multiple components. In two studies, bottles of sunscreen were provided to parents at school near the end of the school year (334) or day-care centre before spring (335). In one study, sunscreen was handed out to children themselves, at the end of the school year (336). Lastly, free sunscreen was provided among other factors (portable shade tents, posters, and policy consultation), in which sunscreen dispensers were placed in city parks, community centres and outdoor recreation sites (325). In another economic intervention, provision of protective clothing was used (332). The entire intervention was based on arrangement of clothing, hats, as well as swim shirts for children attending day care centres.

Subsequently, the second most used intervention type was an adaptation in the physical environment, consisting of shade provision in three studies (333). The effectiveness of shade sails at secondary school sites (337) and purpose-built shade and trees covering public park areas (338) were investigated. Moreover, effects of portable tents, besides sunscreen dispensers, were examined in one study (325).

\section{Effects of environmental interventions on socio-cognitive determinants}

One study described socio-cognitive determinants among other outcomes (336). In this case, the environmental intervention consisted of free provision of sunscreen. Children showed a significantly greater reduction in their desire to have a tan in comparison with children from the other intervention and control group. No effects were found on knowledge and awareness.

\section{Effects of environmental interventions on sun safety behaviours and UVR exposure}

Six studies had one or more sun safety behaviours as outcome(s); UVR exposure was part of the outcome measures in two studies $(334,335)$. Two studies solely assessed shade-seeking behaviour $(337,338)$ and one study focused on sunscreen use (334). In the remaining studies more than one sun safety behaviour was measured $(325,335,336)$. Parents estimated their children's execution of sun safety behaviours in most of the studies $(325,334-336)$. In one study, parents and children were both assessed (336). The effects of different types of environmental interventions on sun safety behaviours were variable. For instance, economic interventions consisting of free sunscreen or sunscreen dispensers did not account for significant improvements in parental or children's sun safety behaviours or UVR exposure (334-336). Physical changes in the environment, consisting of shaded areas and purposely planted trees, showed positive effects on shade-seeking behaviour of children and adolescents in two studies $(337,338)$. However, in a study where sunscreen dispensers were combined with shaded areas, behavioural effects were absent (325).

\section{Effects of environmental interventions on melanocytic nevi and sunburns}

One study assessed nevi incidence as exclusive outcome (331), two studies evaluated incidence of nevi among other outcomes (334, 335), and one study investigated sunburn incidence (336). Findings regarding the effects of economic interventions on nevi and sunburn incidence, such as free provision of sunscreen, were contradicting. One study found that children who received free sunscreen had developed less nevi at the end of the intervention period (334), whereas another study found no effects of free sunscreen provision on nevi incidence (335). Lastly, in the only study that investigated the effects of free sunscreen provision on sunburn incidence, no evidence for effects was found (336). In the only study that investigated free provision of UV-protective clothing, hats, and swim shirts (332), findings show that children who did not receive the intervention developed higher incidence of moles on their bodies than children in the intervention group. 


\section{Quality assessment of studies}

Weak coding was most often due to absence of reported controlling for confounding variables in study designs or statistical analyses. Furthermore, data collection methods were coded as weak in two studies, due to the lack of validated questionnaires $(325,336)$. Drop-out rates were above $40 \%$ in two studies, resulting in a weak coding for this sub-item $(325,336)$. All studies were randomized or cluster controlled trials, except for one non-randomized trial with pre- and post-tests (338). Two studies guaranteed blinding in which the outcome assessors were not aware of the intervention status of participants $(334,335)$ and in two studies, blinding of assessors was not possible due to observational methods $(337,338)$. In most studies, respondents were not aware of the research question. In one study, the blinding procedure was not explained (332). Notably, none of the included studies reported effect sizes between intervention and control groups. Since the included studies were heterogeneous in terms of intervention type, outcomes, and statistical results and specific statistical information was absent in three studies, a pooled effect size was not calculated. See Table 4 for the quality rating per study.

Table 4. Study quality of included studies $(326,328)$

\begin{tabular}{|l|l|l|l|l|l|l|}
\hline & $\begin{array}{l}\text { Selection } \\
\text { bias }\end{array}$ & Study design & Confounders & Blinding & $\begin{array}{l}\text { Data } \\
\text { collection } \\
\text { method }\end{array}$ & $\begin{array}{l}\text { Withdrawals } \\
\text { and drop-outs }\end{array}$ \\
\hline $\begin{array}{l}\text { Gallagher et } \\
\text { al., 2000 }\end{array}$ & Moderate & Strong & Weak & Moderate & Strong & Moderate \\
\hline $\begin{array}{l}\text { Glanz et al., } \\
2000\end{array}$ & Moderate & Strong & Strong & Moderate & Weak & Weak \\
\hline $\begin{array}{l}\text { Barankin et } \\
\text { al., 2001 }\end{array}$ & Strong & Strong & Weak & Moderate & Weak & Weak \\
\hline $\begin{array}{l}\text { Bauer et al., } \\
2005\end{array}$ & Strong & Strong & Strong & Strong & Strong & Moderate \\
\hline $\begin{array}{l}\text { Dobbinson et } \\
\text { al., 2009 }\end{array}$ & Weak & Strong & Weak & Moderate & Strong & Strong \\
\hline $\begin{array}{l}\text { Harrison et } \\
\text { al., 2010 }\end{array}$ & Moderate & Strong & Weak & Weak & Strong & Strong \\
\hline $\begin{array}{l}\text { Dobbinson et } \\
\text { al., 2019 }\end{array}$ & Moderate & Moderate & Strong & Moderate & Strong & N/A \\
\hline
\end{tabular}

\section{Discussion}

This systematic literature study is the first to examine stand-alone effects of environmental sun safety interventions among children and adolescents on socio-cognitive determinants, sun safety behaviours, UVR exposure, development of nevi, and sunburn incidence. Seven studies were included, showing that free provision of sunscreen (four times), shade supply (three times) and provision of UV-protective clothing and accessories (one time) were the environmental types of interventions implemented. Five studies showed significant effects of environmental components, assessed after one year on average (yet ranging from four months to three years). Positive effects were especially visible on shade-seeking behaviour and incidence of nevi; effects on socio-cognitive determinants, other sun safety behaviours, UVR exposure, and sunburn incidence were not evident. Overall, shade provision seemed to show the most encouraging results. Recent 
reviews about the effects of shade provision on sun safety behaviours among adults show encouraging results as well $(144,167,339)$.

First, in respect of environmental interventions implemented in the studies included, five used an economic component such as free provision of sunscreen (334-336), free sunscreen dispensers (325), or free provision of UV-protective clothing and garments (332). Physical changes in the environment such as supplying shaded areas, portable shade tents, or planted trees were used in three studies $(325,337,338)$. Furthermore, sunscreen provision was the most frequently mentioned intervention, even though sunscreen use alone is not sufficient for UVR protection $(194,216)$ and is currently considered an additional recommendation besides other sun safety practices $(6,340)$. Despite the fact that provision of sunscreen is relatively accessible and low-cost, economic interventions such as provision of clothing, hats, and sunglasses warrant further exploration as well $(90,341)$, since these methods seem to be more effective than sunscreen application $(194,214)$.

Second, when looking at the outcomes of the included studies, results showed that most outcomes were based on internationally recommended sun safety behaviours $(6,68)$, in which sunscreen use was regularly the primary behavioural outcome $(325,334-336)$, followed by shade-seeking $(337,338)$ and wearing UVprotective clothing $(325,335)$. The preference of measuring sunscreen use prior to shade-seeking and clothing behaviour in the studies is in accordance with a general popularity of sunscreen application (214). Even though it is encouraging that environmental sun safety interventions assess a variety of behavioural outcomes, results of the included studies show that actual effects of environmental interventions on behavioural change are scarce and were only found for shade-seeking behaviour (337, 338). These results accentuate the importance of enhancing other sun protection behaviours besides sunscreen use (6). Furthermore, effects of environmental interventions on other outcomes such as incidence of nevi were variable. Two studies showed positive results considering incidence of nevi on the body $(332,334)$. However, in these studies, no differences in UVR exposure between intervention and control groups were reported while UVR exposure is a great predictor for nevi development (46). Consistent conclusions about effects of environmental interventions on sunscreen use and incidence of nevi can therefore not be drawn.

Third, with regard to the setting in which interventions were implemented, the school setting (meso level) was most often used. This preference for the school setting is in conformity with the increased attention for school health promotion in general (342). Although the recognized Healthy Schools Approach is emerging internationally, attention for sun protection behaviour is often lacking (343-345). The promising results of comprehensive health promoting school programs emphasize the importance of sun safety in schoolsettings, with an added value of integrated environmental components (346). However, other settings can also be of considerable importance with regard to enhancing sun safety. As ecological systems theory implies, the macro level is also of great importance when targeting children's health behaviour (347). The minority of interventions directed at recreational venues (macro level) is therefore noticeable. Particularly, since the amount of time people spent at recreational settings is increasing among Western populations $(348,349)$. The amount of UVR exposure at these venues is often high (313) while sun protection is regularly lacking during outdoor activities (350-352) and no prevention policies are at place. Moreover, children specifically are at high risk of receiving large amounts of UVR at playgrounds due to unavailability of shaded areas, as revealed by a recent study conducted in Germany (168). These findings accentuate the importance of intervening in recreational settings. Nevertheless, more knowledge about specific locations where children receive the largest amounts of UVR is necessary before important settings for interventions can be defined, since insight in harmful sun exposure patterns is still lacking (353) and adequate measurement is challenging (354).

Although this review showed that economic types of interventions are most often implemented, it would also be advantageous to analyse the effect of other types of interventions as well. For example, policy intervention types were absent, while these strategies seem to gain positive effects on other health behaviours such as food intake and physical activity $(355,356)$. Examples of these types of interventions could be scheduling outdoor activities outside of UV peak hours (82), regulation of wearing hats and playing indoors or in the shade (140) or increasing the availability of shaded areas (357). 


\section{Strengths and limitations of the included studies}

A few strengths of the included studies are worth mentioning. Almost all studies used methodologically strong designs, mostly (cluster) RCT designs, which strengthens the validation of evidence (358). Furthermore, all studies used a relatively large sample size and drop-out rates were considered low. Most studies used multiple measurements which generated long-term data. Lastly, in the included studies, children of all age groups were represented.

Also, a few limitations should be mentioned. Quality components such as selection bias, handling confounders, and handling withdrawals and drop-out were often rated as weak to moderate (326). Furthermore, most weak ratings were given for controlling for confounders, which was often caused by an abstinence of information reported in the studies. Moreover, in some studies lack of details disabled the possibility to rate the study quality sufficiently, which may have resulted in inaccurate weak ratings. For example, data on statistical analyses performed were missing and contact with the author did not provide sufficient information (336). These methodological shortcomings together may have affected the validity of the results. Furthermore, no effect sizes were reported which made comparison of statistical impact between studies difficult. Due to heterogeneity, it was not applicable nor eligible to calculate comparative effect sizes between studies (359). Follow-up period was mixed in the studies reporting significant effects (ranging from three months to three years), thus making it difficult to assess the impact of various interventions. Lastly, even though it is regarded beneficial to include grey literature in a systematic review (360, 361), the results extracted from two conference abstracts $(332,338)$ should be interpreted with caution.

\section{Strengths and limitations of this review}

First, this review was conducted in accordance with common guidelines and with use of the PRISMA statement for performing and reporting systematic reviews (321,322), which includes relevant topics and concepts and enhances reporting of systematic reviews (362). Furthermore, five databases were used in the literature search which is a considerable amount of sources (359), which has accounted for avoiding missing relevant titles (363). Moreover, for assessing risk of bias of the included studies, the validated EPHPP tool was used $(326,328)$, which has proven adequate inter-rater agreement (364).

The current systematic review has also a few limitations. First, the amount of eligible studies that specifically met our criteria turned out limited, which complicates comparison between studies. However, since sampling was done systematically in this review with high sensitivity by formulating specific search strings for five databases, the sample size of available studies seems adequate to draw conclusions (363). Moreover, there is no golden standard stating a minimum of eligible studies to be included in the synthesis of systematic reviews. Lastly, years of publication of the included studies showed a shortage in recent studies conducted, which demonstrates a possible decrease in interest in environmental components in skin cancer prevention interventions.

\section{Recommendations}

Considering the results of this systematic review, several recommendations can be made. The large body of evidence for presence of health promoting sun safety interventions in general is positive, since health promoting adaptations in the environment show promising results on various other health behaviours (365-368), and the call for sun protection encouragement specifically is reported (6). Moreover, within skin cancer prevention specifically, a recent study revealed that three decennia of dissemination of the multi-component SunSmart program led to significant improvements in various sun protection behaviours (138). However, to gain knowledge on effectiveness of stand-alone effects of environmental interventions, it is recommended to investigate the effects of isolated components specifically in future studies. Albeit this review showed availability of a large amount of interventions that were initially eligible for inclusion, the absence of exclusively reported results of the environmental component(s) in most cases, restricted 
inclusion of those studies and therefore the ability to report extensively on the effectiveness of these components.

Second, sun protection behaviours of children themselves, in various settings, should be considered. Since children and adolescents were the target groups for this review, it is convenient that the responsibility of sun safety for children was expected to be among parents and teachers primarily. However, in a recent study (156), we found that children between approximately 11 and 14 years old, increasingly execute sun safety measures themselves, while parental protection towards their children declines. Hence, examination of children's own sun protection behaviours should certainly be included in future effect studies.

Third, the included studies in this review showed most studies were conducted in countries outside of Europe-inhabiting Caucasian populations, such as Oceania and Northern America, where high doses of ambient UVR exposure are present $(94,209)$. Since the incidence rates and melanoma risk have started risen earlier in these countries located at a lower latitude (19), it is understandable that skin cancer prevention strategies are already developed to a greater extent. Moreover, societal norms regarding UVR exposure might differ from those in European countries. Since latitudinal differences or seasonal variation can account for differences in need for sun protection strategies (369), more extensive research in which latitudinal differences are taken into account and, ideally performed in countries where skin cancer prevention is not yet normalized, is needed to translate research into practice. Specifically, since the need for skin cancer prevention due to rapidly growing incidence of melanoma in European countries is crucial $(150,370)$.

Fourth, in all studies, subjective measures such as self-report questionnaires and/or observations were used. To further increase validity, application of objective measures of UVR exposure, such as handheld meters and time-stamped dosimetry (371) and wrist worn dosimetry devices (169), is recommended. Especially, the combination of self-reported and personal dosimeter measurements is promising to consider in future studies (304).

In conclusion, this review demonstrated overall positive results of environmental interventions in five of the seven included studies. Among those, shade provision was the most promising and consistent in increasing shade-seeking behaviour. However, more research is necessary to investigate the perpetuation of these findings. As supplying shade provides intervention opportunities in various settings, in both schools and public areas, integrating shade provision in sun safety interventions for children is highly recommended. Moreover, future environmental interventions should focus more specifically on micro and macro levels of influence in children's social environments, such as the home- and recreational setting. 
Table 2. Study characteristics

\begin{tabular}{|c|c|c|c|c|c|c|c|}
\hline $\begin{array}{l}\text { Authors, } \\
\text { year }\end{array}$ & Country & $\begin{array}{l}\text { Target group, } \\
\text { recruitment }\end{array}$ & $\begin{array}{l}\text { Sample size } \\
\text { and setting }\end{array}$ & $\begin{array}{l}\text { Design (inter- } \\
\text { vention groups, } \\
\text { duration, ran- } \\
\text { domization) }\end{array}$ & $\begin{array}{l}\text { Intervention } \\
\text { type and level }\end{array}$ & Outcomes & $\begin{array}{l}\text { Outcome } \\
\text { measure- } \\
\text { ments }\end{array}$ \\
\hline $\begin{array}{l}\text { Gallagher et } \\
\text { al., } 2000\end{array}$ & Canada & $\begin{array}{l}\text { Target group } \\
\text { Elementary } \\
\text { school children } \\
\text { aged between } \\
6-7 \text { and 9-10 } \\
\text { years } \\
\text { Respondents } \\
\text { Children and } \\
\text { their parents } \\
\text { Recruitment } \\
\text { School princi- } \\
\text { pals were first } \\
\text { approached for } \\
\text { study partici- } \\
\text { pation. Parents } \\
\text { were then } \\
\text { asked for in- } \\
\text { formed consent } \\
\text { for enrolling } \\
\text { their child in the } \\
\text { study }\end{array}$ & $\begin{array}{l}6 \text { elementary } \\
\text { schools in } \\
\text { Vancouver } \\
458 \text { children } \\
\text { at baseline } \\
309 \text { children } \\
\text { at follow-up } \\
(67,5 \%)\end{array}$ & $\begin{array}{l}\text { Design } \\
\text { Two-arm rand- } \\
\text { omized trial } \\
\text { Intervention } \\
\text { groups } \\
\text { 1. Control group } \\
\text { (no intervention; } \\
\text { 164 children) } \\
\text { 2. Sunscreen } \\
\text { intervention (145 } \\
\text { children) } \\
\text { Duration } \\
\text { Three years } \\
\text { Baseline (June } \\
\text { 1993) } \\
\text { Three post-tests } \\
\text { (end of summer } \\
\text { season in 1994, } \\
\text { 1995 and May } \\
\text { 1996) } \\
\text { Randomization } \\
\text { Children were } \\
\text { randomized in } \\
\text { either control } \\
\text { or intervention } \\
\text { group by a stat- } \\
\text { istician }\end{array}$ & $\begin{array}{l}\text { Intervention type } \\
\text { Economic } \\
\text { Intervention level } \\
\text { Meso } \\
\text { The environmen- } \\
\text { tal component } \\
\text { consisted of } \\
\text { provision of a } \\
\text { broad spectrum } \\
\text { sunscreen bottle } \\
\text { (SPF 30), provid- } \\
\text { ed at the parents } \\
\text { at the end of } \\
\text { each school } \\
\text { year. Instructions } \\
\text { and information } \\
\text { about frequency } \\
\text { of application } \\
\text { and sunscreen } \\
\text { amount were } \\
\text { included } \\
\text { The control } \\
\text { group did not } \\
\text { receive an } \\
\text { intervention } \\
\text { component }\end{array}$ & $\begin{array}{l}\text { Application } \\
\text { of sunscreen, } \\
\text { number of } \\
\text { counted nevi } \\
\text { on the body } \\
\text { and sun } \\
\text { exposure }\end{array}$ & $\begin{array}{l}\text { Nevi incidence } \\
\text { was measured } \\
\text { by physical } \\
\text { examination } \\
\text { from phy- } \\
\text { sicians and } \\
\text { sun exposure } \\
\text { was meas- } \\
\text { ured with } \\
\text { activity-based } \\
\text { question- } \\
\text { naires, } \\
\text { combined } \\
\text { with minimal } \\
\text { erythemal } \\
\text { dose (MED) } \\
\text { information } \\
\text { about sky } \\
\text { conditions, } \\
\text { latitude and } \\
\text { month of the } \\
\text { year }\end{array}$ \\
\hline $\begin{array}{l}\text { Glanz et al., } \\
2000\end{array}$ & $\begin{array}{l}\text { United } \\
\text { States }\end{array}$ & $\begin{array}{l}\text { Target group } \\
\text { Children aged } \\
\text { between 6-8 } \\
\text { years } \\
\text { Respondents } \\
\text { Parents and } \\
\text { recreation staff } \\
\text { Recruitment } \\
\text { Recreation } \\
\text { program } \\
\text { managers were } \\
\text { approached for } \\
\text { meetings and } \\
\text { a recruitment } \\
\text { package was } \\
\text { provided }\end{array}$ & $\begin{array}{l}14 \text { outdoor } \\
\text { recreation } \\
\text { ('Summer } \\
\text { Fun') sites in } \\
\text { Hawaii } \\
756 \text { parents } \\
\text { at baseline } \\
383 \text { parents } \\
\text { at post-test } \\
(50,6 \%) \\
285 \text { parents } \\
\text { at follow-up } \\
(37,1 \%)\end{array}$ & $\begin{array}{l}\text { Design } \\
\text { Three-arm rand- } \\
\text { omized trial- } \\
\text { Intervention } \\
\text { groups } \\
\text { 1. Control group } \\
\text { (110 parents) } \\
\text { 2. Education } \\
\text { intervention (122 } \\
\text { parents) } \\
\text { 3. Education and } \\
\text { environmental } \\
\text { intervention (53 } \\
\text { parents) } \\
\text { Duration } \\
\text { Three months } \\
\text { Baseline } \\
\text { Post-test, after } 6 \\
\text { weeks } \\
\text { Follow-up, after } \\
\text { three months } \\
\text { Randomization } \\
\text { A blocking strate- } \\
\text { gy was used with } \\
\text { balancing size } \\
\text { and location for } \\
\text { randomization }\end{array}$ & $\begin{array}{l}\text { Intervention type } \\
\text { Physical } \\
\text { Intervention level } \\
\text { Meso } \\
\text { The environmen- } \\
\text { tal component } \\
\text { consisted of on- } \\
\text { site sunscreen } \\
\text { dispensers, } \\
\text { portable shade } \\
\text { tents, posters } \\
\text { and policy con- } \\
\text { sultations } \\
\text { The control } \\
\text { group did not } \\
\text { receive an } \\
\text { intervention } \\
\text { component }\end{array}$ & $\begin{array}{l}\text { Sun safety } \\
\text { behaviours } \\
\text { (using } \\
\text { sunscreen, } \\
\text { wearing a } \\
\text { shirt with } \\
\text { sleeves, } \\
\text { wearing } \\
\text { sunglasses, } \\
\text { seeking } \\
\text { shade and } \\
\text { wearing a } \\
\text { hat). An av- } \\
\text { erage score } \\
\text { of these } \\
\text { behavioural } \\
\text { outcomes } \\
\text { was meas- } \\
\text { ured and } \\
\text { defined as a } \\
\text { 'sun-protec- } \\
\text { tion habit } \\
\text { index' }\end{array}$ & $\begin{array}{l}\text { Sun safety be- } \\
\text { haviours were } \\
\text { measured } \\
\text { with self-ad- } \\
\text { ministration } \\
\text { surveys for } \\
\text { parents and } \\
\text { monitoring } \\
\text { forms for rec- } \\
\text { reation staff } \\
\text { completed }\end{array}$ \\
\hline
\end{tabular}




\begin{tabular}{|c|c|c|c|c|c|c|c|}
\hline $\begin{array}{l}\text { Barankin et } \\
\text { al., } 2001\end{array}$ & Canada & $\begin{array}{l}\text { Target group } \\
\text { Children aged } \\
\text { between 9-10 } \\
\text { years } \\
\text { Respondents } \\
\text { Children, their } \\
\text { parents and } \\
\text { teachers } \\
\text { Recruitment } \\
\text { E-mails were } \\
\text { sent to all } \\
\text { public schools } \\
\text { in the Thames } \\
\text { Valley District } \\
\text { School Board }\end{array}$ & $\begin{array}{l}23 \text { Grade } 4 \\
\text { classes from } \\
16 \text { public } \\
\text { schools in } \\
\text { London, } \\
\text { Ontario, } \\
\text { Canada } \\
509 \text { children } \\
\text { at pre-test } \\
366 \text { children } \\
\text { at post-test } \\
\text { (71,9\%) } \\
259 \text { children } \\
\text { at follow-up } \\
(50,9 \%)\end{array}$ & $\begin{array}{l}\text { Design } \\
\text { Three-arm rand- } \\
\text { omized controlled } \\
\text { trial } \\
\text { Intervention } \\
\text { groups } \\
\text { 1. Control group } \\
\text { (97 children) } \\
\text { 2. Standard group } \\
\text { (107 children) } \\
\text { 3. Enhanced } \\
\text { group (55 chil- } \\
\text { dren) } \\
\text { Duration } \\
\text { Four months } \\
\text { Pre-test (May } \\
\text { 1999) } \\
\text { Post-test (June } \\
\text { 1999) } \\
\text { Follow-up (Sep- } \\
\text { tember 1999) } \\
\text { Randomization } \\
\text { Intervention } \\
\text { groups were } \\
\text { based on a first- } \\
\text { come-first-served } \\
\text { basis, according } \\
\text { to teachers' } \\
\text { response to } \\
\text { e-mails. The first } \\
\text { 16 schools who } \\
\text { responded were } \\
\text { randomized in the } \\
\text { two intervention } \\
\text { groups }\end{array}$ & $\begin{array}{l}\text { Intervention type } \\
\text { Economical } \\
\text { Intervention level } \\
\text { Meso } \\
\text { The environmen- } \\
\text { tal component } \\
\text { consisted of } \\
\text { provision of } \\
\text { sunscreen prior } \\
\text { to the summer } \\
\text { holiday in 1999, } \\
\text { combined with } \\
\text { information } \\
\text { sheets for } \\
\text { parents } \\
\text { Both the } \\
\text { standard and } \\
\text { enhanced group } \\
\text { received educa- } \\
\text { tional presenta- } \\
\text { tions about skin } \\
\text { cancer risk and } \\
\text { prevention at the } \\
\text { schools } \\
\text { The control } \\
\text { group received } \\
\text { activity books } \\
\text { with some sun } \\
\text { safety education }\end{array}$ & $\begin{array}{l}\text { Children's } \\
\text { attitudes } \\
\text { and aware- } \\
\text { ness about } \\
\text { conse- } \\
\text { quences of } \\
\text { excessive } \\
\text { sun exposure } \\
\text { and tanning, } \\
\text { children's } \\
\text { sun safety } \\
\text { behaviours } \\
\text { (using } \\
\text { sunscreen, } \\
\text { avoiding } \\
\text { midday } \\
\text { activities } \\
\text { and wearing } \\
\text { UV-protec- } \\
\text { tive clothing } \\
\text { and sun- } \\
\text { glasses) and } \\
\text { incidence } \\
\text { of children's } \\
\text { sunburns }\end{array}$ & $\begin{array}{l}\text { Children's } \\
\text { attitudes, } \\
\text { sun safety } \\
\text { behaviours } \\
\text { and sunburn } \\
\text { incidence was } \\
\text { measured } \\
\text { with surveys } \\
\text { for parents, } \\
\text { children and } \\
\text { teachers }\end{array}$ \\
\hline $\begin{array}{l}\text { Authors, } \\
\text { year }\end{array}$ & Country & $\begin{array}{l}\text { Target group, } \\
\text { recruitment }\end{array}$ & $\begin{array}{l}\text { Sample size } \\
\text { and setting }\end{array}$ & $\begin{array}{l}\text { Design (inter- } \\
\text { vention groups, } \\
\text { duration, ran- } \\
\text { domization) }\end{array}$ & Intervention & Outcomes & $\begin{array}{l}\text { Outcome } \\
\text { measure- } \\
\text { ments }\end{array}$ \\
\hline $\begin{array}{l}\text { Bauer et al., } \\
2005\end{array}$ & Germany & $\begin{array}{l}\text { Target group } \\
\text { Children aged } \\
\text { between 2-7 } \\
\text { years } \\
\text { Respondents } \\
\text { Children and } \\
\text { their parents } \\
\text { Recruitment } \\
\text { Public nursery } \\
\text { schools were } \\
\text { selected ran- } \\
\text { domly }\end{array}$ & $\begin{array}{l}78 \text { public } \\
\text { nursery } \\
\text { schools in } \\
\text { Stuttgart and } \\
\text { Bochum } \\
1887 \text { children } \\
\text { at baseline } \\
1232 \text { children } \\
\text { at follow-up } \\
\text { (68\%) }\end{array}$ & $\begin{array}{l}\text { Design } \\
\text { Randomized Con- } \\
\text { trolled Trial } \\
\text { Intervention } \\
\text { groups } \\
\text { 1. Control group } \\
\text { (398 children) } \\
\text { 2. Educational } \\
\text { group (369 chil- } \\
\text { dren) } \\
\text { 3. Education + } \\
\text { sunscreen group } \\
\text { (465 children) } \\
\text { Duration } \\
\text { Three years } \\
\text { Baseline assess- } \\
\text { ment (summer } \\
\text { 1998 in Stuttgart } \\
\text { and autumn } 1998 \\
\text { in Bochum) } \\
\text { Final assessment } \\
\text { (summer } 2001 \\
\text { in Stuttgart and } \\
\text { autumn } 2001 \text { in } \\
\text { Bochum) } \\
\text { Randomization } \\
\text { A random allo- } \\
\text { cation computer } \\
\text { program was used }\end{array}$ & $\begin{array}{l}\text { Intervention type } \\
\text { Economical } \\
\text { Intervention level } \\
\text { Meso } \\
\text { The environmen- } \\
\text { tal component } \\
\text { consisted of a } \\
\text { broad-spectrum } \\
\text { sunscreen bottle } \\
\text { (SPF 25) and } \\
\text { instructions on } \\
\text { sunscreen use, } \\
\text { which was pro- } \\
\text { vided to parents } \\
\text { yearly } \\
\text { Both the inter- } \\
\text { vention groups } \\
\text { received edu- } \\
\text { cational letters } \\
\text { (3 times a year) } \\
\text { with information } \\
\text { on sunscreen use } \\
\text { and melanoma } \\
\text { prevention } \\
\text { The control } \\
\text { group received } \\
\text { one educational } \\
\text { session prior to } \\
\text { the intervention } \\
\text { period }\end{array}$ & $\begin{array}{l}\text { The number } \\
\text { of nevi } \\
\text { incidence, } \\
\text { sun exposure } \\
\text { at home } \\
\text { and during } \\
\text { holidays, } \\
\text { sunburns, } \\
\text { sunscreen } \\
\text { use and } \\
\text { wearing } \\
\text { protective } \\
\text { clothing }\end{array}$ & $\begin{array}{l}\text { Nevi incidence } \\
\text { was measured } \\
\text { by physical } \\
\text { examina- } \\
\text { tion from } \\
\text { dermatolo- } \\
\text { gists, using a } \\
\text { standardized } \\
\text { protocol for } \\
\text { defining and } \\
\text { counting nevi } \\
\text { Children's } \\
\text { sun exposure, } \\
\text { history of } \\
\text { sunburns and } \\
\text { sunscreen use } \\
\text { was measured } \\
\text { with ques- } \\
\text { tionnaires for } \\
\text { parents. }\end{array}$ \\
\hline
\end{tabular}




\begin{tabular}{|c|c|c|c|c|c|c|c|}
\hline $\begin{array}{l}\text { Dobbinson } \\
\text { et al., } 2009\end{array}$ & Australia & $\begin{array}{l}\text { Target group } \\
\text { Adolescents, } \\
\text { aged between } \\
12-18 \text { years } \\
\text { Respondents } \\
\text { Adolescents } \\
\text { Recruitment } \\
\text { E-mails with } \\
\text { study aims and } \\
\text { requirements } \\
\text { were sent to } \\
\text { school princi- } \\
\text { pals }\end{array}$ & $\begin{array}{l}51 \text { Secondary } \\
\text { schools in } \\
\text { Melbourne } \\
\text { All schools } \\
\text { completed } \\
\text { the trial }\end{array}$ & $\begin{array}{l}\text { Design } \\
\text { Cluster Rand- } \\
\text { omized Controlled } \\
\text { Trial } \\
\text { Intervention } \\
\text { groups } \\
\text { 1. Control group } \\
\text { (26 schools) } \\
2 . \text { Intervention } \\
\text { group ( } 25 \text { schools) } \\
\text { Duration } \\
\text { Two years } \\
\text { Pre-test, before } \\
\text { installation } \\
\text { of shade sails } \\
\text { (2004/2005) } \\
\text { Post-test, after in- } \\
\text { stallation of shade } \\
\text { sails (2005/2006) } \\
\text { Randomization } \\
\text { A study statis- } \\
\text { tician randomly } \\
\text { assigned the } \\
\text { schools in groups }\end{array}$ & $\begin{array}{l}\text { Intervention type } \\
\text { Physical } \\
\text { Intervention level } \\
\text { Micro } \\
\text { The environmen- } \\
\text { tal component } \\
\text { consisted of } \\
\text { different sized } \\
\text { built shade sails } \\
\text { on school sites } \\
\text { The control } \\
\text { group did not } \\
\text { receive an } \\
\text { intervention } \\
\text { component }\end{array}$ & $\begin{array}{l}\text { The mean } \\
\text { number of } \\
\text { students } \\
\text { seeking } \\
\text { shade after } \\
\text { establishing } \\
\text { the shade } \\
\text { sails and the } \\
\text { mean num- } \\
\text { ber of stu- } \\
\text { dents using } \\
\text { alternative } \\
\text { sites (shade } \\
\text { avoidance) }\end{array}$ & $\begin{array}{l}\text { Shade use } \\
\text { was observed } \\
\text { by students } \\
\text { with digital } \\
\text { video cam- } \\
\text { eras and } \\
\text { reviewed } \\
\text { by research } \\
\text { assistants } \\
\text { following a } \\
\text { protocol }\end{array}$ \\
\hline $\begin{array}{l}\text { Harrison et } \\
\text { al., } 2010\end{array}$ & Australia & $\begin{array}{l}\text { Target group } \\
\text { Children, aged } \\
\text { between 0-35 } \\
\text { months } \\
\text { Respondents } \\
\text { Children } \\
\text { Recruitment } \\
\text { Unknown }\end{array}$ & $\begin{array}{l}25 \text { day-care } \\
\text { centres } \\
770 \text { children } \\
\text { at baseline } \\
\text { measure- } \\
\text { ment ( } 89 \% \\
\text { response) } \\
544 \text { children } \\
\text { at follow-up } \\
\text { (70,7\% re- } \\
\text { sponse) }\end{array}$ & $\begin{array}{l}\text { Design } \\
\text { Cluster Rand- } \\
\text { omized Controlled } \\
\text { Trial } \\
\text { Intervention } \\
\text { groups } \\
\text { 1. Control group } \\
\text { 2. Intervention } \\
\text { group } \\
\text { Duration } \\
\text { Three years } \\
\text { Baseline (Novem- } \\
\text { ber 1999) } \\
\text { Follow-up (2000, } \\
\text { 2001 and July } \\
\text { 2002) }\end{array}$ & $\begin{array}{l}\text { Intervention type } \\
\text { Economical } \\
\text { Intervention level } \\
\text { Micro } \\
\text { The environmen- } \\
\text { tal component } \\
\text { consisted of } \\
\text { provision of } \\
\text { sun-protective } \\
\text { clothing, hats } \\
\text { and swim shirts } \\
\text { for children in } \\
\text { the day-care } \\
\text { centres } \\
\text { The control } \\
\text { group did not } \\
\text { receive an } \\
\text { intervention } \\
\text { component }\end{array}$ & $\begin{array}{l}\text { The number } \\
\text { of nevi prev- } \\
\text { alence }\end{array}$ & $\begin{array}{l}\text { Nevi prev- } \\
\text { alence was } \\
\text { measured by } \\
\text { full-body skin } \\
\text { examinations }\end{array}$ \\
\hline
\end{tabular}




\begin{tabular}{|c|c|c|c|c|c|c|c|}
\hline $\begin{array}{l}\text { Dobbinson } \\
\text { et al., } 2019\end{array}$ & Australia & $\begin{array}{l}\text { Target groups } \\
\text { and respon- } \\
\text { dents } \\
\text { For observa- } \\
\text { tions: All park } \\
\text { visitors } \\
\text { For self-re- } \\
\text { port surveys: } \\
\text { Respondents } \\
\text { living nearby } \\
\text { the parks } \\
\text { For focus } \\
\text { groups: } \\
\text { Park visitors } \\
\text { aged >13 years } \\
\text { Recruitment } \\
\text { Local govern- } \\
\text { ment councils } \\
\text { were invited } \\
\text { with letters of } \\
\text { support, house- } \\
\text { holds received } \\
\text { surveys and } \\
\text { invitations for } \\
\text { focus groups } \\
\text { and in the } \\
\text { parks, signs } \\
\text { were displayed } \\
\text { to recruit } \\
\text { participants for } \\
\text { focus groups }\end{array}$ & $\begin{array}{l}6 \text { public } \\
\text { parks in } \\
\text { socioeco- } \\
\text { nomically } \\
\text { disadvan- } \\
\text { taged areas } \\
\text { in Melbourne }\end{array}$ & $\begin{array}{l}\text { Design } \\
\text { Non-randomized } \\
\text { pre-post con- } \\
\text { trolled trial } \\
\text { Intervention } \\
\text { groups } \\
\text { 1. Control parks } \\
\text { (no built shade) } \\
\text { 2. Intervention } \\
\text { parks (built } \\
\text { shade) } \\
\text { Duration } \\
\text { Three years } \\
\text { Pre-test (2013- } \\
\text { 2014) } \\
\text { Post-test (2014- } \\
\text { 2015) } \\
\text { Follow-up (2015- } \\
\text { 2016) } \\
\text { Randomization } \\
\text { Parks were } \\
\text { non-randomly se- } \\
\text { lected according } \\
\text { to existing refur- } \\
\text { bishment plans }\end{array}$ & $\begin{array}{l}\text { Intervention type } \\
\text { Physical } \\
\text { Intervention level } \\
\text { Macro } \\
\text { The environmen- } \\
\text { tal component } \\
\text { consisted of } \\
\text { built shade in } \\
\text { the intervention } \\
\text { parks. }\end{array}$ & Shade use & $\begin{array}{l}\text { Shade use } \\
\text { was measured } \\
\text { by observing } \\
\text { park users, } \\
\text { by self-report } \\
\text { surveys and } \\
\text { focus groups } \\
\text { with respon- } \\
\text { dents living } \\
\text { nearby the } \\
\text { parks }\end{array}$ \\
\hline
\end{tabular}


Table 3. Study outcomes

\begin{tabular}{|c|c|c|c|c|c|c|c|}
\hline $\begin{array}{l}\text { Authors, } \\
\text { year }\end{array}$ & Design & $\begin{array}{l}\text { Outcomes } \\
\text { related to } \\
\text { socio-cog- } \\
\text { nitive } \\
\text { determi- } \\
\text { nants }\end{array}$ & $\begin{array}{l}\text { Outcomes relat- } \\
\text { ed to sun safe } \\
\text { behaviour and } \\
\text { UVR exposure }\end{array}$ & $\begin{array}{l}\text { Outcomes } \\
\text { related to } \\
\text { sunburns/ } \\
\text { reported nevi }\end{array}$ & $\begin{array}{l}\text { Statistical } \\
\text { analyses }\end{array}$ & $\begin{array}{l}\text { Statistical } \\
\text { results }\end{array}$ & $\begin{array}{l}\text { Reported stand- } \\
\text { alone effects }\end{array}$ \\
\hline $\begin{array}{l}\text { Gallagh- } \\
\text { er et al., } \\
2000\end{array}$ & $\begin{array}{l}\text { Random- } \\
\text { ized Con- } \\
\text { trolled } \\
\text { Trial }\end{array}$ & N/A & $\begin{array}{l}\text { Parental } \\
\text { application of } \\
\text { broad spectrum } \\
\text { sunscreen (SPF } \\
\text { 30) on their child } \\
\text { and children's } \\
\text { UVR exposure }\end{array}$ & $\begin{array}{l}\text { Number of nevi } \\
\text { on the body } \\
\text { (left aside the } \\
\text { scalp, genital } \\
\text { areas and the } \\
\text { backside) }\end{array}$ & $\begin{array}{l}\text { Linear regres- } \\
\text { sion models } \\
\text { with number of } \\
\text { nevi as outcome } \\
\text { and various } \\
\text { single predictor } \\
\text { variables and } \\
\text { interaction } \\
\text { terms, using a } \\
\text { forward-selec- } \\
\text { tion algorithm } \\
(p<.10)\end{array}$ & $\begin{array}{l}\text { Sunscreen } \\
\text { use and UVR } \\
\text { exposure } \\
\text { No significant } \\
\text { differences in } \\
\text { sunscreen use } \\
\text { were found } \\
\text { Number of nevi } \\
\text { Children in the } \\
\text { intervention } \\
\text { group developed } \\
\text { significantly less } \\
\text { nevi (respec- } \\
\text { tively median } \\
\text { counts of } 24.0 \\
\text { and } 28.0, p= \\
\text { O48). The inter- } \\
\text { action between } \\
\text { randomization } \\
\text { to the interven- } \\
\text { tion group and } \\
\text { degree of nevi } \\
\text { was the stron- } \\
\text { gest statistical } \\
\text { predictor of } \\
\text { newly developed } \\
\text { nevi (Estimates } \\
\text { (SE); -0.38 (0.17), } \\
p=.03 \text { ) }\end{array}$ & $\begin{array}{l}\text { Sunscreen and UVR } \\
\text { exposure } \\
\text { Children were } \\
\text { equally protected by } \\
\text { sunscreen in the two } \\
\text { groups, with no sig- } \\
\text { nificant difference in } \\
\text { time spent outdoors } \\
\text { Number of nevi } \\
\text { Children from the } \\
\text { intervention group } \\
\text { developed signifi- } \\
\text { cantly less nevi at } \\
\text { the end of the study } \\
\text { period }\end{array}$ \\
\hline $\begin{array}{l}\text { Glanz et } \\
\text { al., } 2000\end{array}$ & $\begin{array}{l}\text { Three- } \\
\text { arm ran- } \\
\text { domized } \\
\text { trial }\end{array}$ & N/A & $\begin{array}{l}\text { Children's own } \\
\text { sun protection } \\
\text { behaviours, } \\
\text { defined as a sun } \\
\text { protection habit } \\
\text { index: } \\
\text { - Wearing a shirt } \\
\text { with sleeves } \\
\text { - Wearing sun- } \\
\text { glasses } \\
\text { - Seeking shade } \\
\text { - Wearing a hat } \\
\text { - Use of sun- } \\
\text { screen }\end{array}$ & N/A & $\begin{array}{l}\text { Mixed model } \\
\text { analyses of } \\
\text { variance, ANOVA }\end{array}$ & $\begin{array}{l}\text { Sun safe be- } \\
\text { haviours } \\
\text { Sun protection } \\
\text { habit index } \\
\text { increased in the } \\
\text { education ( } 0.20 \\
\text { and } p<.001) \\
\text { and education } \\
+ \text { environmental } \\
\text { intervention ( } 0.19 \\
\text { and } p<.001) \\
\text { compared to the } \\
\text { control group } \\
\text { (.06), whereas } \\
\text { solely sunscreen } \\
\text { use increased in } \\
\text { the education } \\
\text { intervention } \\
\text { group only (0.16 } \\
\pm 0.08 \text { and } p< \\
\text { O.05). } \\
\text { Other be- } \\
\text { haviours } \\
\text { No significant } \\
\text { differences were } \\
\text { found }\end{array}$ & $\begin{array}{l}\text { No significant differ- } \\
\text { ences in outcomes } \\
\text { were found between } \\
\text { the education and } \\
\text { education + environ- } \\
\text { mental intervention }\end{array}$ \\
\hline $\begin{array}{l}\text { Authors, } \\
\text { year }\end{array}$ & Design & $\begin{array}{l}\text { Outcomes } \\
\text { related to } \\
\text { socio-cog- } \\
\text { nitive } \\
\text { determi- } \\
\text { nants }\end{array}$ & $\begin{array}{l}\text { Outcomes relat- } \\
\text { ed to sun safe } \\
\text { behaviour and } \\
\text { UVR exposure }\end{array}$ & $\begin{array}{l}\text { Outcomes } \\
\text { related to } \\
\text { sunburns/ } \\
\text { reported nevi }\end{array}$ & $\begin{array}{l}\text { Statistical } \\
\text { analyses }\end{array}$ & $\begin{array}{l}\text { Statistical } \\
\text { results }\end{array}$ & $\begin{array}{l}\text { Reported stand- } \\
\text { alone effects }\end{array}$ \\
\hline
\end{tabular}




\begin{tabular}{|c|c|c|c|c|c|c|c|}
\hline $\begin{array}{l}\text { Ba- } \\
\text { rankin et } \\
\text { al., } 2001\end{array}$ & $\begin{array}{l}\text { Three- } \\
\text { arm Ran- } \\
\text { domized } \\
\text { Con- } \\
\text { trolled } \\
\text { trial }\end{array}$ & $\begin{array}{l}\text { Children's } \\
\text { attitudes } \\
\text { about tan- } \\
\text { ning and } \\
\text { awareness } \\
\text { about con- } \\
\text { sequences } \\
\text { of exces- } \\
\text { sive sun } \\
\text { exposure } \\
\text { Teacher's } \\
\text { estima- } \\
\text { tion of } \\
\text { children's } \\
\text { awareness } \\
\text { of conse- } \\
\text { quences of } \\
\text { UVR }\end{array}$ & $\begin{array}{l}\text { Children's own } \\
\text { sunscreen ap- } \\
\text { pliance and pa- } \\
\text { rental sunscreen } \\
\text { application } \\
\text { (15-30 minutes } \\
\text { prior to going } \\
\text { out in the sun, } \\
\text { reapplication), } \\
\text { avoidance of } \\
\text { midday activities, } \\
\text { wearing long } \\
\text { sleeved shirts } \\
\text { and long pants } \\
\text { and sunglasses }\end{array}$ & $\begin{array}{l}\text { Number of } \\
\text { sunburns in } \\
\text { children }\end{array}$ & Missing data & $\begin{array}{l}\text { Children } \\
\text { The enhanced } \\
\text { group showed } \\
\text { the greatest } \\
\text { reduction ( } p< \\
\text {.05) in children's } \\
\text { attitude favour- } \\
\text { ing tanning. } \\
\text { No significant } \\
\text { differences in } \\
\text { other outcomes } \\
\text { were found } \\
\text { Parents } \\
\text { No significant } \\
\text { differences were } \\
\text { found } \\
\text { Teachers } \\
\text { No statistical } \\
\text { results were } \\
\text { mentioned }\end{array}$ & $\begin{array}{l}\text { Children in the en- } \\
\text { hanced intervention } \\
\text { group had signifi- } \\
\text { cantly the greatest } \\
\text { decrease in tanning } \\
\text { favouring attitudes } \\
\text { compared to the } \\
\text { other groups }\end{array}$ \\
\hline $\begin{array}{l}\text { Bauer et } \\
\text { al., } 2005\end{array}$ & $\begin{array}{l}\text { Random- } \\
\text { ized Con- } \\
\text { trolled } \\
\text { Trial }\end{array}$ & N/A & $\begin{array}{l}\text { Parental applica- } \\
\text { tion of sunscreen } \\
\text { and putting on } \\
\text { protective cloth- } \\
\text { ing and children's } \\
\text { UVR exposure }\end{array}$ & $\begin{array}{l}\text { Newly devel- } \\
\text { oped mela- } \\
\text { nocytic nevi } \\
\text { and sunburn } \\
\text { incidence }\end{array}$ & $\begin{array}{l}\text { Chi-Squared } \\
\text { tests, analyses } \\
\text { of variance and } \\
\text { nonparametric } \\
\text { Kruskal-Wallis } \\
\text { tests were con- } \\
\text { ducted to test } \\
\text { for differences } \\
\text { between control } \\
\text { and interven- } \\
\text { tion groups. } \\
\text { Wilcoxon tests, } \\
\text { Chi squared test } \\
\text { statistics and } \\
\text { Fisher's exact } \\
\text { test were con- } \\
\text { ducted to study } \\
\text { two groups at } \\
\text { one time }\end{array}$ & $\begin{array}{l}\text { Sunscreen use } \\
\text { There were } \\
\text { group differenc- } \\
\text { es in children's } \\
\text { sunscreen use ( } p \\
=.03 \text { ), however } \\
\text { not present } \\
\text { between the } \\
\text { two intervention } \\
\text { groups } \\
\text { Protective } \\
\text { clothing } \\
\text { No significant } \\
\text { differences were } \\
\text { found } \\
\text { Nevi } \\
\text { No significant } \\
\text { differences were } \\
\text { found } \\
\text { Spent holidays } \\
\text { There were } \\
\text { group differ- } \\
\text { ences in weeks } \\
\text { spent on holi- } \\
\text { days ( } p=.02 \text { ), } \\
\text { and in holidays } \\
\text { spent further } \\
\text { away from the } \\
\text { equator ( } p= \\
\text {.oog) }\end{array}$ & $\begin{array}{l}\text { Children in the } \\
\text { education + sun- } \\
\text { screen group did } \\
\text { not use sunscreen } \\
\text { nor wore protective } \\
\text { clothing more often } \\
\text { than children in the } \\
\text { other groups. Also, } \\
\text { no differences in } \\
\text { development of nevi } \\
\text { were found } \\
\text { Respondents in the } \\
\text { environmental inter- } \\
\text { vention group signifi- } \\
\text { cantly reported lower } \\
\text { median numbers } \\
\text { of weeks spent on } \\
\text { holidays in sunny } \\
\text { climates. However, } \\
\text { respondents in this } \\
\text { group also reported } \\
\text { to go on holidays } \\
\text { further away from } \\
\text { the equator than } \\
\text { respondents in the } \\
\text { control group }\end{array}$ \\
\hline
\end{tabular}




\begin{tabular}{|c|c|c|c|c|c|c|c|}
\hline $\begin{array}{l}\text { Dobbin- } \\
\text { son et } \\
\text { al., } 2009\end{array}$ & $\begin{array}{l}\text { Cluster } \\
\text { Random- } \\
\text { ized Con- } \\
\text { trolled } \\
\text { Trial }\end{array}$ & N/A & $\begin{array}{l}\text { Usage of shaded } \\
\text { areas and usage } \\
\text { of alternative } \\
\text { sites }\end{array}$ & $\mathrm{N} / \mathrm{A}$ & $\begin{array}{l}\text { Differences in } \\
\text { aggregated } \\
\text { shade use } \\
\text { (mean value) be- } \\
\text { tween pre-test } \\
\text { and post-test in } \\
\text { both conditions } \\
\text { were studies } \\
\text { with unpaired } \\
\text { t-tests. General- } \\
\text { ized estimating } \\
\text { equations with } \\
\text { robust standard } \\
\text { errors were } \\
\text { fitted to the } \\
\text { data to test } \\
\text { for interaction } \\
\text { between specific } \\
\text { school differ- } \\
\text { ences and sites. } \\
\text { Non-aggregated } \\
\text { data were used } \\
\text { in linear mixed } \\
\text { models to test } \\
\text { for intra school } \\
\text { correlation } \\
\text { coefficients }\end{array}$ & $\begin{array}{l}\frac{\text { Shade use }}{\text { The mean }} \text { change in use } \\
\text { of sites between } \\
\text { pre-test and } \\
\text { post-test was } \\
\text { higher in the } \\
\text { intervention } \\
\text { than the control } \\
\text { (mean change } \\
\text { of 2.67 and -.03, } \\
p=.011 \text { ) group } \\
\text { Shade avoid- } \\
\text { ance } \\
\text { The mean } \\
\text { change in using } \\
\text { different sites in } \\
\text { the interven- } \\
\text { tion group was } \\
\text { greater for the } \\
\text { shaded areas } \\
\text { than the alter- } \\
\text { native sites (dif- } \\
\text { ference in mean } \\
\text { change between } \\
\text { sites } 2.70, \mathrm{p}= \\
\text {.007). At the } \\
\text { control schools, } \\
\text { no significant } \\
\text { differences were } \\
\text { found }\end{array}$ & $\begin{array}{l}\text { Adolescent active } \\
\text { use of purpose built } \\
\text { shade increased } \\
\text { at the intervention } \\
\text { schools }\end{array}$ \\
\hline $\begin{array}{l}\text { Harrison } \\
\text { et al., } \\
2010\end{array}$ & $\begin{array}{l}\text { Cluster } \\
\text { Random- } \\
\text { ized Con- } \\
\text { trolled } \\
\text { Trial }\end{array}$ & N/A & N/A & $\begin{array}{l}\text { Incidence of } \\
\text { pigmented } \\
\text { moles }\end{array}$ & $\begin{array}{l}\text { Missing data: } \\
\text { conference } \\
\text { paper }\end{array}$ & $\begin{array}{l}\text { The median } \\
\text { count of incident } \\
\text { moles was high- } \\
\text { er in the control } \\
\text { than the inter- } \\
\text { vention group } \\
\text { (respectively } \\
16 \text {; range } 0-77 \\
\text { versus } 12,5 \text {; and } \\
\text { O- } 74, \mathrm{p}=.02 \text { ). } \\
\text { The median in- } \\
\text { cidence of moles } \\
\text { per month was } \\
\text { also higher in } \\
\text { the control than } \\
\text { the intervention } \\
\text { group (respec- } \\
\text { tively } 0.68 \text { and } \\
0.46, p=.001 \text { ) }\end{array}$ & $\begin{array}{l}\text { There was signifi- } \\
\text { cantly less pigment- } \\
\text { ed mole incidence } \\
\text { in the intervention } \\
\text { group, compared to } \\
\text { the control group }\end{array}$ \\
\hline $\begin{array}{l}\text { Dobbin- } \\
\text { son et } \\
\text { al., } 2019\end{array}$ & $\begin{array}{l}\text { Non-ran- } \\
\text { domized } \\
\text { pre-post } \\
\text { con- } \\
\text { trolled } \\
\text { trial }\end{array}$ & N/A & $\begin{array}{l}\text { Usage of shaded } \\
\text { areas }\end{array}$ & N/A & $\begin{array}{l}\text { Missing data: } \\
\text { conference } \\
\text { paper }\end{array}$ & $\begin{array}{l}\text { Intervention-re- } \\
\text { ceived analyses } \\
\text { showed in- } \\
\text { creased shade } \\
\text { use by visitors } \\
(p=.04)\end{array}$ & $\begin{array}{l}\text { Significantly more } \\
\text { people used shade } \\
\text { at follow-up at the } \\
\text { intervention parks } \\
\text { compared to the } \\
\text { control parks }\end{array}$ \\
\hline
\end{tabular}




\section{Appendix A \\ Examples of search strings}

\section{A1. PubMed}

"Child, Preschool"(Mesh) OR "Child"(Mesh) OR "Adolescent"(Mesh) OR "Infant"(Mesh) OR Child(tiab) OR Preschool(tiab) OR Children(tiab) OR schoolgirl(tiab) OR schoolboy(tiab) OR Adolescent(tiab) OR Adolescents(tiab) OR Youngster(tiab) OR Youngsters(tiab) OR Youth(tiab) OR Minors(tiab) OR Boys(tiab) OR Girls(tiab)

AND

Protection(tiab) OR Preventing(tiab) OR Prevention(tiab) OR "prevention and control"(Subheading) OR "prevention and control"(tiab) OR Intervention(tiab) OR "Health promotion"(tiab) OR "Health promotion"(Mesh) OR "Environment design"(Mesh) OR "Environment design"(tiab) OR "Environment and public health"(tiab) OR "Environmental exposure"(Mesh) OR "physical environment"(tiab) OR "urban design"(tiab) OR "built environment"(tiab) OR "shade"(tiab) OR "shade provision"(tiab) OR "architecture"(tiab)

AND

Outdoor(tiab) OR Outside(tiab) OR "swimming pools"(Mesh) OR "Swimming pool"(tiab) OR "Swimming pools"(tiab) OR "Recreation area"(tiab) OR "Recreation areas"(tiab) OR "Recreational area"(tiab) OR "Recreational areas"(tiab) OR "Recreation place"(tiab) OR "Recreation places"(tiab) OR "Recreational places"(tiab) OR "open air"(tiab) OR Schools(tiab) OR "Schools"(Mesh) OR "Child Day Care Centers"(tiab) OR "Child Day Care Centers"(Mesh) OR Parks(tiab) OR Vacationing(tiab) OR play(Mesh) OR plaything(tiab)

AND

("Sunburn"(Mesh) OR "Sun burning"(tiab) OR Sunburn(tiab) OR Sunburns(tiab) OR Sunburning(tiab)) OR ((Sunlight/adverse effects(Mesh) OR Sunshine(tiab) OR Sun(tiab) OR "Sunbathing"(Mesh) OR Sunbathing(tiab) OR Suntanning(tiab) OR "Ultraviolet Rays"(Mesh) OR "UV radiation" (tiab) OR UVR(tiab) OR "Ultra Violet Rays" (tiab) OR "Ultra-Violet Ray" (tiab) OR "UV Light"(tiab) OR UVL(tiab) OR "Ultraviolet Ray" (tiab) OR "Ultraviolet Light"(tiab)) AND (burn(tiab) OR burning(tiab) OR Reddening(tiab) OR overexposure(tiab) OR Exposure(tiab) OR Burden(tiab) OR "skin neoplasms"(Mesh) OR "Skin Neoplasm"(tiab) OR "Skin Neoplasms"(tiab) OR "Skin Cancer" (tiab) OR "Skin Cancers"(tiab))) OR "Melanoma"(Mesh) OR "Sun Safety"(tiab) OR "Sun-safety"(tiab) OR "Sun safe"(tiab) OR "Sun-safe"(tiab)

AND

"Cluster randomized Trial"(tiab) OR "Field trials"(tiab) OR "Observational Study"(Publication Type) OR "Experimental design"(tiab) OR "Randomized Controlled Trial"(Publication Type) OR ((random*(tiab) AND (controlled(tiab) OR control(tiab) OR placebo(tiab) OR versus(tiab) OR vs(tiab) OR group(tiab) OR groups(tiab) OR comparison(tiab) OR compared(tiab) OR crossover(tiab) OR cross-over(tiab)) AND (trial(tiab) OR study(tiab))) OR ((single(tiab) OR double(tiab) OR triple(tiab)) AND (masked(tiab) OR blind*(tiab)))) 


\section{A.2. Google Scholar}

Infants OR children OR adolescents

AND

Protection OR prevention OR "prevention and control" OR intervention OR "health promotion" OR "environment design" OR "environment and public health" OR "built environment" OR shade OR "environmental exposure" OR "purpose built" OR "shade provision" OR "urban design" OR "physical environment" OR "urban architecture"

AND

Sunburn OR sunlight OR sunshine OR sun OR sunbathing OR "sun exposure" OR "UV radiation" OR UVR OR "overexposure" OR "skin cancer" OR melanoma OR "sun safe"

AND

Outdoor OR outside OR "swimming pool" OR "recreational area" OR recreation OR "recreational place" OR "Open air" OR school OR playground OR "child day care center" OR park OR vacationing OR play OR plaything

AND

RCT OR "randomized controlled trial" OR "observational study" OR "cluster randomized trial" OR "experimental study" OR "quasi experiment" OR "experimental design" OR "group comparison" OR "control group" 


\section{Appendix B \\ Flow diagram of included papers}

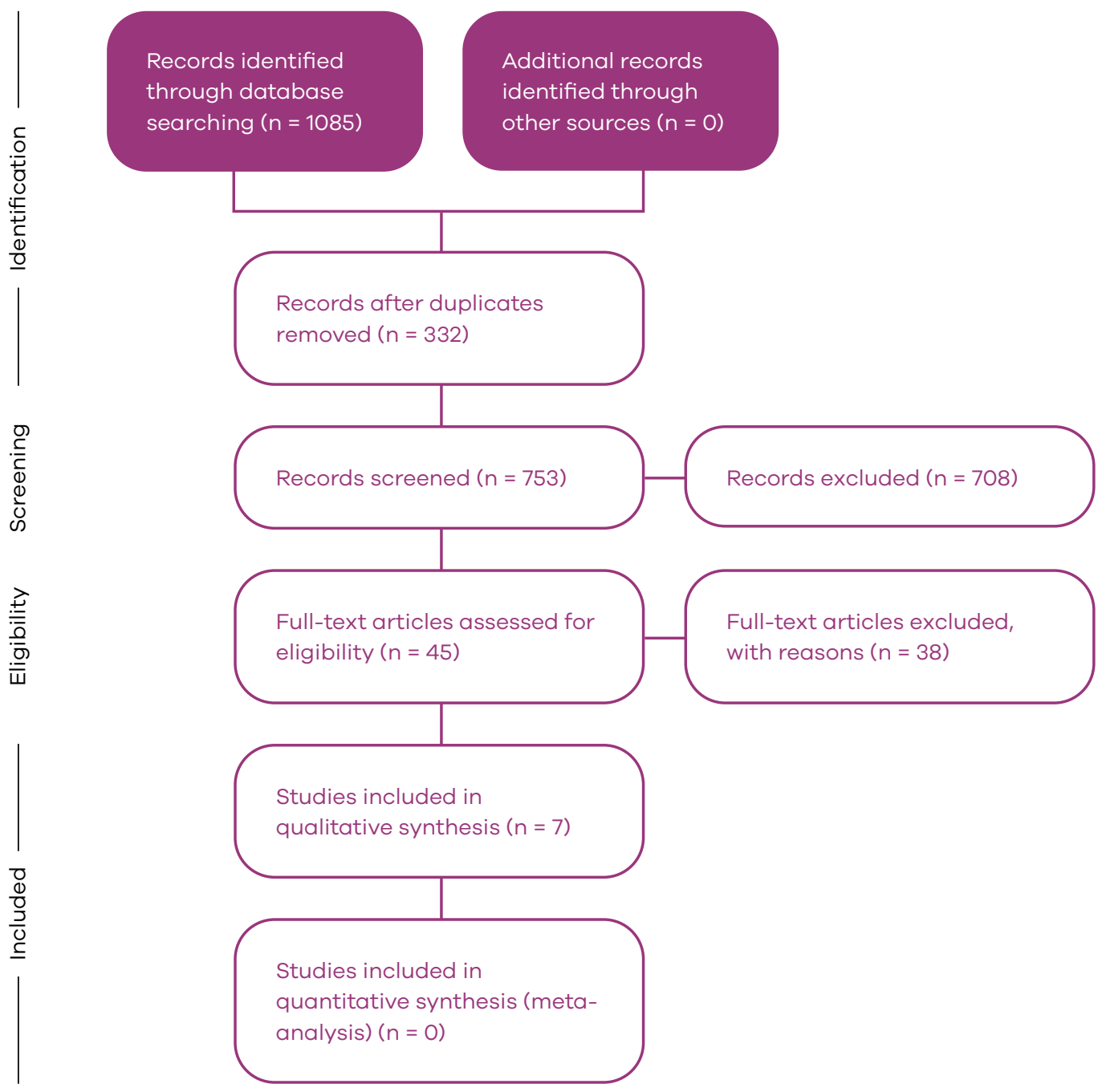


chapter seven

\section{General discussion}


'Between stimulus and response there is a space.

In that space is our power to choose our response. In our response lies our growth and our freedom.'

Viktor E. Frenkl 
The main aim of this thesis was to expand current insight into determinants of sunburn and sun protection behaviours for children aged between 4 and 12 years old. With this aim, we investigated parental and children's factors related to sunburn and sun protection and explored the influence of environmental factors. By increasing understanding of parental sun protection behaviours as well as the interaction between parents' and their children's behaviours, recommendations for optimizing skin cancer prevention interventions can be suggested. This final Chapter first discusses all findings concerning sunburn (Part 1.1), performance of sun protection behaviours (Part 1.2) and understanding of sun protection behaviours (Part 1.3), as outlined in this thesis. Next, limitations and strengths of the conducted studies are provided (Part 2), followed by conclusions and implications for future research and intervention development for skin cancer prevention (Part 3).

\section{Part I. Summary of the main findings}

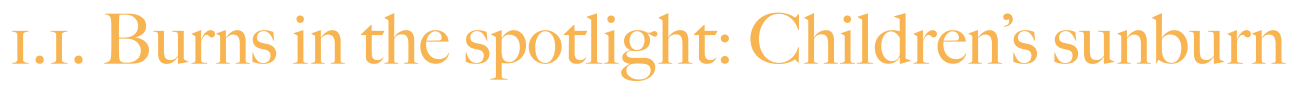

Since insight in sunburn prevalence among Dutch children has been limited up to now, we aimed to explore and understand children's sunburn experiences. Our data described in Chapters 2, 3 and 5 revealed that: 1) despite the awareness of necessary sun protection measures and fair sun protection behaviours among the majority of parents, children's sunburn prevalence was high and some children's characteristics appeared to increase their sunburn risk; 2) high-risk situations for inadequate sun protection behaviours and children's sunburn were often water-related; 3 ) conceptualization of sunburn seemed difficult for parents; and 4) sunburn did not seem to be a cue to action for parental sun protection behaviours.

\section{Sunburn prevalence}

Both a quantitative study among 670 parents (Chapter 3 ) and a qualitative interview study among 26 parents (Chapter 2) indicated that more than $75 \%$ of children experienced sunburn throughout their lives at least once. Furthermore, almost all parents in the interview study recalled a sunburn throughout their children's life, despite appearing highly aware and knowledgeable of the need for sun protection behaviours and reporting performance of at least one sun protection measure. Based on parental proxy-reports, over $42 \%$ of children experienced at least one sunburn in the foregoing year. The sunburn prevalence among Dutch children between studies in this thesis showed percentages that were comparable to or higher than in international observations $(48,50-52,154)$. Older children ( $\geq 10$ years) seemed more at-risk for experiencing sunburn than children of younger age ( 4 to 6 years) as outlined in Chapter 5 , which is consistent with previous international studies $(50,247)$. Since sunburn is indicative of insufficient sun protection behaviour (372), and sunburn during early life increases the risk of developing skin cancer profoundly $(4,41,42,45,282)$, these numbers underline the necessity for preventative efforts in the Netherlands.

\section{Situations with increased risk for sunburn}

Although results from our studies, as described in Chapters 4 and 5 , indicated that sun protection behaviours in incidental sun exposure situations (e.g. when cycling or playing) were less frequently performed by parents, we also noticed that planned sun exposure situations, often water-related (e.g. going to a lake, the beach or a swimming pool), increased the risk for sunburn, as was previously documented as well (80). 
Firstly, as outlined in Chapter 2, parents perceived these situations involving water-related activities as especially difficult since children were more hesitant to be protected with sunscreen or clothing, sunscreen was not water-resistant and therefore needed to be reapplied more often, or shade was unavailable. This finding is in line with previous studies that found that children's sun protection was largely insufficient in beach settings, although recent data is limited. In these settings, parents did not put on T-shirts, sunglasses or hats, relied solely on sunscreen, or their children were playing in unshaded areas $(78,289,373,374)$.

Secondly, the finding that water-related sun exposure seems to relate to sunburn risk was reflected in our quantitative study, as sun exposure in such planned exposure situations seemed to relate to increased sunburn risk. That is, children whose sun exposure in these beach or swimming pool settings was limited, appeared to have a lower risk of sunburn (see Chapter 5).

Thirdly, our results further indicated that children of parents reporting frequent sun exposure combined with high levels of sun protection behaviours in these situations, were still at risk for experiencing sunburn, as also described in Chapter 5. Overall, two main factors underlying an increased risk for inadequate protection in these water-related situations became apparent; 1) Environmental characteristics (e.g. lack of shade, water causing ineffective protection by sunscreen) may impede adequate parental sun protection behaviours, and 2) There may be a discrepancy between parents' subjective reports and objective performance of their sun protection behaviours, i.e. parents may inadequately perform sun protection strategies in these situations, even though they consider themselves as adhering to sun protection guidelines. Together, these results imply that planned sun exposure situations signify high risk for sunburn, which makes adequate sun protection strategies imperative. Environmental adaptations (e.g. shade provision) could facilitate parents to overcome barriers in these water-related situations. Moreover, educational efforts to encourage sun avoidance in these risky situations deserves closer attention in order to prevent children's sunburn as well.

\section{It's not (just) a burning memory}

With regard to parental reports of their children's experienced sunburn, two interesting findings were revealed, as described in Chapters 2, 3 and 5.

Firstly, we found a notable variability in reported sunburn rates among children between previous studies (ranging from $28 \%$ (154) to $73 \%$ (53) in the previous year), our own quantitative studies (ranging from almost $30 \%$ in the previous summer season, to $42 \%$ in the foregoing year and almost $78 \%$ throughout their lives) and our qualitative study, in which almost all children experienced sunburn in their lives. These differences could indicate that a possible reporting bias might be at play, since previous research described that proxyreports of sunburn may decrease validity of sunburn estimations $(198,375)$. Differences in recall periods or approaches of assessing sunburn frequency could also explain this variation (198).

Secondly, as described in Chapter 2, we found an initial underreporting of children's sunburn. Parents seemed reluctant to talk about their children's sunburn when a history of sunburn was asked for, but ultimately reported high frequencies. Sunburn can be regarded as a 'relatively memorable and distinct event' (Shoveller et al., 2001; p. 85) (198). With this in mind, one might expect parents to vividly recall their children's sunburn, so the question arises what factors were underlying of the initial underreporting of sunburn. One possibility could be that the parents in our sample originally provided socially desirable answers $(50,52,204)$ since they may feel criticized, making them hesitant to report sunburn. It was previously described that reporting sunburn may reflect parental feelings of their deficient protection efforts (376). Another reason could be that parents do not recognize sunburn as such. For example, a minor sunburn (e.g. without painful skin, or on smaller parts of the body) may not be identified as sunburn among parents (50), a finding that also seemed apparent in our qualitative study. The interpretation of sunburn was not further assessed in our survey study - in which reported prevalence of sunburn was lower, but we can assume that parental misconceptions about what is considered a sunburn was also apparent. With this in mind, sunburn percentages reported in the quantitative study may be expected to be higher in reality, which 


\section{Sunburn: a cue to action for parents?}

As outlined in Chapter 3, children's previous sunburn experiences did not result in better parental sun protection behaviours. International studies monitoring population sunburn prevalence demonstrate stable or increased sunburn rates over the years among adolescents and adults $(49,216,377)$, indicating that previous experienced sunburns increase the risk of subsequent sunburns. Our finding implies that previous sunburn among children did not improve parental sun protection behaviours and therefore did not function as a cue to action for parents to enhance future sun protection strategies (378). Currently, there are no studies that systematically investigate the role of sunburn as a possible cue to action (101). The possible explanations for our finding are threefold.

Firstly, a behavioural pattern might be at play, in which inadequate parental sun protection behaviour in the past results in a habitual form of inadequate behaviour and consequently causes higher sunburn rates among children. A recent meta-analysis regarding various parent-for-child health behaviours suggested that past behaviours strongly influence current behaviour, supporting the important role of habit (237). With regard to explaining parental sun protection behaviours specifically, parental habit also was of significant influence in another study (131).

Secondly, an appearance-based ideal of a tanned skin might outweigh the perceived harm of sunburn. As described in Chapters 2 and 3, we found a positive attitude towards children's tanned skin among parents, which also appeared to be confirmed by important peers. It has been previously described that a positive attitude about a tanned appearance can have a negative effect on parental sun protection behaviours ( 51 , $52,161)$. Although we did not investigate this relation explicitly, we indicated that a positive attitude among our samples of parents resulted in less optimal sun protection behaviours.

Thirdly, although parents in our sample seemed highly aware of the need for sun protection, they may underrate or be unaware of the risk of sunburn in childhood, therefore failing to improve their sun protection behaviours for their children after a sunburn experience. Several reasons for this have been documented. In a systematic review, misconceptions about sun exposure risk were detected among various populations, such as believing that sun exposure leads to increased resistance of the skin (101). Furthermore, an ageing skin rather than sunburn was most often mentioned as negative consequence of sun exposure. The notion that the negative consequences of sunburn is only present in the far future, could also cause inadequate sun protection behaviours (379). Among parents specifically, low perceptions of their child's skin cancer risk were associated with more sunburns (50). In our studies, misconceptions regarding sunburn could also have been at play among the parents. Overall, further examination of long-term behavioural patterns and the role of (repeated) sunburn is recommended.

\section{I.2. There is no perfect, but there are many good ways: Performance of sun protection behaviours}

From our investigation of the performance of several parent-for-child and children's own sun protection behaviours, three main findings emerged. First, although the performance of protective behaviours was fair, we revealed significant room for improvement, especially regarding seeking shade and clothing behaviour during incidental sun exposure. Moreover, parents performed sun protection behaviours more often in planned (e.g. going to the beach) than incidental (e.g. cycling) sun exposure situations and most often for younger children with more sensitive skin types. Second, we found that whereas direct sun protection behaviours declined, indirect sun protection behaviours (i.e. supporting children in performing sun protection behaviours themselves) remained relatively stable as children's age increased. Third, our 
analyses indicated that children themselves are executing sun protection behaviours, and that especially from the age of 11 and onwards they increasingly perform sun safety strategies more often than their parents do for them.

\section{Parent-for-child sun protection behaviours}

Several important findings emerged with regard to different types of sun protection behaviours performed by the parents in our studies.

Firstly, parents most frequently reported sunscreen use as their main protection behaviour, as was reported in Chapters 2, 4 and 5. This preference for the use of sunscreen is also reflected in parental sun protection behaviours reported in systematic reviews $(79,120)$. Although parents seemed to prefer the use of sunscreen as protection method, they also indicated that adequate appliance of sunscreen was complicated for them (see Chapter 2), which was described in previous work as well $(53,163)$. Confusion about the required SPF, questioning the water resistance of sunscreen, doubting when to reapply sunscreen and forgetting to apply sunscreen prior to going outdoors were often mentioned as factors contributing to the perceived difficulty of sunscreen use, as described in Chapter 2. Since adequate effectiveness of sunscreen can only be achieved by correct application and reapplication (297), the need for accurate knowledge on adequate application became apparent.

Secondly, clothing and especially seeking shade behaviours were less often reported. Even though the majority of parents who were interviewed were aware of the availability of UV-protective clothing and were in favour of seeking shade (described in Chapter 2), they most often applied sunscreen. Parents reported barriers concerning performance of clothing and seeking shade, e.g. forgetting to put on clothing or seek shade, clothing being uncomfortable for children, or shade being unavailable at outdoor venues, which has been previously described as well (53). According to international guidelines, sunscreen is not regarded as sufficient when used as the only method, and should be performed in conjunction with other sun protection behaviours (6). More importantly, since clothing and shade-seeking behaviours are highly recommended to protect children's skin $(72,73)$, and are regarded as more effective in reducing sunburn among children than sunscreen only $(50,79,82)$, promotion of these behaviours among parents is warranted.

Thirdly, as noted earlier, parents in our studies reported sun protection behaviours more frequently in planned compared to incidental sun exposure situations, as described in Chapters 4 and 5 . This difference could be explained by parents underestimating the risk of sun exposure in everyday settings and consequently underestimating the need for protection strategies, which has been reported before ( 15 , 40). Moreover, it was previously documented that parents seem to apply sunscreen more often in a beach rather than everyday setting $(263,380)$.

A systematic review revealed that people generally perceive 'a holiday' setting, such as the beach, as a risk situation for skin cancer development, not sufficiently realizing that incidental exposure - practically involving all other types of sun exposure, is associated with this as well (101). These and our findings emphasize the need for differentiating sun protection behaviours and sun exposure for various situations and when performing different activities $(119,157,193)$. Comparing sun protection behaviours in settings where sun exposure is intended versus situations in which sun exposure is acquired in an everyday setting, has received little attention in parental-focused skin cancer prevention studies thus far $(289,313)$. This is remarkable, since different types of acquired UVR-exposure, i.e. both intermittent and total accumulative received exposure, are associated with skin cancer development (381).

Fourthly, several characteristics of the child were shown to be related to parental sun protection behaviours. First of all, parents reported less sun protection behaviours for older children, as outlined in Chapters 4 and 5. The finding that parents seem to be less vigilant towards older children is in line with a consistent pattern across previous studies $(54,77,79,289)$. Second, our analyses revealed differences in sun protection behaviours across children's skin types. Parents more often reported protective measures for children 
with a more sensitive skin. Since skin sensitivity is related to increased skin cancer risk, these findings are promising and indicative of parents' awareness of their children's sun-sensitivity, which is also consistent with earlier work $(50,53,54)$. However, the need for adequate sun protection remains important for children with less sensitive skin types since skin cancer does occur among people with darker complexions, among which higher mortality rates have been reported (382).

Fifthly, with regard to parental characteristics influencing their sun protection behaviours, in all studies no differences in parental sun protection performance across children's sex were found. These findings are different from some other studies indicating that parents perform different types of sun protection measures for boys and girls, e.g. girls being more often protected with sunscreen (54) and hats, and boys with clothes $(76,373)$. Our finding seems encouraging as well, since previous studies have also reported that boys appear to be at greater risk for sunburn $(76,260,383)$.

Furthermore, while one's socioeconomic status (SES) has regularly been demonstrated to be positively related to health behaviours and outcomes (384-387), we did not detect such pattern in parental sun protection behaviours across the conducted studies. Although a positive association between educational level and sun protection behaviours seems apparent among adult populations (379), studies investigating parental education level and sun protection behaviours for their children demonstrate variable results, in which none $(388,389)$, inconsistent $(76,119)$ or minor $(390)$ differences in parental sun protection were revealed. These findings may implicate that parent-for-child sun protection behaviours were not influenced by educational attainment by the parents from our study sample, which is encouraging.

\section{Parents' indirect sun protection behaviours}

Findings in this thesis demonstrated that supportive behaviour, in which parents encourage children to perform their own sun protection behaviours, was relatively high across children's age and sex. Since parental involvement is regarded highly important for teaching children to perform sun protection behaviours on their own (48, 391), our findings seem promising. Since children's own sun protection behaviours at later ages seem to be influenced by parental attitudes and behaviours $(51,52,79,391)$ the supportive role of parents in stimulating and teaching children to perform their own sun protection behaviours as they get older, may deserve extra focus in sun safety interventions. Further, as our results showed that supportive behaviours appeared relatively stable across children's age and sex, performance of these behaviours could potentially be driven by other factors. For example, as described in Chapter 2 , parents seemed to be influenced by their children's need for independence (e.g. taking initiative in or being curious about sun protection measures), as well as a preferred parenting style (e.g. being in control over their children's health). For example, parents performed less indirect behaviours when their child was eager to perform sun safety measures themselves, and more frequently performed supportive behaviours when they preferred to be in control over their child's health. As has been discussed in other health behaviour practices such as dietary intake $(85,392,393)$, children's characteristics, besides parental preferences, can influence parenting strategies or styles. Investigating the role of parent's and children's characteristics in sun protection behaviours might be interesting to ultimately develop a tailored approach addressing characteristics of the individual child.

\section{Children's own sun protection behaviours}

In addition to parental sun protection behaviours, this thesis also explored children's own sun protection behaviours, by using parent-proxy reports (consider Chapter 4). Although children in our sample (4-12-year olds) still seemed largely dependent on their parents, our study has shown that children are performing sun safety measures themselves, and especially sunscreen and clothing.

Firstly, when investigating the extent to which children were performing sun protection behaviours themselves, our analyses revealed that children were most often engaging in sunscreen use, in planned 
situations, followed by clothing behaviour in both sun exposure situations. Children's own shade-seeking behaviours were least often reported. Although children's own behaviours have not been thoroughly investigated so far, comparable results have been reported before in which children mostly applied sunscreen $(48,50,82,306)$.

Secondly, although sun safety behaviours were mainly demonstrated among the oldest children (10 to 12 years old) in our sample, sun protection behaviours were also sometimes reported among the youngest children ( 4 to 6 years old). Among these youngest children, clothing behaviour was reported most often. Our findings are contrary to earlier research, in which sun protection behaviours of children decline when they are getting older $(77,247)$. Our results could be partly explained by our findings in Chapter 4 , in which parental supportive behaviours remained high across children's ages. The older children in our sample might receive great encouragement from their parents, and are stimulated to perform their own sun protection behaviours.

Thirdly, we explored whether a behavioural shift takes place, in which children performed more sun protection behaviours themselves than their parents did for them. Our results revealed that children aged 11 years old appeared to wear protective clothing themselves to a greater extent than parents used protective clothing for them in planned sun exposure situations. For all other behaviours, behavioural shifts were predicted by extrapolation, around the age of 14. It is positive that our study indicated that older children execute more sun safety measures since the majority of studies including parental reports show that children perform less sun protection behaviours as they are getting older $(51,77,80,170)$. Interventions focusing on older children, emphasizing their own sun protection behaviours, might therefore increase their levels of sun safety.

Fourthly, when further exploring children's characteristics with regard to their own sun protection behaviours, some interesting findings emerged. First of all, girls seemed to perform sun protection behaviours more often than boys, as outlined in Chapter 4 . This finding echoes results from previous research $(247,257,259$, 394). Moreover, previous research indicates that boys are prone to spend more time outdoors $(80,395)$, and are more vigilant for sunburn $(76,260,383)$. Although the latter was not reported by parents in our studies, possible existence of these patterns could place boys at greater risk of unprotected sun exposure than girls in this age ( 4 to 12 years old).

\section{I.3. Wisdom begins with wonder: Understanding sun protection behaviours}

We aimed to investigate the establishment of parental sun protection behaviours from a socio-cognitive, as well as environmental perspective. Results from our longitudinal study as described in Chapter 3 , identified; 1) moderate to high overall explained variances of parental sun protection behaviours by socio-cognitive determinants; 2) an overall importance of specific motivational - especially attitude and self-efficacy determinants in the explanation of parent-for-child sun protection behaviours; 3 ) the relevance of postmotivational (action planning) and pre-motivational (knowledge and risk-perceptions) determinants in explaining parental behaviours; 4) a positive attitude among parents concerning a tanned skin; and 5) the importance of many characteristics in the physical environment, either facilitating or hindering parental sun protection behaviours.

\subsubsection{The socio-cognitive perspective}

\section{Explaining sun protection intentions and behaviours}

When investigating the predictive value of a wide range of socio-cognitive determinants on parental sun protection behaviours, two interesting findings emerged. 
Firstly, the overall explained variance of parental sun protection behaviours by socio-cognitive determinants ranged between 19 and $41 \%$ in planned, compared to 28 and $43 \%$ in incidental sun exposure situations. Furthermore, shade-seeking (42\%) and clothing (36\%) behaviours showed higher explained variances than sunscreen use (31\%) and indirect (24\%) behaviours. Earlier research, which lacked differentiation of various sun protection behaviours and sun exposure situations, and primarily focused on younger children, has reported comparable findings. For example, in studies investigating the predictive value of socio-cognitive determinants derived from the health belief model (HBM) and the Theory of Planned Behavior (TPB), the explained variance of parental sun protection behaviours for younger children (aged between 2 and 6) ranged from 36 to $55 \%(118,119,130)$. Since only few former studies reported on explaining multiple parental sun protection behaviours for children, comparisons with our findings are challenging. Yet, our explained variances seem worthy of comparison since a meta-analysis revealed that socio-cognitive determinants on average explain $27 \%$ of a wide variety of other health behaviours (396).

Secondly, we identified lower explained variances for sun protection behaviours than for intentions. These differences might display the well-known intention-behaviour gap in which intentions are not fully translated into behaviour (397). Our findings therefore suggest the potential importance of post-motivational determinants, such as action and coping planning, in encouraging motivated parents to bridge the gap to executing sun protection, as has been mentioned before for adult sun protection behaviours (127) and for sunscreen use among parents (133) and students (398). Moreover, this intention-behaviour gap could further be explained by habitual factors as well as environmental factors.

\section{Motivational determinants}

As described in our longitudinal determinant study in Chapter 3 , the more proximal determinants of behaviour change demonstrated highest relevancy in explaining parental sun protection intentions and behaviours. These motivational determinants (especially self-efficacy and attitude) showed high associations with parental sun protection behaviours, as well as notable room for improvement. Most importantly, since the majority of existing parent-focused sun safety interventions generally focus on premotivational determinants $(82,159)$, our findings illustrate the need for targeting the following motivational determinants.

Firstly, self-efficacy, and especially perceiving sun protection behaviours as difficult, demonstrated the lowest mean scores and thus notable room for improvement. As echoed in previous work, when parents experience sun protection performance as a hassle, the likelihood of performing protection behaviours decreases as low self-efficacy is a barrier for enactment $(119,399)$. Since previous research stated that besides intention, self-efficacy constructs directly influenced parental sunscreen use (133), these results advocate for increasing parental feelings of ability by decreasing perceived barriers. However, we need to gain specific insight into difficulties hindering parents to perform sun protection measures in order to address these barriers in interventions and to help parents to overcome them.

Secondly, attitude in which both importance and pleasantness of performing sun protection behaviours was assessed, revealed high relevancy as well. Compared to other behaviour-specific determinants, attitude showed lowest room for improvement due to high sample mean scores, but high associations with the outcome intentions and behaviours. Previous research has shown likewise results $(80,163)$ with a favourable attitude of sun protection among parents being an important predictor for their sun protection behaviours $(75,131)$. Since parental attitudes concerning sun protection have a strong influence on children's own sun protection attitudes $(51,79,263)$, it is encouraging that parents seem to have positive attitudes concerning various sun protection behaviours. It should be noted that we assessed attitude by means of two beliefs. Since these beliefs were important in predicting parental sun protection behaviours, the relevance of other attitudinal factors, e.g. consciousness raising, pros and cons and self-re-evaluation (400), can be investigated in order to provide specific recommendations for interventions.

\section{Post-motivational and pre-motivational determinants}

Besides motivational determinants, other relevant determinants were identified. 
Firstly, findings in this thesis suggested an important role of the post-motivational determinant action planning in predicting parental sun protection intentions and behaviours. The association of action planning - conceptualized as preparatory planning - was high across all behaviours, implying an important role of formulating preparatory plans in the execution of protection behaviours. The influential role of action planning has been demonstrated before in research investigating the onset of parental sunscreen behaviour $(133,239)$ and multiple parental sun protection behaviours $(131)$. Since formulation of preparatory plans can increase the likelihood of intentions translating into behaviours (401), further investigation of this determinant is worthwhile. Other post-intentional concepts such as goal-setting, capacity of selfregulation or habit $(128,131,244)$ also seem worthy of further investigation in the context of sun protection behaviour. Particularly, the importance of coping planning in enacting parent-for-child sun protection intentions into behaviours seems interesting, since parents perceived many difficulties in performing sun protection behaviours, as described in Chapter 2. Anticipating on such difficulties and planning efficient coping responses may further increase the likelihood of enacting the desired sun protection behaviours $(402,403)$.

Secondly, pre-motivational determinants were also identified as important determinants explaining parental sun protection behaviours. Generally, these pre-motivational factors, such as knowledge and riskperceptions, are regarded as prerequisites to behaviour change $(162,404)$. However, only few studies have examined their influence on parental sun protection behaviours $(81,95)$. As described in Chapter 3 , the general level of skin cancer related knowledge was fair among parents and was moderately associated with sun protection behaviours. However, as outlined in Chapter 2, misconceptions about adequate sun safety and the necessity of sun protection measures were also detected. Even among seemingly highly aware parents, we found deficits regarding correct sunscreen application, interpretation of the UV-index and the need for conjunct use of sun protection measures. Moreover, misconceptions about adequate sun safety are likely to be translated into insufficient sun protection behaviours and subsequent unawareness about the inadequacy of these behaviours. Our findings are comparable to previous work, where insufficient knowledge about the need for sun protection strategies among parents has been described $(53,114,161)$. Studies have shown that sun protection knowledge is not sufficient for changing sun protection behaviours among adolescents and adults $(405,406)$ and among parents $(154,161)$. Besides knowledge, risk susceptibility in incidental sun exposure situations demonstrated room for improvement and moderate associations with sun protection behaviours, as described in Chapter 3. We measured both cognitive (estimations of risk) and affective (feelings of risk) susceptibility perceptions (407). Parents particularly perceived low cognitive and affective susceptibility perceptions in incidental sun-exposing situations. Together, the results implied that specified knowledge, that is, tailored information concerning adequate sun protection strategies and when and where to apply them, and increasing susceptibility perceptions in especially incidental sun exposure situations, is warranted.

\section{Tanned skin}

Besides behaviour-specific cognitive determinants, we found several interesting results with regard to parents' attitude regarding tanned skin.

Firstly, the majority of parents reported a positive attitude towards having a tanned skin for both themselves and for their children, a finding described in Chapter 2. Parents perceived tanned skin as more beautiful, healthy and more attractive and were often complimented by peers on their - or their children's - tanned skin. Deeply tanned skin was however regarded as tacky or ugly. Comparable results have been found among general populations as well as parents specifically $(51,79,101,161)$.

Secondly, a negative association between the parental attitude concerning children's tanned skin and sun protection intentions and behaviours, was demonstrated (see Chapters 2 and 3 ). This worrisome finding is also reflected in previous studies, irrespective of high awareness and knowledge concerning skin cancer risk $(51,52)$ and even among parents of very young children $(161,408)$.

Thirdly, parents reported a common social norm in which tanned skin is perceived as positive. As outlined in Chapter 2, parents were influenced by compliments from their peers about their own and children's tanned 
skin. This finding implies a prevailing cultural norm that idealizes tanned skin in the Netherlands, which has been earlier described as Western cultural ideal (409). Altogether, addressing the positive norm regarded tanned skin needs addressing, most importantly since children's sunburn risk can increase when parents are in favour of a tanned skin (52).

\subsubsection{The environmental perspective}

Many characteristics in the physical environment, either facilitating or hindering parental sun protection behaviours, were revealed, as described in Chapter 2. Moreover, in Chapter 6, we outlined current evidence of environmental cues improving sun protection behaviours of children.

\section{Health promoting environment}

While the importance of cognitive determinants on parental sun protection intentions and behaviours was demonstrated in this thesis, direct effects of environmental determinants and characteristics on parental and children's sun protection behaviours was also notable, as illustrated in Chapters 2 and 6 .

As described before, parents interviewed for this thesis perceived many barriers in outdoor settings, especially in situations involving water-related activities. The unavailability of shade and the notion that aquatic activities make adequate sunscreen application more difficult prevented parents most often from protecting their child sufficiently. Ultimately, this often resulted in longer durations of children being unprotected and a higher risk of sunburn. Since these settings are high-risk situations, in which parents in our studies demonstrated high levels of sun exposure, efforts to reduce such barriers are regarded highly beneficial.

The potentially important role of health-promoting environments in the execution of sun protective behaviours has recently been observed among adolescents and adults at public venues in Australia (339). However, a systematic review investigating the effectiveness of health-promoting interventions at recreational venues specifically, gathered inconsistent and limited evidence (143) and reported no evident improvements since a previous and similar review conducted 12 years earlier (82). In Chapter 6, we reviewed the effectiveness of environmental sun safety interventions focusing on children and adolescents. Although the evidence was limited, we found that sun protection cues in the environment can evoke sun protection behaviours. Availability of shaded areas showed most promising results in terms of increased shade-seeking behaviour, followed by provision of protective clothing and freely available sunscreen. The importance of availability of shaded areas was also reflected in our qualitative study; when parents were asked what they perceived most helpful in enhancing sun protection behaviours during interviews, the majority mentioned the possibility to seek shade. Together with reminding cues, e.g. warning signs displaying current UV-index, and free provision of sunscreen, parents would feel more encouraged in enacting sun protection measures.

\section{Part 2. Strengths and limitations}

\section{I. Methodological strengths}

Overview studies show that the majority of sun safety interventions are not directed at the parent-child interaction $(82,158)$, even though parents are regarded imperative in order to enhance sun safety in childhood (210). This thesis provides insight in sun safety of Dutch children from a parental perspective, while including children's sun protection behaviours as well. Furthermore, we were able to fully cover the primary school age (4 to 12 years) of children in our studies, thereby displaying an important phase in childhood for 
commencing sun safety efforts (77). The samples that were used to perform quantitative data analyses were all part of a nation-wide longitudinal study. As a result, all of these studies had relatively large sample sizes, which generated data of 500 to 1299 parent-child pairs, thereby increasing statistical power for statistical analyses (410). Moreover, by making use of a sample representative of the Dutch general population based on education level and income, both sampling and coverage errors were limited (411).

The studies outlined in this thesis were characterized by the extensive assessment of parental sociocognitive determinants as well as comprehensive sun protection behaviours, while disentangling these parental constructs for different sun-exposing situations. Since only few former reported studies regarding children's skin cancer prevention included more than two parental constructs (412), the assessment of an elaborate set of determinants derived from the I-Change Model (162), enabled the identification of essential concepts (both comprehensive generic and behaviour-specific), reaching beyond pre-existing literature.

The strength of the presented research is further increased by the use of mixed study designs. The use of both qualitative and quantitative methods, as well as the execution of a systematic literature review has yielded in-depth exploration of Dutch children's sunburn, parents' sun protection behaviours, sociocognitive determinants influencing these behaviours, and potentially effective interventions.

\subsection{Methodological limitations}

Two main methodological shortcomings should be considered when interpreting the findings from our studies.

Firstly, in our longitudinal survey study, described in Chapters 3, 4 and 5, answers were based on parental self-reports, thereby increasing the risk of biases. Since most questions were assessed in retrospect, a recall bias may have been at play (300). While stressing the recall period in the survey questions, this bias should be acknowledged when interpreting the results presented in this thesis. We strived for the most optimal sunburn measure by asking the frequency of sunburn during a certain timeframe, which is comparable to most studies assessing sunburn in children (143). Moreover, although self-reported parental sunscreen use (248), and self-reported sun exposure has shown to be valid among general populations and parents (272, 304), this method is unable to integrate vital information such as cloudiness, altitude or shade coverage, which restricts specific risk-profiling (93). We aimed to decrease a self-report bias by basing our measures on a validated sun exposure and sun protection questionnaire (413). Furthermore, although the majority of children-focused sun protection measures rely on parent-proxies $(414,415)$, subjective assessments can increase the risk of parents providing socially desirable answers (416). For example, although the majority of studies rely on self-reports, its validation is poor, leading to underestimations of sunburn occurrence among adults (417) and in parental reports of children's sunburn (198). Particularly since questions concerning parenting-type behaviours were asked, over reporting of their protective behaviours or underreporting sunburn may have occurred. We attempted to reduce the likelihood of such bias by stressing the anonymity of the questionnaires and further minimized response biases by performing online and telephonic instead of in-person measures.

Secondly, the analyses described in Chapters 4 and 5 were based on cross-sectional data, which limits the assumption of causal relationships. The ultimate aim of this thesis was to increase knowledge of primary skin cancer prevention in the Netherlands. Since cross-sectional designs allow for explorations of prevalence of various health-related outcomes, exposure to risk factors and related participant's characteristics (418), the data of these Chapters were in line with our preliminary goals. Moreover, many results from the crosssectional data showed correspondence with findings from both the longitudinal and the qualitative data. However, it would be worthwhile to replicate the findings concerning behavioural patterns of parents and children longitudinally, as described in Chapters 4 and 5. 


\section{Part 3. Recommendations}

\section{I. Recommendations for future research}

\section{Objective measurement of behavioural outcomes}

Although objective measurement of individually received UVR has been advocated for since the beginning of the century, currently objective UV dosimetry among children, specifically in the Netherlands, is scarce (419). Since skin cancer has a latency of at least 5-20 years after UVR-induced damage (420), we need to gain insight in the objective amounts of children's sun exposure and sunburn. In order to ultimately assess effectiveness of interventions in terms of behaviour change, objectifying these behaviours is recommended. Combining self-reported assessments with objective measures, e.g. in order to gain information on exposure patterns has been advised in earlier work $(413,414)$. Objectifying exposure can be accomplished by applying a relatively easy and inexpensive method, e.g. Polysulfone (PS) film (304, 305), which provides data on cumulative doses of UVR. Personal dosimetry devices, working electronically, are increasingly used in the field of objective measurements (421), and tested among children specifically (169, 422), since they permit assessment of exposure magnitude and activities. Besides gaining insight in children's sun exposure in high-risk situations, usage of personal UV-dosimetry can open doors to real-time and tailored risk communication, which has gained increasing attention among adolescent and adult groups (423-425).

In relation to this, since a large variability in reported sunburn was found across studies in this thesis and abroad, the objective assessment of sunburn among children would yield valuable information. By objective sunburn measures, biases due to social desirability or misconceptions about sunburn can be prevented. A recent study evaluated the use of an objective device assessing sunburn - through levels of melanin (tanning) and erythema (redness) of the skin (426) - among adolescents and demonstrated high feasibility (302). In order to increase our current knowledge on high-risk situations with regard to sunburn, insight in objective sun exposure and sunburn seems warranted. Additionally, this insight can improve future intervention content based on these high-risk situations and assist parents in performing sun protection behaviours by providing tailored coping plans.

\section{Exploring the gap}

We identified an intention-behaviour gap and indicated the importance of action planning in explaining parental sun protection behaviours. Self-efficacy - especially feelings of difficulty - with regard to performing sun protection behaviours, appeared relevant as well. These findings ask for further investigation of other factors possibly related to behaviour change.

Firstly, examining the role of coping planning by gaining insight in specific difficult situations and knowing which barriers prevent parents from adequately performing sun protection behaviours, provides detailed directions for tailored interventions $(102,189)$. Testing the relevance of additional beliefs could be strived for in order to detect 1) difficult situations and/or settings, and 2) whether parents have specific plans to cope with these situations. Moreover, by utilizing the personal UV-dosimetry devices - ideally integrated with GPS, risk situations and characteristics of high-risk environments can be objectively captured, providing more salient information. Acquiring this information can provide directions for formulating coping plans and implementation intentions in interventions $(402,427)$.

Secondly, investigating the possible roles of habitual and past behaviour in unravelling parental sun protection behaviours is worthy of consideration since the results described in this thesis indicated a possible pattern of inadequate behaviour, possibly reflected in sunburn. As past behaviour is regarded as a 
proxy for habits as demonstrated for other health behaviours $(428,429)$, both concepts are highly relevant to explore further. Additionally, a recent meta-analysis about several parent-for-child health behaviours, illustrated that past behaviour predicts current behaviours and the role of habit was suggested to mediate the effects of this relationship (430). It would be highly relevant to know if and which behavioural patterns and habits exist, e.g. habitual sun-seeking instead of shade-seeking behaviour, or wearing less covering clothes instead of long-sleeved T-shirts and trousers.

\section{The physical context}

In our systematic review we gathered preliminary evidence demonstrating that environmental cues can improve children's and adolescents' sun safety levels. Since the amount of studies specifically focusing on children and their parents was limited, more research is recommended in order to answer the question which characteristics in the environment influence parental and children's behaviours (431). More importantly, since behaviour is largely dependent of external factors such as sociocultural influences and characteristics in one's physical, social or political environment (112), further investigation of the potential influence of such factors in a Dutch context is warranted. Firstly, behavioural patterns in outdoor settings, e.g. schoolyards, playgrounds, public parks, and swimming pools, can be detected by using objective measurement devices or observational study designs. Secondly, by manipulating environments through implementing nudges or behavioural cues, e.g. shade sails, warning signs or freely available sunscreen, effectiveness on sun protection behaviours can be tested, using randomized controlled designs. Besides our findings described in this thesis, recent international research shows encouraging effects of (purposely built) shade on shade-seeking behaviour among general populations (144, 339). Knowing the amount of sun exposure that children receive in which type of environment, as well as investigating the effects of environmental cues on sun protection behaviours in different settings, enables interventions to be tailored to high-exposure environments.

\subsection{Implications for practice:
the micro-level}

By contributing to existing literature regarding children's sun safety, we aimed to provide directions for future parent-focused skin cancer prevention interventions. Composing interventions directed at the family-setting is strongly advised since children are still largely dependent on parent's protective behaviours and our results revealed room for improvement in parental sun protection cognitions and behaviours. Furthermore, implications for targeting and tailoring interventions regarding several themes of interest emerged.

\subsubsection{Developing educational content}

When intervention content and communication is tailored to one's motivation, risk factors and personal features, people are more prone to be persuaded and change their behaviour, as demonstrated in a large body of health behaviour research (121). Given the complexity of performing adequate sun protection behaviours, tailored messaging is regarded crucial as well $(122,123)$. This thesis identified various relevant foci and implications for tailored messaging in future sun safety interventions.

\section{Behaviours: shade and clothing, besides adequate sunscreen}

Firstly, since the parents in our study samples reported clothing and seeking shade less often than using sunscreen, future sun safety interventions should emphasize the importance of clothing and shade. Besides a strong focus on shade and clothing measures and the simultaneous performance of these behaviours, adequate sunscreen application needs further attention as well. As demonstrated in this thesis and in line 
with previous documented research, parents perceive sunscreen use as highly difficult. In order to facilitate parental sunscreen use, parents for example can be educated on how to correctly apply sunscreen, when to apply and reapply it, on which body parts to use it, and how to instruct children to apply sunscreen themselves (432).

Secondly, our determinant analyses identified greater explained variances for seeking shade and clothing behaviours than for sunscreen use. Educational efforts that target socio-cognitive determinants of shade-seeking and clothing behaviours might therefore be effective in enhancing these behaviours among parents. Although the possibility of seeking shade and sun avoidance is closely related to the physical environment, parents can be encouraged to facilitate sun protection by bringing their own shade equipment or scheduling their activities when UV-index is low. To improve clothing behaviours, parents can be stimulated to anticipate on sun exposure by providing UV-protective clothing or long-sleeved and long trousers, sunglasses, and hats $(82,138,332)$. By informing parents on how and in which situations to best use clothing and to provide them with these garments, children's sun safety can be improved.

\section{Sun exposure situations: planned versus incidental}

This thesis provided insight in different sun exposure situations, a subject that deserves further attention.

Firstly, since our results indicated low feelings of risk susceptibility in incidental sun exposure situations, together with a possible underestimation of sun exposure risk in such situations, parents should be made more aware about the importance of sun safety strategies in these everyday settings. As to our knowledge, no evidence of the effectiveness of interventions targeting these settings exist. Earlier work regarding parental sunscreen use found that risk-perceptions influence motivational determinants such as attitude and self-efficacy (132), and observed better sun protection behaviours on the beach among parents with high risk knowledge (289). Since risk-perceptions influence motivational determinants $(132,404)$, parents should obtain accurate knowledge about necessary sun protection measures and be convinced about the risks of incidental sun exposure in order to decrease children's sunburn risk.

Secondly, we identified high-risk situations, that is, when parents and children are engaging in waterrelated activities. Parents in our studies perceived adequate sun protection in these situations as difficult, and children seemed more prone to experience sunburn. For activities involving water specifically, parents could be instructed to take additional measures to overcome barriers (150), such as bringing a parasol, putting on long-sleeved clothing $(289,332)$ and reapply sunscreen more often.

\section{Addressing determinants}

In conclusion, while most prior international sun safety interventions generally focus on pre-motivational determinants $(77,82)$, we advocate for a stronger focus on motivational and post-motivational determinants while not overlooking the need for addressing pre-motivational determinants.

\section{- Motivational determinants}

Since our studies indicated an overarching importance of specific motivational and post-motivational determinants, we provide recommendations for addressing these concepts in future interventions. Firstly, parental feelings of ability and difficulty to perform sun protection behaviours should be addressed. Increasing mastery experiences (433), by successfully accomplishing different sun protection behaviours may enhance parental self-efficacy. Previous interventions focusing on increasing self-efficacy, e.g. by using parental role models, have shown some positive results on parental sun protection behaviours $(222,432$, 434). When insight in high-risk situations is optimized and specific difficulties are further revealed, a more tailored approach in order to increase parental self-efficacy can be applied (197). Secondly, a modification of the perception that tanned skin is positive is recommended. Since our results implied that important others were reinforcing tanned skin as well, this might indicate an intrusive cultural norm encouraging the pursuing of a tanned skin, which is displayed in Western societies $(409,435,436)$. Reshaping the image that tanned skin is aesthetically ideal, should begin as early in life as possible since parents have a leading role in influencing children's attitudes towards sun protection (79). More importantly, since physical appearance becomes more important whereas sun protection attitudes decline around adolescence $(79,98,437)$, 
childhood is an important stage to address the positive attitude regarding a tanned skin. Furthermore, since appearance-related norms are often a result of interactions between macro level (cultural values and norms) and micro level (peers or family) influences (438), the implementation of public health campaigns to address this beauty ideal on a societal level seems warranted (439). Although the effects of such initiatives addressing tanned skin particularly are unknown, positive results of public campaigns and interventions on attitudes towards tanning behaviour have been reported $(440,441)$. Finally, it might be worthwhile to address the social norm regarding tanned skin by integrating appearance-focused elements in interventions (442).

- Post-motivational determinants

To increase the likelihood that motivated parents will enact their intentions into performance of sun protection behaviours, parents could be encouraged to formulate implementation intentions (239). As was stated before, insight in difficult situations hindering parents to adequately perform sun protection strategies is needed in order to develop tailored approaches assisting parents in coping with these difficulties. Where action planning refers to when, how and where parents will perform sun protection strategies, coping planning anticipates on barriers and provides strategies to overcome them (402). By linking internal and external cues to specific predetermined actions, the goal-directed actions are performed more automatically. This increases performance of health behaviour and is thought to reduce the influence of existing unhealthy cue-response linkages, or habitual behaviour (443). Although the evidence for the influence of coping planning on sun protection enactment is limited (444), the positive effects of formulating coping plans - particularly in combination with implementation intentions - for other health behaviours $(403,445,446)$, could possibly be translated to sun protection behaviours as well.

\subsubsection{Targeting of interventions}

Our studies indicated specific groups of children at-risk in terms of sun protection and sunburn. With regard to targeting of future interventions, two findings of our current studies are noteworthy to mention.

Firstly, we recommend that future interventions focus on older children, since research presented in this thesis indicated that older-aged children (especially between 10 and 12 years old) are less protected with sun protection and more prone to experience sunburn. Moreover, we found that older children are increasingly performing sun protection behaviours themselves. When entering the adolescent phase, appearance and peer influence become increasingly important and may negate earlier established sun protection attitudes and behaviours (71). Thus, targeting on older aged children - especially when commencing adolescence -, is warranted $(79,159,259)$. However, the whole primary school age should be taken into account in intervention development since establishment of sun safety attitudes and behaviours in these ages increases the likelihood of maintaining these behaviours in later life $(77,79)$.

Secondly, findings described in this thesis (see Chapter 4) suggested that boys performed less sun protection behaviours than girls, but were not receiving higher doses of (unprotected) sun exposure and were not at greater risk of sunburn. Replication studies are important to investigate the perpetuation of these results, but based on our current findings, tailored messages for boys, e.g. focusing on relevant contexts such as a football field, seem warranted. Girls might be at greater risk for receiving high amounts of (unprotected) sun exposure when growing older, by becoming more appearance-focused and engaging in tanning behaviours when reaching adolescence $(79,257,437)$. Therefore, developing messages for girls especially when reaching adolescence - in interventions is imperative as well. 


\subsection{Implications for practice: the meso- and macro-level}

A multi-system approach towards intervention development, addressing various levels of influence, is further recommended since the parent-child interaction, occurring in the micro setting, is only one of the levels influencing children's behaviour. Parenting does not only take place in this micro-level, but is influenced by and a result of interactions between multiple systems, e.g. the meso-level (school, communities) and the macro-level (public policy, cultural norms) (447-449). Within these systems, interaction between various types of environments can shape children's behaviours $(112,450)$. A recent review found limited progress in the development of multi-level sun safety interventions and indicated notable room for improvement (451). Altogether, a comprehensive multi-level strategy to combat skin cancer in the Netherlands is specifically recommended.

\section{Meso level}

The primary school can be a promising setting to implement sun safety programs (82), since both children and their parents can be easily reached. A study conducted among parents in the Netherlands, suggested a lack of sun safety policy at primary schools, which was reflected by over $20 \%$ of children having experienced sunburn at least once at school and over 30\% during school activities (54). Moreover, it was observed that over $20 \%$ of children had experienced sunburn at least once at school. At the primary school, both educational interventions, physical interventions (e.g. shaded playgrounds) as well as policy (e.g. scheduling breaks outside UV-peak hours, mandatory hat use) can be implemented to enhance children's sun safety $(139,223)$. At schools, but also at outdoor areas on a community level, environments could be altered in a way that parental and children's sun protection behaviours are facilitated (6). One opportunity for sun safety programs in Dutch primary schools can be found within the Healthy School approach, a health promoting concept that builds on schools' needs and current policy and is advocated by the Dutch National Institute for Public Health and the Environment (452). Programs aimed at improving children's health at primary schools in the Netherlands have shown to be effective in improving dietary intake and physical activity (453). Therefore, optimizing the incorporation and integration of sun safety initiatives in the healthy school program and testing their effects is required.

As the findings reported in this thesis, the physical environment might be an encouraging setting to intervene. Since recreational and tourism settings were previously found to be effective settings for implementing sun safety interventions (82), shaping children's physical environment by implementing sun protection cues (e.g. built shade, sunscreen provision) seems worthwhile. Public parks, playgrounds and outdoor recreation or exercise settings could benefit from these alterations (451).

\section{Macro level}

In order to achieve sustained change on a population level, effective sun safety interventions must be widely disseminated, as was recently advocated for by scientists and experts from several fields (e.g. dermatology, behavioural medicine, public health) (123). Policy or regulations on a public level can reach a broad audience while requiring minimal effort for individuals to make healthier choices $(6,112,145)$. Macro level interventions can consist of indoor tanning legislation (e.g. adding warning signs on tanning beds, increasing the excise duty), providing real-time information on the UV-index, regulation of sunscreen products (securing the broad-spectrum protection), and broad distribution of communication by using mass or social media communication for campaigns $(6,286)$. In addition to this, legislation for environmental design in which shade becomes a focus within urban planning, by e.g. creating incentives for providing shade at outdoor venues, might be promising as well $(144,167)$. A combination of approaches is recommended, since evidence for effectiveness of mass media strategies on sun protection behaviours is insufficient (223). 
The most salient example of a macro level approach in order to combat skin cancer can be seen in the country identified as the world's 'skin cancer capital' Australia (454). Their macro-level SunSmart program consists of, among others, media campaigns to increase public awareness and promote protective behaviours, school-based educational interventions, provision of incentives for organizations to improve sun safety policy and utilizing role models for children and adolescents at outdoor events (137). Three decades of the program has demonstrated population increases in sun protection behaviours, and conceivably contributions to decreased melanoma rates (138). In addition, almost 30 years of observation research concurrent to health-promoting SunSmart activities has demonstrated increased clothing coverage among adolescents and adults during peak UV-hours at outdoor venues (455). Another promising example is the Danish Sun Safety Campaign, a multi-component program in Denmark including among others, (social) media campaigns, an UV-index application, press coverage and political debate, implemented in various settings such as sports clubs, kindergartens, and schools. The positive results of this strategy were reflected in notable decreased population sunburn rates and predictions of lower skin cancer rates (456). Moreover, this program has shown to be cost-effective and predicted decreased skin cancer care costs for the Danish government (457).

As these results set a great example, the political opportunities for policy change in the Netherlands cannot be compared one-on-one with other countries. Translating macro-level programs to a Dutch context seems worthwhile, as is further discussed in the Impact paragraph in this thesis. Recent developments have opened a window of opportunities to advance nation-wide strategies regarding skin cancer prevention. It is therefore time to explore the options and seize these opportunities to counter the problem of skin cancer in the Netherlands. 


\section{Impact Paragraph}




\section{Skin cancer in the Netherlands}

The Netherlands is among the five countries globally showing highest skin cancer rates. The time to act is now; estimations show that in the next decennia, skin cancer rates will be two to even five times higher (11). Unprotected sun exposure and sunburn are the most important risk factors for skin cancer, making it a highly preventable disease. Sun safety - being safe in the sun - for children is particularly important since sunburns during childhood profoundly increase skin cancer risk. As insight in children's sunburn, sun exposure and sun protection is very limited, our efforts to unravel children's sun safety in the Netherlands are imperative and timely.

\section{Understanding children's sunburn, sun exposure and sun protection}

As parents bear the main responsibility for their children's sun protection and are the most important caregivers and educators, we aimed to unravel children's sun safety from a parental perspective. The purposes of our research were threefold: 1) increasing knowledge about sun exposure and sunburn among children (aged 4 to 12 years old); 2) gaining insight in parents' and children's own sun protection behaviours, and; 3 ) understanding parental sun protection behaviours from an individual and environmental perspective. In brief, our most interesting findings indicated that;

1) More than 4 in every 10 children experienced one or more sunburns every year, with older children showing higher sunburn risk than the younger. Parent's reports indicated that children were often exposed to the sun's damaging UV-radiation in both high-risk situations (such as going to the beach or swimming pool) and while engaging in everyday activities (such as cycling or playing outside). Parents seemed less aware of sun exposure risks in these latter situations. Further, limiting sun exposure in high-risk situations seemed to decrease children's risk of sunburn.

2) Parents engaged in sun protection behaviours frequently and applied sunscreen most often, followed by putting on clothing and seeking shade. Parents also frequently supported their children in performing their own sun protection behaviours. Sun protection was more often performed in earlier mentioned high-risk, than in everyday situations. Among children themselves, older children and girls performed sun protection strategies most frequently.

3) From an individual-based perspective: a) parents' attitudes about sun protection behaviours, confidence about performing sun protection and preparing for sun exposure by making plans (e.g. making sure a bag with sunscreen is always readily available) were most important in predicting their behaviours; and b) parents held a positive attitude towards a tanned skin - indicating that they believed it looks more beautiful and healthy on their child, which may interfere with their sun protection behaviours. From an environmental perspective, we found that: a) many aspects of the physical environment either hinder (e.g. water-related activities burdening efficient sunscreen application) or promote (e.g. facilitating sunscreen or shade) parental sun protection behaviours; and b) some environmental 'cues' encouraging sun protection, such as provision of hats or shaded areas at schools or at playgrounds, are effective in enhancing children's sun safety. 


\section{The relevance of our findings}

As high sunburn rates were found despite parents' fair reported sun protection behaviours, we conclude that sun safety among children in the Netherlands is in need for improvement. We recommend that besides targeting on groups (i.e. older children and boys), and developing tailored educational content (e.g. on attitudes, self-efficacy and action planning), the physical environment should deserve a role in future interventions.

We expect our results to have a potential impact on 1) development of public health interventions focused on children and parents, 2) the current and future scientific field of primary skin cancer prevention, and 3) the development of a national skin cancer prevention strategy in the Netherlands.

\section{Practical relevance}

We indicate that sun safety interventions for children and parents are warranted. Program planners can take into account the many concepts we identified related to children's sun safety when planning health education and health promotion programs. We provided recommendations for: a) educational content; and b) alterations in the physical environment.

Firstly, parents need education to increase their awareness about sunburn, sun exposure risk and effective sun protection strategies and guidance in how to perform them adequately. Furthermore, in preparation for the shift in behavioural responsibility, parental role modelling and support and children's mastery of own sun protection behaviours need focus in future interventions.

Secondly, characteristics in the environment, such as establishing shaded areas or providing sunscreen or sun protective clothing, are worthy of consideration for future interventions. Besides intervening in the family setting, our practical recommendations can also be applied to other settings where children are sun-exposed, such as at school, sports clubs or outdoor recreation. Health promotion professionals and policy makers at Municipal Public Health Services, communities, primary schools, after-school care, in urban design, and sports clubs can benefit from our results by taking the above recommendations into account when designing and implementing (multi-component) interventions.

\section{Scientific relevance}

We contributed to a scientific field that was so far limited in the Netherlands. We aimed to make our scientific output widely available, by posting our findings on the Open Science Framework (OSF) and submitting our work in Open Access Journals. Besides scientific publications, our main findings were also presented at both national (public health and health psychology focused) and international (skin cancer prevention focused) conferences. Furthermore, we organized a symposium consisting of international researchers involved in (primary) skin cancer prevention from the Netherlands, Australia, the United States and Denmark that will take place in the Autumn of 2021.

Scientists can benefit from our research when developing evidence-based sun safety interventions for parents and children and when ultimately systematically evaluating these interventions. While the composition of evidence-based skin cancer prevention programs is highly warranted, they are evidently lacking in the Netherlands up until now. It should be strived for that skin cancer prevention interventions are composed and officially recognised by the Centre for Healthy Living, thereby reaching broader audiences and enabling systematic evaluation. Further, since we identified gaps in the current scientific literature, we provided recommendations for further research, namely: a) objectively assessing children's sunburn and sun exposure; and b) investigating parental and children's sun protection behaviours in manipulated settings. 
Firstly, our studies provided indications of children's sunburn and sun exposure, but objective information is currently missing. Objectively monitoring children's sunburn incidence and sun exposure patterns is likely to reveal more in-depth information regarding at-risk groups and at-risk situations or settings. This information will further optimize the content and targeting of interventions.

Secondly, we indicated that adaptations in the physical environment can encourage sun safety strategies. Since the effects of environmental cues on sun protection behaviours were not tested in a Dutch setting, our findings suggest developing, testing and, when proven effective, disseminating and testing such interventions (e.g. provision of shade and free sunscreen) in the Netherlands. This can provide information on sun protection preferences and shortcomings, which enables us to provide specific directions for interventions. Thus, studies comparing different types of environments, observing behavioural patterns and investigating the effects of implemented cues could be worthwhile. These future studies enable interventions to tailor on high-risk settings or at-risk groups, thereby potentially enhancing effectiveness of these interventions.

\section{Societal relevance}

Formulating a skin cancer prevention strategy that is adhering to societal needs, norms and considers the current political climate, is warranted. As the Netherlands faces many other public health challenges, the findings in this thesis can function as an entry point to put primary skin cancer prevention (more) on the political agenda. Our scientific efforts have potential impact on and contribute to shaping a sun safety policy framework in the Netherlands.

\section{Support for sun safety policy}

Our findings present an important signal towards the Dutch government and politics to start with efforts towards policy change. Public acceptance or support for policy measures can positively influence the effectiveness of policy and plays an important role in the policy-making process. With this in mind, we explored support for sun safety policy in several small-scaled exploratory studies, not reported on in this thesis (unpublished data) among: 1) parents; 2) teachers and directors of primary schools; and 3) operators of recreational venues and policy makers. In short, parents seemed supportive of the (further) implementation of sun safety policy, and were especially positive about sunbed-related and school-related policies. Furthermore, while only a minority of teachers and school directors indicated that a sun safety policy was in place at their schools, they were supportive of integrating sun safety in their schools.

These preliminary findings indicate opportunities to implement sun safety policy at primary schools in the Netherlands. For example, educational programs addressing both children and their parents and a shade policy for school playgrounds should be developed and maintained. The Healthy School concept, aimed at promoting children's health at several levels of education, should ideally integrate UV-exposure as one of the health themes in their approach.

Lastly, when interviewing operators of recreational venues and policy officers at local and regional health authorities, both groups expressed moderate to high interest in skin cancer prevention efforts and recognized the need for the development and implementation of sun safety policies. These findings, based on interviews and surveys, albeit preliminary, demonstrate a 'window of opportunity' for sun safety policy among relevant target groups, thereby encouraging the creation of policy on a (Dutch) societal level.

\section{An integrative approach to climate-related health issues}

Time is all we have and don't have; the right time for addressing skin cancer in the Netherlands is now. Fortunately, initiatives are currently unfolded, recognizing the need for skin cancer prevention. National policy goals addressing climatological issues and heat stress were presented in the 'Nationale Klimaatadaptatiestrategie' (NAS) in 2018 (458). The aims described in this strategy were further translated in a research agenda from the Dutch fund for health research (ZonMw) (459). The excessively increased 
skin cancer incidence rates in the Netherlands were mentioned in this agenda, together with the call for efforts to reduce UVR-exposure on a societal level. In this ZonMw report, altering physical environments and thereby facilitating sun protection behaviours was acknowledged as an important strategy. We interpret this as a further opening up of the window of opportunity for the promotion of sun safety initiatives and advocate for the integration of strategies to tackle climate-related health problems - such as heat stress and put a stop to the increasing incidence of skin cancer in the Netherlands.

\section{A national 'call to action'}

Another national initiative that was recently established is the so-called 'Zonkracht Actieplan' (ZAP)(460), appointed by the Dutch Ministry of Health, Welfare and Sports. This collaboration platform serves as a 'call to action' to increase skin cancer awareness among the Dutch general public. This initiative unites all Dutch parties involved in skin cancer prevention, including both governmental and non-governmental foundations, universities and medical centres. The department of Health Promotion of Maastricht University has been actively involved in this task force since its establishment in 2017. Together with other experts from various fields, we share (scientific) knowledge, develop communication and strategy plans, formulate national sun safety recommendations, and specify research agendas for the upcoming years. Parts of the research presented in this thesis has already found its way and will continue to find its way into recommendation guidelines and strategic plans. Continued involvement in and contribution to the ZAP in the future will further ensure the translation of our scientific observations regarding primary skin cancer prevention into practical value.

\section{'Better by far to be good and courageous and bold and to make difference. Not change the world exactly, but the bit around you.'}

_ David Nicholls, One Day 


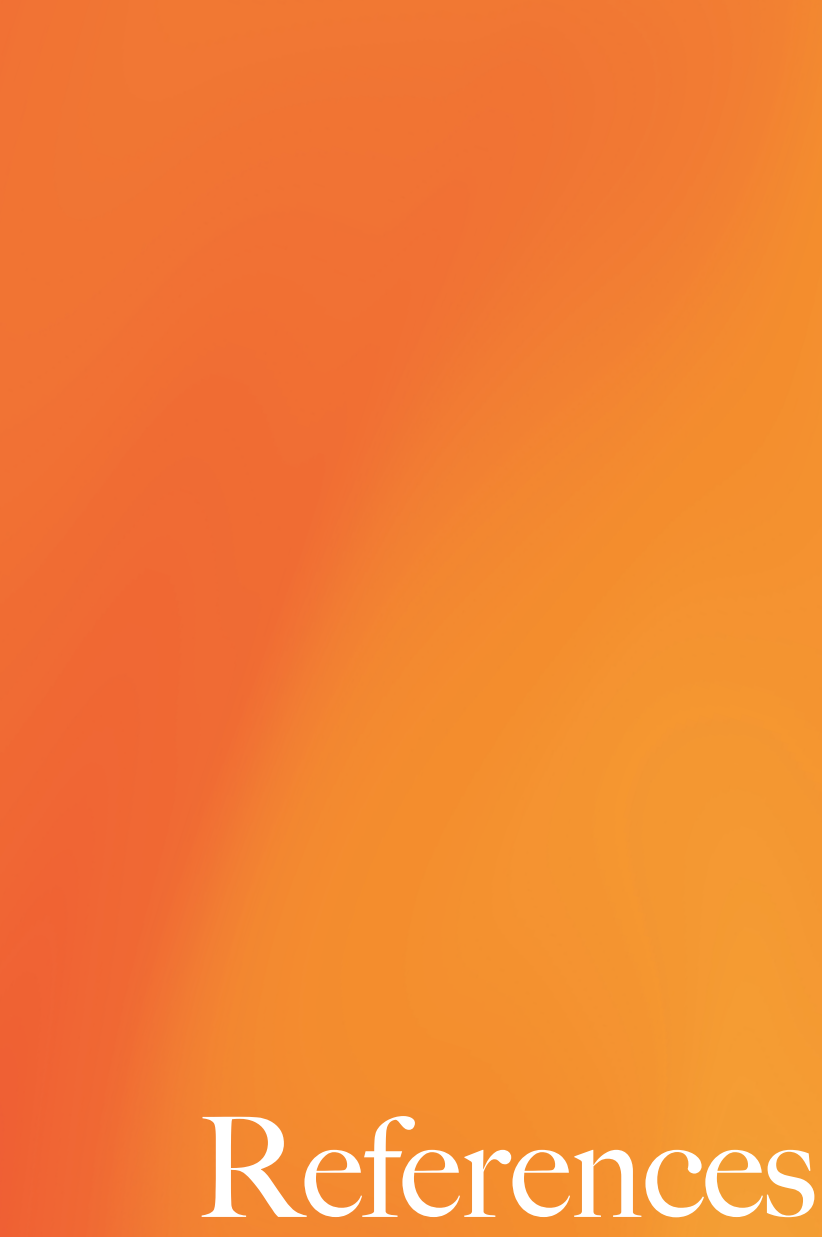
References

References 
1. Schadendorf D, Fisher DE, Garbe C, Gershenwald JE, Grob J-J, Halpern A, et al. Melanoma. Nature reviews Disease primers. 2015;1(1):1-20.

2. Karagas MR, Weinstock MA, Nelson HH. Keratinocyte carcinomas (basal and squamous cell carcinomas of the skin). In: Schottenfeld D, Fraumeni JF. Cancer epidemiology and prevention. Third edition. Oxford, UK: Oxford University Press; 2009. 3. Gilchrest BA, Eller MS, Geller AC, Yaar M. The pathogenesis of melanoma induced by ultraviolet radiation. N Engl J Med. 1999;340(17):1341-8.

4. Lai V, Cranwell W, Sinclair R. Epidemiology of skin cancer in the mature patient. Clin Dermatol. 2018;36(2):167-76.

5. Subramaniam P, Olsen CM, Thompson BS, Whiteman DC, Neale RE. Anatomical distributions of basal cell carcinoma and squamous cell carcinoma in a population-based study in Queensland, Australia. JAMA dermatol. 2017;153(2):175-82.

6. Watson M, Garnett E, Guy GP, Holman DM. The surgeon general's call to action to prevent skin cancer. Washington, DC: U.S. Department of Health and Human Services, Office of the Surgeon General; 2014.

7. Burton KA, Ashack KA, Khachemoune A. Cutaneous squamous cell carcinoma: a review of high-risk and metastatic disease. Am J Clin Dermatol. 2016;17(5):491-508.

8. Integraal Kankercentrum Nederland (IKNL). Huidkanker in Nederland. 2019.

9. Flohil SC, van der Leest RJ, Arends LR, de Vries E, Nijsten T. Risk of subsequent cutaneous malignancy in patients with prior keratinocyte carcinoma: a systematic review and meta-analysis. Eur J Cancer. 2013;49(10):2365-75.

10. Delavar A, Freedman DM, Velazquez-Kronen R, Little MP, Kitahara CM, Alexander BH, et al. Ultraviolet radiation and incidence of cataracts in a nationwide US cohort. Ophthalmic Epidemiol. 2018;25(5-6):403-11.

11. Slaper H, Van Dijk A, Den Outer P, van Kranen H, Slobbe L. UV-straling en gezondheid: Probleemveld en kennisbasis bij het RIVM. Bilthoven: Rijksinstituut voor Volksgezondheid en Milieu (RIVM); 2017.

12. Tobin DJ. Introduction to skin aging. J Tissue Viability. 2017;26(1):37-46.

13. Fitzmaurice C, Akinyemiju TF, Al Lami FH, Alam T, Alizadeh-Navaei R, Allen C, et al. Global, regional, and national cancer incidence, mortality, years of life lost, years lived with disability, and disability-adjusted life-years for 29 cancer groups, 1990 to 2016: a systematic analysis for the global burden of disease study. JAMA Oncol. 2018;4(11):1553-68.

14. Leiter U, Eigentler T, Garbe C. Epidemiology of skin cancer. Sunlight, vitamin D and skin cancer. New York, NY, US: Springer; 2014. p. 120-40.

15. Guy Jr GP, Thomas CC, Thompson T, Watson M, Massetti GM, Richardson LC. Vital signs: melanoma incidence and mortality trends and projections_United States, 1982-2030. MMWR Morb Mortal Wkly Rep. 2015;64(21):591.

16. Holterhues C, De Vries E, Louwman MW, Koljenović S, Nijsten T. Incidence and trends of cutaneous malignancies in the Netherlands, 1989-2005. J Invest Dermatol. 2010;130(7):1807-12.

17. Marcos-Gragera R, Vilar-Coromina N, Galceran J, Borràs J, Clèries R, Ribes J, et al. Rising trends in incidence of cutaneous malignant melanoma and their future projections in Catalonia, Spain: increasing impact or future epidemic? $\mathrm{J}$ Eur Acad Dermatol Venereol. 2010;24(9):1083-8.

18. Kohler BA, Sherman RL, Howlader N, Jemal A, Ryerson AB, Henry KA, et al. Annual report to the nation on the status of cancer, 1975-2011, featuring incidence of breast cancer subtypes by race/ethnicity, poverty, and state. J Natl Cancer Inst. 2015;107(6)

19. Matthews NH, Li WQ, Qureshi AA, Weinstock MA, Cho E. Epidemiology of Melanoma. In: Ward WH, Farma JM, editors. Cutaneous Melanoma: Etiology and Therapy. Brisbane, AU: Codon Publications; 2017. Chapter 1.

20. Karimkhani C, Green AC, Nijsten T, Weinstock MA, Dellavalle RP, Naghavi M, et al. The global burden of melanoma: results from the Global Burden of Disease Study 2015. Br J Dermatol. 2017;177(1):134-40.

21. Apalla Z, Nashan D, Weller RB, Castellsagué X. Skin Cancer: Epidemiology, Disease Burden, Pathophysiology, Diagnosis, and Therapeutic Approaches. Dermatol Ther (Heidelb). 2017;7(Suppl 1):5-19.

22. Khazaei Z, Ghorat F, Jarrahi A, Adineh H, Sohrabivafa M, Goodarzi E. Global incidence and mortality of skin cancer by histological subtype and its relationship with the human development index (HDI); an ecology study in 2018. World Cancer Res J. 2019;6(2):e13.

23. Verkouteren JAC, Ramdas KHR, Wakkee M, Nijsten T. Epidemiology of basal cell carcinoma: scholarly review. Br $J$ Dermatol. 2017;177(2):359-72.

24. van der Leest RJ, Flohil SC, Arends LR, de Vries E, Nijsten T. Risk of subsequent cutaneous malignancy in patients with prior melanoma: a systematic review and meta-analysis. J Eur Acad Dermatol Venereol. 2015;29(6):1053-62.

25. Volksgezondheidenzorg.info [Internet]. Bilthoven: RIVM; 2020 [cited 10 Nov 2020]. Available from: https://www. volksgezondheidenzorg.info/onderwerp/huidkanker/cijfers-context/trends\#methoden.

26. Bray F, Ferlay J, Soerjomataram I, Siegel RL, Torre LA, Jemal A. Global cancer statistics 2018: GLOBOCAN estimates of incidence and mortality worldwide for 36 cancers in 185 countries. CA Cancer J Clin. 2018;68(6):394-424.

27. Hollestein LM, van den Akker SA, Nijsten T, Karim-Kos HE, Coebergh JW, de Vries E. Trends of cutaneous melanoma in The Netherlands: increasing incidence rates among all Breslow thickness categories and rising mortality rates since 1989. Ann Oncol. 2012;23(2):524-30.

28. Sacchetto L, Zanetti R, Comber H, Bouchardy C, Brewster DH, Broganelli P, et al. Trends in incidence of thick, thin and in situ melanoma in Europe. Eur J Cancer. 2018;92:108-18.

29. EI Sharouni MA, Witkamp AJ, Sigurdsson V, van Diest PJ, Louwman MWJ, Kukutsch NA. Sex matters: men with melanoma have a worse prognosis than women. J Eur Acad Dermatol Venereol. 2019;33(11):2062-7.

30. Yang DD, Salciccioli JD, Marshall DC, Sheri A, Shalhoub J. Trends in malignant melanoma mortality in 31 countries from 1985 to 2015. Br J Dermatol. 2020. doi: 10.1111/bjd.19010

31. Simões MCF, Sousa JJS, Pais A. Skin cancer and new treatment perspectives: a review. Cancer Lett. 2015;357(1):8-42.

32. Cakir B, Adamson P, Cingi C. Epidemiology and economic burden of nonmelanoma skin cancer. Facial Plast Surg Clin North Am. 2012;20(4):419-22.

33. Guy GP, Jr., Machlin SR, Ekwueme DU, Yabroff KR. Prevalence and costs of skin cancer treatment in the U.S., $2002-2006$ 
and 2007-2011. Am J Prev Med. 2015;48(2):183-7.

34. Elliott TM, Whiteman DC, Olsen CM, Gordon LG. Estimated Healthcare Costs of Melanoma in Australia Over 3 Years Post-Diagnosis. Appl Health Econ Health Policy. 2017;15(6):805-16.

35. Fransen M, Karahalios A, Sharma N, English DR, Giles GG, Sinclair RD. Non-melanoma skin cancer in Australia. Med J Aust. 2012;197(10):565-8.

36. Volksgezondheidenzorg.info [Internet]. Bilthoven: RIVM; 2020. [cited 2 Nov 2020]. Available from: https://www. volksgezondheidenzorg.info/onderwerp/huidkanker/kosten/zorguitgaven\#!node-zorguitgaven-huidkanker-naar-sector.

37. Noels E, Hollestein L, Luijkx K, Louwman M, de Uyl-de Groot C, van den Bos R, et al. Increasing Costs of Skin Cancer due to Increasing Incidence and Introduction of Pharmaceuticals, 2007-2017. Acta Derm Venereol. 2020;100(10):adv00147.

38. van der Geer S, Reijers HA, van Tuijl HF, de Vries H, Krekels GA. Need for a new skin cancer management strategy. Arch Dermatol. 2010;146(3):332-6.

39. Noels E.C. Organisation of Skin Cancer Care Revisited [Internet]. Erasmus University Rotterdam; 2019. Available from: http://hdl.handle.net/1765/120017

40. International Agency for Research on Cancer. IARC Handbooks of Cancer Volume 5: sunscreens; 2001. Oxford, UK: Oxford University Press.

41. Elwood JM, Jopson J. Melanoma and sun exposure: an overview of published studies. Int J Cancer. 1997;73(2):198-203.

42. Whiteman DC, Whiteman CA, Green AC. Childhood sun exposure as a risk factor for melanoma: a systematic review of epidemiologic studies. Cancer Causes Control. 2001;12(1):69-82.

43. Green AC, Wallingford SC, McBride P. Childhood exposure to ultraviolet radiation and harmful skin effects: epidemiological evidence. Prog Biophys Mol Biol. 2011;107(3):349-55.

44. Gallagher RP, Hill GB, Bajdik CD, Fincham S, Coldman AJ, McLean DI, et al. Sunlight exposure, pigmentary factors, and risk of nonmelanocytic skin cancer. I. Basal cell carcinoma. Arch Dermatol. 1995;131(2):157-63.

45. Armstrong BK, Kricker A. The epidemiology of UV induced skin cancer. J Photochem Photobiol B. 2001;63(1-3):8-18.

46. Volkmer B, Greinert R. UV and children's skin. Prog Biophys Mol Biol. 2011;107(3):386-8.

47. Cestari T, Buster K. Photoprotection in specific populations: Children and people of color. J Am Acad Dermatol. 2017;76(3s1):S110-s21.

48. Ackermann S, Vuadens A, Levi F, Bulliard JL. Sun protective behaviour and sunburn prevalence in primary and secondary schoolchildren in western Switzerland. Swiss Med Wkly. 2016;146:w14370.

49. Dusza SW, Halpern AC, Satagopan JM, Oliveria SA, Weinstock MA, Scope A, et al. Prospective study of sunburn and sun behavior patterns during adolescence. Pediatrics. 2012;129(2):309-17.

50. Hall HI, McDavid K, Jorgensen CM, Kraft JM. Factors associated with sunburn in white children aged 6 months to 11 years. Am J Prev Med. 2001;20(1):9-14.

51. O'Riordan DL, Geller AC, Brooks DR, Zhang Z, Miller DR. Sunburn reduction through parental role modeling and sunscreen vigilance. J Pediatr. 2003;142(1):67-72.

52. Behrens CL, Thorgaard C, Philip A, Bentzen J. Sunburn in children and adolescents: associations with parents' behaviour and attitudes. Scand J Public Health. 2013;41(3):302-10

53. Wu YP, Parsons BG, Aspinwall LG, Hay JL, Boucher KM, Caputo $H$, et al. Parent and child perspectives on perceived barriers to child sun protection and their association with sun protection strategies among children of melanoma survivors. Pediatr Dermatol. 2019;36(3):317-23.

54. Boog MC, Nederend A, Ultee J. Sun Exposure and Sun Protection at Primary Schools in The Netherlands: A CrossSectional Study. Pediatr Dermatol. 2016;33(1):38-43.

55. Thoonen K, van Osch L, Crutzen R, De Vries H, Schneider F. Identification of relevant socio-cognitive determinants explaining multiple parental sun protection behaviors [preprint] 2020. Available from: https://psyarxiv.com/yuqzd/. doi: 10.31234/osf.io/yuqzd.

56. Thoonen K, Lima Passos V, Schneider F, de vries H, Van Osch L. Children's sunburn exposed: Identification of sun exposure and parental sun protection patterns. Eur

J Derma. Forthcoming 2021. Available from: https://psyarxiv.com/fcz5b/. doi [preprint]: 10.31234/osf.io/fcz5b.

57. Gordon R. Skin cancer: an overview of epidemiology and risk factors. Semin Oncol Nurs. 2013;29(3):160-9.

58. Stern RS, Weinstein MC, Baker SG. Risk reduction for nonmelanoma skin cancer with childhood sunscreen use. Arch Dermatol. 1986;122(5):537-45.

59. Gandini S, Sera F, Cattaruzza MS, Pasquini P, Picconi O, Boyle P, et al. Meta-analysis of risk factors for cutaneous melanoma: II. Sun exposure. Eur J Cancer. 2005;41(1):45-60.

60. Dailey CP. Teaching parents and children preventive health behaviors. Fam Community Health. 1985; 7(4), 34-43. doi: 10.1097/00003727-198502000-00007.

61. Telama R, Yang X, Viikari J, Välimäki I, Wanne O, Raitakari O. Physical activity from childhood to adulthood: a 21-year tracking study. Am J Prev Med. 2005;28(3):267-73.

62. Gandini S, Sera F, Cattaruzza MS, Pasquini P, Zanetti R, Masini C, et al. Meta-analysis of risk factors for cutaneous melanoma: III. Family history, actinic damage and phenotypic factors. Eur J Cancer. 2005;41(14):2040-59.

63. Belbasis L, Stefanaki I, Stratigos AJ, Evangelou E. Non-genetic risk factors for cutaneous melanoma and keratinocyte skin cancers: An umbrella review of meta-analyses. J Dermatol Sci. 2016;84(3):330-9.

64. Gandhi SA, Kampp J. Skin Cancer Epidemiology, Detection, and Management. Med Clin North Am. 2015;99(6):1323-35.

65. Lens MB, Dawes M. Global perspectives of contemporary epidemiological trends of cutaneous malignant melanoma. Br J Dermatol. 2004;150(2):179-85.

66. Olsen CM, Wilson LF, Green AC, Biswas N, Loyalka J, Whiteman DC. How many melanomas might be prevented if more people applied sunscreen regularly? Br J Dermatol. 2018;178(1):140-7.

67. Niederhuber JE, Armitage JO, Doroshow JH, Kastan MB, Tepper JE. Abeloff's Clinical Oncology. E-Book: Elsevier Health 
Sciences; 2013.

68. Association of European Cancer Leagues. EC: Sun Safety Recommendations [Internet]. Brussels, BE: Association of European Cancer Leagues; 2020 [Cited 1 Oct 2020]. Available from: https://www.europeancancerleagues.org/sun-safetyrecommendations/.

69. Centers for Disease Control and Prevention. Sun Safety [Internet]. Atlanta, GA, US: Centers for Disease Control and Prevention; 2020. [cited 2 Sept 2020]. Available from: https://www.cdc.gov/cancer/skin/basic_info/sun-safety.htm.

70. American Cancer Society. How do I protect myself from Ultraviolet (UV) rays? [Internet]. Atlanta, GA, US: American Cancer Society; 2019. [cited 20 Aug 2020]. Available from: https://www.cancer.org/healthy/be-safe-in-sun/uv-protection. html.

71. Hill D, Dixon H. Promoting sun protection in children: rationale and challenges. Health Educ Behav. 1999;26(3):409-17.

72. Berneburg M, Surber C. Children and sun protection. Br J Dermatol. 2009;161 Suppl 3:33-9.

73. Dutch Cancer Society. Zonbescherming voor kinderen [Internet]. Amsterdam; KWF Kankerbestrijding; 2019. [cited 1 Oct 2020]. Available from: https://www.kwf.nl/kanker-voorkomen/zon-uv-straling-en-huidkanker/zonnetips.

74. Tan MG, Nag S, Weinstein M. Parental use of sun protection for their children-does skin color matter? Pediatr Dermatol. 2018;35(2):220-4.

75. Johnson K, Davy L, Boyett T, Weathers L, Roetzheim RG. Sun protection practices for children: knowledge, attitudes, and parent behaviors. Arch Pediatr Adolesc Med. 2001;155(8):891-6.

76. Klostermann S, Bolte G. Determinants of inadequate parental sun protection behaviour in their children--results of a cross-sectional study in Germany. Int J Hyg Environ Health. 2014;217(2-3):363-9.

77. Dadlani C, Orlow SJ. Planning for a brighter future: a review of sun protection and barriers to behavioral change in children and adolescents. Dermatol Online J. 2008;14(9):1.

78. Gefeller O, Uter W, Pfahlberg AB. Protection from Ultraviolet Radiation during Childhood: The Parental Perspective in Bavaria. Int J Environ Res Public Health. 2016;13(10).

79. Stanton WR, Janda M, Baade PD, Anderson P. Primary prevention of skin cancer: a review of sun protection in Australia and internationally. Health Promot Int. 2004;19(3):369-78.

80. Dobbinson S, Wakefield M, Hill D, Girgis A, Aitken JF, Beckmann K, et al. Children's sun exposure and sun protection: prevalence in Australia and related parental factors. J Am Acad Dermatol. 2012;66(6):938-47.

81. Reinau D, Meier C, Gerber N, Hofbauer GF, Surber C. Sun protective behaviour of primary and secondary school students in North-Western Switzerland. Swiss Med Wkly. 2012;142:w13520.

82. Saraiya M, Glanz K, Briss PA, Nichols P, White C, Das D, et al. Interventions to prevent skin cancer by reducing exposure to ultraviolet radiation: a systematic review. Am J Prev Med. 2004;27(5):422-66.

83. Goldthorpe J, Epton T, Keyworth C, Calam R, Armitage C. Who is responsible for keeping children healthy? A qualitative exploration of the views of children aged 8-10 years old. BMJ Open. 2019;9(5):e025245.

84. Lewis CE, Lewis MA. Improving the health of children: must the children be involved? Annu Rev Public Health. 1983;4:25983.

85. Gubbels JS, Kremers SP, Stafleu A, de Vries SI, Goldbohm RA, Dagnelie PC, et al. Association between parenting practices and children's dietary intake, activity behavior and development of body mass index: the KOALA Birth Cohort Study. Int $J$ Behav Nutr Phys Act. 2011;8:18.

86. Castilho AR, Mialhe FL, Barbosa Tde S, Puppin-Rontani RM. Influence of family environment on children's oral health: a systematic review. J Pediatr (Rio J). 2013;89(2):116-23.

87. Kremers SP, Brug J, de Vries H, Engels RC. Parenting style and adolescent fruit consumption. Appetite. 2003;41(1):43-50 88. $\mathrm{Xu} \mathrm{H}$, Wen LM, Rissel C. Associations of parental influences with physical activity and screen time among young children: a systematic review. J Obes. 2015;2015:546925.

89. Li H, Colantonio S, Dawson A, Lin X, Beecker J. Sunscreen Application, Safety, and Sun Protection: The Evidence. J Cutan Med Surg. 2019;23(4):357-69.

90. Cooley JH, Quale LM. Skin cancer preventive behavior and sun protection recommendations. Semin Oncol Nurs. 2013;29(3):223-6.

91. Diao DY, Lee TK. Sun-protective behaviors in populations at high risk for skin cancer. Psychol Res Behav Manag. 2013;7:918.

92. Gies P, Roy C, Toomey S, MacLennan R, Watson M. Solar UVR exposures of primary school children at three locations in Queensland. Photochem Photobiol. 1998;68(1):78-83.

93. Vanos JK. Children's health and vulnerability in outdoor microclimates: A comprehensive review. Environ Int. 2015;76:1-15. 94. Lucas RM, Neale RE, Madronich S, McKenzie RL. Are current guidelines for sun protection optimal for health? Exploring the evidence. Photochem Photobiol Sci. 2018;17(12):1956-63.

95. Gallagher RP, Lee TK. Adverse effects of ultraviolet radiation: a brief review. Prog Biophys Mol Biol. 2006;92(1):119-31.

96. Balk SJ. Ultraviolet radiation: a hazard to children and adolescents. Pediatrics. 2011;127(3):e791-817.

97. Coelho SG, Choi W, Brenner M, Miyamura Y, Yamaguchi Y, Wolber R, et al. Short- and long-term effects of UV radiation on the pigmentation of human skin. J Investig Dermatol Symp Proc. 2009;14(1):32-5.

98. Fitch-Martin AR, Menger LM, Loomis AD, Hartsough LE, Henry KL. "We Don't Really Do Anything Unless it's Really Bad": Understanding Adolescent Sun Protective Knowledge, Attitudes and Behaviors in the US. J Prim Prev. 2018;39(4):371-86.

99. Aleo G, Bagnasco A, Cozzani E, Parodi A, CannavÒ SP, Watson R, et al. Sun-safe behaviours, personal risk, level of concern, and knowledge about cutaneous melanoma in italy: time for social marketing? J Prev Med Hyg. 2020;61(2):E246-e58.

100. Venning VL, Abbott LM, Thomas CL, Fernandez-Peñas P. Risk Perception Plays Minimal Role in Sun Exposure Behaviours. J Cancer Educ. 2020;35(1):125-30.

101. Lorenc T, Jamal F, Cooper C. Resource provision and environmental change for the prevention of skin cancer: systematic review of qualitative evidence from high-income countries. Health Promot Int. 2013;28(3):345-56. 
102. Garside R, Pearson M, Moxham T. What influences the uptake of information to prevent skin cancer? A systematic review and synthesis of qualitative research. Health Educ Res. 2010;25(1):162-82.

103. Mead MN. Benefits of sunlight: a bright spot for human health. Environ Health Perspect. 2008;116(4):A160-7.

104. Robinson JK. Sun exposure, sun protection, and vitamin D. JAMA. 2005;294(12):1541-3.

105. Stalgis-Bilinski KL, Boyages J, Salisbury EL, Dunstan CR, Henderson SI, Talbot PL. Burning daylight: balancing vitamin $D$ requirements with sensible sun exposure. Med J Aust. 2011;194(7):345-8.

106. Antonucci R, Locci C, Clemente MG, Chicconi E, Antonucci L. Vitamin D deficiency in childhood: old lessons and current challenges. J Pediatr Endocrinol Metab. 2018;31(3):247-60.

107. Draelos ZD. The allure of tanned skin. J Cosmet Dermatol. 2009;8(1):1-2

108. Yoo J-J, Kim H-Y. Perceived negative health effect of tanning: the interface between tanning attitudes and behaviors. Cloth Text Res J. 2014;32(1):6-19.

109. Glanz K, Rimer BK, Viswanath K. Health behavior: Theory, research, and practice, 5th ed. San Francisco, CA, US: JosseyBass; 2015.

110. Bartholomew Eldredge LK, Markham CM, Ruiter RA, Fernández ME, Kok G, Parcel GS. Planning health promotion programs: an intervention mapping approach, 4th ed. San Francisco, CA, US: Jossey-Bass; 2016.

111. Gielen AC, McDonald EM, Gary TL, Bone LR. Using the precede-proceed model to apply health behavior theories. In: Glanz K, Rimer BK, Viswanath K, editors. Health behavior and health education: Theory, research, and practice, 4th ed. San Francisco, CA, US: Jossey-Bass; 2008. Chapter 4, p. 407-29.

112. Sallis JF, Owen N, Fisher E. Ecological models of health behavior. In: Glanz K, Rimer BK, Viswanath K, editors. Health behavior: Theory, research, and practice, 5th ed. San Francisco, CA, US: Jossey-Bass; 2015. Chapter 3, p. 43-64.

113. Hagger MS. Non-conscious processes and dual-process theories in health psychology. Health Psychology Review. 2016; $11(1), 100$.

114. Gefeller O, Uter W, Pfahlberg AB. Good, but Not Perfect: Parental Knowledge About Risk Factors for Skin Cancer and the Necessity of Sun Protection in Southern Germany. Pediatr Dermatol. 2015;32(4):e159-60.

115. Met Office. Research highlights parents' relaxed attitude to sun safety [Internet]. Exeter, UK; Met Office; 2017 . [cited 1 Oct 2020]. Available from: https://www.metoffice.gov.uk/about-us/press-office/news/weather-and-climate/2017/researchhighlights-parents-relaxed-attitude-to-sun-safety.

116. Li J, Uter W, Pfahlberg A, Gefeller O. Parental perspective on sun protection for young children in Bavaria. Photodermatol Photoimmunol Photomed. 2011;27(4):196-202.

117. Cercato MC, Nagore E, Ramazzotti V, Sperduti I, Guillén C. Improving sun-safe knowledge, attitude and behaviour in parents of primary school children: a pilot study. J Cancer Educ. 2013;28(1):151-7.

118. Thomson CE, White KM, Hamilton K. Investigating mothers' decisions about their child's sun-protective behaviour using the Theory of Planned Behaviour. J Health Psychol. 2012;17(7):1001-10.

119. Turner LR, Mermelstein RJ. Psychosocial characteristics associated with sun protection practices among parents of young children. J Behav Med. 2005;28(1):77-90.

120. Buller DB, Cokkinides V, Hall HI, Hartman AM, Saraiya M, Miller E, et al. Prevalence of sunburn, sun protection, and indoor tanning behaviors among Americans: review from national surveys and case studies of 3 states. J Am Acad Dermatol. 2011;65(5 Suppl 1):S114-23.

121. Noar SM, Benac CN, Harris MS. Does tailoring matter? Meta-analytic review of tailored print health behavior change interventions. Psychol Bull. 2007;133(4):673-93.

122. Glanz K, Volpicelli K, Jepson C, Ming ME, Schuchter LM, Armstrong K. Effects of tailored risk communications for skin cancer prevention and detection: the PennSCAPE randomized trial. Cancer Epidemiol Biomarkers Prev. 2015;24(2):415-21.

123. Geller AC, Jablonski NG, Pagoto SL, Hay JL, Hillhouse J, Buller DB, et al. Interdisciplinary Perspectives on Sun Safety. JAMA Dermatol. 2018;154(1):88-92.

124. Ajzen I, Albarracín D. Predicting and changing behavior: A reasoned action approach. In: Ajzen I, Albarracín D, Hornik $R$, editors. Prediction and change of health behavior: Applying the reasoned action approach. New Jersey, US: Lawrence Erlbaum Associates Publishers; 2007, p. 3-21.

125. Armitage CJ, Conner M. Social cognition models and health behaviour: A structured review. Psychol Health 2000;15(2):173-89.

126. Milne S, Sheeran P, Orbell S. Prediction and intervention in health-related behavior: A meta-analytic review of protection motivation theory. J Appl Soc Psychol. 2000;30(1):106-43.

127. Starfelt Sutton LC, White KM. Predicting sun-protective intentions and behaviours using the theory of planned behaviour: a systematic review and meta-analysis. Psychol Health. 2016;31(11):1272-92.

128. Allom V, Mullan B, Sebastian J. Closing the intention-behaviour gap for sunscreen use and sun protection behaviours. Psychol Health. 2013;28(5):477-94.

129. Myers LB, Horswill MS. Social cognitive predictors of sun protection intention and behavior. Behav Med. 2006;32(2):5763.

130. Hamilton K, Kirkpatrick A, Rebar A, White KM, Hagger MS. Protecting young children against skin cancer: Parental beliefs, roles, and regret. Psychooncology. 2017;26(12):2135-41.

131. Hamilton K, Kirkpatrick A, Rebar A, Hagger MS. Child sun safety: Application of an Integrated Behavior Change model. Health Psychol. 2017;36(9):916-26.

132. de Vries H, van Osch L, Eijmael K, Smerecnik C, Candel M. The role of risk perception in explaining parental sunscreen use. Psychol Health. 2012;27(11):1342-58.

133. Van Osch L, Reubsaet A, Lechner L, Candel M, Mercken L, De Vries H. Predicting parental sunscreen use: Disentangling the role of action planning in the intention-behavior relationship. Psychol Health. 2008;23(7):829-47.

134. Alves SAA, de Oliveira MLB. Sociocultural aspects of health and disease and their pragmatic impact. J Human Growth 
Dev. 2018;28(2):183-8.

135. Noar SM, Zimmerman RS. Health behavior theory and cumulative knowledge regarding health behaviors: are we moving in the right direction? Health Educ Res. 2005;20(3):275-90.

136. Buller DB, Borland R. Skin cancer prevention for children: a critical review. Health Educ Behav. 1999;26(3):317-43.

137. Montague M, Borland R, Sinclair C. Slip! Slop! Slap! and SunSmart, 1980-2000: Skin cancer control and 20 years of population-based campaigning. Health Educ Behav. 2001;28(3):290-305.

138. Tabbakh T, Volkov A, Wakefield M, Dobbinson S. Implementation of the SunSmart program and population sun protection behaviour in Melbourne, Australia: Results from cross-sectional summer surveys from 1987 to 2017. PLoS Med. 2019;16(10):e1002932.

139. Hill D, Dobbinson S, Makin JK. Interventions to lower ultraviolet radiation exposure: education, legislation, and public policy [Internet]. In: ASCO 2009 Education Book. Alexandria, VA, US: American Society of Clinical Oncology; 2009. p. 526531. Available from: https://www.researchgate.net/profile/Jennifer_Makin/publication/281981574_Interventions_to_lower_ ultraviolet_radiation_exposure_Education_legislation_and_public_policy/links/561c8b3d08ae6d17308b1a98.pdf.

140. Jones SB, Beckmann K, Rayner J. Australian primary schools' sun protection policy and practice: evaluating the impact of the National SunSmart Schools Program. Health Promot J Austr. 2008;19(2):86-90.

141. Mayer JA, Lewis EC, Eckhardt L, Slymen D, Belch G, Elder J, et al. Promoting sun safety among zoo visitors. Prev Med. 2001;33(3):162-9.

142. Boldemann C, Dal H, Mårtensson F, Cosco N, Moore R, Bieber B, et al. Preschool outdoor play environment may combine promotion of children's physical activity and sun protection. Further evidence from Southern Sweden and North Carolina. Sci Sports. 2011;26(2):72-82.

143. Rodrigues A, Sniehotta FF, Araujo-Soares V. Are interventions to promote sun-protective behaviors in recreational and tourist settings effective? A systematic review with meta-analysis and moderator analysis. Ann Behav Med. 2013;45(2):22438.

144. Holman DM, Kapelos GT, Shoemaker M, Watson M. Shade as an Environmental Design Tool for Skin Cancer Prevention. Am J Public Health. 2018;108(12):1607-12.

145. Hagger MS, Cameron LD, Hamilton K, Hankonen N, Lintunen T. The handbook of behavior change. Cambridge, UK: Cambridge University Press, 2020.

146. Apalla Z, Lallas A, Sotiriou E, Lazaridou E, loannides D. Epidemiological trends in skin cancer. Dermatol Pract Concept. 2017;7(2):1-6.

147. Rigel DS, Russak J, Friedman R. The evolution of melanoma diagnosis: 25 years beyond the ABCDs. CA Cancer J Clin. 2010;60(5):301-16

148. McPherson M, Elwood M, English DR, Baade PD, Youl PH, Aitken JF. Presentation and detection of invasive melanoma in a high-risk population. J Am Ac Dermatol. 2006;54(5):783-92.

149. Erdmann F, Lortet-Tieulent J, Schüz J, Zeeb H, Greinert R, Breitbart EW, et al. International trends in the incidence of malignant melanoma 1953-2008 - are recent generations at higher or lower risk? Int J Cancer. 2013;132(2):385-400.

150. Gordon LG, Rowell D. Health system costs of skin cancer and cost-effectiveness of skin cancer prevention and screening: a systematic review. Eur J Cancer Prev. 2015;24(2):141-9.

151. Pil L, Hoorens I, Vossaert K, Kruse V, Tromme I, Speybroeck N, et al. Burden of skin cancer in Belgium and costeffectiveness of primary prevention by reducing ultraviolet exposure. Prev Med. 2016;93:177-82.

152. Markovic SN, Erickson LA, Rao RD, Weenig RH, Pockaj BA, Bardia A, et al. Malignant melanoma in the 21st century, part 1: epidemiology, risk factors, screening, prevention, and diagnosis. Mayo Clin Proc. 2007;82(3):364-80.

153. Oliveria SA, Saraiya M, Geller AC, Heneghan MK, Jorgensen C. Sun exposure and risk of melanoma. Arch Dis Child. 2006;91(2):131-8.

154. Day AK, Stapleton JL, Natale-Pereira AM, Goydos JS, Coups EJ. Parent and Child Characteristics Associated with Child Sunburn and Sun Protection Among U.S. Hispanics. Pediatr Dermatol. 2017;34(3):315-21.

155. Marks R. Skin cancer - childhood protection affords lifetime protection. Med J Aust. 1987;147(10):475-6.

156. Thoonen K, Schneider F, Candel M, de Vries H, van Osch L. Childhood sun safety at different ages: relations between parental sun protection behavior towards their child and children's own sun protection behavior. BMC Public Health. 2019;19(1):1044.

157. Turrisi R, Hillhouse J, Robinson J, Stapleton J, Adams M. Influence of parent and child characteristics on a parent-based intervention to reduce unsafe sun practices in children 9 to 12 years old. JAMA Dermatol. 2006;142(8):1009-14.

158. Bellamy R. A systematic review of educational interventions for promoting sun protection knowledge, attitudes and behaviour following the QUESTS approach. Med Teach. 2005;27(3):269-75.

159. Hart KM, DeMarco RF. Primary prevention of skin cancer in children and adolescents: a review of the literature. $J$ Pediatr Oncol. 2008;25(2):67-78.

160. Weinstein JM, Yarnold PR, Hornung RL. Parental knowledge and practice of primary skin cancer prevention: gaps and solutions. Pediatr Dermatol. 2001;18(6):473-7.

161. Gefeller O, Li J, Uter W, Pfahlberg AB. The impact of parental knowledge and tanning attitudes on sun protection practice for young children in Germany. Int J Environ Res Public Health. 2014;11(5):4768-81.

162. De Vries H. An integrated approach for understanding health behavior: The I-Change Model as an example. Int $J$ Psychol Behav Sci. 2017;2(2).

163. Littlewood Z, Greenfield S. Parents' knowledge, attitudes and beliefs regarding sun protection in children: a qualitative study. BMC public health. 2018;18(1):1-11.

164. Bargh JA, Chartrand TL. The unbearable automaticity of being. Am Psychol. 1999;54(7):462.

165. Kremers SP, de Bruijn GJ, Visscher TL, van Mechelen W, de Vries NK, Brug J. Environmental influences on energy balance-related behaviors: a dual-process view. The international journal of behavioral nutrition and physical activity. 
2006;3:9.

166. Thoonen K, Osch LV, Vries H, Jongen S, Schneider F. Are Environmental Interventions Targeting Skin Cancer Prevention among Children and Adolescents Effective? A Systematic Review. Int J Behav Nutr Phys Act. 2020;17(2).

167. Parisi AV, Turnbull DJ. Shade provision for UV minimization: a review. Photochem Photobiol. 2014;90(3):479-90.

168. Schneider S, Bolbos A, Kadel P, Holzwarth B. Exposed children, protected parents; shade in playgrounds as a previously unstudied intervention field of cancer prevention. Int J Environ Health Res. 2020;30(1):26-37.

169. Vanos JK, McKercher GR, Naughton K, Lochbaum M. Schoolyard Shade and Sun Exposure: Assessment of Personal Monitoring During Children's Physical Activity. Photochem Photobiol. 2017;93(4):1123-32.

170. Glenn BA, Lin T, Chang LC, Okada A, Wong WK, Glanz K, et al. Sun protection practices and sun exposure among children with a parental history of melanoma. Cancer Epidemiol Biomarkers Prev. 2015;24(1):169-77.

171. Rapport F, Hogden A, Faris M, Bierbaum M, Clay-Williams R, Long J, et al. Qualitative research in healthcare: modern methods, clear translation: a white paper [Internet]. Sydney, AU: Australian Institute of Health Innovation, Macquarie University; 2018. Available from: https://research-management.mq.edu.au/ws/portalfiles/portal/92373203/White_ Paper_28.08.18_FINAL.pdf

172. Farooq MB, De Villiers $C$. Telephonic qualitative research interviews: When to consider them and how to do them. Meditari Acc Res. 2017;25(2): 291-316

173. D'Onofrio $\mathrm{CN}$. The use of self-reports on sensitive behaviors in health program evaluation. New Dir Progr Ev. 1989;1989(43):59-74.

174. Scheidt PC, Brenner RA, Rossi MW, Clyman R, Boyle KE. Parental attitudes regarding interviews about injuries to their children. Inj Prev. 2000;6(1):51-5.

175. Tong A, Sainsbury P, Craig J. Consolidated criteria for reporting qualitative research (COREQ): a 32-item checklist for interviews and focus groups. Int J Qual Health Care. 2007;19(6):349-57.

176. Palinkas LA, Horwitz SM, Green CA, Wisdom JP, Duan N, Hoagwood K. Purposeful sampling for qualitative data collection and analysis in mixed method implementation research. Adm Policy Ment Health. 2015;42(5):533-44.

177. Qualtrics. Provo, UT, USA. Version 2019. Available from: https://www.qualtrics.com.

178. Robinson OC. Sampling in interview-based qualitative research: A theoretical and practical guide. Qual Res Psychol. 2014;11(1):25-41.

179. Park TL, Adams SG, Lynch J. Sociodemographic factors in Health Psychology research: 12 years in review. Health Psychol. 1998;17(4):381-3.

180. Thyer BA. The handbook of social work research methods, 2nd ed. Los Angeles, CA, US: Sage Publications; 2009.

181. Carter N, Bryant-Lukosius D, DiCenso A, Blythe J, Neville AJ. The use of triangulation in qualitative research. Oncol Nurs Forum. 2014;41(5):545-7.

182. Phillippi J, Lauderdale J. A Guide to Field Notes for Qualitative Research: Context and Conversation. Qual Health Res. 2018;28(3):381-8.

183. Saunders B, Sim J, Kingstone T, Baker S, Waterfield J, Bartlam B, et al. Saturation in qualitative research: exploring its conceptualization and operationalization. Qual Quant. 2018;52(4):1893-907.

184. Fitzpatrick TB. The validity and practicality of sun-reactive skin types I through VI. Arch Dermatol. 1988;124(6):869-71.

185. NVivo Qualitative Data Analysis Software. QSR International Pty Ltd. Version 12, 2018.

186. Braun V, Clarke V. Using thematic analysis in psychology. Qual Res Psychol. 2006;3(2):77-101.

187. McHugh ML. Interrater reliability: the kappa statistic. Biochem Med. 2012;22(3):276-82.

188. Sun S. Meta-analysis of Cohen's kappa. Health Serv Outcomes Res Methodol. 2011;11(3-4):145-63.

189. Tripp MK, Diamond PM, Vernon SW, Swank PR, Dolan Mullen P, Gritz ER. Measures of parents' self-efficacy and perceived barriers to children's sun protection: construct validity and reliability in melanoma survivors. Health Educ Res. 2013;28(5):828-42.

190. Dennis LK, Lowe JB, Snetselaar LG. Tanning behavior among young frequent tanners is related to attitudes and not lack of knowledge about the dangers. Health Educ J. 2009;68(3):232-43.

191. Kirk L, Greenfield S. Knowledge and attitudes of UK university students in relation to ultraviolet radiation (UVR) exposure and their sun-related behaviours: a qualitative study. BMJ Open. 2017;7(3):e014388.

192. White KM, Starfelt LC, Young RM, Hawkes AL, Leske S, Hamilton K. Predicting A ustralian adults' sun-safe behaviour: Examining the role of personal and social norms. Br J Health Psychol. 2015;20(2):396-412.

193. Autier P, Boniol M, Doré JF. Sunscreen use and increased duration of intentional sun exposure: still a burning issue. Int J Cancer. 2007;121(1):1-5.

194. Linos E, Keiser E, Fu T, Colditz G, Chen S, Tang JY. Hat, shade, long sleeves, or sunscreen? Rethinking US sun protection messages based on their relative effectiveness. Cancer Causes Control. 2011;22(7):1067-71.

195. Robinson JK, Rigel DS, Amonette RA. Summertime sun protection used by adults for their children. J Am Acad Dermatol. 2000;42(5 Pt 1):746-53.

196. Bränström R, Kasparian NA, Chang YM, Affleck P, Tibben A, Aspinwall LG, et al. Predictors of sun protection behaviors and severe sunburn in an international online study. Cancer Epidemiol Biomarkers Prev. 2010;19(9):2199-210.

197. Kok G, Gottlieb NH, Peters GJ, Mullen PD, Parcel GS, Ruiter RA, et al. A taxonomy of behaviour change methods: an Intervention Mapping approach. Health Psychol Rev. 2016;10(3):297-312.

198. Shoveller JA, Lovato CY. Measuring self-reported sunburn: challenges and recommendations. Chronic Dis Can. 2001;22(3-4):83-98.

199. Naylor M, Robinson JK. Sunscreen, sun protection, and our many failures. Arch Dermatol. 2005;141(8):1025-7.

200. Whiting LS. Semi-structured interviews: guidance for novice researchers. Nurs Stand. 2008;22(23):35-40.

201. Seale C, Gobo G, Gubrium JF, Silverman D. Qualitative Research Practice. Los Angeles, CA, US: Sage; 2004.

202. Novick $G$. Is there a bias against telephone interviews in qualitative research? Res Nurs Health. 2008;31(4):391-8. 
203. Bränström R, Kristjansson S, Dal H, Rodvall Y. Sun exposure and sunburn among Swedish toddlers. Eur J Cancer 2006;42(10):1441-7.

204. Althubaiti A. Information bias in health research: definition, pitfalls, and adjustment methods. J Multidiscip Healthc 2016;9:211-7.

205. Smith A, Harrison S, Nowak M, Buettner P, Maclennan R. Changes in the pattern of sun exposure and sun protection in young children from tropical Australia. J Am Acad Dermatol. 2013;68(5):774-83.

206. O'Riordan DL, Stanton WR, Eyeson-Annan M, Gies P, Roy C. Correlations between reported and measured ultraviolet radiation exposure of mothers and young children. Photochem Photobiol. 2000;71(1):60-4.

207. International Agency for Research on Cancer. The Global Cancer Observatory (GLOBOCAN). Melanoma of Skin Factsheet [Internet]. Lyon, FR: IARC, 2018. [cited 1 Aug 2020]. Available from: https://gco.iarc.fr/today/data/factsheets/ cancers/16-Melanoma-of-skin-fact-sheet.pdf.

208. Whiteman DC, Green AC, Olsen CM. The Growing Burden of Invasive Melanoma: Projections of Incidence Rates and Numbers of New Cases in Six Susceptible Populations through 2031. J Invest Dermatol. 2016;136(6):1161-71.

209. Arnold M, de Vries E, Whiteman DC, Jemal A, Bray F, Parkin DM, et al. Global burden of cutaneous melanoma attributable to ultraviolet radiation in 2012. Int J Cancer. 2018;143(6):1305-14.

210. Hagger MS, Hamilton K. Health behavior, health promotion, and the transition to parenthood: Insights from research in health psychology and behavior change. In: Taubman BA. Pathways and Barriers to Parenthood. New York, NY, UA: Springer International Publishing; 2019. p. 251-69.

211. Buller DB, Callister MA, Reichert T. Skin cancer prevention by parents of young children: health information sources, skin cancer knowledge, and sun-protection practices. Oncol Nurs Forum. 1995;22(10):1559-66.

212. The Skin Cancer Foundation. Prevention Guidelines [Internet]. New York, NY, US: The Skin Cancer Foundation; 2020. [cited 1 May 2020]. Available from: http://www.skincancer.org/prevention/sun-protection/prevention-guidelines.

213. American Association of Dermatologists. Sunscreen FAQ [Internet]. Rosemont, IL, US: 2019. [cited 1 May 2020]. Available from: https://assets.ctfassets.net/1ny4yoiyrqia/4xAHF2HbKPsmHnLOCTZcFS/b5e2a00d2376dc596088f764e8a1fOfO/ Sunscreen_FAQ_5-19.pdf.

214. Koch S, Pettigrew S, Strickland M, Slevin T, Minto C. Sunscreen Increasingly Overshadows Alternative Sun-Protection Strategies. J Cancer Educ. 2017;32(3):528-31.

215. Autier P. Sunscreen abuse for intentional sun exposure. Br J Dermatol. 2009;161 Suppl 3:40-5.

216. Ghiasvand R, Lund E, Edvardsen K, Weiderpass E, Veierød MB. Prevalence and trends of sunscreen use and sunburn among Norwegian women. Br J Dermatol. 2015;172(2):475-83.

217. Sinclair C, Foley P. Skin cancer prevention in Australia. Br J Dermatol. 2009;161 Suppl 3:116-23.

218. Bränström R, Ullén H, Brandberg Y. Attitudes, subjective norms and perception of behavioural control as predictors of sun-related behaviour in Swedish adults. Prev Med. 2004;39(5):992-9.

219. White KM, Starfelt LC, Young RM, Hawkes AL, Cleary C, Leske S, et al. A randomised controlled trial of an online theorybased intervention to improve adult Australians' sun-protective behaviours. Prev Med. 2015;72:19-22.

220. Hamilton K, Cleary C, White KM, Hawkes AL. Keeping kids sun safe: exploring parents' beliefs about their young child's sun-protective behaviours. Psychooncology. 2016;25(2):158-63.

221. Hunter S, Love-Jackson K, Abdulla R, Zhu W, Lee JH, Wells KJ, et al. Sun protection at elementary schools: a cluster randomized trial. J Natl Cancer Inst. 2010;102(7):484-92.

222. Gritz ER, Tripp MK, James AS, Carvajal SC, Harrist RB, Mueller NH, et al. An intervention for parents to promote preschool children's sun protection: effects of Sun Protection is Fun! Prev Med. 2005;41(2):357-66.

223. Sandhu PK, Elder R, Patel M, Saraiya M, Holman DM, Perna F, et al. Community-wide Interventions to Prevent Skin Cancer: Two Community Guide Systematic Reviews. Am J Prev Med. 2016;51(4):531-9.

224. CCMO. Central Committee on Research involving Human Subjects memorandum Behavioural research [Internet]. The Hague, NL: CCMO; 2002 [cited1Nov2020]. Available from:file:///D:/Libraries/Downloads/CCMO+note+Behavioural+research pdf.

225. Crutzen R, Ygram Peters GJ, Mondschein C. Why and how we should care about the General Data Protection Regulation. Psychol Health. 2019;34(11):1347-57.

226. von Elm E, Altman DG, Egger M, Pocock SJ, Gøtzsche PC, Vandenbroucke JP. The Strengthening the Reporting of Observational Studies in Epidemiology (STROBE) statement: guidelines for reporting observational studies. Bull World Health Organ. 2007;85(11):867-72.

227. TNS-KANTAR. Privacy Policy Online Survey [Internet]. Amsterdam, NL: Kantar TNS Nederland; 2019 [cited 1 Nov 2020]. Available from: https:/www.tns-nipo.com/getmedia/e03d519b-007b-4e77-800a-f737c5eaaa3d/Privacy-Policy-OnlineSurvey-Kantar-TNS-Public-(English).pdf.

228. Statistics Netherlands. Standaardonderwijsindeling [Internet]. The Hague, NL: Centraal Bureau voor de Statistiek; 2016 [cited 1 May 2020]. Available from: https://www.cbs.nl/nl-nl/onze-diensten/methoden/classificaties/onderwijs-enberoepen/standaard-onderwijsindeling--soi--/standaard-onderwijsindeling-2016.

229. Nuffic. Education in the Netherlands [Internet]. The Hague, NL: Nuffic; 2020 [cited 1 May 2020]. Available from: https:// www.nuffic.nl/en/subjects/education-in-the-netherlands/.

230. IBM Corp. Released 2016. IBM SPSS Statistics for Windows, Version 24.0. Armonk, NY IBM Corp.

231. Crutzen R, Peters GY, Noijen J. Using Confidence Interval-Based Estimation of Relevance to Select Social-Cognitive Determinants for Behavior Change Interventions. Front Public Health. 2017;5:165.

232. Moinester M, Gottfried R. Sample size estimation for correlations with pre-specified confidence interval. Quant Method Psychol. 2014;10(2):124-30.

233. Peters G-JY, Crutzen R. Knowing how effective an intervention, treatment, or manipulation is and increasing replication rates: accuracy in parameter estimation as a partial solution to the replication crisis. Psychol Health. 2020:1-19. 
234. R Core Team. 2019. R: A language and environment for statistical computing. R Foundation for Statistical Computing Vienna, Austria.

235. Peters G-J, Peters MG-J. Package 'userfriendlyscience'. 2018. Vienna: R Foundation for Statistical Computing. Available from: http://bioconductor.statistik.tu-dortmund.de/cran/web/packages/userfriendlyscience/userfriendlyscience.pdf.

236. Webb TL, Sheeran P. Does changing behavioral intentions engender behavior change? A meta-analysis of the experimental evidence. Psychol Bull. 2006;132(2):249-68.

237. Hamilton K, van Dongen A, Hagger MS. An extended theory of planned behavior for parent-for-child health behaviors: A meta-analysis. Health Psychol. 2020;39(10):863.

238. Sniehotta FF, Scholz U, Schwarzer R. Bridging the intention-behaviour gap: Planning, self-efficacy, and action control in the adoption and maintenance of physical exercise. Psychol Health. 2005;20(2):143-60.

239. van Osch L, Reubsaet A, Lechner L, de Vries $H$. The formation of specific action plans can enhance sun protection behavior in motivated parents. Prev Med. 2008;47(1):127-32.

240. Bailey RR. Goal Setting and Action Planning for Health Behavior Change. Am J Lifestyle Med. 2019;13(6):615-8.

241. Schwarzer R. Modeling health behavior change: How to predict and modify the adoption and maintenance of health behaviors. Appl Psychol. 2008;57(1):1-29.

242. Neal DT, Wood W, Quinn JM. Habits - A repeat performance. Curr Directions Psychol Sci. 2006;15(4):198-202.

243. Aarts H, Verplanken B, Van Knippenberg A. Predicting behavior from actions in the past: Repeated decision making or a matter of habit? J Appl Soc Psychol. 1998;28(15):1355-74.

244. Orbell S, Verplanken B. The automatic component of habit in health behavior: habit as cue-contingent automaticity. Health Psychol. 2010;29(4):374-83.

245. Schüz N, Eid M. Beyond the usual suspects: target group-and behavior-specific factors add to a theory-based sun protection intervention for teenagers. J Beh Med. 2013;36(5):508-19.

246. Pavey JL, Sparks P. Autonomy and reactions to health-risk information. Psychol Health. 2010;25(7):855-72.

247. Dixon H, Borland R, Hill D. Sun protection and sunburn in primary school children: the influence of age, gender, and coloring. Prev Med. 1999;28(2):119-30.

248. Glanz K, McCarty F, Nehl EJ, O'Riordan DL, Gies P, Bundy L, et al. Validity of self-reported sunscreen use by parents, children, and lifeguards. Am J Prev Med. 2009;36(1):63-9.

249. O'Riordan DL, Glanz K, Gies P, Elliott T. A pilot study of the validity of self-reported ultraviolet radiation exposure and sun protection practices among lifeguards, parents and children. Photochem Photobiol. 2008;84(3):774-8.

250. Diepgen TL, Mahler V. The epidemiology of skin cancer. Br J Dermatol. 2002;146 Suppl 61:1-6.

251. Garbe C, Leiter U. Melanoma epidemiology and trends. Clin Dermatol. 2009;27(1):3-9.

252. Argenziano G, Lallas A, Longo C, Moscarella E, Kyrgidis A, Ferrara G. Cutaneous Melanoma: A Pocket Guide for Diagnosis and Management. Cambridge MA, US: Academic Press; 2017.

253. Thomas NE, Edmiston SN, Alexander A, Millikan RC, Groben PA, Hao H, et al. Number of nevi and early-life ambient UV exposure are associated with BRAF-mutant melanoma. Cancer Epidemiol Biomarkers Prev. 2007;16(5):991-7.

254. Osterlind A, Tucker MA, Stone BJ, Jensen OM. The Danish case-control study of cutaneous malignant melanoma. II. Importance of UV-light exposure. Int J Cancer. 1988;42(3):319-24.

255. Godar DE. UV doses worldwide. Photochem Photobiol. 2005;81(4):736-49.

256. Wesson KM, Silverberg NB. Sun protection education in the United States: what we know and what needs to be taught Cutis. 2003;71(1):71-4, 7.

257. Geller AC, Colditz G, Oliveria S, Emmons K, Jorgensen C, Aweh GN, et al. Use of sunscreen, sunburning rates, and tanning bed use among more than 10000 US children and adolescents. Pediatrics. 2002;109(6):1009-14.

258. Harrison SL, MacLennan R, Buettner PG. Sun exposure and the incidence of melanocytic nevi in young Australian children. Cancer Epidemiol Biomarkers Prev. 2008;17(9):2318-24.

259. Coogan PF, Geller A, Adams M, Benjes LS, Koh HK. Sun protection practices in preadolescents and adolescents: a school-based survey of almost 25,000 Connecticut schoolchildren. J Am Acad Dermatol. 2001;44(3):512-9.

260. Antonov D, Hollunder M, Schliemann S, Elsner P. Ultraviolet Exposure and Protection Behavior in the General Population: A Structured Interview Survey. Dermatol. 2016;232(1):11-6.

261. Dodd AT, Morelli J, Mokrohisky ST, Asdigian N, Byers TE, Crane LA. Melanocytic nevi and sun exposure in a cohort of colorado children: anatomic distribution and site-specific sunburn. Cancer Epidemiol Biomarkers Prev. 2007;16(10):2136-43. 262. Eccles JS. The development of children ages 6 to 14. Future Child. 1999;9(2):30-44.

263. Bennetts K, Borlands R, Swerissen H. Sun protection behaviour of children and their parents at the beach. Psychol Health. 1991;5(4):279-87.

264. Fisher K, Lowe JB, Gillespie A, Balanda K, Baade P, Stanton W. The relationship between Australian Students' perceptions of parental behavior, school policies, and sun protection behaviors. J Health Educ. 1996;27(4):242-7.

265. Bush PJ, lannotti RJ. A Children's Health Belief Model. Med Care. 1990;28(1):69-86.

266. Hewitt AM, Stephens C. Healthy eating among 10 - 13-year-old New Zealand children: understanding choice using the Theory of Planned Behaviour and the role of parental influence. Psychol Health Med. 2007;12(5):526-35.

267. Sandström A, Cressey J, Stecksén-Blicks C. Tooth-brushing behaviour in 6-12 year olds. Int J Paediatr Dent. 2011;21(1):439.

268. Unkel JH, Fenton SJ, Hobbs G, Jr., Frere CL. Toothbrushing ability is related to age in children. ASDC J Dent Child. 1995;62(5):346-8.

269. Currie C, Nic Gabhainn S, Godeau E. The Health Behaviour in School-aged Children: WHO Collaborative Cross-National (HBSC) study: origins, concept, history and development 1982-2008. Int J Public Health. 2009;54 Suppl 2:131-9.

270. Telama R. Tracking of physical activity from childhood to adulthood: a review. Obes Facts. 2009;2(3):187-95.

271. Best JA, Thomson SJ, Santi SM, Smith EA, Brown KS. Preventing cigarette smoking among school children. Annu Rev 
Public Health. 1988;9:161-201.

272. Køster B, Søndergaard J, Nielsen JB, Allen M, Olsen A, Bentzen J. The validated sun exposure questionnaire: association of objective and subjective measures of sun exposure in a Danish population-based sample. Br J Dermatol. 2017;176(2):44656

273. Dennis LK, Kancherla V, Snetselaar LG. Adolescent attitudes towards tanning: does age matter? Ped Health 2009;3(6):565-78.

274. Bancroft J, Reinisch JM. Adolescence and puberty. Oxford, UK: Oxford University Press; 1990.

275. Rankin JL, Lane DJ, Gibbons FX, Gerrard M. Adolescent self-consciousness: Longitudinal age changes and gender differences in two cohorts. J Res Adolesc. 2004;14(1):1-21.

276. Patterson JM, McCubbin HI. Adolescent coping style and behaviors: conceptualization and measurement. J Adolesc. 1987;10(2):163-86.

277. Parcel GS, Meyer MP. Development of an instrument to measure children's health locus of control. Health Educ Monogr. 1978;6(2):149-59.

278. Cohen RY, Brownell KD, Felix MR. Age and sex differences in health habits and beliefs of schoolchildren. Health Psychol. 1990;9(2):208-24.

279. Glanz K, Mayer JA. Reducing ultraviolet radiation exposure to prevent skin cancer methodology and measurement. Am J Prev Med. 2005;29(2):131-42.

280. O'Riordan DL, Nehl E, Gies P, Bundy L, Burgess K, Davis E, et al. Validity of covering-up sun-protection habits: Association of observations and self-report J Am Acad Dermatol. 2009;60(5):739-44.

281. Pearson N, Timperio A, Salmon J, Crawford D, Biddle SJ. Family influences on children's physical activity and fruit and vegetable consumption. Int J Behav Nutr Phys Act. 2009;6:34.

282. Dennis LK, Vanbeek MJ, Beane Freeman LE, Smith BJ, Dawson DV, Coughlin JA. Sunburns and risk of cutaneous melanoma: does age matter? A comprehensive meta-analysis. . Ann Epidemiol. 2008;18(8):614-27.

283. Rodvall YE, Wahlgren C-F, Ullén HT, Wiklund KE. Factors related to being sunburnt in 7-year-old children in Sweden. Eur J Cancer. 2010;46(3):566-72.

284. Autier P, Doré JF, Cattaruzza MS, Renard F, Luther H, Gentiloni-Silverj F, et al. Sunscreen use, wearing clothes, and number of nevi in 6- to 7-year-old European children. European Organization for Research and Treatment of Cancer Melanoma Cooperative Group. J Natl Cancer Inst. 1998;90(24):1873-80.

285. Reinau D, Achermann C, Arnet N, Meier CR, Hatz C, Surber C. Sun protective behaviour of vacationers spending holidays in the tropics and subtropics. Br J Dermatol. 2014;171(4):868-74.

286. McLeod G, Insch A, Henry J. Reducing barriers to sun protection-Application of a holistic model for social marketing AMJ. 2011;19(3):212-22.

287. McLeod GFH, Reeder Al, Gray AR, McGee R. Unintended Sunburn: A Potential Target for Sun Protection Messages. J Skin Cancer. 2017;2017:6902942.

288. Køster B, Thorgaard C, Philip A, Clemmensen IH. Prevalence of sunburn and sun-related behaviour in the Danish population: a cross-sectional study. Scand J Public Health. 2010;38(5):548-52.

289. Li J, Uter W, Pfahlberg A, Gefeller O. A comparison of patterns of sun protection during beach holidays and everyday outdoor activities in a population sample of young German children. Br J Dermatol. 2012;166(4):803-10.

290. Duarte AF, Picoto A, Pereira ADC, Correia O. Sun protection in children: a behavioural study. Eur J Dermatol. 2018;28(3):338-42.

291. Kongsted A, Nielsen AM. Latent Class Analysis in health research. J Physiother. 2017;63(1):55-8.

292. Ministry of the Interior and Kingdom Relations. Provincies [Internet]. The Hague, NL: Ministry of Interior and Kingdom relations; 2020 [cited $1 \mathrm{Jul} 2020$ ]. Available from: https://www.regioatlas.nl/indelingen/indelingen_indeling/t/provincies.

293. Lanza ST, Rhoades BL. Latent class analysis: an alternative perspective on subgroup analysis in prevention and treatment. Prev Sci. 2013;14(2):157-68.

294. Schreiber JB. Latent class analysis: an example for reporting results. Res Social Adm Pharm. 2017;13(6):1196-201.

295. SAS Institute Inc. SAS version 9.4. Carry, NC, USA.

296. Thoonen, K., van Osch, L., Drittij, R., de Vries, H., \& Schneider, F. (2021). A qualitative exploration of parental perceptions regarding their children's sun exposure, sun protection and sunburns. Front Public Health, 9:596253.

297. de Maleissye MF, Beauchet A, Saiag P, Corrêa M, Godin-Beeckmann S, Haeffelin M, et al. Sunscreen use and melanocytic nevi in children: a systematic review. Pediatr Dermatol. 2013;30(1):51-9.

298. Gilaberte Y, Carrascosa JM. Sun Protection in Children: Realities and Challenges. Actas Dermosifiliogr (English ed). 2014;105(3):253-62.

299. Schlarbaum JP, Lazovich D, Dodd E, Hanson B, Polcari IC. Examination of use and barriers for five sun protection strategies in parents and their children. Pediatr Dermatol. 2020;37(5):827-32.

300. Adams MA, Mayer JA, Bowen DJ, Ji M. Season of interview and self-report of summer sun protection behaviors. Cancer Causes Control. 2009;20(2):153-62.

301. Dobbinson SJ, Jamsen K, Dixon HG, Spittal MJ, Lagerlund M, Lipscomb JE, et al. Assessing population-wide behaviour change: concordance of 10-year trends in self-reported and observed sun protection. Int J Public Health. 2014;59(1):157-66. 302. Hubbard G, Cherrie J, Gray J, Kyle RG, Nioi A, Wendelboe-Nelson C, et al. Sun protection education for adolescents: a feasibility study of a wait-list controlled trial of an intervention involving a presentation, action planning, and SMS messages and using objective measurement of sun exposure. BMC Public Health. 2020;20(1):131.

303. Kennedy C, Bajdik CD, Willemze R, De Gruijl FR, Bouwes Bavinck JN. The influence of painful sunburns and lifetime sun exposure on the risk of actinic keratoses, seborrheic warts, melanocytic nevi, atypical nevi, and skin cancer. $J$ Invest Dermatol. 2003;120(6):1087-93.

304. Glanz K, Gies P, O'Riordan DL, Elliott T, Nehl E, McCarty F, et al. Validity of self-reported solar UVR exposure compared 
with objectively measured UVR exposure. Cancer Epidemiol Biomarkers Prev. 2010;19(12):3005-12.

305. Christian H, Lester L, Trost SG, Trapp G, Schipperijn J, Boruff B, et al. Shade coverage, ultraviolet radiation and children's physical activity in early childhood education and care. Int J Public Health. 2019;64(9):1325-33.

306. Tripp MK, Peterson SK, Prokhorov AV, Shete SS, Lee JE, Gershenwald JE, et al. Correlates of Sun Protection and Sunburn in Children of Melanoma Survivors. Am J Prev Med. 2016;51(3):e77-85.

307. Ferlay J, Soerjomataram I, Dikshit R, Eser S, Mathers C, Rebelo M, et al. Cancer incidence and mortality worldwide: sources, methods and major patterns in GLOBOCAN 2012. Int J Cancer. 2015;136(5):E359-86.

308. Hoel DG, Berwick M, de Gruijl FR, Holick MF. The risks and benefits of sun exposure 2016. Dermatoendocrinol. 2016;8(1):e1248325.

309. Kulichová D, Dáňová J, Kunte C, Ruzicka T, Celko AM. Risk factors for malignant melanoma and preventive methods. Cutis. 2014;94(5):241-8.

310. Cust AE, Jenkins MA, Goumas C, Armstrong BK, Schmid H, Aitken JF, et al. Early-life sun exposure and risk of melanoma before age 40 years. Cancer Causes Control. 2011;22(6):885-97.

311. Jacovides C, Boland J, Rizou D, Kaltsounides N, Theoharatos G. School Students participation in monitoring solar radiation components: Preliminary results for UVB and UVA solar radiant fluxes. Renew. 2012;39(1):367-74.

312. McKenzie RL, Liley JB, Björn LO. UV radiation: balancing risks and benefits. Photochem Photobiol. 2009;85(1):88-98.

313. Bodekær M, Petersen B, Philipsen PA, Heydenreich J, Thieden E, Wulf HC. Sun exposure patterns of urban, suburban, and rural children: a dosimetry and diary study of 150 children. Photochem Photobiol Sci. 2015;14(7):1282-9.

314. Wiecker TS, Luther H, Buettner P, Bauer J, Garbe C. Moderate sun exposure and nevus counts in parents are associated with development of melanocytic nevi in childhood: a risk factor study in 1,812 kindergarten children. Cancer. 2003;97(3):62838.

315. McFarland AL, Zajicek JM, Waliczek TM. The relationship between parental attitudes toward nature and the amount of time children spend in outdoor recreation. J Leis Res. 2014;46(5):525-39.

316. Veitch J, Bagley S, Ball K, Salmon J. Where do children usually play? A qualitative study of parents' perceptions of influences on children's active free-play. Health Place. 2006;12(4):383-93.

317. Larson LR, Whiting JW, Green GT. Young people's outdoor recreation and state park use: Perceived benefits from the parent/guardian perspective. Child Youth Environ. 2013;23(3):89-118.

318. Miller KA, Langholz BM, Ly T, Harris SC, Richardson JL, Peng DH, et al. SunSmart: evaluation of a pilot school-based sun protection intervention in Hispanic early adolescents. Health Educ Res. 2015;30(3):371-9.

319. White KM, Zhao X, Starfelt Sutton LC, Young RM, Hamilton K, Hawkes AL, et al. Effectiveness of a theory-based sun-safe randomised behavioural change trial among Australian adolescents. Psychooncology. 2019;28(3):505-10.

320. Kelder SH, Perry CL, Klepp KI, Lytle LL. Longitudinal tracking of adolescent smoking, physical activity, and food choice behaviors. Am J Public Health. 1994;84(7):1121-6.

321. Liberati A, Altman DG, Tetzlaff J, Mulrow C, Gøtzsche PC, loannidis JP, et al. The PRISMA statement for reporting systematic reviews and meta-analyses of studies that evaluate health care interventions: explanation and elaboration. PLoS Med. 2009;6(7):e1000100.

322. Higgins JPT, Green S. Cochrane Handbook for Systematic Reviews of Interventions Version 5.1.0 [Internet]. London, UK: The Cochrane Collaboration; 2011. Available from: https://handbook-5-1.cochrane.org/.

323. Bramer WM, Giustini D, de Jonge GB, Holland L, Bekhuis T. De-duplication of database search results for systematic reviews in EndNote. J Med Libr Assoc. 2016;104(3):240-3.

324. Zaza S, Wright-De Agüero LK, Briss PA, Truman BI, Hopkins DP, Hennessy MH, et al. Data collection instrument and procedure for systematic reviews in the Guide to Community Preventive Services. Task Force on Community Preventive Services. Am J Prev Med. 2000;18(1 Suppl):44-74.

325. Glanz K, Lew RA, Song V, Murakami-Akatsuka L. Skin cancer prevention in outdoor recreation settings: effects of the Hawaii SunSmart Program. Eff Clin Pract. 2000;3(2):53-61.

326. Jackson N, Waters E. Criteria for the systematic review of health promotion and public health interventions. Health Promot Int. 2005;20(4):367-74.

327. Deeks JJ, Dinnes J, D'Amico R, Sowden AJ, Sakarovitch C, Song F, et al. Evaluating non-randomised intervention studies. Health Technol Assess. 2003;7(27): iii-x, 1-173.

328. Thomas $\mathrm{BH}$, Ciliska D, Dobbins $\mathrm{M}$, Micucci S. A process for systematically reviewing the literature: providing the research evidence for public health nursing interventions. Worldviews Evid Based Nurs. 2004;1(3):176-84.

329. Buller DB, Dobbinson S, English DR, Wakefield M, Buller MK. Rationale, design, and baseline data of a cross-national randomized trial on the effect of built shade in public parks for sun protection. Contemp Clin Trials. 2017;55:47-55.

330. Dobbinson S, Veitch J, Salmon J, Wakefield M, Staiger P, Maclnnis R, et al. Study protocol for a natural experiment in a lower socioeconomic area to examine the health-related effects of refurbishment to parks including built-shade (ShadePlus). BMJ Open. 2017;7(2):e013493.

331. Harrison SL, Buettner PG, Maclennan R. The North Queensland "Sun-Safe Clothing" study: design and baseline results of a randomized trial to determine the effectiveness of sun-protective clothing in preventing melanocytic nevi. Am $J$ Epidemiol. 2005;161(6):536-45.

332. Harrison SL. Sun-Safe Clothing Helps to Prevent the Development of Pigmented Moles. Results of a Randomised Control Trial in Young Australian Children. In Proceedings of the 100 Years of Tropical Medicine Conference 11th, Townsville, Australia; 11 October 2013. [cited 4 March 2019]. Available from: https://www.tropmed.org/wpcontent/uploads/2015/06/ annalsV14N1.pdf.

333. Swinburn B, Egger G, Raza F. Dissecting obesogenic environments: the development and application of a framework for identifying and prioritizing environmental interventions for obesity. Prev Med. 1999;29(6 Pt 1):563-70.

334. Gallagher RP, Rivers JK, Lee TK, Bajdik CD, McLean DI, Coldman AJ. Broad-spectrum sunscreen use and the 
development of new nevi in white children: A randomized controlled trial. JAMA. 2000;283(22):2955-60.

335. Bauer J, Büttner P, Wiecker TS, Luther H, Garbe C. Interventional study in 1,232 young German children to prevent the development of melanocytic nevi failed to change sun exposure and sun protective behavior. Int J Cancer. 2005;116(5):75561.

336. Barankin B, Liu K, Howard J, Guenther L. Effects of a sun protection program targeting elementary school children and their parents. J Cutan Med Surg. 2001;5(1):2-7.

337. Dobbinson SJ, White V, Wakefield MA, Jamsen KM, White V, Livingston PM, et al. Adolescents' use of purpose built shade in secondary schools: cluster randomised controlled trial. BMJ. 2009;338:b95.

338. Dobbinson SJ; Simmons J, Chamberlain J, Salmon J, Wakefield M, Staiger P, Maclnnis R Veitch J. Creating Activity Friendly Parks with Shade: The ShadePlus Intervention. In Proceedings of the Public Health Prevention Conference 12th, Melbourne, Australia; 12-14 June 2019. [cited 4 March 2019]. Available from: https://docs.wixstatic.com/ugd/ a52314_247935f69fb44088baeccd3a5c9b45dc.pdf.

339. Buller DB, English DR, Buller MK, Simmons J, Chamberlain JA, Wakefield M, et al. Shade Sails and Passive Recreation in Public Parks of Melbourne and Denver: A Randomized Intervention. Am J Public Health. 2017;107(12):1869-75.

340. British Association of Dermatologists. Sunscreen and Sun Safety Factsheet [Internet]. London, UK: British Association of Dermatologists; 2013. [cited 13 Feb 2019]. Available from: http://www.bad.org.uk/shared/get-file. ashx?id=3917\& itemtype=document.

341. Das RB. UV radiation protective clothing. Open Text J. 2010;3(1).

342. Leger LS, Kolbe L, Lee A, McCall DS, Young IM. School health promotion. Global perspectives on health promotion effectiveness: Springer. 2007. p. 107-24.

343. Schools for Health in Europe. State of the Art: Health Promoting Schools in Europe [Internet]. 2013 [cited 23 Sept 2019]. Available from: https://www.radix.ch/files/508NYKY/she_factsheet_1_2013.pdf.

344. Langford R, Bonell CP, Jones HE, Pouliou T, Murphy SM, Waters E, et al. The WHO Health Promoting School framework for improving the health and well-being of students and their academic achievement. Cochrane Database Syst Rev. 2014(4). 345. Langford R, Bonell C, Jones H, Pouliou T, Murphy S, Waters E, et al. The World Health Organization's Health Promoting Schools framework: a Cochrane systematic review and meta-analysis. BMC Public Health. 2015;15(1):130.

346. Townsend JS, Pinkerton B, McKenna SA, Higgins SM, Tai E, Steele CB, et al. Targeting children through school-based education and policy strategies: comprehensive cancer control activities in melanoma prevention. J Am Acad Dermatol. 2011;65(5 Suppl 1):S104-13.

347. Bronfenbrenner U. Ecological systems theory. In: Vasta R, editors. Six theories of child development: Revised formulations and current issues. London, UK: Jessica Kingsley Publishers; 1992. p. 187-249.

348. Diffey BL, Norridge Z. Reported sun exposure, attitudes to sun protection and perceptions of skin cancer risk: a survey of visitors to Cancer Research UK's SunSmart campaign website. The Br J Dermatol. 2009;160(6):1292-8.

349. Haluza D, Simic S, Moshammer H. Sun Exposure Prevalence and Associated Skin Health Habits: Results from the Austrian Population-Based UVSkinRisk Survey. Int J Environ Res Public Health. 2016;13(1).

350. Heerfordt IM, Philipsen PA, Wulf HC. Sun behaviour on the beach monitored by webcam photos. Public Health 2018;155:88-90.

351. Barrett F, Usher K, Woods C, Conway J. Sun protective behaviours during maximum exposure to ultraviolet radiation when undertaking outdoor activities: an integrated literature review. J Public Health. 2019;27(3):393-405.

352. Walkosz BJ, Scott MD, Buller DB, Andersen PA, Beck L, Cutter GR. Prevalence of Sun Protection at Outdoor Recreation and Leisure Venues at Resorts in North America. Am J Health Educ. 2017;48(2):90-9.

353. Wright CY, Reeder Al. Youth solar ultraviolet radiation exposure, concurrent activities and sun-protective practices: a review. Photochem Photobiol. 2005;81(6):1331-42.

354. King L, Xiang F, Swaminathan A, Lucas RM. Measuring sun exposure in epidemiological studies: Matching the method to the research question. Photochem Photobiol. 2015;153:373-9.

355. Sallis JF, McKenzie TL, Conway TL, Elder JP, Prochaska JJ, Brown M, et al. Environmental interventions for eating and physical activity: a randomized controlled trial in middle schools. Am J Prev Med. 2003;24(3):209-17.

356. Brug J, van Lenthe $F$, editors. Environmental determinants and interventions for physical activity, nutrition and smoking: a review [Internet]. Zoetermeer, NL: Speed-print; 2005. Available from: https://www.researchgate.net/profile/ Johannes_Brug/publication/268032185_Environmental_determinants_and_interventions_for_physical_activity_nutrition_ and_smoking_A_Review/links/5641c8c308ae24cd3e427577/Environmental-determinants-and-interventions-for-physicalactivity-nutrition-and-smoking-A-Review.pdf.

357. Glanz K, Saraiya M. Using evidence-based community and behavioral interventions to prevent skin cancer: opportunities and challenges for public health practice. Prev Chronic Dis. 2005;2(2):A03.

358. Victora CG, Habicht JP, Bryce J. Evidence-based public health: moving beyond randomized trials. Am J Public Health 2004; $94(3): 400-5$.

359. Charrois TL. Systematic reviews: what do you need to know to get started? Can J Hosp Pharm. 2015;68(2):144-8.

360. Paez A. Gray literature: An important resource in systematic reviews. J Evid Based Med. 2017;10(3):233-40.

361. Scherer RW, Saldanha IJ. How should systematic reviewers handle conference abstracts? A view from the trenches Syst Rev. 2019;8(1):264.

362. Moher D, Liberati A, Tetzlaff J, Altman DG. Preferred reporting items for systematic reviews and meta-analyses: the PRISMA statement. PLoS Med. 2009;6(7):e1000097.

363. Khan KS, Kunz R, Kleijnen J, Antes G. Five steps to conducting a systematic review. J R Soc Med. 2003;96(3):118-21.

364. Armijo-Olivo S, Stiles CR, Hagen NA, Biondo PD, Cummings GG. Assessment of study quality for systematic reviews: a comparison of the Cochrane Collaboration Risk of Bias Tool and the Effective Public Health Practice Project Quality Assessment Tool: methodological research. J Eval Clin Pract. 2012;18(1):12-8. 
365. Mercer SL, Green LW, Rosenthal AC, Husten CG, Khan LK, Dietz WH. Possible lessons from the tobacco experience for obesity control. Am J Clin Nutr. 2003;77(4 Suppl):1073s-82s.

366. Davison KK, Lawson CT. Do attributes in the physical environment influence children's physical activity? A review of the literature. Int J Behav Nutr Phys Act. 2006;3:19.

367. de Vries SI, Bakker I, van Mechelen W, Hopman-Rock M. Determinants of activity-friendly neighborhoods for children: results from the SPACE study. Am J Health Promot. 2007;21(4 Suppl):312-6.

368. Ferreira I, van der Horst K, Wendel-Vos W, Kremers S, van Lenthe FJ, Brug J. Environmental correlates of physical activity in youth - a review and update. Obes Rev. 2007;8(2):129-54.

369. Godar DE. Worldwide increasing incidences of cutaneous malignant melanoma. J Skin Cancer. 2011;2011:858425.

370. Greinert R, de Vries E, Erdmann F, Espina C, Auvinen A, Kesminiene A, et al. European Code against Cancer 4th Edition: Ultraviolet radiation and cancer. Cancer Epidemiol. 2015;39 Suppl 1:S75-83.

371. Dobbinson S, Niven P, Buller D, Allen M, Gies P, Warne C. Comparing Handheld Meters and Electronic Dosimeters for Measuring Ultraviolet Levels under Shade and in the Sun. Photochem Photobiol. 2016;92(1):208-14.

372. Hill D, White V, Marks R, Theobald T, Borland R, Roy C. Melanoma prevention: behavioral and nonbehavioral factors in sunburn among an Australian urban population. Prev Med. 1992;21(5):654-69.

373. Boyett T, Davy L, Weathers L, Campbell R, Van Durme D, Roetzheim RG. Sun protection of children at the beach. J Am Board Fam Med. 2002;15(2):112-7.

374. Gage R, Barr M, Stanley J, Reeder A, Mackay C, Smith M, et al. Sun protection and shade availability in New Zealand's outdoor recreation spaces. NZ Med J. 2018;131:30-7.

375. Lovato C, Shoveller J, Mills C, Gallagher R, Rivers J, Campbell S. Canadian national workshop on measurement of sunrelated behaviours. Chronic Dis Inj Canada. 1999;20(2):96.

376. McGee R, Williams S, Glasgow H. Sunburn and sun protection among young children. J Paediatr Child Health. 1997;33(3):234-7.

377. Cokkinides V, Weinstock M, Glanz K, Albano J, Ward E, Thun M. Trends in sunburns, sun protection practices, and attitudes toward sun exposure protection and tanning among US adolescents, 1998-2004. Pediatrics. 2006;118(3):853-64.

378. Skinner CS, Tiro J, Champion VL. Background on the health belief model. In: Glanz K, Rimer BK, Viswanath K, editors Health behavior: Theory, research, and practice, 5th ed. San Francisco, CA, US: Jossey-Bass; 2015. Chapter 5, p. 75-94.

379. Kasparian NA, McLoone JK, Meiser B. Skin cancer-related prevention and screening behaviors: a review of the literature. J Behav Med. 2009;32(5):406-28.

380. Glanz K, Lew RA, Song V, Cook VA. Factors associated with skin cancer prevention practices in a multiethnic population. Health Educ Behav. 1999;26(3):344-59.

381. Armstrong BK, Cust AE. Sun exposure and skin cancer, and the puzzle of cutaneous melanoma: A perspective on Fears et al. Mathematical models of age and ultraviolet effects on the incidence of skin cancer among whites in the United States. American Journal of Epidemiology 1977; 105: 420-427. Cancer Epidem. 2017;48:147-56.

382. Battie C, Gohara M, Verschoore M, Roberts W. Skin cancer in skin of color: an update on current facts, trends, and misconceptions. J Drugs Dermatol. 2013;12(2):194-8.

383. Stinco G, Favot F, Quinkenstein E, Zanchi M, Valent F, Patrone P. Children and sun exposure in the northeast of Italy. Pediatr Dermatol. 2005;22(6):520-4.

384. Jürges $H$, Reinhold S, Salm M. Does schooling affect health behavior? Evidence from the educational expansion in Western Germany. Econom Educ Rev. 2011;30(5):862-72.

385. Berrigan D, Dodd K, Troiano RP, Krebs-Smith SM, Barbash RB. Patterns of health behavior in U.S. adults. Prev Med. 2003;36(5):615-23.

386. Moore GF, Littlecott HJ. School- and family-level socioeconomic status and health behaviors: multilevel analysis of a national survey in wales, United Kingdom. J Sch Health. 2015;85(4):267-75.

387. Marmot M, Allen J, Bell R, Bloomer E, Goldblatt P. WHO European review of social determinants of health and the health divide. Lancet. 2012;380(9846):1011-29.

388. Abeck D, Feucht J, Schäfer T, Behrendt H, Krämer U, Ring J. Parental sun protection management in preschool children Photodermatol Photoimmunol Photomed. 2000;16(3):139-43.

389. Scerri L, Aquilina S, Gauci AA, Dalmas M. Sun awareness and sun protection practices in Malta. J Eur Acad Dermatol Venereol. 2002;16(1):47-52.

390. Cardinez CJ, Cokkinides VE, Weinstock MA, O'Connell MC. Sun protective behaviors and sunburn experiences in parents of youth ages 11 to 18. Prev Med. 2005;41(1):108-17.

391. Donavan DT, Singh SN. Sun-safety behavior among elementary school children: the role of knowledge, social norms, and parental involvement. Psychol Rep. 1999;84(3):831-6.

392. Brown KA, Ogden J, Vögele C, Gibson EL. The role of parental control practices in explaining children's diet and BMI. Appetite. 2008;50(2-3):252-9.

393. Gubbels JS, Gerards SM, Kremers SP. The association of parenting practices with toddlers' dietary intake and BMI, and the moderating role of general parenting and child temperament. Public Health Nutr. 2020;23(14):2521-9.

394. Költő A, Gavin A, Kelly C, Gabhainn SN. Sun and ultraviolet light-related behaviours in schoolchildren of Ireland [Internet]. 2020; Available from: http://www.nuigalway.ie/media/healthpromotionresearchcentre/hbscdocs/shortreports/2018-AKSUN-AND-UV-LIGHT-REPORT-19-March-2020.pdf

395. Rouhani P, Parmet Y, Bessell AG, Peay T, Weiss A, Kirsner RS. Knowledge, attitudes, and behaviors of elementary school students regarding sun exposure and skin cancer. Pediatr Dermatol. 2009;26(5):529-35.

396. Armitage CJ, Conner M. Efficacy of the Theory of Planned Behaviour: a meta-analytic review. Br J Soc Psychol. 2001;40(Pt 4):471-99.

397. Sheeran P, Webb TL. The intention-behavior gap. Soc Personal Psychol Compass. 2016;10(9):503-18. 
398. Craciun C, Schüz N, Lippke S, Schwarzer R. A mediator model of sunscreen use: a longitudinal analysis of socialcognitive predictors and mediators. Int J Behav Med. 2012;19(1):65-72.

399. Lescano CM, Rodrigue JR. Skin cancer prevention behaviors among parents of young children. Children's Health Care. 1997;26(2):107-14.

400. Maddock JE, Redding CA, Rossi JS, Weinstock MA. Development and validation of an appearance motivation attitudes scale for sun protection. Psychol Health. 2005;20(6):775-88.

401. Sutton S. How does the health action process approach (HAPA) bridge the intention-behavior gap? An examination of the model's causal structure. Appl Psychol. 2008;57(1):66-74.

402. Sniehotta FF, Scholz U, Schwarzer R. Action plans and coping plans for physical exercise: A longitudinal intervention study in cardiac rehabilitation. Br J Health Psychol. 2006;11(Pt 1):23-37.

403. Godinho CA, Alvarez MJ, Lima ML, Schwarzer R. Will is not enough: coping planning and action control as mediators in the prediction of fruit and vegetable intake. $\mathrm{Br} J$ Health Psychol. 2014;19(4):856-70.

404. Kasten S, van Osch L, Candel M, de Vries $H$. The influence of pre-motivational factors on behavior via motivational factors: a test of the I-Change model. BMC Psychol. 2019;7(1):7.

405. Robinson JK, Rigel DS, Amonette RA. Trends in sun exposure knowledge, attitudes, and behaviors: 1986 to $1996 . \mathrm{J}$ Am Acad Dermatol. 1997;37(2 Pt 1):179-86.

406. Bränström R, Brandberg Y, Holm L, Sjöberg L, Ullén H. Beliefs, knowledge and attitudes as predictors of sunbathing habits and use of sun protection among Swedish adolescents. Eur J Cancer Prev. 2001;10(4):337-45.

407. Janssen $E$, van Osch L, Lechner $L$, Candel $M$, de Vries $H$. Thinking versus feeling: Differentiating between cognitive and affective components of perceived cancer risk. Psychol Health. 2012;27(7):767-83.

408. Lowe JB, McDermott LJ, Stanton WR, Clavarino A, Balanda KP, McWhirter B. Behavior of caregivers to protect their infants from exposure to the sun in Queensland, Australia. Health Educ Res. 2002;17(4):405-14.

409. Cafri G, Thompson JK, Roehrig M, van den Berg P, Jacobsen PB, Stark S. An investigation of appearance motives for tanning: The development and evaluation of the Physical Appearance Reasons For Tanning Scale (PARTS) and its relation to sunbathing and indoor tanning intentions. Body Image. 2006;3(3):199-209.

410. Gore SM, Altman DG. Statistics in practice. London, UK: British Medical Association; 1982. doi: 10.1002/sim.4780010416. 411. Weisberg HF. The methodological strengths and weaknesses of survey research. In: Donsbach W, Traugott MW. The SAGE handbook of public opinion research. London, UK: Sage Publications Ltd; 2008. p. 223-31.

412. Tripp MK, Vernon SW, Gritz ER, Diamond PM, Mullen PD. Children's skin cancer prevention: a systematic review of parents' psychosocial measures. Am Am J Prev Med. 2013;44(3):265-73.

413. Glanz K, Yaroch AL, Dancel M, Saraiya M, Crane LA, Buller DB, et al. Measures of sun exposure and sun protection practices for behavioral and epidemiologic research. Arch Dermatol. 2008;144(2):217-22.

414. Creech LL, Mayer JA. Ultraviolet radiation exposure in children: a review of measurement strategies. Ann Behav Med. 1997;19(4):399-407.

415. Buller DB, Reynolds KD, Buller MK, Massie K, Berteletti J, Ashley J, et al. Parent reports of sun safety communication and behaviour for students in a randomised trial on a school policy implementation intervention. Aust NZ J Public Health 2020;44(3):208-14.

416. Gooden WE, Struble KD. Perceived parental behavior and the social desirability response set. $J$ Youth Adolesc 1990;19(6):605-13.

417. Petersen B, Thieden E, Lerche CM, Wulf HC. Validation of self-reported erythema: comparison of self-reports, researcher assessment and objective measurements in sun worshippers and skiers. J Eur Acad Dermatol Venereol. 2013;27(2):214-9.

418. Levin KA. Study design III: Cross-sectional studies. Evid Based Dent. 2006;7(1):24-5.

419. Termorshuizen F, Wijga A, Garssen J, Den Outer PN, Slaper H, Van Loveren H. Exposure to solar ultraviolet radiation in young Dutch children: assessment by means of a 6 -week retrospective questionnaire. $J$ Expo Anal Environ Epidemiol. 2002;12(3):204-13.

420. Héry C, Tryggvadottir L, Sigurdsson T, Ólafsdóttir E, Sigurgeirsson B, Jonasson JG, et al. A melanoma epidemic in Iceland: possible influence of sunbed use. Am J Epidem. 2010;172(7):762-7.

421. Diffey B. The early days of personal solar ultraviolet dosimetry. Atmosphere. 2020;11(2):125.

422. Allen MW, Swift N, Nield KM, Liley B, McKenzie RL. Use of Electronic UV Dosimeters in Measuring Personal UV Exposures and Public Health Education. Atmosphere. 2020;11(7):744.

423. Buller DB, Berwick M, Lantz K, Buller MK, Shane J, Kane I, et al. Smartphone mobile application delivering personalized, real-time sun protection advice: a randomized clinical trial. JAMA Dermatol. 2015;151(5):497-504.

424. Buller DB, Berwick M, Shane J, Kane I, Lantz K, Buller MK. User-centered development of a smart phone mobile application delivering personalized real-time advice on sun protection. Transl Behav Med. 2013;3(3):326-34.

425. Vuong K, Armstrong BK, McGeechan K, Cust AE. Personalized melanoma risk assessments and tailored prevention advice: a pragmatic randomized controlled trial in Australian general practice. Fam Pract. 2019;36(2):237-46.

426. Qian C, Yuan C, Tan Y, Liu X, Dong Y, Yang L, et al. Comparing performance of C hromameter ${ }^{\circledR}, M_{\text {exameter }}{ }^{\circledR}$ and fullfield laser perfusion imaging for measurement of ultraviolet B light-induced erythema. Clin Exp Dermatol. 2015;40(4):43840.

427. Armitage CJ. Evidence that implementation intentions reduce dietary fat intake: a randomized trial. Health Psychol. 2004;23(3):319-23.

428. van Bree RJ, van Stralen MM, Mudde AN, Bolman C, de Vries H, Lechner L. Habit as mediator of the relationship between prior and later physical activity: A longitudinal study in older adults. Psychol Sport Exercise. 2015;19:95-102.

429. Hamilton K, Gibbs I, Keech JJ, Hagger MS. Reasoned and implicit processes in heavy episodic drinking: An integrated dual-process model. Br J Health Psychol. 2020;25(1):189-209.

430. Hamilton K, van Dongen A, Hagger MS. Theory of planned behavior meta-analysis for parent-for-child behaviors 
[preprint]. 2020. Available from: https://psyarxiv.com/mv4fc/. doi: 10.31234/osf.io/mv4fc.

431. Srinivasan S, O'Fallon LR, Dearry A. Creating healthy communities, healthy homes, healthy people: initiating a research agenda on the built environment and public health. Am J Public Health. 2003;93(9):1446-50.

432. Gritz ER, Tripp MK, Peterson SK, Prokhorov AV, Shete SS, Urbauer DL, et al. Randomized controlled trial of a sun protection intervention for children of melanoma survivors. Cancer Epidemiol Biomarkers Prev. 2013;22(10):1813-24.

433. Warner LM, Schüz B, Wolff JK, Parschau L, Wurm S, Schwarzer R. Sources of self-efficacy for physical activity. Health Psychol. 2014;33(11):1298-308.

434. Sümen A, Öncel S. The effect of 'I am Protecting my Child from the Sun'programme on parental sun protection behaviours: Randomized controlled trial. J Adv Nursing. 2021;77(1):387-400.

435. Heckman CJ, Manne SL, Kloss JD, Bass SB, Collins B, Lessin SR. Beliefs and intentions for skin protection and UV exposure in young adults. Am J Health Behav. 2011;35(6):699-711.

436. Thomas C, Peñas PF. Why do young adults tan? J Eur Acad Dermatol Venereol. 2017;31(5):e259.

437. Patel AR, Zaslow TL, Wren TA, Daoud AK, Campbell K, Nagle K, et al. A characterization of sun protection attitudes and behaviors among children and adolescents in the United States. Prev Med Rep. 2019;16:100988.

438. Cash TF, Smolak L. Understanding body images: Historical and contemporary perspectives. In: Cash TS, Smolak L, editors. Body image: A handbook of science, practice, and prevention. New York, NY, US: The Guilford Press; 2011. p. 3-11.

439. Marks R, Hill D. Behavioural change in adolescence: a major challenge for skin-cancer control in Australia. Med J Aust. 1988;149(10):514-5.

440. Perez D, Kite J, Dunlop SM, Cust AE, Goumas C, Cotter T, et al. Exposure to the 'Dark Side of Tanning' skin cancer prevention mass media campaign and its association with tanning attitudes in New South Wales, Australia. Health Educ Res. 2015;30(2):336-46.

441. Stapleton JL, Hillhouse J, Levonyan-Radloff K, Manne SL. Review of interventions to reduce ultraviolet tanning: Need for treatments targeting excessive tanning, an emerging addictive behavior. Psychol Addict Behav. 2017;31(8):962-78.

442. Stapleton J, Turrisi R, Hillhouse J, Robinson JK, Abar B. A comparison of the efficacy of an appearance-focused skin cancer intervention within indoor tanner subgroups identified by latent profile analysis. J Behav Med. 2010;33(3):181-90.

443. Sheeran P, Gollwitzer PM, Bargh JA. Nonconscious processes and health. Health Psychol. 2013;32(5):460-73.

444. Craciun C, Schüz N, Lippke S, Schwarzer R. Enhancing planning strategies for sunscreen use at different stages of change. Health Educ Res. 2012;27(5):857-67.

445. Wiedemann AU, Lippke S, Reuter T, Ziegelmann JP, Schüz B. The more the better? The number of plans predicts health behaviour change. Appl Psychol Health Well-Being. 2011;3(1):87-106.

446. Bélanger-Gravel A, Godin G, Amireault S. A meta-analytic review of the effect of implementation intentions on physical activity. Health Psychol Rev. 2013;7(1):23-54.

447. Kok G, Peters LWH, Ruiter RAC. Planning theory- and evidence-based behavior change interventions: a conceptual review of the intervention mapping protocol. Psicol Reflex Crit. 2017;30(1):19.

448. Kremers S, Sleddens E, Gerards S, Gubbels J, Rodenburg G, Gevers D, et al. General and food-specific parenting: measures and interplay. Child Obes. 2013;9 Suppl(Suppl 1):S22-31.

449. Gubbels JS, Stessen K, van de Kolk I, de Vries NK, Thijs C, Kremers SPJ. Energy balance-related parenting and childcare practices: The importance of meso-system consistency. PloS One. 2018;13(9):e0203689.

450. Wilson DK, Lawman HG. Health promotion in children and adolescents: An integration of the biopsychosocial model and ecological approaches to behavior change. In: Roberts MC, Steele RG, editors. Handbook of pediatric psychology, 4th ed. New York, NY, US: The Guilford Press; 2009. p. 603-617.

451. Geller AC, Dickerman BA, Taber JM, Dwyer LA, Hartman AM, Perna FM. Skin cancer interventions across the cancer control continuum: A review of experimental evidence (1/1/2000-6/30/2015) and future research directions. Prev Med. 2018;111:442-50.

452. National Institute for Public Health and the Environment. De Gezonde School [Internet]. De Bilt, NL: RIVM; 2019 [cited 1 Oct 2020]. Available from: https://www.rivm.nl/en/gezonde-school.

453. Bartelink NHM, van Assema P, Kremers SPJ, Savelberg H, Oosterhoff M, Willeboordse M, et al. Can the Healthy Primary School of the Future offer perspective in the ongoing obesity epidemic in young children? A Dutch quasi-experimental study. BMJ Open. 2019;9(10):e030676.

454. Cancer Council Australia. SunSmart national policy statement [Internet]. Australia: Cancer Council; 2019 [cited 20 Oct 2020]. Available from: https://www.cancer.org.au/about-us/policy-and-advocacy/position-statements/sunsmart.

455. Haynes A, Nathan A, Dixon H, Wakefield M, Dobbinson S. Sun-protective clothing and shade use in public outdoor leisure settings from 1992 to 2019: Results from cross-sectional observational surveys in Melbourne, Australia. Prev Med. 2020;139:106230.

456. Køster B, Meyer M, Andersson T, Engholm G, Dalum P. Development in sunburn 2007-2015 and skin cancer projections 2007-2040 of campaign results in the Danish population. Med. 2018;97(41):e12738.

457. Køster B, Meyer MKH, Søgaard J, Dalum P. Benefit-Cost Analysis of the Danish Sun Safety Campaign 2007-2015: Cost Savings from Sunburn and Sunbed Use Reduction and Derived Skin Cancer Reductions 2007-2040 in the Danish Population. Pharmacoecon Open. 2020;4(3):419-25.

458. Ministry of Infrastructure and Water Management. Uitvoeren met ambitie. Uitvoeringsprogramma 2018-2019. Nationale Klimaatadaptatiestrategie (NAS) [Internet]. The Hague, NL: Ministerie van Infrastructuur en Waterstaat; 2018. [cited 20 Oct 2020]. Available from: https://www.rijksoverheid.nl/documenten/rapporten/2018/04/04/uitvoeren-met-ambitieuitvoeringsprogramma-2018-2019-nationale-klimaatadaptatiestrategie-nas.

459. Huynen M van Vliet A, Staatsen B, Hall L, Zwartkruis J, Kruize H, et al. Kennisagenda Klimaat en Gezondheid [Internet]. The Hague, NL: ZonMw; 2019. [cited 20 Oct 2020]. Available from: https://www.zonmw.nl/fileadmin/zonmw/documenten/ Gezondheidsbescherming/Kennisagenda_Klimaat_en_Gezondheid_digi_versie.pdf. 
460. van Dijk A, Hagens W, Slaper H, Boekema M. UV-index action plan: Version 2019 [Internet]. De Bilt, NL: RIVM; 2019. [cited 20 Oct 2020]. Available from: https://www.rivm.nl/bibliotheek/rapporten/2019-0078.pdf.

461. Graffiti6 (2010). Stare Into The Sun (Lyrics) 
156 Breaking through the clouds 
Summary 
In the Netherlands, skin cancer - including non-melanoma and melanoma skin cancer - has increased dramatically since the late 1980 s and currently, rates are globally among the highest. Further, skin cancer puts a high and increasing burden on our society in terms of healthcare costs, with future prognoses for this burden being poor as well. The excessive skin cancer increase is mostly caused by an ageing population and changes in sun-seeking behaviours. Since skin cancer is regarded one of the most preventable malignancies, efforts for improving sun protection are warranted. Unprotected sun exposure and sunburn are crucial risk factors for developing skin cancer in life, especially during childhood. This makes sun protection in early life highly important. Since children are not (yet) able to perform sun protection behaviours themselves, parents play a crucial role in pursuing children's sun safety. Sun protection strategies consist of multiple, complex behaviours - e.g. sunscreen use, protective clothing and seeking shade, and are dependent of various factors, such as the UV-index, children's skin type, and duration of sun exposure. Currently, children's sun protection is suboptimal and efforts are warranted to improve their sun safety. Furthermore, in a Dutch context, little is known about children's sun safety. Hence, to understand children's sun safety, multiple behaviours need investigation, from both children's and parents' perspectives, in different settings.

\section{Chapter I}

In Chapter 1, a general introduction to the studies presented in this thesis is provided. This thesis was aimed at contributing to the current knowledge regarding sun safety of children (aged between 4 and 12 years old) in the Netherlands in order to provide directions for sun safety interventions. A comprehensive strategy was used to investigate children's sun safety, by examining multiple parental and children's protection behaviours in different sun exposure situations. The sun protection behaviours described throughout our studies are therefore divided in: 1) direct performance, by e.g. applying sunscreen, putting on sunglasses; and 2) indirect performance, by teaching children to perform sun protection behaviours themselves. Moreover, sun exposure situations are distinguished in: 1) planned sun exposure (i.e. when going to the beach or swimming pool); and 2) incidental sun exposure (when engaging in other activities, e.g. cycling or playing outside).

\section{Chapter 2}

Chapter 2 aimed to gain a broad insight into parental perceptions regarding children's sunburn, sun exposure and parental sun protection behaviours using a qualitative approach. Further, we proposed to understand situations in which children experience sunburn in the Netherlands. In total, 26 interviews were conducted ( $n=17$ mothers of $n=17$ girls). Execution, transcription, and coding of the interviews was done by two researchers, demonstrating an interrater reliability of $d=.84$. The findings indicated that sunburn was highly prevalent among children and that parents often underestimated their children's sunburn occurrence. Settings in which sunburn occurred most frequent were water-related with limited shade facilities. Interestingly, all parents reported at least one sun protection behaviour. Of those, sunscreen use was most often applied by parents. Knowledge deficits concerning the UV-index and correct sunscreen application were also detected, although all parents reported the use of sunscreen. Furthermore, parents often mentioned being unprepared for (a longer duration of) sun exposure and seemed to particularly underrate the need for sun protection strategies in incidental situations. Finally, a striking finding was that the majority of parents indicated to hold positive attitudes towards a tanned skin, which in some cases even intentionally resulted in less sun protection behaviours. Based on the findings in this study, we recommended to educate parents about correct sunscreen application and stimulate clothing and seeking shade behaviours as well, to shape a sun protection promoting physical environment by e.g. providing 


\section{Chapter 3}

In Chapter 3, a comprehensive investigation of relevant determinants in predicting both direct and indirect parental sun protection intentions and behaviours in different sun exposure settings, is described. We used longitudinal data from two measurements ( $\mathrm{n}=904$ at $\mathrm{T} 1 ; \mathrm{n}=670$ at T2) to conduct analyses in $R$, using the CIBER approach. Univariate distributions (sample means) and associations with behavioural outcomes (correlates), visualized the relevance of socio-cognitive determinants. In total, the relevance of 27 generic (e.g. knowledge, risk-perceptions) and behaviour-specific (e.g. attitudes, self-efficacy) determinants were examined. Results indicated that behaviour-specific determinants were most relevant in predicting parental sun protection intentions and behaviours, with attitude, self-efficacy and action planning explaining most of the variance. Moreover, parental self-efficacy regarding sun protection behaviours demonstrated substantial room for improvement. Furthermore, the results indicated that a positive attitude regarding a tanned skin, as well as children's previous sunburn, were negatively associated with parental sun protection behaviours. Additionally, the total explained variances of all determinants together demonstrated highest explained variance for shade-seeking - and lowest for indirect behaviours. We revealed comprehensive insight in important socio-cognitive determinants related to parental sun protection behaviours and provided guidance for composing future educational sun safety interventions.

\section{Chapter 4}

Chapter 4 describes findings from a study in which we examined reported parental and children's own sun protection behaviours. We were particularly interested in testing whether a behavioural shift - in which children performed sun protection behaviours more often than their parents did for them - would be present. We used cross-sectional data from our longitudinal survey study ( $n=1053$ at T1) and conducted two-way ANOVA and linear regression analyses in SPSS. Results indicated that parents applied sunscreen most frequent and performed sun protection behaviours more often on younger children. Furthermore, indirect behaviours were frequently reported and demonstrated to be relative stable across children's ages. Parents reported that their children performed some sun protection behaviours themselves, which was particularly visible for older children and girls. A clear behavioural shift was only found for clothing behaviour, in planned situations. At the age of 11 children appeared to put on clothing more often than parents did for them. In order to predict potential other shifts at later ages, we used extrapolation. These results indicated that children appear to perform sun protection behaviours more often than their parents at the age of 14. Although the results reveal a large dependence on parental behaviours, the age shifts clearly stress the importance of targeting children in interventions. We further recommended specific attention to boys since they seemed to protect themselves less frequent than girls did. Moreover, this study indicated that an intervention focus on both girls and boys when entering the adolescent phase, seems warranted.

\section{Chapter 5}

In Chapter 5, findings from a study examining patterns of children's sun exposure and parental sun protection behaviours, in relation to sunburn occurrence, are outlined. By identifying separate groups of children based on behavioural patterns and sunburn, we aimed to understand children's risk of sunburn more closely. We used cross-sectional baseline data ( $n=1299$ at TO) from our longitudinal survey study to perform Latent Class Analyses (LCA) in SAS. Overall results indicated that sunburn in the previous year was frequent (>40\%) and that older children experienced sunburn more often. In addition, levels of sun exposure 
were relatively high, and more frequently reported in incidental than planned situations, which also holds for sun protection behaviours. LCA revealed four distinct classes of parents concerning their children's sunburn, sun exposure and parent's sun protection behaviours. Moreover, the majority of parents reported fair sun protection, in which younger children and children with more sensitive skin complexions were most frequently protected with sun protection behaviours. When comparing the groups, frequent sun exposure in planned situations (e.g. going to the beach) seemed to correspond with high sunburn risk, even when parents reported high sun protection behaviours. This study revealed a complexity regarding children's sunburn and indicated suggestions for warranted research.

\section{Chapter 6}

In Chapter 6, the results of a systematic review investigating the influence of the physical environment on children's sun safety is provided. We aimed to gather scientific evidence on effectiveness of existing multicomponent interventions targeting sun protection behaviours of children in outdoor settings. We adhered to PRISMA guidelines for conducting systematic reviews and used the PICOS tool to formulate systematic search strings. Four scientific databases (Psyclnfo, PubMed, Web of Science, Cochrane) and Google Scholar were used to systematically search the current literature on multi-component sun safety interventions. In total, 1085 articles were screened and after sequentially screening titles, abstracts and full-texts, 7 articles remained eligible for data abstraction. We reviewed the evidence of stand-alone effects on socio-cognitive determinants, sun protection behaviours, sun exposure, sunburn and nevi incidence. Results showed that 5 of the 7 studies indicated positive results of an environmental component. Free provision of sunscreen was most often the element of interest, however not showing consistent effectiveness. Shade provision seemed most promising for increasing shade-seeking behaviours, as was indicated in two included studies. This study accentuated the impact of the physical environment on evoking sun safety behaviours of both children, adolescents and parents. Moreover, minor recommendations regarding shaping outdoor environments were provided.

\section{Chapter 7}

Chapter 7 provides an overview and discussion of the presented studies in this thesis. Recommendations for future research on primary skin cancer prevention and practice are specified as well. 


\section{Samenvatting}


Huidkanker - bestaande uit basaalcelcarcinoom (BCC), plaveiselcelcarcinoom (PCC) en melanoom, is sinds het einde van de jaren ' 80 dramatisch toegenomen in Nederland en momenteel behoren wij tot de landen met de hoogste huidkankercijfers ter wereld. Huidkanker drukt een zware en alsmaar toenemende last op onze samenleving door onder andere hoge zorgkosten. De excessieve toename van huidkankercijfers in Nederland kan gedeeltelijk worden verklaard door vergrijzing, alsmede door veranderingen in ons gedrag met betrekking tot de zon. Aangezien huidkanker voorkomen kan worden door adequate zonbeschermende gedragingen toe te passen, zijn initiatieven om zonbescherming te verbeteren in onze samenleving noodzakelijk. Onbeschermde blootstelling aan de zon en zonverbranding(en), met name in de kindertijd, zijn de belangrijkste risicofactoren voor het ontwikkelen van huidkanker. Het beschermen van kinderen tegen de schadelijke effecten van de zon is daarom cruciaal. Een essentiële rol is weggelegd voor ouders en verzorgers, aangezien kinderen (nog) niet in staat zijn om zich zelfstandig tegen de zon te beschermen.

Zonbescherming bestaat uit meerdere, complexe gedragingen en kan bestaan uit het smeren van zonnebrandcrème, het aantrekken van beschermende kleding, een hoed en zonnebril en het tijdig opzoeken van schaduw. De toepassing van deze gedragingen is afhankelijk van verschillende factoren zoals de hoogte van de UV-index, het huidtype en de tijdsduur van blootstelling aan de zon. Momenteel laat internationale literatuur zien dat de zonbescherming van kinderen suboptimaal is, waardoor initiatieven om kinderen beter te beschermen uiterst noodzakelijk zijn. Ondanks dat huidkanker een omvangrijk probleem is in Nederland, is er weinig bekend over de mate van zonbescherming van kinderen. Het is daarom van groot belang om de huidige situatie met betrekking tot zonbescherming en zonblootstelling van kinderen in Nederland in kaart te brengen. Hierbij is het belangrijk dat zowel het ouderlijk perspectief als dat van kinderen zelf wordt meegenomen en dat de gedragingen worden onderzocht in- en tijdens verschillende situaties en activiteiten. Door beschermingsgedragingen van ouders te onderzoeken en te begrijpen, kunnen adviezen voor interventies die zonbeschermend gedrag bevorderen gegeven worden.

\section{Hoofdstuk I}

In Hoofdstuk 1 wordt een algemene introductie voor de studies in dit proefschrift gepresenteerd. Het doel van de studies was om bij te dragen aan huidige kennis over de mate van zonbescherming van kinderen in de leeftijd van 4 tot 12 jaar oud in Nederland, en om met die kennis aanbevelingen te kunnen doen voor zonbeschermingsinterventies. Voor dit proefschrift zijn verschillende zonbeschermingsgedragingen van zowel ouders als hun kinderen, in meerdere situaties, onderzocht middels een uitgebreide strategie. De zonbeschermde gedragingen die in dit proefschrift beschreven worden zijn onderverdeeld in: 1) directe gedragingen (door bijvoorbeeld zonnebrandcrème aan te brengen of een zonnebril op te zetten bij het kind); en 2) indirecte gedragingen (oftewel ondersteunen), door kinderen te leren hoe zij zelf(standig) zonbeschermend gedrag kunnen uitvoeren. Daarnaast is blootstelling aan de zon onderverdeeld in 1) bewuste situaties (door bijvoorbeeld naar het zwembad of strand te gaan); en 2) onbewuste situaties (door andere buitenactiviteiten te ondernemen zoals fietsen of buitenspelen).

\section{Hoofdstuk 2}

Het doel van Hoofdstuk 2 was om uitgebreid inzicht te krijgen in percepties van ouders over zonverbranding en zonblootstelling van hun kinderen en het uitvoeren van zonbeschermende gedragingen. Om deze onderwerpen te exploreren is een kwalitatieve interviewstudie gedaan. In totaal zijn 26 interviews 
uitgevoerd, waarvan 17 interviews met moeders waren, en er voor 17 meisjes geantwoord werd. Twee onderzoekers voerden de interviews uit, en transcribeerden en codeerden de interviewteksten. Bij het coderen werd een interbeoordelaarsbetrouwbaarheid van $d=.84$ bereikt. De resultaten lieten zien dat zonverbrandingen bij kinderen vaak gerapporteerd werden, ondanks dat ouders een zonverbranding regelmatig lijken te onderschatten. Situaties waarin zonverbrandingen het vaakst voorkwamen bleken daarnaast gerelateerd aan water(activiteiten) en beperkte schaduwfaciliteiten. Tijdens de interviews rapporteerden alle ouders de uitvoering van ten minste één beschermingsgedraging in zonsituaties, waarbij het gebruik van zonnebrandcrème evident het vaakst voorkwam. Daarnaast kwam onjuiste kennis over de UV-index en het correcte gebruik van zonnebrandcrème aan het licht, ondanks dat alle ouders aangaven zonnebrandcrème te gebruiken. Ouders noemden daarnaast regelmatig dat zij niet voldoende voorbereid waren op (een langer durende) zonblootstelling en leken de noodzaak om zonbescherming toe te passen te onderschatten tijdens - met name - onbewuste zonmomenten. Wat verder opviel was dat het merendeel van de ouders aangaf een positieve houding te hebben ten aanzien van een zongebruinde huid bij zowel henzelf als hun kind(eren), wat in sommige gevallen ertoe leidde dat zij minder zonbescherming toepasten. Op basis van deze studie werd geconcludeerd dat educatie over het correcte gebruik van zonnebrandcrème, het stimuleren van (het gebruik van) beschermende kleding en het tijdig zoeken van schaduw bevorderd zou moeten worden in interventies. Daarnaast behoren het creëren van fysieke omgevingen waarin zonbeschermend gedrag wordt aangemoedigd, door bijvoorbeeld schaduwplekken te faciliteren, als het modificeren van de positieve houding ten opzichte van een zongebruinde huid bij ouders, tot belangrijke elementen voor toekomstige zonbeschermingsinterventies.

\section{Hoofdstuk 3}

In Hoofdstuk 3 wordt een determinantenstudie beschreven waarin de relevantie van een uitgebreide set socio-cognitieve determinanten voor het voorspellen van zowel zonbeschermingsintenties als uitvoering van directe en indirecte zonbeschermingsgedragingen is onderzocht. Voor deze studie is longitudinale data van twee metingen gebruikt ( $n=904$ ouders tijdens T1 en $n=670$ ouders tijdens T2) om de CIBER-methodiek uit te voeren in statistisch softwareprogramma $R$. Deze methode visualiseert zowel univariate distributies (groepsgemiddelden) en associaties met uitkomstmaten (correlaties), waardoor de relevantie van de sociocognitieve determinanten voor zonbeschermingsintenties en -gedragingen kon worden onderzocht. In totaal zijn 27 generieke (zoals kennis en risico-percepties) en gedragsspecifieke (zoals sociale norm, of eigeneffectiviteit) determinanten getoetst. De resultaten indiceerden dat gedragsspecifieke determinanten het meest relevant waren in het voorspellen van zonbeschermingsintenties en gedragingen, waarbij met name attitude, eigen-effectiviteit en het formuleren van actieplannen de meeste variantie verklaarden. De ervaren eigen-effectiviteit onder ouders om zonbeschermend gedrag uit te voeren liet daarnaast veel ruimte voor verbetering zien, door lage groepsgemiddelden op deze determinanten. Bovendien waren het hebben van een positieve houding ten aanzien van een zongebruinde huid en (een) eerdere zonverbranding(en) van het kind negatief geassocieerd met beschermingsgedrag van ouders. De gehele set determinanten vertoonde de hoogste verklaarde varianties in schaduw zoekend gedrag en de laagste verklaarde varianties in ondersteunend gedrag. Samengevat heeft deze studie uitgebreid inzicht verschaft in de meest relevante socio-cognitieve determinanten gerelateerd aan zonbeschermende gedragingen van ouders, waardoor suggesties voor het ontwikkelen van toekomstige educatieve interventies geformuleerd konden worden.

\section{Hoofdstuk 4}

Hoofdstuk 4 beschrijft bevindingen over het uitvoeren van verschillende zonbeschermende gedragingen van zowel ouders als kinderen zelf, beiden op basis van zelfrapportage van ouders. Het belangrijkste doel van deze studie was om te onderzoeken of er een gedragsmatige verschuiving zou plaatsvinden - waarin kinderen zonbeschermend gedrag vaker uitvoeren dan hun ouders doen voor hen. Voor deze studie is cross-sectionele data ( $n=1053$ ouders tijdens T1) uit de longitudinale survey studie gebruikt om tweeweg 
ANOVA en lineaire regressies uit te voeren in statistisch softwareprogramma SPSS. De resultaten lieten zien dat ouders zonnebrandcrème het vaakst uitvoerden en alle zonbeschermende gedragingen vaker uitvoerden bij jongere, dan oudere kinderen. Daarnaast rapporteerden ouders indirecte (ondersteunende) gedragingen frequent hoog bij kinderen van alle leeftijden. Met betrekking tot gedrag van kinderen zelf bleek dat vooral oudere kinderen en meisjes in bepaalde mate zelfstandig zonbescherming uitvoerden. Enkel bij het aantrekken van beschermende kleding en attributen tijdens bewuste zonmomenten werd een verschuiving in gedrag gevonden, waar kinderen vanaf de leeftijd van 11 jaar dit gedrag vaker uitvoerden dan ouders dat voor hen deden. Door extrapolatie toe te passen werd daarnaast voorspeld dat kinderen overige zonbeschermende gedragingen vaker toe lijken te gaan passen dan hun ouders rond de leeftijd van 14 jaar. Ondanks dat deze studie liet zien dat kinderen nog in hoge mate afhankelijk zijn van het beschermingsgedrag van hun ouders voor hen, werd duidelijk dat zonbeschermingsinterventies ook op kinderen zelf dienen te focussen. Bovendien werd op basis van deze studie geadviseerd dat jongens specifieke aandacht verdienen in interventies aangezien zij minder zonbeschermende gedragingen lijken uit te voeren dan meisjes. Echter, wanneer de adolescentiefase begint, wordt een focus op zowel jongens als meisjes zeer belangrijk.

\section{Hoofdstuk 5}

In Hoofdstuk 5 wordt een studie besproken waarin gedragspatronen van ouders, in relatie tot zonverbranding(en) bij hun kinderen, geëxploreerd zijn. Het doel van deze studie was om zonverbrandingen bij kinderen beter te begrijpen, door verschillende groepen kinderen te identificeren en te differentiëren op basis van hun zonverbranding(en), mate van blootstelling aan de zon en beschermingsgedragingen van hun ouders voor hen. Voor deze studie is cross-sectionele data ( $n=1299$ ouders tijdens TO) uit de longitudinale survey studie gebruikt om Latent Class Analyses (LCA) uit te voeren in de statistische softwareprogramma's $R$ en SAS. De resultaten lieten zien dat zonverbrandingen in het afgelopen jaar vaak voorkwamen bij kinderen $(>40 \%)$ en dat oudere kinderen vaker door de zon verbrandden dan jongere kinderen. Daarnaast rapporteerden ouders een relatief hoge mate van zonblootstelling en rapporteerden zij vaker het gebruik van zonnebrandcrème en het opzoeken van schaduw tijdens bewuste zonmomenten en het gebruik van beschermende kleding tijdens onbewuste zonblootstelling. De analyses onthulden vier groepen ouders op basis van zonverbranding, zonblootstelling en zonbescherming van en bij hun kind. De meerderheid van de ouders rapporteerde een redelijk hoge frequentie van beschermingsgedragingen, waarbij met name jongere kinderen en kinderen met de meest sensitieve huidtypes het vaakst beschermd werden. Bij het vergelijken van de vier groepen kwam verder naar voren dat frequente zonblootstelling tijdens bewuste zonmomenten geassocieerd leek te zijn met een hoger risico op zonverbranding, zelfs wanneer ouders een hoge frequentie van beschermende gedragingen rapporteerden. De complexiteit van zonverbrandingen bij kinderen is door deze studie verder onthuld, waardoor suggesties voor nader onderzoek over dit cruciale onderwerp zijn gegeven.

\section{Hoofdstuk 6}

In Hoofdstuk 6 wordt een systematische review studie over wetenschappelijk bewijs voor de invloed van de fysieke omgeving op het uitvoeren van zonbeschermende gedragingen beschreven. Het doel van deze studie was om inzicht te verkrijgen in de effectiviteit van internationaal bestaande interventies die gericht zijn op het bevorderen van zonbescherming onder kinderen en adolescenten in verschillende settings. De PRISMA-richtlijnen voor het uitvoeren van systematische reviews werden gevolgd en de PICOS-tool werd gebruikt om systematische zoekopdrachten te formuleren voor verschillende databases. Vier wetenschappelijke databases (PsycInfo, PubMed, Web of Science en Cochrane) en Google Scholar werden gebruikt om systematisch door de huidige wetenschappelijke literatuur te zoeken. In totaal werden 1085 artikelen gevonden en gescreend. Na het aansluitend bestuderen van studies op basis van titels, abstracts en volledige manuscripten bleven 7 wetenschappelijke studies over die de effecten van 
omgevingsinterventies beschreven. Het bewijs voor de effecten van deze interventies op socio-cognitieve determinanten, zonbeschermende gedragingen, zonblootstelling, zonverbranding(en) en moedervlekken werd vervolgens kritisch geëvalueerd. De resultaten toonden dat 5 van de 7 studies positieve effecten van een omgevingsinterventie lieten zien. Het gratis verspreiden van zonnebrandcrème werd het vaakst gebruikt als interventiecomponent, maar liet geen consistente effectiviteit op het gebied van de uitkomstmaten zien. Het faciliteren van schaduwplekken leek het meest effectief voor het bevorderen van schaduw zoekend gedrag, wat naar voren kwam in 2 studies. Deze review studie benadrukt de impact die de fysieke omgeving kan hebben in het uitvoeren van zonbeschermend gedrag van kinderen, adolescenten en ouders. Op basis van de resultaten zijn suggesties gegeven voor het vormgeven van fysieke omgevingen.

\section{Hoofdstuk 7}

Tot slot geeft Hoofdstuk 7 een overzicht en bediscussiëring van alle gepresenteerde studies, met het oog op de huidige wetenschappelijke literatuur. Daarnaast worden in dit laatste hoofdstuk wetenschappelijke en praktische aanbevelingen gedaan voor toekomstig onderzoek in het kader van primaire huidkankerpreventie en interventieontwikkeling in Nederland. 
170 Breaking through the clouds 
Curriculum Vitae 


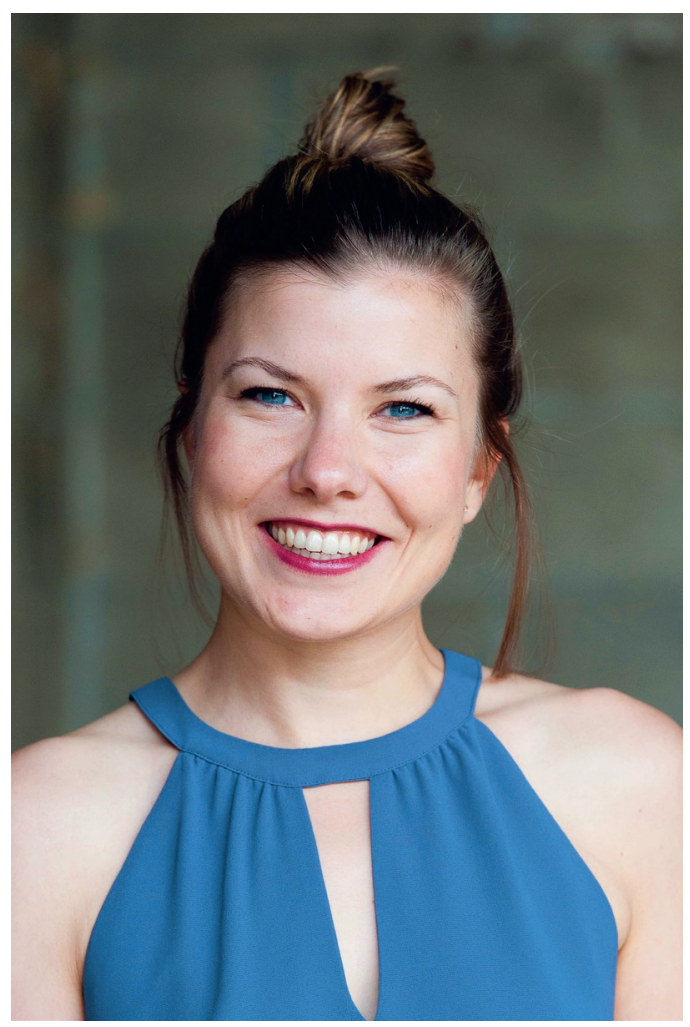

\section{Karlijn Thoonen}

Place of birth: Elst, the Netherlands

Date of birth: 28th January 1991

Bachelor's: Applied Psychology

Master's: Mental Health

Karlijn Thoonen was born in Elst (Gelderland), the Netherlands, on January 28, 1991. After high school graduation, she moved to Eindhoven and started a Bachelor in applied psychology. She wrote her bachelor thesis on the development of a physical activity programme for patients with psychotic disorders. In 2015, she finished a Master's degree in clinical psychology at Maastricht University. Her master thesis focused on the effectiveness of cognitive-behavioral therapy (CBT) and CBT in combination with schema focused therapy (ST) among patients with a personality disorder, depression, and anxiety.

After University, Karlijn worked as a mental health therapist in a clinical setting. In 2016 she went travelling and volunteering in Southeast Asia for six months and decided to pursue a job in academia instead.

Directly after returning to the Netherlands, she started as a researcher at the department of Health Promotion at Maastricht University on a collaborative project with the Dutch Cancer Society (Amsterdam). This project concerned both support for tobacco control policies in the Dutch population and primary skin cancer prevention among children and their parents. During her PhD, Karlijn was active as a board member of the department's daily board and temporary employees board, involved in teaching and supervising graduate students in Bachelor's and Master's programmes in psychology and health sciences, co-writing grant proposals and attending several professional courses and workshops. 
Publication list

\section{Publication list}




\section{Publications in this thesis (d.d. 8th March 202I)}

Thoonen, K., Schneider, F., Candel, M., de Vries, H., \& van Osch, L. (2019). Childhood sun safety at different ages: relations between parental sun protection behavior towards their child and children's own sun protection behavior. BMC Public Health, 19(1), 1044. doi: 10.1186/s12889-019-7382-0

Thoonen, K., van Osch, L., de Vries, H., Jongen, S., \& Schneider, F. (2020). Are Environmental interventions targeting skin cancer prevention among children and adolescents effective? A Systematic Review. International Journal of Environmental Research and Public Health, 17(2), 529. doi: 10.3390/ijerph17020529

Thoonen, K., van Osch, L., Drittij, R., de Vries, H., \& Schneider, F. (2021). A Qualitative Exploration of Parental Perceptions Regarding Children's Sun Exposure, Sun Protection, and Sunburn. Frontiers in Public Health, 9:596253. doi: $10.3389 /$ fpubh.2021.596253

Thoonen, K., Lima Passos, V., Schneider, F., de Vries, H., \& van Osch, L. (2021). Children's sunburn exposed: Identification of sun exposure and parental sun protection patterns. Manuscript accepted for publication (d.d. 2th Feb 2021) in European Journal of Dermatology. doi (preprint): 10.31234/osf.io/fcz5b

Thoonen, K., van Osch, L., Crutzen, R., de Vries, H., \& Schneider, F. Identification of relevant socio-cognitive determinants explaining multiple parental sun protection behaviors. Manuscript under review (d.d. 15th Aug 2020) for publication in Health Education and Behavior. doi (preprint): 10.31234/osf.io/yuqzd

\section{Reports}

Thoonen, K., Willems, M., van Osch, L., de Vries, H., \& Schneider, F. (2017). Rapport 'Rookvrij Nederland': Eerste nameting. Maastricht: Vakgroep gezondheidsbevordering. Report issued to the Dutch Cancer Society.

Thoonen, K., Willems, M., Schneider, F., de Vries, H., \& van Osch, L. (2017). 'Slim in de Zon': Eerste nameting. Maastricht: Vakgroep gezondheidsbevordering. Report issued to the Dutch Cancer Society.

Thoonen, K., van Lierop, M., van Osch, L., de Vries, H., \& Schneider, F. (2017). Rapport 'Rookvrij Nederland': Tweede nameting. Maastricht: Vakgroep gezondheidsbevordering. Report issued to the Dutch Cancer Society.

Thoonen, K., van Lierop, M., Schneider, F., de Vries, H., \& van Osch, L. (2018). 'Slim in de Zon': Tweede nameting. Maastricht: Vakgroep gezondheidsbevordering. Report issued to the Dutch Cancer Society.

Thoonen, K., van Lierop, M., van Osch, L., de Vries, H., \& Schneider, F. (2018). Rapport 'Rookvrij Nederland': Derde nameting. Maastricht: Vakgroep gezondheidsbevordering. Report issued to the Dutch Cancer Society.

Thoonen, K., van Lierop, M., van Osch, L., de Vries, H., \& Schneider, F. (2018). Rapport 'Rookvrij Nederland': Vierde nameting. Maastricht: Vakgroep gezondheidsbevordering. Report issued to the Dutch Cancer Society.

Thoonen, K., van Osch, L., van Lierop, M., de Vries, H. \& Schneider, F. (2018). Rapport additionele studie 'Zonbeschermingsbeleid op recreatieve plekken in Nederland'. Report issued to the Dutch Cancer Society.

Thoonen, K., van Lierop, M., Schneider, F., de Vries, H. \& van Osch, L. (2018). Rapport additionele studie 'Literatuuronderzoek zonbeschermingsinterventies op recreatieve plekken'. Maastricht: Vakgroep gezondheidsbevordering. Report issued to the Dutch Cancer Society. 
Thoonen, K., Winkens, B., Schneider, F., de Vries, H., \& van Osch, L. (2019). Eindrapportage 'Slim in de Zon'. Maastricht: Vakgroep gezondheidsbevordering. Report issued to the Dutch Cancer Society.

\section{Presentations at (international) conferences}

\section{UV \& Skin cancer prevention conference, 2018, Toronto, Canada}

\section{Oral presentations:}

Determinants of supportive sun protection behavior of parents and the relationship with sun protective behaviour of the child, and;

Parental sun protective behavior during intentional and unintentional sun exposure: a longitudinal study Poster: Support for sun protection policies in the Netherlands among parents of primary school children

\section{CAPHRI Research Day, 2018, Maastricht, the Netherlands}

\section{Oral presentation:}

Evaluations of mass media campaigns concerning tobacco control and sun safety. A collaboration between the Dutch Cancer Society \& Maastricht University

\section{Association for Researchers in Psychology and Health (ARPH) Conference, 2019, Egmond aan Zee, the Netherlands}

\section{Oral presentation:}

A closer look into sunburn prevention: Age and sex effects on parental and children's sun safe behaviour

\section{Association for Researchers in Psychology and Health (ARPH) Conference, 2020, Egmond aan Zee, the Netherlands}

\section{Oral presentation:}

Which determinants are most relevant and changeable in parental sun protection behaviors towards their children? An application of the CIBER method

\section{UV \& Skin cancer prevention conference, 2020, Mechelen, Belgium (postponed to 2021)}

\section{Symposium organizer and presenter:}

Childhood sun safety: From problem to solution 
180 Breaking through the clouds 
Dankwoord 


\title{
Het dankwoord is by far het meest gelezen onderdeel van elk proefschrift. Degenen die mij inmiddels (enigszins) kennen, weten dat ik royaal de tijd en ruimte genomen heb om in de pen te klimmen en woorden van dank en bewondering op te schrijven. Voor allen die nu (nog) schrikken; ik heb gebruik gemaakt van het feit dat aan dit onderdeel - voor de verandering - géén woordenlimiet vastzit, so fasten your seatbelts.
}

\begin{abstract}
Liesbeth en Francine, Francine en Liesbeth. Het was onmogelijk om de volgorde te bepalen waarin jullie naar voren zouden komen in dit dankwoord. Ondanks dat jullie op een hele andere, eigen manier van betekenis zijn geweest voor mij en mijn project, staan jullie volledig gelijk in het stukje dank dat ik naar jullie wil uitspreken. Ik herinner me de eerste keer dat ik jullie ontmoette - via een scherm, terwijl we zo'n 10.000 kilometer van elkaar verwijderd waren - nog als de dag van gisteren. Jullie zaten naast elkaar, op de achtergrond in Hein's kantoor, en ik vond jullie meteen heel opgewekt, vriendelijk en energiek over komen. Daarnaast voelde ik meteen een klik, ook al was deze online. Toen ik jullie wat weken later in het echt ontmoette, werd ik omvergeblazen door de vaart waarin jullie communiceerden en hoezeer jullie op elkaar ingespeeld waren. Gelukkig wende dat snel en werd ik ook onderdeel van het team!
\end{abstract}

Francine, als ik iets van jou geleerd heb dan is het wel inventief, creatief en positief zijn. Jouw vermogen om 'om te denken' is echt bewonderenswaardig. Je hebt de capaciteit om een positieve helicopterview aan te nemen en je niet te verliezen in details - hoe uitdagend een situatie ook kan zijn. Ook ben jij als geen ander in staat om orde te scheppen in chaos. Ondanks dat je kritisch was op mijn stukken, kreeg ik geregeld inspiratie door jouw manier van feedback geven. En wanneer ik zelf nog niet overtuigd was van een originele methode, de veelomvattendheid van onze data of de kwaliteit van een stuk dat ik op papier had gezet, dan was er altijd een leuk mailtje van jou dat me nèt even dat zetje gaf om in te zien dat ik op de goede weg was. Dit bracht ook een wisselwerking teweeg; ik werd enthousiaster over onze studies, wat jouw begeleiding in mijn project waarschijnlijk leuker maakte. Ook heb ik altijd heel sterk ervaren dat jij nooit te beroerd was om me aan te horen of samen te sparren over (lastige) materie. Ik heb daarnaast veel geleerd van jouw 'planmatige' en systematische manier van werken, wat het vaakst tot uiting kwam in het opbouwen van introducties of duiden van resultaten. Ondanks dat ik me altijd een vreemde eend in de bijt gevoeld heb met mijn achtergrond in Mental Health, heb jij me weten te enthousiasmeren en te laten groeien in het onderzoeksveld van Health Education \& Promotion. Jouw bezieling maakt je een superkracht voor het onderwijs en voor studenten, en ik heb daar ook zeker van mogen meegenieten als promovendus. Daarnaast heb ik veel gehad aan jouw relativeringsvermogen, vooral toen ik sterk twijfelde over het voortzetten van mijn project. Je wilde niet jouw mening opleggen en gaf mij de ruimte om zelf dichterbij een besluit te komen. Je kon mij een realistisch beeld geven over het doen (en afronden) van een $\mathrm{PhD}$ en liet ook merken dat je je voor mij in wilde zetten. Je hebt altijd gezegd dat het laatste jaar van een (maar vooral mijn) project het allerleukste zou zijn en daar heb je voor de volle $100 \%$ gelijk in gehad. Plus, jouw enthousiasme en al onze zoom-meetings in dit laatste jaar waren ècht de kers op deze taart! Francine, bedankt voor alles en nog veel meer!

Liesbeth, Enne? Ik zal vast niet de eerste zijn die dit zinnetje in het dankwoord plaatst achter jouw naam. Met deze alom bekende vraag startte elk van onze overleggen, maar het duurde een tijdje voordat ik begreep wat ik er allemaal op kon antwoorden. De lijst met typische 'Liesbeth' kenmerken wordt daarnaast gevuld met jouw onmiskenbare (snelle) loopje door de wandelgang, je ontzettend stijlvolle garderobe, jouw veelomvattende vocabulaire en het vermogen om je heel sterk, zorgvuldig en professioneel uit te drukken, de 'Anyway...' tijdens onze overleggen, de 'Knock Knock' als je even snel het kantoor binnen kwam, jouw oog voor detail en natuurlijk de namiddag-ronde richting de eet- en drankautomaten beneden op Deb of het MUMC omdat je nog geen tijd had gehad om te lunchen. Hiernaast heb je mij ook op een hele fijne manier begeleid in de afgelopen jaren. Ik heb altijd het gevoel gehad dat ik bij jou binnen kon lopen hoe druk je ook was. Het was fijn dat je mij altijd serieus nam en vaak begrip toonde voor een lastige batch aan analyses, strubbelingen met data-interpretatie of moeilijkheden met het krijgen van overzicht. Jouw opmerkzaamheid, de scherpte waarmee jij elk stuk bekijkt en interpreteert en jouw vermogen om verbanden te leggen heb ik altijd erg bewonderd - en ik heb er veel van geleerd. Je zag altijd ruimte voor nieuwe en interessante onderzoeken en kansen, wat bij mij zorgde voor meer bevlogenheid. Soms kon ik er 
last van hebben dat een stuk maar niet goed of af leek te zijn, maar jouw kritische blik hebben veel van mijn stukken stukken beter gemaakt. Liesbeth, ontzettend bedankt voor jouw fijne begeleiding en het zijn van een inspiratiebron!

Hein, bij de ontmoeting die ik hierboven omschrijf was jij natúúrlijk ook aanwezig. Ik zie het tafereel nog steeds voor me; jij wat prominent in beeld aan het bureau in jouw kantoor - waar ik in de jaren erna nog veel zelf zou komen te zitten tijdens onze overleggen, met op de achtergrond Liesbeth en Francine. Het werd mij vrij snel duidelijk dat je rechtuit en bevlogen communiceert en veel belang hecht aan toegankelijkheid. Ik heb dan ook aan het einde van veel overleggen moeten (en mogen) lachen om jouw humor. Door de jaren heen, maar vooral toen het KWF project erop zat, ben ik je gaan leren kennen als een optimistisch, zeer ondernemend, veelzijdig en gedreven persoon. Ondanks dat ik in het begin van mijn project overrompeld kon zijn door jouw manier van werken, heeft het me ook heel veel opgeleverd en waardeerde ik het dat jij eerlijk en direct was. Ook was het altijd heel prettig dat jij, wanneer er wat hobbels op de weg waren, een rooskleurig alternatief kon bieden. Waar ik in het begin van mijn project schrok als jij opmerkingen maakte als: 'Straks tijdens je verdediging moet je dit kunnen beantwoorden', leerde ik dat jij dit als stimulans bedoelde om extra kritisch na te denken. Nou, ik heb er alle vertrouwen in dat ik mijn verdediging goed ga volbrengen na alle stof tot nadenken die jij mij gegeven hebt! Ik blijf het bijzonder vinden dat we pas sinds COVID-19 van elkaars passie voor piano af weten en hoop dan ook dat we toch nog eens samen een stukje Einaudi, Tiersen of Beethoven kunnen spelen. Ergens is het heel bijzonder dat we door het noodzakelijke thuiswerken ook afsluiten zoals we in 2016 begonnen zijn; via een beeldscherm. Hein, heel veel dank voor alle wijsheid, positiviteit en aanmoediging in de afgelopen jaren!

Graag wil ik ook de leescommissie bedanken voor het beoordelen van dit proefschrift. Ondanks dat jullie pas aan het einde van dit proefschrift een rol hebben gespeeld, zijn jullie wel onmisbaar geweest. Heel veel dank voor alle tijd en energie die jullie gestoken hebben in het lezen van mijn proefschrift; Stef, Jessica,

\section{Rob, Lilian en Benedicte.}

Veel dank ook aan de co-auteurs en statistici van papers die in dit proefschrift opgenomen zijn! Alle studies die gedaan zijn waren nooit van deze kwaliteit geweest zonder jullie hulp. Rowan, jij hebt heel goed geholpen bij het opzetten, uitvoeren én interpreteren van de kwalitatieve studie. Ik had me geen fijnere collega kunnen wensen; je pakte alle onderdelen zó goed op en had een sterke intrinsieke motivatie om alles uit deze leuke studie te halen. Ik vond het heel leuk om met jou deze studie uit te voeren, vooral omdat ik na 3 jaar onderzoek doen juist het stukje van samenwerken echt begon te missen. Bedankt voor al je inzet en positiviteit! Ik wens je heel veel succes en geluk in jouw toekomst! Rik, ik vond het - op z'n zachtst gezegd - erg leuk om met jou samen te werken aan het 'CIBER paper' en heb het door jouw enthousiasme heel leuk gevonden om al die plots uit te draaien, te finetunen en te interpreteren! Ik had natuurlijk al heel veel sneak peaks van jouw werkstijl gehad via Esther en in het DB en de blokplanningsgroep van Alcohol \& Drugs, maar toch was ik verrast over hoe goed je kunt relativeren en enthousiasmeren. Ik vond het ook heel leuk dat je me de kans gaf om in jouw plaats een presentatie te geven over de CIBER resultaten op de ARPH conferentie. Ik ken niemand die zo bevlogen zijn werk doet en daarnaast zo geduldig, optimistisch en innemend is. Veel dank voor het zijn van zo'n leuke collega en al je hulp! Stefan, ook jij mag zeker niet in dit rijtje ontbreken. Ik kijk heel prettig terug op onze samenwerking. Het was voor mij een verademing om met iemand te kunnen sparren over het opzetten van search strings en te beslissen welke databases het meest geschikt zouden zijn. Jij wist overduidelijk waar je over sprak en dat hielp mij om enthousiaster te worden over het doen van een systematische review. Ook vond ik het heel leuk om van jou te horen hoe jij een PhD ervaren had; met alle ups en downs die daarmee gepaard gaan en dus ook heel normaal zijn. Stefan, heel veel dank! Math, jij bood statistische hulp bij mijn eerste studie. Het was heel fijn om te kunnen brainstormen over analysemogelijkheden en zonder jouw scherpe blik was het me niet gelukt om het paper tot een hoger niveau te tillen. Bedankt voor al het geduld en het kritische meedenken! Bjorn, wat was het fijn om met jou (en Vera) samen de analyses voor het eindrapport van KWF op te zetten. Jouw engelengeduld en vermogen om complexe materie eenvoudig uit te leggen waren heel prettig. Je was daarnaast erg betrokken bij de analyses, wat zich uitte in een 'uitleg-meeting' voor ons hele team en het regelmatig binnenwandelen in mijn kantoor om nog iets te verduidelijken. En, zonder deze samenwerking had ik nooit jouw leuke kamergenoot ontmoet, dus dubbel dank! Valeria, Bjorn's great roommate! How I wish I had met 
you sooner, since it was such a delight to work with you - to say the least! I really enjoyed working together on the Latent Class paper, which took almost a year in total. You consistently took a lot of time and effort to explain me or help me with statistics and interpretation of the results. I always felt at ease with you and loved our conversations about your life, my life, and working life in academia. I really appreciated your working style; by being pragmatic, supportive and inspirational, working with you was real fun! To me, you are a great example of a power woman. Thank you $V$, and: groetjes!

Heel veel dank voor de onderzoeksassistenten die geholpen hebben om de 'Brede Effectmeting' tot een goed einde te brengen; Marion, Merel en Vera. Zonder jullie inzet was het mij zeker niet zomaar gelukt om alle KWF rapporten (op tijd) te schrijven! Vera, zonder jouw hulp tijdens het eindrapport weet ik niet hoe ik uit de wirwar van multilevelanalyses had kunnen komen. Gelukkig vond jij het ook nog eens heel leuk om te doen. Bedankt! Marion, wij hadden vanaf het begin al heel veel gemeenschappelijk; beiden uit Gelderland, TP gestudeerd in Eindhoven en daarna naar Maastricht gegaan om verder te studeren, en beiden de ambitie om een PhD te gaan doen! Het was dan ook erg leuk dat jij mij kwam helpen met mijn project. We hadden een hele leuke klik en dat kwam zeker ten goede aan ons werk. Al snel ging je zelf starten met jouw promotie en moesten we vervanging regelen. Marion, ik vind je een geïnteresseerd en integer persoon en het is daarom altijd heel leuk om met je bij te praten. Laten we vaker gaan wandelen op de Strabregtse heide met een kannetje thee en een plak cake! Heel veel succes met het afronden van jouw proefschrift dat gaat zéker goed komen - en heel veel dank voor al je steun en interesse in de afgelopen jaren!

Binnen de 'Brede Effectmeting' heb ik veel mogen samenwerken met KWF Kankerbestrijding en TNS-KANTAR. Ik heb heel veel geleerd van deze twee organisaties door alle overlegmomenten en praktijkbezoeken. Een aantal personen zou ik graag in het bijzonder willen bedanken. Sabine, jij leverde altijd ontzettend snel een gloednieuwe dataset aan wanneer ik een extra verzoekje had om een variabele toe te voegen en je was altijd bereid om extra tijd te besteden aan mijn vragen/telefoontjes over de complexe datasets. Ik vond het heel fijn en helder samenwerken met jou; heel veel dank! Laura, lliaz en Andrea, met jullie heb ik heel wat overlegmomenten gehad over 'Rookvrij Nederland' of 'Slim in de Zon'. Ondanks dat het soms zoeken was naar consensus, vond ik het erg leuk om van jullie te leren en een kijkje in jullie 'keuken' te krijgen. Veel dank voor de inspiratie die jullie mij gegeven hebben en de gastvrijheid van jullie gehele kantoor in Amsterdam om mij wegwijs te maken in jullie wereld! Bart en Kim, jullie noem ik nog even in het bijzonder. Ik denk nog vaak terug aan de leuke momenten die we hebben gehad tijdens het UV-congres in Toronto. In mijn geval was dit het eerste buitenlandcongres en ik vond het heel fijn dat ik niet de enige Nederlander was die daar rondliep. Het samen in een sessie zitten en elkaar aanmoedigen voor onze presentaties was echt heel erg leuk! Bart, jou ben ik na het congres nog vaker tegen gekomen op Nederlandse congressen of mijn bezoekjes aan KWF. Het was dan altijd erg gezellig om met je bij te praten en te sparren over de toekomst van huidkankerpreventie in Nederland! Kim, je bent altijd bereid om te overleggen of mee te denken over boeiende UV-gerelateerde (onderzoeks)onderwerpen. Mede dankzij jou ben ik me steeds meer gaan interesseren voor dit belangrijke onderwerp, waarvoor veel dank!

En dan natuurlijk de collega's van de GVO vakgroep! Door de jaren heen zijn er verschillende collega's gekomen en gegaan en met velen van hen heb ik leuke momenten gehad! Een aantal collega's wil ik in het bijzonder bedanken. Stef, als hoogleraar, toen vakgroepvoorzitter en daarna medelid van het dagelijks bestuur heb ik je op verschillende manieren mogen leren kennen. Het is overduidelijk dat je een ontzettend warm hart toedraagt aan onze vakgroep. Ik waardeerde het altijd heel erg dat je - buiten de professionele kant - een hele informele en toegankelijke kant hebt. En natuurlijk vond ik het altijd leuk dat er een medeEindhovenaar (ook al zijn we beiden geen échte) in Maastricht zat! Dankzij jou was er veel gezelligheid op de vakgroep, waarvoor dank! Kim, Patricia, Daisy en Denise, dankzij jullie werd het werk altijd net even wat makkelijker of gezelliger! Bedankt voor alle hulp en gezellige praatjes. Kim, jou wil ik in het bijzonder noemen; je was altijd erg begripvol, geïnteresseerd en betrokken en dat vond ik erg fijn. Ik vind je echt een topper en ik hoop dan ook dat je precies blijft zoals je bent! Leon, wat hebben wij (Esther en ik) veel gelachen met jou. Natuurlijk denk ik meteen aan carnaval, maar buiten dat jaarlijkse feestje was er nog genoeg ander leuks te beleven. Jouw deur stond altijd open en je bent ook altijd in voor een praatje, wat ik erg leuk vond. En, volgend jaar zijn wij gewoon weer van de partij hoor, tijdens carnaval 2022! Sacha en Nicole, samen hebben we zo'n twee jaar het TMO-board gevormd. Jullie waren al even voor mij gestart met jullie PhD en 
wisten dus al wat meer van onze vakgroep, wat mij weer hielp om een plekje te vinden binnen de grote groep van collega's. Ondanks dat we niet altijd bereikten wat we hoopten, kijk ik leuk terug op die tijd; bedankt! Adriana, je was altijd erg nieuwsgierig naar mijn project en heel betrokken bij wat ik deed. Ik vond onze koffietjes gezellig en wellicht dat we de gemiste koffies van afgelopen jaar nog een keer in real life kunnen inhalen. Heel veel dank en succes met de afronding van jouw project! Dennis, het was altijd leuk kletsen met jou over - vooral - hardlopen en intermittent fasting (doen jullie het nog steeds?) maar ook over inhoudelijke zaken zoals het doen van een systematic review - ik was destijds héél blij dat ik een voorbeeld aan jouw proces kon nemen! Ik heb je altijd erg gewaardeerd als collega en dan vooral dankzij jouw enthousiasme, inzet op de vakgroep en gezelligheid! llona, eigenlijk heb ik jou pas beter leren kennen aan het eind van 'onze' PhD's. We liepen bijna exact gelijk op en kregen uiteindelijk zelfs op dezelfde dag onze goedkeuring! Het was heel fijn om elkaar te updaten over het laatste schrijfproces wat vanuit huis moest gebeuren - en alle aarzelingen en strubbelingen die daarbij horen. Veel dank daarvoor! Stan, ik hoor nóg jouw bal door de gang heen vliegen, ook al heeft dit al zo'n twee jaar niet meer plaatsgevonden. Wat kijk ik leuk terug op de beginjaren waarin jij ons kantoor vaak kwam vergezellen - of het nou was om eten te stelen, Amsterdams te praten en zingen of toch echt om iets inhoudelijks. Zodra de deur dicht ging hoefden we ons niet meer in te houden, totdat Carolin op de muur bonkte natuurlijk. Bedankt Stan, voor alle gezelligheid, maar ook zeker voor jouw motiverende woorden toen ik mijn project even niet meer zag zitten. Jouw vertrouwen in mij heeft me destijds veel positieve spirit gegeven! Thomas, ik heb nog steeds de powerpuff-girl bewaard die je mij gaf toen ik je pas net een week kende. Wat hebben we leuke en fijne gesprekken gehad door de jaren heen waarin we zoveel herkenning vonden bij elkaar! We zijn hele verschillende types maar we kunnen goed samen filosoferen. Je bent echt een top-wetenschapper en ik weet zeker dat je een hele mooie carrière tegemoet gaat na je PhD; je hebt in ieder geval alles in huis! Dankjewel Thommie! Yil, jij bent de 'newbie' van de collega's die ik hier noem. Ik heb je in relatief korte tijd leren kennen als een ontzettend lieve, betrokken en gezellige collega. Je werkt ontzettend hard en bent nooit te beroerd om iemand anders te helpen. En, wat was het leuk om samen met jou paranimf te zijn bij Esther's verdediging! Heel veel succes met de laatste fase(s) van jouw PhD; ik weet zeker dat je het gaat rocken!

Joleen, jij hebt mij in de laatste fase van mijn promotie héél veel gebracht - veel meer dan ik aanvankelijk had kunnen bedenken. Ik wil je dan ook bedanken voor het bieden van alle steun, de inzichten en het luisterend oor!

Stijn, dankzij jou ligt dit proefschrift er ook daadwerkelijk, helemaal zoals ik het gewild heb. Ik vond het super leuk om samen 'op onderzoek' uit te gaan wat nou een passend design zou worden en ik ben heel blij met het eindresultaat; het klopt gewoon! Heel veel dank voor jouw bewonderenswaardige werk!

En dan familie en vrienden; zonder jullie had ik dit hele traject (en dan vooral op persoonlijk vlak) nooit zo goed getrokken. Jullie hebben elk op jullie eigen manier bijgedragen aan mijn proces.

Papa, ik weet bijna zeker dat jij de enige persoon bent die letterlijk elk woord van mijn proefschrift gelezen heeft; ik heb mijn nieuwsgierigheid absoluut van jou geërfd. Je kreeg zelfs zo'n drive van het (als eerste) ontvangen van mijn papers, dat een vertaalprogramma je beste vriend werd en je op eigen houtje mijn geschreven werk ging vertalen. Ik gók dat ik het bewandelen van mijn eigen pad, op mijn (eigen) (wijze) manier ook van jou heb mogen erven. Ik zal nooit de reactie vergeten die je gaf toen ik voorstelde je 'mee te nemen' naar Toronto omdat ik mocht gaan presenteren op het UV-congres. Daar gingen we samen een paar maanden later; op naar Canada en de States! Jij mocht als vader aanwezig zijn bij een van mijn presentaties en je vond het geweldig - en ik uiteraard ook. Volgens mij heb je tijdens de andere congresdagen een record gevestigd in het kader van 'zo snel als kan zoveel mogelijk musea bezoeken'. Jij weet inmiddels meer over Toronto dan de gemiddelde Canadees. Ik kijk met veel plezier terug op de vader-dochter trip; dat pakken ze ons nooit meer af! lk weet dat je heel trots op me bent en dat doet me heel goed. Bedankt voor alle interesse en betrokkenheid! Mama, ondanks dat je in tegenstelling tot papa niet elke letter gelezen hebt - wat ik ook ècht niet verwacht, weet ik ook dat jij erg betrokken was bij mijn onderzoek. Zodra er in de Gelderlander of Volkskrant een artikel stond dat ook maar enigszins te maken had met huidkankerpreventie, stuurde je het me door. Je hebt me natuurlijk ook geholpen bij het werven van basisscholen en hebt stad en land (op internet) afgezocht om een goede referentie te vinden over de 'bouwen' in het primaire onderwijs. 
Als we het hebben over gedragsverandering ben jij degene die ik sowieso al op mijn conto kan schrijven; álle zonbeschermende adviezen die belangrijk zijn, voer jij perfect uit! Waar ik nog niet met een pet of hoed over straat loop, draag jij al een zonnehoed met verve! Met regelmaat heb je de lijfspreuk van oma Thoonen; 'Je ergens niet toe kunnen zetten, is ook een vorm van niet kunnen' genoemd, welke uiteindelijk heel toepasselijk is geweest in mijn traject. Ik weet dat je heel trots op me bent. Het feit dat jij vereerd was toen ik vroeg of ik op jouw verjaardag mocht verdedigen, zegt alles! Bedankt mam!

Tim, mijn grote broer, maar eigenlijk ben ik soms ook gewoon jouw grote zus(je). Ik vond het altijd leuk hoe jij liet blijken dat je trots op me bent; door bijvoorbeeld héél vaak te zeggen dat het jou nooit gelukt zou zijn. Of dat, wanneer je iets van mij las, het je begon te 'duizelen'. Nou, als er één ding is dat wij gemeen hebben is het wel doorzettingsvermogen, dus ik ben er echt niet van overtuigd dat jij een PhD niet gekund zou hebben. Toen ik even niet meer wist of ik door wilde gaan kon ik het hier goed met jou en Renee over hebben. Jullie zouden de keuze - welke ik dan ook zou maken - sowieso begrijpen. Nu is het dan toch echt bijna zo ver en is je kleine zusje bijna dr. - there's no way back! Heel veel dank voor je steun, trots en vertrouwen in mij! En ik ben heel trots op jou! Renee, lieve schoonzus. Ik heb de afgelopen jaren heel wat kaartjes van jou en jullie verzameld; vol met lieve en aanmoedigende woorden. Je bent zowel dichtbij als op afstand vaak een bron van vertrouwen geweest voor mij. Je hebt altijd in mijn kunnen geloofd en me dat ook vaak laten weten. Ik vond het heel leuk en fijn dat je vaak zo oprecht geïnteresseerd was in mijn studies, en zo trots was als ik iets gepubliceerd had. Als ik ergens bescheiden over was, was jij er wel om mij juist extra trots te laten zijn op mezelf! Ik kan me geen leukere schoonzus wensen dan jij en ik hoop dan ook dat je nog jaren bij de Thoontjes zal blijven! En, blijf vooral (dicht bij) jezelf, want dan ben je het allerleukst. Bedankt voor alles Reneetje!

Mieke, als er iémand is die al vroeg zag dat ik moest gaan schrijven, was jij het wel. Als jij op mijn 7e verjaardag niet mijn eerste dagboek gegeven had, was ik misschien wel nooit onderzoeker geworden! Wat ben ik blij met jou, als peettante, maar vooral als zeer dierbaar persoon in mijn leven die er altijd voor mij is en mij ook ècht goed kent. We hebben altijd veel contact gehad maar in de laatste jaren des te meer; ik waardeer het heel erg dat je altijd zo betrokken bent, zoveel lieve kaartjes stuurt en altijd positief bent. Ik zag en zie je nog steeds als groot voorbeeld en vind je onwijs stoer! Laten we nog lang samen de leukste dag van het jaar (onze verjaardag) vieren en elkaar blijven inspireren. Dankjewel lieve Miek!

Roger, we don't go way back. We kennen elkaar inmiddels 'pas' 3 jaar, wat jou een van de newbies in dit dankwoord maakt. Jouw relativerende woorden en relaxte houding heb ik altijd heel erg gewaardeerd. Ook het feit dat je niet vies was van een (felle) discussie vond ik erg fijn en jouw rake analyses over, tja, nagenoeg alles, hebben mij veel gebracht. Het inzicht dat je me gaf, een soort van socratische ' $k$ weet dat $i k$ niets weet', heb ik altijd in mijn achterhoofd zitten als ik het mezelf weer eens kwalijk neem dat ik te weinig kennis in huis heb. Ik denk dat ik een boekwerk minstens zo dik als vijf proefschriften aan elkaar kan rijgen op basis van onze ellenlange Whatsapp-conversaties. We blijven een bijzonder verhaal. Bedankt voor al je interesse en steunende woorden en voor onze vriendschap, ik hoop dat we elkaar nog heel veel gaan uitdagen en inspireren. En, blijf alsjeblieft altijd zo breed geïnteresseerd en knotsgek als je bent!

Tom, mijn oud(st)e vrind. Toen ik je 11 jaar geleden ontmoette op TP had je gelijk mijn aandacht, welke nooit is weggegaan. Destijds waren we nog halve pubers en in de loop der jaren hebben we allebei een mooie weg bewandeld naar het volwassen leven. Dit is ook goed merkbaar geweest in onze vriendschap; van samen naar concerten gaan en praten over ditjes en datjes, naar ellenlange (serieuze en minder serieuze) gesprekken over volwassen worden. Wat vind ik het fijn dat we elkaar nooit uit het oog verloren zijn en, met name de laatste drie jaar, zoveel voor elkaar betekend hebben. Ik moet altijd stiekem lachen als jij mij uitlegt hoe het in Limburg reilt en zeilt, terwijl ik inmiddels zelf zo'n zes jaar in het uiterste zuiden heb vertoefd. Desondanks kan ik nog steeds geen Limburgs, maar vind ik het altijd heerlijk om jou Remunjs te horen praten. Tom, veel dank voor je vriendschap en lieve woorden door de jaren heen en ik hoop dat we nog lang in elkaars leven blijven en dat ik nog veel van jouw muziek mag genieten! Caro, jij mag natuurlijk niet ontbreken in dit stukje. Waar Tom en ik from scratch al bevriend raakten, vonden wij elkaar pas meer richting het einde van TP. We bleken best veel op elkaar te lijken en gemeenschappelijk te hebben. Ook al woon je (nu nog wel) in München, we blijven elkaar vinden. Ik zou het natuurlijk leuk vinden als je weer 
terugkomt naar Nederland zodat we elkaar vaker kunnen zien, maar ik kom ook graag weer langs in Duitsland! Lieve Caro, bedankt dat je altijd zoveel interesse hebt gehad in mijn werk en dat je me - op afstand - altijd hebt gesteund en geïnspireerd!

Denise, ik vergeet nooit meer dat jij mij opbelde met de vraag of ik de oppas van Owen en Megan wilde zijn. Ik was nèt begonnen met mijn master en toen ik hoorde dat jij óók psycholoog was en zelfs gepromoveerd in Maastricht maakte mijn hart al een sprongetje - soort zoekt soort! Ik voelde me kortom vereerd om op jouw kinderen te gaan passen. Vanaf het begin was er niet alleen een klik tussen de kinderen en mij, maar ook tussen ons. Steeds langer bleef ik 'toevallig' hangen na het oppassen, waardoor Bob moest wachten met het kijken van zijn series. We raakten maar niet uitgepraat over psychologie en het doen van onderzoek. Toen jij mij aan jouw keukentafel hielp met mijn masterscriptie, had ik nooit gedacht noch verwacht ooit zelf te gaan promoveren. Maar toch, altijd als ik jou vol passie hoorde praten over de wetenschap ging het kriebelen. Ik kan dus met zekerheid zeggen dat jij één van mijn belangrijkste inspirators bent geweest, voor én tijdens mijn PhD. Lieve Denise, héél veel dank voor je interesse, het meedenken, het geven van fijne adviezen en jouw relativerende kijk op zaken. We hebben ons vaak afgevraagd 'wat we nou precies zijn van elkaar', maar ik reken je inmiddels tot vriendin hoor! Heel erg bedankt!

Claire, ik zal nooit het opbloeien van onze vriendschap bij HSK Breda vergeten. Ik keek ontzettend op tegen jou als toen bijna GZ-psycholoog - terwijl ik pas net kwam kijken als kersverse therapeut - en was dan ook vereerd dat jij samen met mij naar een bruiloft van onze collega wilde gaan. Heel gauw merkte ik hoe leuk wij klikten en dat onze gesprekken moeiteloos uuuuren konden duren. Inmiddels zijn we 6 jaar bevriend (we moeten nog steeds ons 5-jarig jubileum vieren!) en kijk ik terug op hele mooie, maar ook uitdagende periodes. Jouw vermogen om inzichtgevende vragen te stellen, je mildheid, je steun, je luisterend oor en de heerlijke avondjes waarin we niet meer bij komen van het lachen waardeerde ik altijd ontzettend. Ik hoop dat we nog vele ontnuchterende, go-with-the-flow, héérlijke en eloquente feauwteauw-momenten in een idyllische ambiance mogen beleven samen; niet-tan? Alle gekheid aan de kant; zoals Daniël al in zijn dankwoord schreef wil ook ik jou laten weten dat ik trots op je ben. Je bent een prachtig persoon met een hart van goud. Vergeet dat nooit! Bedankt voor al je interesse in mijn onderzoek en het uitspreken van waardering en bewondering, dat heeft me altijd heel erg goed gedaan.

Lieve Kay, ik hoefde je maar één appje te sturen met de vraag of je mij zou kunnen helpen om ouders te werven via kinderdagverblijven, en onze onderzoeksflyer stond de dag erna al op jullie website. Buiten dat dit echt typeert hoe jij bent, namelijk behulpzaam, vastberaden en vlot, gaf het mij ook aan hoe fijn onze vriendschap is en hoe blij ik met je ben. We kennen elkaar inmiddels al zo'n 11 jaar en hebben heel veel samen beleefd. We staan voor elkaar klaar wanneer dit nodig is en kunnen ook samen genieten van een avondje in de kroeg. Hopelijk kunnen we daar weer mee doorgaan als de lockdown(s) een beetje voorbij zijn. Ik kan niet wachten om weer een weekendje Utrecht te doen samen! Je bent echt een inspiratie voor mij; moeder van twee schatjes van kinderen, heel veel bezig met zelfontwikkeling en daarnaast je eigen baan waarvoor je je heel hard inzet. Je bent een prachtmens, en ik ben heel erg trots op je! Ik hoop dat we nog jaren samen kunnen genieten en dat ik nog héél veel mag meemaken met Len en Liv. Bedankt voor onze vriendschap en jouw steun!

Lotje (ik blijf je zo noemen hoor, ik geloof niet dat je het erg vindt), mijn allereerste maatje! Wij hebben werkelijk waar alles meegemaakt! Van in jullie boomhut klimmen en op ons klimrek hangen, tot spelen met poppen en skelteren door de bosjes op de Steenoven. En nu zijn we opeens ruim 25 jaar verder, allebei (bijna) 30 (help!), en verdiepen we onze vriendschap nog steeds. Ik waardeer onze band enorm en kan er echt van genieten dat wij nooit uitgepraat raken. We spreken dit al heel vaak naar elkaar uit, maar ik doe er hier gewoon nog even een schepje bovenop! Je bent altijd super geïnteresseerd, lief en betrokken en ik hoop dat we nog heel veel samen gaan beleven. Wat ik vooral zo fijn vind aan ons, is dat we het vaak juist niét over werk hoeven te hebben. We weten natuurlijk wel waar we beiden mee bezig zijn, maar zijn vooral geïnteresseerd in alles wat er buiten ons werk gebeurt. Ik vond het zo leuk dat je een poos geleden, met een glimlach op je gezicht, voorzichtig vroeg: 'Mag ik dan ook bij jouw verdediging zijn?'. Natuurlijk! Ik hoop dat de omstandigheden het toelaten, maar als er iémand bij hoort, ben jij het Lot! Bedankt voor al je lieve woorden, al je kaartjes en het uitspreken van je waardering in de afgelopen jaren - dat heeft me altijd heel 
Ellen \& Kelly. De drie musketiers van Stratumsedijk 103b! Wat is het leuk dat wij elkaar nog steeds, jaren nadat ons studentenhuisje zelfs al gesloopt is, weten te vinden. Ik ben heel blij met jullie en vind het erg fijn om vooral veel met jullie te lachen; we halen het gekste in elkaar naar boven. Hopelijk blijven we nog jaren herinneringen ophalen aan onze studententijd en nieuwe herinneringen maken; let the Ell-Karl-Kell saga continue! Bedankt voor jullie steun lieve ladies! Ell, jou noem ik nog even in het bijzonder. Alleen al om het feit dat jij mij Stijn geïntroduceerd hebt en ik daardoor een awesome designer op mijn pad kreeg om dit proefschrift samen mee vorm te geven. Maar, des te meer wil ik je bedanken voor alles wat je voor mij betekent. Jij bent een tough cookie (vooral voor jezelf) en gigantisch lief en zorgzaam tegelijkertijd. Ik maak graag gebruik van dat laatste (lees: als je weer lekker voor me kookt), maar kan ook veel hebben aan jouw nuchtere blik op zaken. Jij staat heel dicht bij jezelf en dat heb ik altijd erg mooi gevonden. Blijf alsjeblieft zo lekker jezelf, want dat maakt je echt uniek en bijzonder. Ik vind het heerlijk dat wij samen overal voor te porren zijn, wat leidt tot de meest gekke out-of-the-box gesprekken en activiteiten. Daarnaast kunnen wij elkaar als geen ander door the good, the bad and the ugly slepen, zoals we hebben laten zien in de afgelopen 10 jaar. Laten we dat altijd blijven doen. Ik kijk uit naar alle uitstapjes die we samen nog gaan maken, of dat nou boekenbeurzen afspeuren, festivals en feestjes afstruinen, stedentripjes doen, uiteten gaan of knus op de bank zitten met een kleedje is; met jou is alles leuk!

Eline. Zoals jij in jouw dankwoord omschreef, kennen onze levens veel parallellen; Elst, Eindhoven, op kamers, studeren, naar het buitenland, promoveren... Ondanks dat ik ons beiden over enige tijd nog steeds aan de andere kant(en) van de wereld zie vertoeven, houden de gelijkenissen daar wel even op. Voor nu althans. Want jij bent met Sam en Fosse een kersvers gezinsleven gestart en ik ben daar vooral heel erg van aan het meegenieten! We hebben ons samen zo vaak afgevraagd of we voor een carrière en hard werken zouden willen gaan, of meer wilden/moesten focussen op settling down. Ik denk dat jij als geen ander laat zien dat beiden heel goed hand in hand kunnen gaan. Wat heb ik altijd tegen je opgekeken; als slimme en ambitieuze vrouw in de (vooral) mannelijke wereld van de biomedische wetenschap. Je stond en staat altijd je mannetje, waarbij het soms net lijkt of het je zomaar komt aanwaaien. Maar ik weet en zie ook hoe hard je altijd werkt en hoe krachtig je bent! lk was dan ook ontzettend trots toen ik jou als paranimf mocht bijstaan tijdens jouw verdediging. Nu ben ik wederom onwijs trots op je als ik zie hoe 'natuurlijk' jij het moederschap op je neemt; wauw! Je bent echt een van de meest authentieke en inspirerende mensen die ik ken en met jou kan ik werkelijk over álles filosoferen. Maar, we kunnen het leven samen ook vaak juist niét zo serieus nemen, wat de boel altijd weer een beetje in perspectief plaatst. Jij liep steeds één jaar voor op mijn promotie, wat me erg heeft geholpen te relativeren - ook al schrok het me soms ook echt af. Het gesprek dat we voerden toen jij jouw discussie aan het schrijven was, over wat nou maakt of iemand zijn PhD afrondt of niet, gaf mij destijds echt de bevestiging om door te zetten. Het was (en is) heel fijn om met jou wederom ervaringen uit te wisselen in mijn laatste fase van het proefschrift en de verdediging. Ik kan niet wachten om jou straks in de zaal te zien stralen! Lieve Eline, wat ben ik dankbaar voor onze vriendschap en voor de inspiratie die we voor elkaar zijn! Bedankt voor alles en op naar nog veel meer jaren - met lieve Fosse erbij!

Lieve Jill, terwijl ik dit schrijf zijn wij al ruim 20 jaar vriendinnen; in de bovenbouw van 't Startblok ontstond onze band en deze is gelukkig nooit meer weggegaan. Ik verruilde Elst al snel voor Eindhoven en jij verhuisde een paar jaar later naar Nijmegen. Ondanks dat de fysieke afstand groter werd, gebeurde met onze vriendschap het tegendeel. Het was geen toeval dat wij beiden kozen voor een sociale studie; ik denk dat onze interesse in gedrag en de behoefte om mensen te begrijpen en verder te helpen namelijk één van de redenen is waarom onze vriendschap zo sterk is. Wij zijn elkaars grootste fan en wat vinden we altijd veel herkenning bij elkaar! Ik ken niemand die zo loyaal, oprecht, enthousiast en zorgzaam is als jij. Ik weet altijd wat ik aan je heb en hoef me nooit anders voor te doen dan ik ben, en dat is me erg dierbaar. Als wij samen zijn, halen wij echt het allerbeste bij elkaar naar boven. Ik ben ontzettend trots op je en ben heel dankbaar dat ik bij elke belangrijke stap in jouw leven heb mogen zijn, en vice versa. Zoals we inmiddels zó vaak op (al die 1000) kaartjes hebben geschreven: we staan elkaar, wat er ook gebeurt, op te wachten aan de finish! En ja, jij bent er straks tijdens mijn verdediging wederom bij - op de allereerste rij. Ik wil je ontzettend bedanken voor de steun, de vrolijke noot en de onuitputtelijke bron van vertrouwen die je altijd voor me bent. Als ik het allemaal even niet meer zag zitten, was jij daar altijd om me aan te moedigen, je vertrouwen in mij uit te 
spreken, en vooral ook om te erkennen dat het soms gewoon even minder mag gaan. Dankjewel voor zoveel lieve Jill, en op naar alles wat ons nog te wachten staat samen!

Suus, lieve 'zus' en paranimf. Samen gaan wij al ruim tien jaar het avontuur 'des levens' aan en wat hébben we veel beleefd. Toen ik je de eerste keer ontmoette vond ik je al meteen interessant; je was zo spontaan, geïnteresseerd en authentiek. Al snel werden we bevriend en studeerden we samen in Eindhoven, Tilburg en Maastricht. Wat kijk ik vooral bijzonder terug op onze Mental Health tijd; alle ritjes die we samen (met sloten koffies) maakten naar Maastricht waren zó gezellig! We waren ook elkaars vaste uitlaatklep; we begrepen elkaar precies en sleepten elkaar door alle hectiek van studeren, werken en klinische stages heen. Het zijn van elkaars maatje is na onze studententijd (gelukkig) nooit meer weggegaan. Allesbehalve; we zijn alleen maar dichter naar elkaar toe gegroeid. We vogel(d)en samen uit wie we zijn en wat we waarderen in het leven, we reizen of kamperen, we sporten samen, we zwijmelen samen weg bij de leukste inwoner van Eindhoven (THEO), we kunnen uren filosoferen en (psychologisch) analyseren en ga zo maar door. Lieve zus, er is niks wat ik met jou niet kan of kon doen en dus ook niks wat ik zonder jou niet aan durf of zal doen. Ik kan me dan ook niemand anders wensen die achter mij zal staan tijdens mijn verdediging. Je bent echt mijn steun en toeverlaat geweest in de afgelopen jaren. Ik vond het heel fijn dat jij zo oprecht geïnteresseerd bent in mijn werk en de studies die ik deed en me keer op keer bleef zeggen hoe knap je het vond. Als ik het ook maar waagde om mijn werk af te doen als iets onbelangrijks of waar ik niet met trots over zou mogen vertellen, stimuleerde jij mij om wat minder bescheiden te zijn. Het feit dat jij mij - en waarschijnlijk als de enige persoon ever - als 'Thoonen et al.' in je telefoon hebt staan, says it all! Jouw relativeringsvermogen is echt goud waard en daar heb ik dan ook heel vaak (onbewust) een beroep op gedaan. Je bent een prachtvrouw die heel bijzonder in haar leven staat. Ik ben ontzettend trots op je en hoop dat je altijd de mooie, oprechte en zorgzame persoon mag blijven die je bent. Ik zal je op mijn beurt altijd blijven helpen de 'spinnen' uit je leven weg te halen. Laten we na vandaag nog jaren vol ervaringen beleven; let's jump together!

Lieve Esther, ik ga niet voor de zoveelste keer ons 'How we've met' verhaal herhalen. Of kom ik er eigenlijk niet onderuit? Vooruit dan maar ... op 1 oktober 2016, de dag dat we in kantoortje B1.112 verwelkomd werden met twee plantjes voor de deur, begon onze gezamenlijke promotie-rollercoaster. Beiden nog géén idee wat ons te wachten stond en wat er tussen ons zou ontstaan. Met onwennige vragen als 'Goh ja, waar kom jij vandaan dan?' en 'Weet jij eigenlijk wat je allemaal gaat doen?' probeerden we wat aan elkaar te wennen, maar we dachten er beiden het onze van. Gelúkkig kwam daar de dag dat we besloten onze bureaus om te draaien en niet langer met de rug naar elkaar te zitten; vanaf toen konden we geen genoeg meer van elkaar krijgen. We bleken al snel een supermatch en onze lach begon tot behoorlijk ver in de GVO-gang te reiken (sorry collega's). We kwamen al gauw op dat punt dat we aan één blik genoeg hadden om te weten hoe de vlag erbij hing bij de ander. Wat wil je ook, als je dag in, dag uit, 8 uur per dag aan elkaar gebonden bent in een kantoortje van $12 \mathrm{~m}^{2}$. We waren gewaagd aan elkaar; we vulden elkaar aan en konden elkaar sterk motiveren. We zijn in sommige opzichten echte tegenpolen; jij wat directer, ik wat meer voorzichtig; jij planmatig, ik wat meer go with the flow; jij de verteller en ik de vragensteller; jij de kokkin en ik de 'alles wat lekker is kan gewoon bij elkaar toch?'; jij de docent, ik de begeleider. Oh, oh, oh (eauw eauw eauw) wat waren - en zijn - we een stel samen. Es en Karrol. Kar en Estur. Ik heb de afgelopen jaren zoveel gehad aan jouw eerlijkheid en doortastendheid. Ik weet bij jou altijd wat ik aan je heb omdat je recht vanuit je hart spreekt, en dat is echt een gouden eigenschap. Onze lachbuien, de dagelijkse afwassessies waarin we de dag nog even nabespraken - en waar we overduidelijk een andere manier voor hadden, ik die hierdoor áltijd moest rennen voor mijn trein; jij die me dan altijd uitlachte of zei: 'Ja Kar, gá nou!' (terwijl jij vaak nog aan het kletsen bleef), onze verkleedpartij voor Rik's feest, ons jaarlijkse chocomelletje op het Vrijthof om de kerstvakantie in te luiden, jij die altijd op me wachtte met de 'loens' (zelfs als je al 3 uur lang trek had en ik weer eens ergens stond te kletsen), onze heerlijke koffietjes bij 'Adjuh' en later bij Rivazza (en waar het kletsen uiteraard non-stop doorging), de make-overs in ons kantoor als we weer even waren uitgekeken op de plantjes, kunstwerken of foto's aan de muur, onze muizeninvasie in het plafond, onze mega moestuinencollectie waar menig collega jaloers op was (vooral op die komkommerplant waar nooit komkommers uit gekomen zijn), tranen als we weer een lief of opbeurend kaartje voor elkaar geschreven hadden, ons potje met lekkers (die ik nooit had moeten meenemen naar werk), ik die aan het bellen was terwijl ik door het raam zag dat jij in de lach schoot om iets wat gezegd werd, onze Geer \& Goor middagen, jij die me weer eens hard lachend betrapte op een verkeerd gezegde - ondanks dat 'je ei luchten' inmiddels 
ook in jouw vocabulaire thuishoort, het meezingen op Marceauw Borsateauw, samen dromen over ons leven na het promoveren.... De lijst wordt echt te lang, zelfs voor mij, maar ik kan nog pagina's doorgaan met al onze ervaringen die onze PhD tijd uniek, fijn of gezellig hebben gemaakt. Het was me allemaal wat. Wat ben ik blij dat ik mijn PhD met JOU heb mogen beleven lieve Es. En wat super dat je naast en bij me staat als paranimf tijdens mijn verdediging. Ik had niets liever kunnen wensen. Bedankt voor ALLES en nog heel veel meer. Veel liefs, Karthelijne Thole.

Voor de lezer die het tot hier volgehouden heeft: Chapeaux! Ik heb zelf lange tijd het gevoel gehad dat ik mijn PhD aan het 'volhouden' was. Zoals de gemiddelde promovendus ben ook ik mezelf meermaals tegen gekomen, heb ik getwijfeld over mijn kunnen maar heb ik een gigantische dosis zelf- en professionele kennis opgedaan. Ik ben heel erg blij dat mijn gevoel van volhouden door de jaren heen omsloeg in enthousiasme, positieve energie en vertrouwen. Vertrouwen in vooral mijzelf; ik had lang het idee dat het mij niet zou gaan lukken, maar gaandeweg kon ik die overtuiging loslaten. Zolang ik maar in mezelf zou geloven, zou alles wel goed gaan komen. En dat gebeurde ook. Na mezelf vooral vaak tegen te komen, heb ik mijzelf eindelijk eens ontmoet. Het zijn bewogen jaren geweest, maar ik had deze reis voor geen goud willen missen. Het feit dat het laatste, tevens meest spannende en leukste (want: schrijven!) gedeelte van mijn proefschrift nagenoeg volledig thuis afgerond moest worden wegens COVID-19 maakte de afronding van mijn 'PhD-experience' nog eens extra uitdagend maar zeker ook bijzonder. De laatste loodjes zijn vaak het zwaarst, maar ik vond ze ook juist het allerleukst. En wat ben ik trots op het eindresultaat! Dankzij velen van jullie in dit laatste hoofdstuk heb ik inspiratie opgedaan, ontzettend kunnen groeien, leren te geloven in mijn kunnen en mijzelf leren zien zoals jullie mij zien. Ik ben jullie allemaal zeer dankbaar!

\section{'I need to laugh, and when the sun is out, I've got something I can laugh about'}

Good day sunshine - The Beatles 

'No borders, just horizons - only freedom.' 